\title{
Regulation Of Spindle Orientation By A Mitotic Actin Pathway In Chromosomally Unstable Cancer Cells
}

\author{
Dissertation \\ for the award of the degree \\ "Doctor rerum naturalium" \\ Of the Georg-August-Universität Göttingen \\ within the doctoral program "Molecular Medicine" \\ of the Georg-August University School of Science (GAUSS) \\ submitted by \\ Nadine Schermuly
}

from Weilburg an der Lahn, Germany

Göttingen, September 2019 


\section{Thesis Committee}

Prof. Dr. Holger Bastians

Institute for Molecular Oncology

Section of Cellular Oncology

University Medical Center Göttingen

Prof. Dr. Jörg Großhans

Center for Biochemistry and Molecular Cell Biology

Section Developmental Biochemistry

University Medical Center Göttingen

Prof. Dr. Michael Zeisberg

Department of Nephrology and Rheumatology

University Medical Center Göttingen

\section{Members of the Examination Board}

Referee: $\quad$ Prof. Dr. Holger Bastians

Institute for Molecular Oncology

Section of Cellular Oncology

University Medical Center Göttingen

$2^{\text {nd }}$ Referee: Prof. Dr. Jörg Großhans

Centre for Biochemistry and Molecular Cell Biology

Section Developmental Biochemistry

University Medical Center Göttingen

\section{Further Members of the Examination Board}

Prof. Dr. Michael Zeisberg

Department of Nephrology and Rheumatology

University Medical Center Göttingen

Prof. Dr. Matthias Dobbelstein

Institute for Molecular Oncology

University Medical Center Göttingen

Prof. Dr. Andreas Janshoff

Institute of Physical Chemistry

University of Göttingen

Prof. Dr. Dieter Kube

Department of Haematology and Oncology

University Medical Center Göttingen

\section{Date of Oral Examination}




\section{Affidavit}

Hereby I declare that my doctoral thesis entitled "Regulation Of Spindle Orientation By A Mitotic Actin Pathway In Chromosomally Unstable Cancer Cells" has been written independently with no other sources and aids than quoted.

Göttingen, September 2019

Nadine Schermuly 


\section{Table Of Contents}

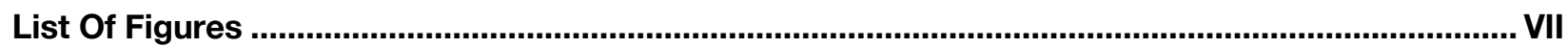

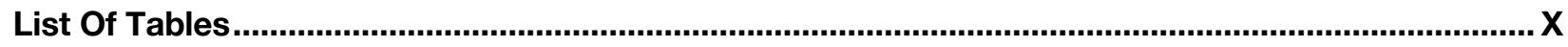

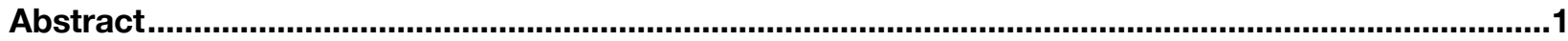

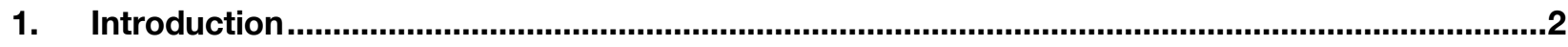

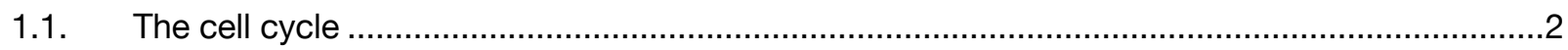

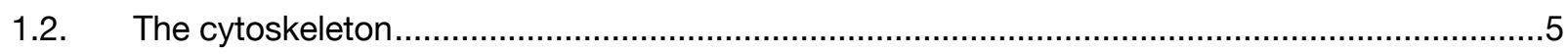

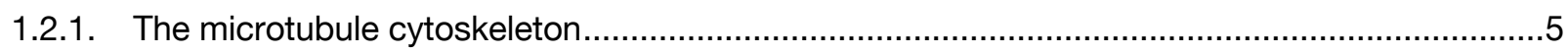

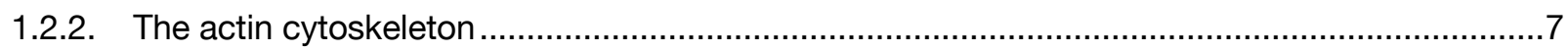

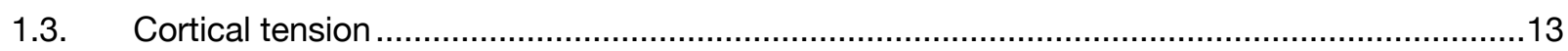

1.4. Crosstalk between microtubule and actin cytoskeleton ..................................................15

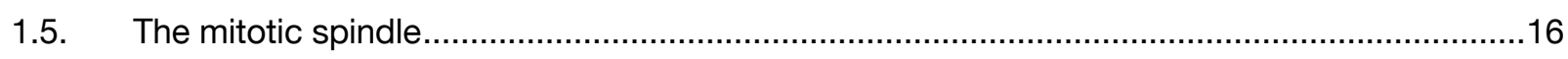

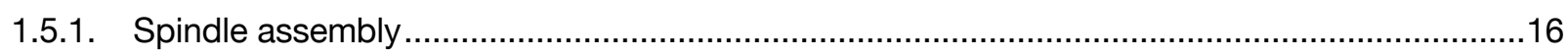

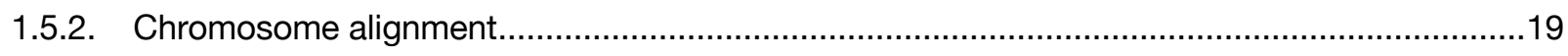

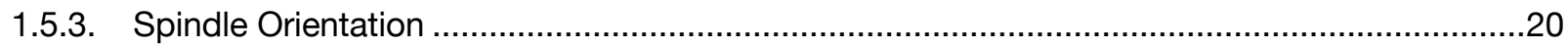

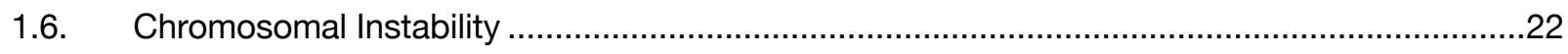

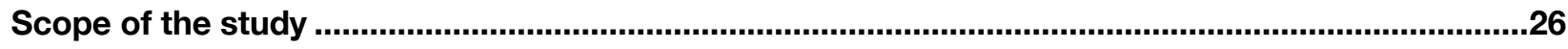

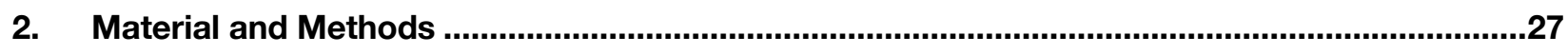

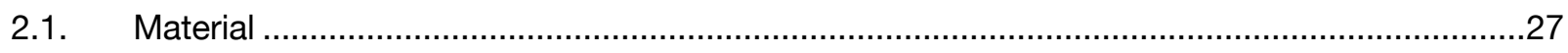

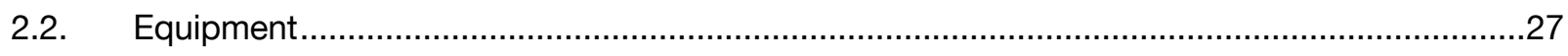

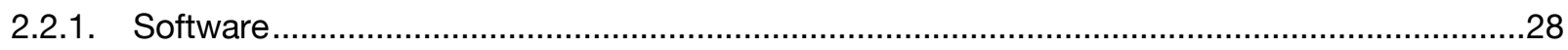

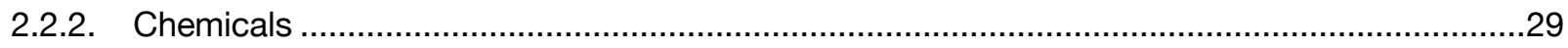

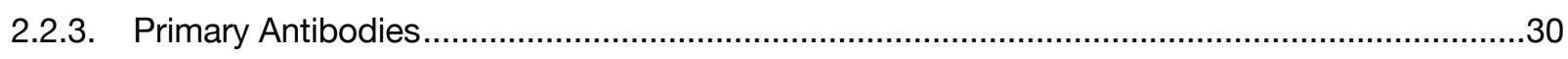

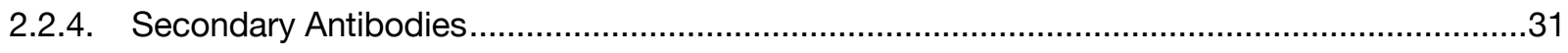

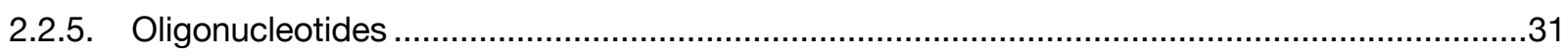

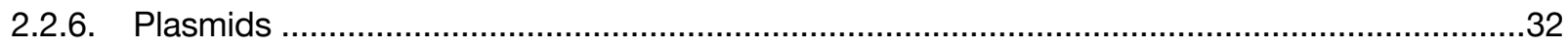




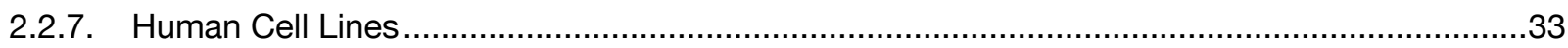

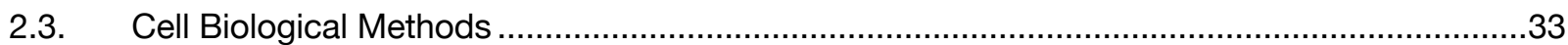

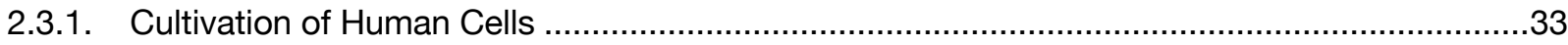

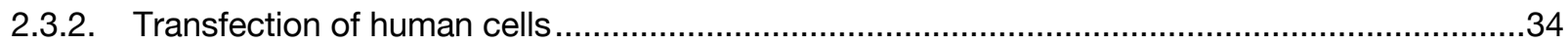

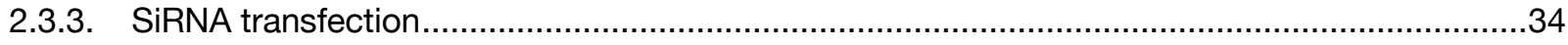

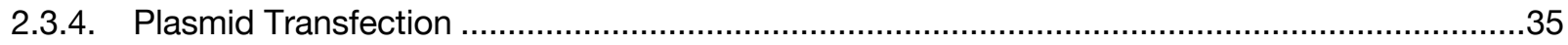

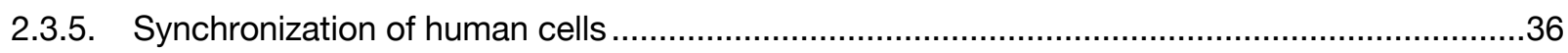

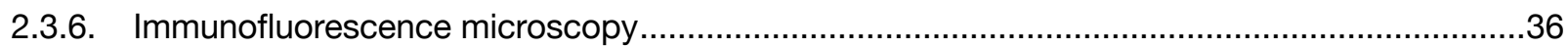

2.3.7. Analysis of spindle axis alignment in prophase, prometa- and metaphase ...........................37

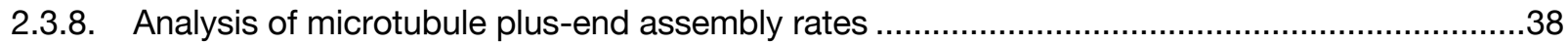

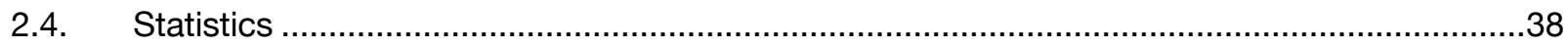

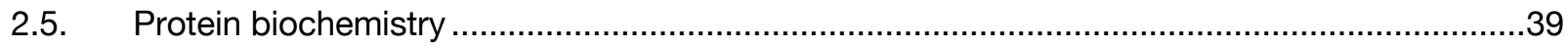

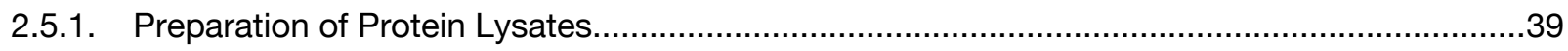

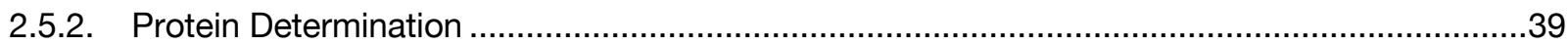

2.5.3. Sodium Dodecylsulfate Polyacrylamide Gel Electrophoresis (SDS-PAGE) .............................39

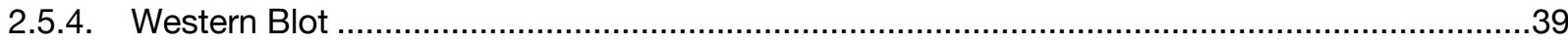

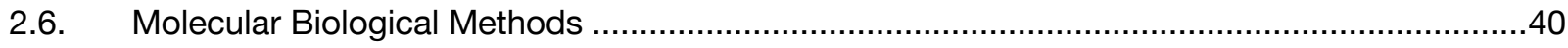

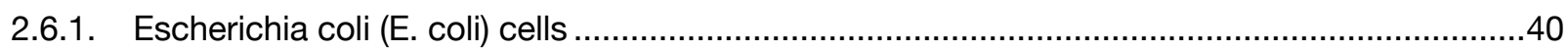

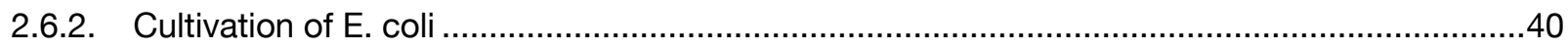

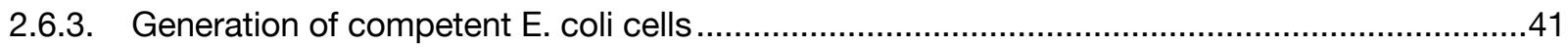

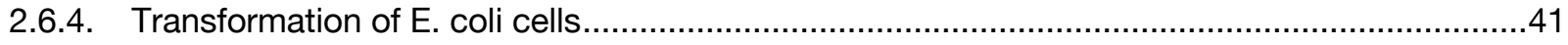

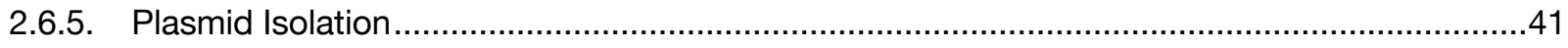

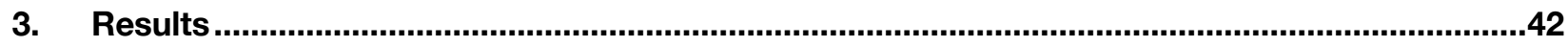

3.1. Abnormal spindle structures of CIN cells are associated with a transient spindle axis

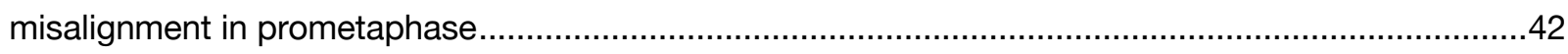

3.2. Enhanced microtubule plus-end assembly rates cause spindle axis misalignment in prometaphase. 
3.3. Spindle axis misalignment promotes chromosome mis-segregation in chromosomally stable HCT116 cells

3.4. Deregulation of the actin cortex cytoskeleton leads to spindle axis misalignment in prometaphase and promotes the generation of lagging chromosomes.

3.5. Decreased cortical tension during mitosis is responsible for spindle axis misalignment in prometaphase.

3.6. The Rac1-Arp2/3 pathway plays a role for spindle axis alignment exclusively in chromosomally unstable cells.

3.7. A Rac1-Arp2/3 pathway is responsible for spindle axis misalignment in chromosomally unstable cells downstream of microtubule plus-end assembly rates

3.8. TRIO - a link between the microtubule and actin cytoskeleton that mediates spindle axis misalignment in chromosomally unstable cells with enhanced microtubule plus-end assembly rates .69

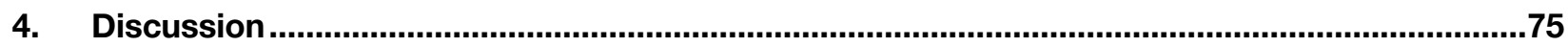

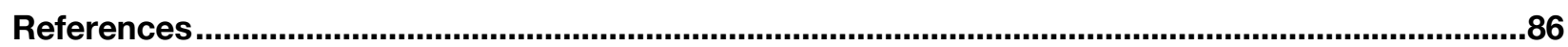

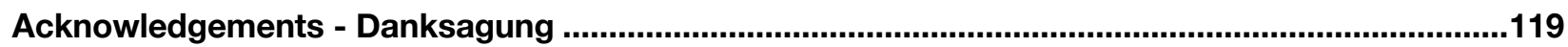

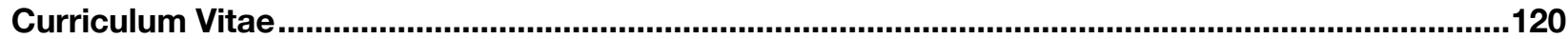




\section{List Of Figures}

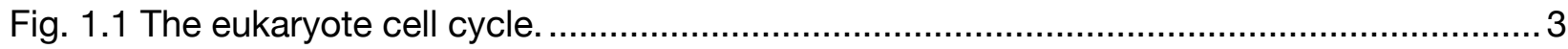

Fig. 1.2 Regulation of cell cycle progression by Cdks and Cyclins ...................................... 4

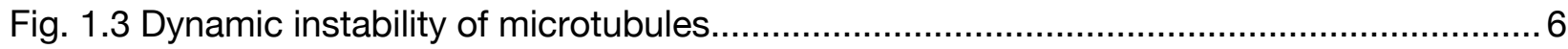

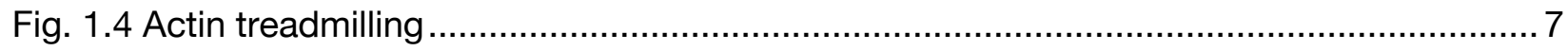

Fig. 2.1 Illustration for calculating the angle of spindle axis alignment................................... 38

Fig. 3.1 CIN cells exhibit a higher proportion of cells with lagging chromosomes in anaphase. 42

Fig. 3.2 Altered spindle structures of CIN cells correlate with an increased angle of spindle axis alignment in prometaphase.

Fig. 3.3 Spindle axis misalignment in CIN cells is present prometaphase but not in prophase and metaphase.

Fig. 3.4 Partial depletion of CKAP5, STATHMIN or low doses of Taxol have no effect on microtubule plus-end assembly rates, spindle axis alignment and lagging chromosomes in chromosomally stable HCT116 cells.

Fig. 3.5 Restoration of proper microtubule dynamics in CIN cells leads to proper spindle axis alignment in prometaphase and decreases the amount of lagging chromosomes in anaphase.

Fig. 3.6 Induction of enhanced microtubule plus-end assembly rates induces spindle axis misalignment and an increased proportion of cells with lagging chromosomes in chromosomally stable HCT116 cells. 50

Fig. 3.7 Induction of spindle axis misalignment by LGN knockdown induces lagging chromosomes.

Fig. 3.8 Interfering with the spindle tethering machinery induces spindle axis misalignment and lagging chromosomes.

Fig. 3.9 Pertussis toxin-induced spindle axis misalignment in prometaphase causes whole chromosome mis-segregation in HCT116 cells.

Fig. 3.10 Modulation of actin polymerization affects spindle axis alignment in prometaphase and chromosome segregation. 
Fig. 3.11 Actin polymerization is mediated by increased microtubule plus-end assembly rates to regulate spindle axis alignment and chromosome segregation.

Fig. 3.12 Inhibition of myosin II induces spindle axis misalignment and lagging chromosomes in HCT116 cells and restores proper spindle axis alignment and chromosome segregation in CIN cells.

Fig. 3.13 Inhibition of ROCK restores proper spindle axis alignment and chromosome segregation in CIN cells and induces spindle axis misalignment and lagging chromosomes in HCT116 cells.

Fig. 3.14 Restoring proper microtubule plus-end assembly rates increases cortical tension $T_{0}$ of mitotic SW620 cells.

Fig. 3.15 In contrast to inhibition of the Rac1-Arp2/3 pathway, suppression of RhoA signaling induces spindle axis misalignment and the generation of lagging chromosomes in chromosomally stable HCT116 cells.

Fig. 3.16 Inhibition of Rho signaling and repression of the Rac1-Arp2/3 pathway restores proper spindle axis alignment in prometaphase and chromosome segregation in chromosomally unstable cells. 62

Fig. 3.17 DIAPH1 and the Rac-Arp2/3 pathway have no influence on microtubule plus-end assembly rates.

Fig. 3.18 Formin inhibition restores proper spindle axis alignment and chromosome segregation in CIN cells whereas it promotes chromosomes mis-segregation and spindle axis misalignment in MIN/MSI cells.

Fig. 3.19 RhoA activation leads to spindle axis misalignment and chromosome mis-segregation in chromosomally stable HCT116 cells but has no effect on CIN cells.

Fig. 3.20 Spindle axis misalignment induced by RhoA activation is only restored by formin inhibition but not by Arp2/3 inhibition.

Fig. 3.21 The Rac1-Arp2/3 pathway is responsible for spindle axis misalignment and chromosome mis-segregation downstream of microtubule plus-end dynamics. 68

Fig. 4.1 Model illustrating the role of an unscheduled TRIO-Rac1-Arp2/3 pathway for spindle axis misalignment and the development of CIN. 76

Fig. 4.2 Summary of treatments influencing cortical tension and spindle orientation. 78 
Fig. 4.3 Outcome of interfering with actin dynamics on spindle axis alignment and cortical tension. . .79

Fig. 4.4 Model of how actin cortex architecture influences cortical tension. 81

Fig. 4.5 Models to reduce cortical tension by enhanced microtubule plus-end assembly rates during mitosis. 


\section{List Of Tables}

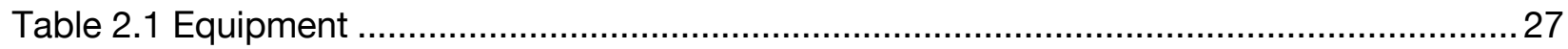

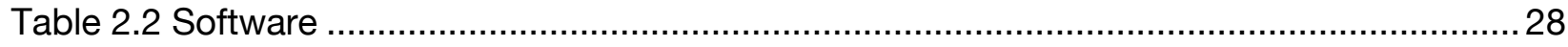

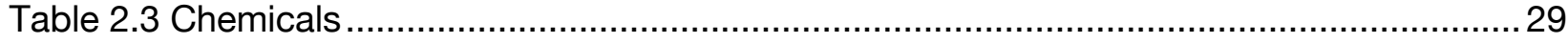

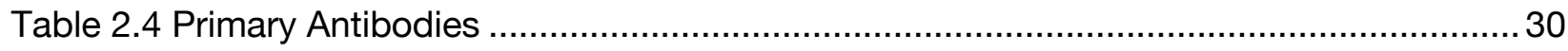

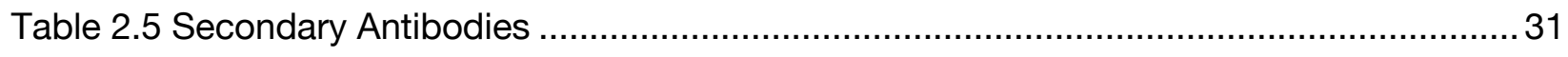

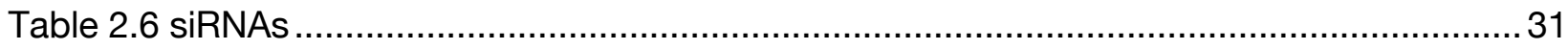

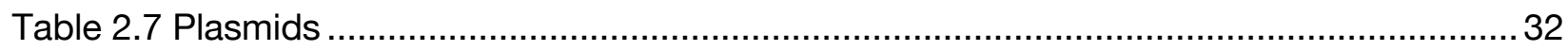

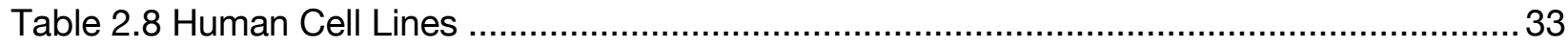




\section{Abstract}

Aneuploidy is a hallmark of human cancer and is observed in $90 \%$ of solid tumors. The perpetual gain or loss of whole chromosomes, defined as chromosomal instability (CIN), represent one mechanism causing aneuploidy. CIN cells are characterized by genetic heterogeneity, which allows the development of therapy resistance and promotes tumorigenesis. Thus, poor prognosis and patient's outcome is associated with CIN. Recently, it was revealed that CIN cells exhibit increased microtubule plus-end assembly rates during mitosis, which lead to spindle geometry defects, thereby facilitating the generation of lagging chromosomes and finally causing CIN. Moreover, first results revealed a role of a hyperactive TRIO-Rac1-Arp2/3 pathway mediated by increased microtubule dynamics during mitosis, which promotes spindle misorientation. Thus, the purpose of this study was to further analyze the role of a TRIO-Rac1-Arp2/3 pathway and how it causes spindle misorientation in CIN cells.

The present study revealed that CIN cells are characterized by a transient spindle axis misalignment during prometaphase, caused by enhanced microtubule plus-end assembly rates in mitosis, which finally leads to chromosome mis-segregation and CIN. The pathway causing spindle axis misalignment and chromosome mis-segregation in CIN cells was already identified previously (Berger, 2016). However, this work revealed that a mitotic EB1 dependent TRIO-Rac1Arp2/3 pathway in response to enhanced microtubule plus end assembly rates leads to increased actin polymerization, which reduces cortical tension in mitotic CIN cells. Moreover, the present study indicates that a RhoA-formin pathway leading to unbranched actin polymerization ensures proper cortical tension, and thus accurate spindle axis alignment in prometaphase. This pathway is active in both, chromosomally stable and unstable cells whereby in CIN cells, due to enhanced microtubule plus-end assembly rates, a TRIO-Rac1-Arp2/3 pathway deregulates mitotic actin cortex structures, which impairs the generation of cortex tension required for proper spindle axis alignment in prometaphase. Thus, these results demonstrate that a microtubule triggered actin pathway during mitosis causes spindle axis misalignment and chromosome mis-segregation in CIN cells. 


\section{Introduction}

\subsection{The cell cycle}

The eukaryotic cell cycle ensures generation of two daughter cells which equal the parental cell and comprises interphase and mitosis. The former is subdivided into three phases namely, $\mathrm{G}_{1}$ phase (Gap 1 -phase), S-phase (DNA synthesis phase) and $\mathrm{G}_{2}$-phase. Mitosis includes prophase, prometaphase, metaphase, anaphase and telophase (Nigg, 2001) (Fig. 1.1).

During $\mathrm{G}_{1}$-phase the cell prepares for the following S-phase. In case of missing mitogenic signals and growth factors the cell exits the cell cycle and enters the $\mathrm{G}_{0}$-phase and stops to proliferate (Norbury \& Nurse, 1992; Yao, 2014). However, upon mitogenic stimuli, $\mathrm{G}_{0}$ cells can re-enter the cell cycle and after reaching the so-called restriction point, cells progress through the cell cycle independent of mitogenic stimuli (Bertoli et al., 2013; Yao, 2014). In S-phase the centrosome is duplicated, DNA is replicated and the resulting sister chromatids are connected by the cohesion complex (Laskey et al., 1989; Peters et al., 2008; Urbani \& Stearns, 1999). During $\mathrm{G}_{2}$, the cell prepares for subsequent mitosis.

Chromosome condensation takes place in prophase (Antonin \& Neumann, 2016) while centrosomes start to migrate along the nuclear envelope and exhibit increased microtubule nucleation to establish a bipolar mitotic spindle (Tanenbaum \& Medema, 2010). Breakdown of the nuclear envelope (NEBD) occurs during prometaphase. Furthermore, proteins at the centromere are assembled to form kinetochores, which can be captured by microtubules (Cheeseman \& Desai, 2008). As a result of microtubule-kinetochore attachments, chromosomes are aligned at the metaphase plate and each sister chromatid is bound to microtubules emanating from opposite poles (Cheeseman, 2014). Upon proper chromosome alignment, the anaphase-promoting complex (APC) leads to activation of separase, which cleaves a subunit of the cohesion complex thereby promoting segregation of sister chromatids (Peters, 2002). Chromosome movement towards spindle poles is based on microtubule depolymerization during anaphase $A$ whereas in anaphase B the spindle elongates (Scholey et al., 2003). Moreover, the position of the anaphase spindle defines the division plane and a cleavage furrow forms, which involves an actomyosin based contractile ring. During telophase, contraction leads to membrane ingression and the nuclear envelope is re-established around decondensed chromosomes (Glotzer, 2005; Walczak et al., 2010). Subsequently, cytokinesis results in complete separation of daughter cells (Glotzer, 1997). 


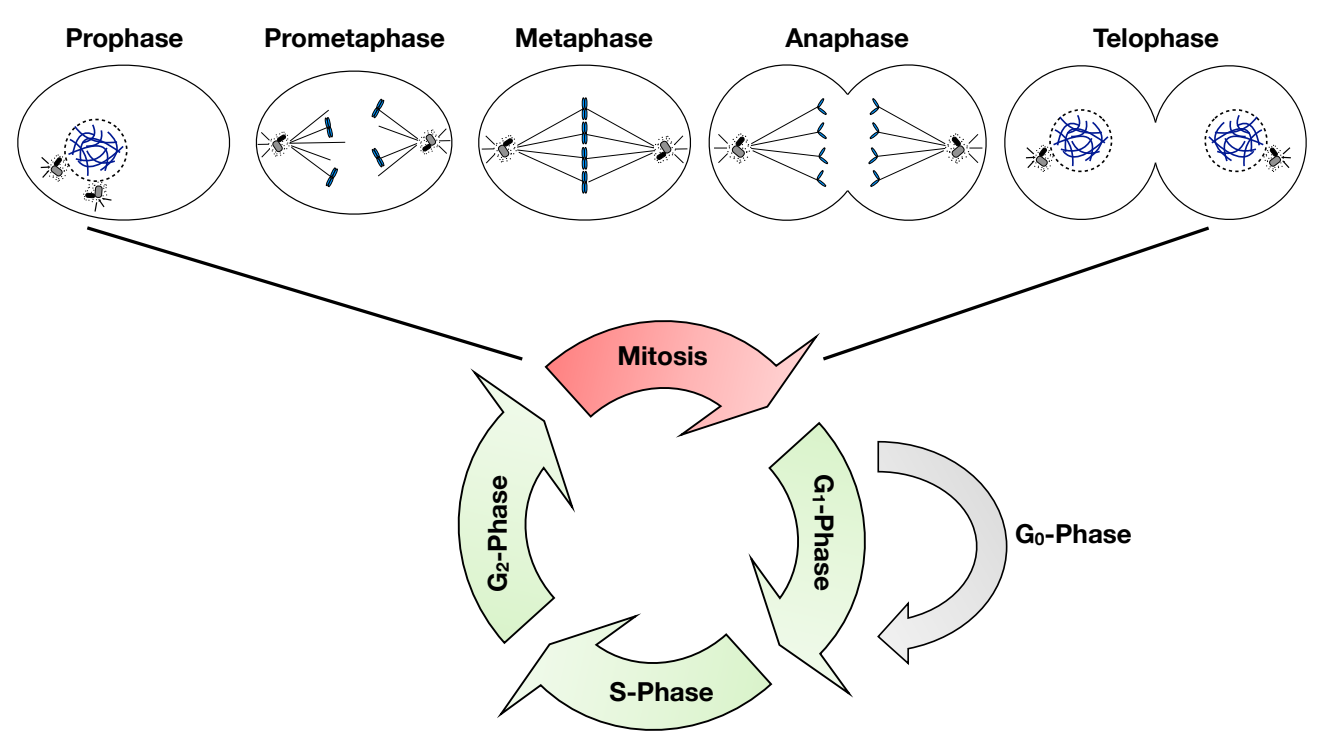

Fig. 1.1 The eukaryote cell cycle.

The cell cycle involves mitosis and interphase. The latter is divided into $\mathrm{G}_{1}$-phase, S-phase and $\mathrm{G}_{2}$-phase. Mitosis includes prophase, prometa- and metaphase, anaphase and telophase. During prophase the nuclear envelope breaks down and bipolar spindle assembly is initiated. Following bipolar spindle assembly and successful chromosome alignment at the metaphase plate, anaphase is initiated, and chromosomes are segregated into daughter cells. Subsequently, chromosomes decondense the nuclear envelope is reestablished.

Cell cycle progression is tightly regulated by cyclin-dependent protein kinases (Cdks), which are active when bound to their regulatory subunit called cyclins (Nigg, 1995). During cell cycle progression cyclins are regulated by well-timed synthesis and degradation (Malumbres \& Barbacid, 2009). In order to achieve full activation of Cdks, binding of Cdk-activating kinase (CAK) is required whereby $\mathrm{Cdk}$ inhibitory subunits $(\mathrm{CKI})$ are able to bind to CDKs or Cdk/cyclin complexes thereby blocking catalytic activity of Cdks (Morgan, 1997). Moreover, phosphorylation of Cdk-cyclin complexes by the kinases Wee1 or Myt1 leads to inactivation (Booher et al. 1997; Heald et al. 1993) whereas the phosphatase cdc25 (cell division cycle protein) reverses this effect (Morgan, 1997). Active Cdk/cyclin complexes influence transcription, translation and DNA replication as well as checkpoints during mitosis (Sánchez \& Dynlacht, 2005). During $\mathrm{G}_{1}$, Cdk4 and Cdk6 bind to D-type cyclins which leads to phosphorylation of retinoblastoma proteins ( $\mathrm{Rb}$ proteins) thereby preventing transcriptional repression of genes required for S-phase, such as Etype cyclins. As a result, cyclin $E$ binds to and activates $C d k 2$, which triggers $\mathrm{G}_{1}-\mathrm{S}$-phase transition (Malumbres \& Barbacid, 2005). During S-phase Cdk2 is no longer associated with cyclin E. Instead Cdk2 is activated by cyclin A, which is required for successful completion of S-phase and transition to $\mathrm{G}_{2}$. (Malumbres \& Barbacid, 2009). Additionally, in late S-phase Cdk1 in complex with cyclin A promotes transition from $\mathrm{G}_{2}$ to mitotic entry (Malumbres \& Barbacid, 2005). Subsequently, in $G_{2}$, cyclin $A$ is degraded and Cdk1 is activated by Cyclin B, which phosphorylates several 
targets, among them proteins that are involved in centrosome separation, chromosomal condensation and spindle assembly thereby leading to mitotic progression (Nigg, 2001). Degradation of B-type cyclins and subsequent inactivation of Cdk1 promotes mitotic exit (Malumbres \& Barbacid, 2005). Deregulation and overexpression of cyclins and cdks is often observed in human cancer (Malumbres \& Barbacid, 2001; Musgrove, 2006). Especially in chromosomally unstable tumors, genes involved in cell cycle regulation, chromosome segregation, centrosome integrity and spindle assembly are frequently overexpressed (Carter et al., 2006).

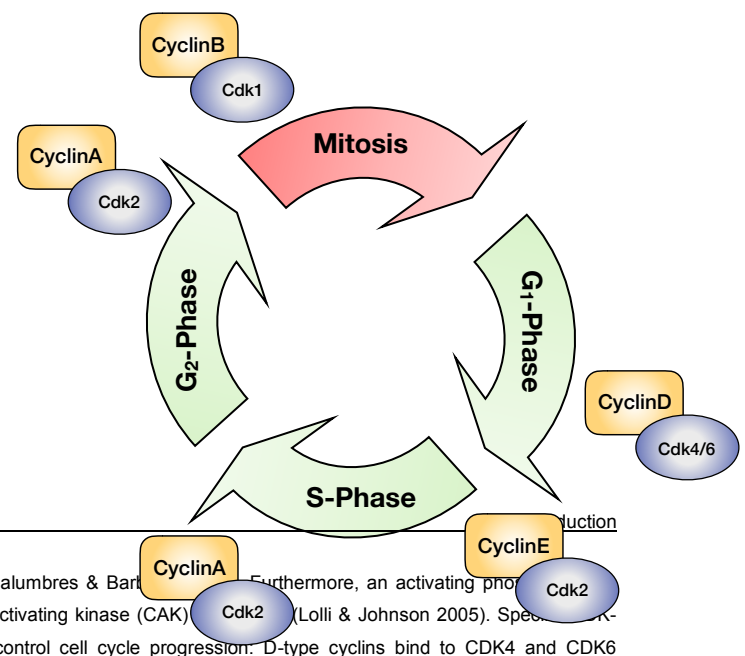

Fig. 1.2 Regulation of cell cycle progression by Cdks and Cyclins

Cyclin $D$ in complex with Cdk4/6 promotes transition from $\mathrm{G}_{1}$ into S-phase and promotes Cdk2 activation by Cyclin E, which triggers DNA replication. Subsequently, Cyclin A binds to Cdk2 for proper S-phase. In late $\mathrm{G}_{2}$, Cyclin A in complex with Cdk2 promotes entry into mitosis. Active Cdk1 bound to Cyclin B promotes mitotic progression.

Additional mechanisms, which are responsible to ensure proper cell cycle progression involve the DNA damage checkpoint and the spindle assembly checkpoint (SAC) (Nigg, 2001). In case of DNA damage or unreplicated DNA, the DNA damage checkpoint leads to cell cycle arrest and transcription of genes responsible for DNA repair and apoptosis (Zhou \& Elledge, 2000). The SAC ensures genomic integrity by monitoring microtubule-kinetochore attachments. If the SAC recognizes erroneous attachments, it delays transition to anaphase until chromosomes are bioriented and exhibit correct attachments (Musacchio, 2015). However, the SAC is not able to sense a specific type of mis-attachment, namely merotelic. These attachments are corrected by another mechanism involving Aurora B kinase at the kinetochore, which promotes microtubule turnover thereby facilitating correction of merotelic attachments (Cimini, 2007). 


\subsection{The cytoskeleton}

\subsubsection{The microtubule cytoskeleton}

In interphase, microtubules serve as tracks for transport of cargos and regulate cell shape (Goode, Drubin, \& Barnes, 2000; Tomasek \& Hay, 1984). Moreover, they play an important role for cell migration (Etienne-Manneville, 2013). In mitosis, microtubules are assembled into the mitotic spindle, which executes chromosome segregation (Wittmann et al., 2001).

Microtubules arise from polymerization of $\alpha$-tubulin and $\beta$-tubulin heterodimers, which are assembled in 13 protofilaments that form hollow tubes (Ohi \& Zanic, 2016). Those filaments exhibit a plus- and a minus-end, whereby $\alpha$-tubulin is present at the minus-end and $\beta$-tubulin is exposed at plus-ends. (Akhmanova \& Steinmetz, 2015). During polymerization, GTP bound $\beta$-tubulin is hydrolyzed. In case of delayed GTP hydrolysis, a structure called GTP cap occurs, which is more stable and promotes further polymerization whereas GDP bound $\beta$-tubulin is unstable and leads to depolymerization of microtubule plus-ends (Dimitrov et al., 2008). The constant process of adding tubulin heterodimers at plus-ends and dissociation of tubulin at minus-ends is called treadmilling (Margolis \& Wilson, 1998). Depolymerization which leads to microtubule shrinkage is termed catastrophe whereas rescue describes the process of polymerization resulting in growth of microtubules. The behavior of switching between phases of growth and shrinkage is termed dynamic instability and represent a major characteristic of microtubules (Fig. 1.3) (T. Mitchison \& Kirschner, 1984). The transition between growth and shrinkage can be regulated by microtubuleassociated proteins (MAPs). Among them, MAP1B, which is well known for its microtubule stabilizing function likely by decreasing depolymerization of microtubules (Halpain \& Dehmelt, 2006). Likewise, the proteins of the MAP2/Tau family stabilize microtubules by reducing the occurrence of catastrophes (Dehmelt \& Halpain, 2004). MAPs can be sub divided into plus-tip interacting proteins (+TIPs), polymerases and depolymerases (Howard \& Hyman, 2009). A prominent microtubule polymerase is ch-TOG (colonic and hepatic tumor overexpressed gene; with the corresponding gene name CKAP5), which binds to microtubule plus-ends and catalyzes the addition of tubulin subunits to the growing microtubule (Brouhard et al., 2008). Furthermore, it was revealed that ch-TOG also acts as a microtubule nucleation factor (Thawani et al., 2018). In contrast to ch-TOG, MCAK (mitotic centromere-associated kinesin) leads to microtubule depolymerization by promoting dissociation of tubulin subunits at plus-ends (Hunter et al., 2003). Moreover, stathmin (also called oncoprotein 18/Op18) is known to destabilize microtubules by sequestering tubulin subunits thereby facilitating catastrophe. Additionally, it is hypothesized that stathmin promotes depolymerization by binding directly to microtubules whereby it binds 
preferentially to minus-ends (Gupta et al., 2013). Both, stathmin and ch-TOG were found to be overexpressed in human cancer (Belletti \& Baldassarre, 2011; Charrasse et al., 1995). Especially overexpression of stathmin is associated with invasion, metastasis and poor survival (Belletti \& Baldassarre, 2011).
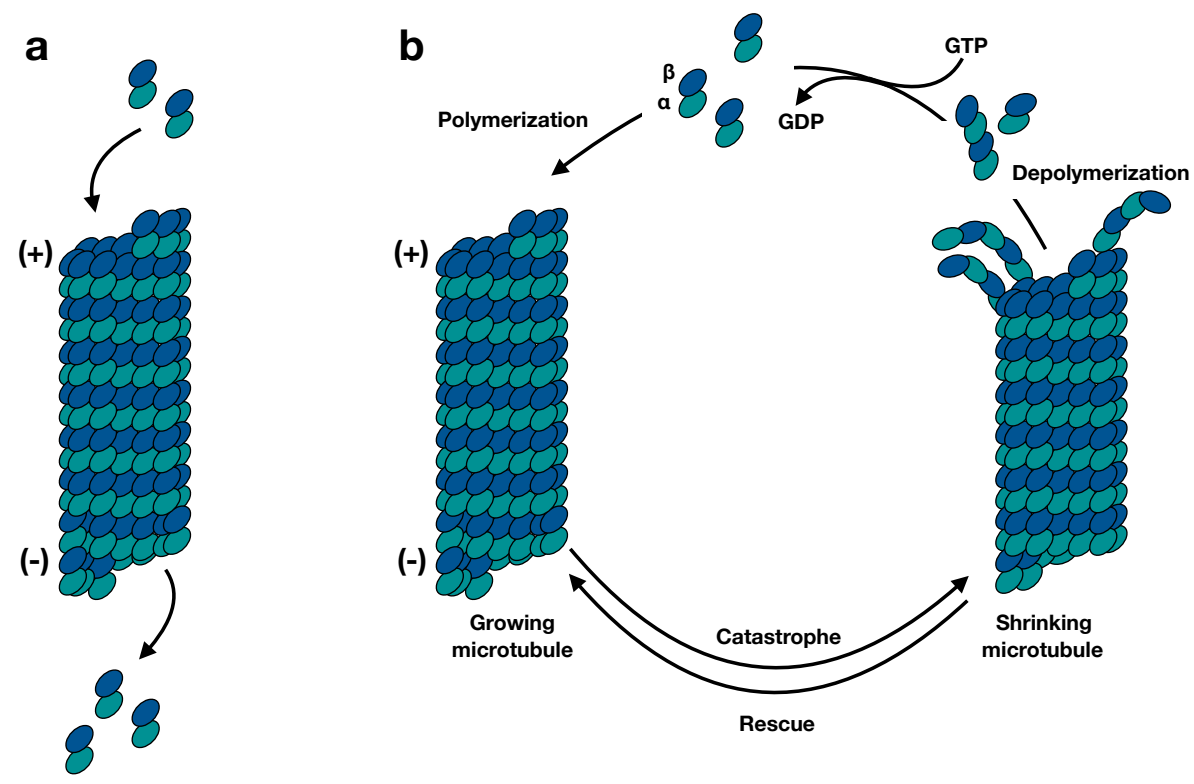

Fig. 1.3 Dynamic instability of microtubules

(a) Microtubule treadmilling. The constant addition of tubulin heterodimers at the (+)-end and loss of tubulin heterodimers at the (-)-end while not affecting microtubule length is termed treadmilling. (b) Microtubule polymerization results from the addition of GTP bound $\alpha$-tubulin and $\beta$-tubulin heterodimers. The addition of heterodimers leads to hydrolysis of GTP- $\beta$-tubulin. Phases between growing and shrinking of microtubules are called rescue in case of polymerization while catastrophe described the process of microtubule depolymerization. Modified from Akhmanova \& Steinmetz, 2015

+TIPs represent another class of MAPs and are characterized by binding and accumulating at plus-ends and involve various proteins. Among them, end-binding proteins (EBs), which are capable of binding directly to growing microtubule plus- and minus-ends. EB1 itself promotes microtubule polymerization and is able to recruit and bind additional +TIPs thereby modulating microtubule dynamics (J. Liu \& Han, 2015). For example, binding of EB1 to CLIP-170 (cytoplasmic linker protein 170)(Goodson et al., 2003) and CLASP (CLIP-associating protein) (Mimori-Kiyosue et al., 2005) leads to stabilization of microtubules, likely by reducing catastrophes and increasing frequencies of rescues (Akhmanova \& Steinmetz, 2015). Moreover, interaction of EB1 with APC (adenomatous polyposis coli) was shown to be important for spindle dynamics and proper alignment of chromosomes thereby influencing chromosomal stability. Intriguingly, APC mutations which lead to impaired EB1 - APC interaction are often observed in colorectal cancer (Green et al., 2005). 


\subsubsection{The actin cytoskeleton}

The actin cytoskeleton plays a crucial role for several processes such as cell shape, providing a contractile cell cortex, cell motility and assembly of a contractile ring for cytokinesis (Schmidt \& Hall, 1998). Actin is present as monomeric G-actin or polymerized F-actin. Spontaneous polymerization occurs very slowly and involves the formation of an actin dimer, which evolves into a trimer. Both intermediates are quite unstable while addition of further actin monomers leads to stabilization of the growing filament (Sept \& McCammon, 2001). During polymerization of F-actin, ATP bound G-actin is hydrolyzed whereby phosphate release occurs slowly, which leads to a long-lasting intermediate of ADP- $P_{i}$ bound actin (Pollard, 2016). The resulting filament reveals a polarity with a barbed (+)-end at which ATP bound actin is present and a disassembling, pointed (-)-end exposing ADP bound actin (Hohmann \& Dehghani, 2019). This process is called treadmilling and is regulated by actin binding proteins (Fig. 1.4) (Bugyi \& Carlier, 2010).
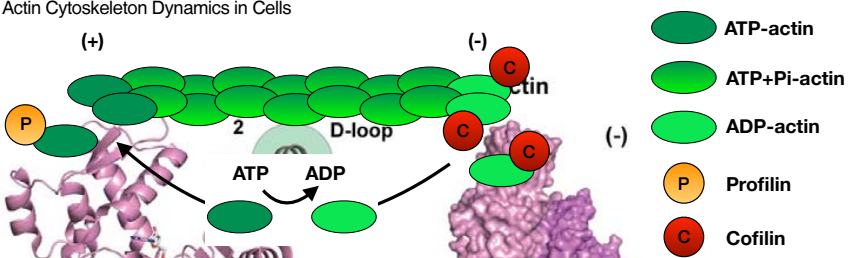

\section{Fig. 1.4 Actin treadmilling}

Polymerization of F-actin from GTP-bound actin monomers, also called G-actin, is accompanied by GTP hydrolysis and primarily occurs at (+)-ends while the (-)-end is characterized by dissociation of ADP-actin. Profilin and cofilin regulate actin treadmilling by binding actin whereby profilin promotes polymerization and cofilin leads to depolymerization at pointed ends. Modified from Lee \& Dominguez, 2010

One of them is profilin, which supports polymerization at barbed ends while actin nucleation and polymerization is suppressed at (-)-ends (Goode \& Eck, 2007; Pollard, 2016). Moreover, profilin catalyzes nucleotide exchange of depolymerized actin bound to ADP thereby increasing the pool of ATP bound actin, which is used by elongation factors to promote actin polymerization (Pollard, 2016). Another actin-binding protein is thymosin- $\beta 4$, which sequesters actin monomers to prevent their incorporation into the growing filament. Moreover, thymosin- $\beta 4$ competes with profilin for binding of actin (Safer \& Nachmias, 1994). The function of the depolymerizing factor ADF/cofilin depends on its concentration. At low concentrations ADF/cofilin binds preferentially to ADP bound F-actin and leads to severing of F-actin and dissociation of actin monomers from pointed ends (Van Troys et al., 2008; Winterhoff \& Faix, 2015). In case of high abundance, ADF/cofilin binds to and stabilizes actin dimers, an intermediate of spontaneously actin polymerization, which promotes actin nucleation (Andrianantoandro \& Pollard, 2006). Additionally, capping protein (CP) 
binds to growing barbed ends and prevents further polymerization but also dissociation of actin monomers (Edwards et al., 2014).

\section{Actin nucleators}

Since it is not energetically favored to polymerize free G-actin, nucleators such as the actin-related protein 2/3 (Arp2/3) complex and formins promote de novo nucleation (Campellone \& Welch, 2010). The latter is able to nucleate and elongate unbranched actin filaments (Goode \& Eck, 2007). In mammals, 15 different formins exist, which all share a FH2-domain (formin homology domain) and can be subdivided into seven classes, namely Dia (diaphanous), DAAM (disheveledassociated activator of morphogenesis), delphilin, FHOD (formin homology domain-containing protein), FMN (formin), FRL (formin-related gene in leukocytes) and INF (inverted formin) (Higgs \& Peterson, 2005). Adjacent to the $\mathrm{FH} 2$ domain, a $\mathrm{FH} 1$ domain is located at the C-terminus. Whereas the $\mathrm{FH} 2$ domain forms a dimer at barbed ends of actin filaments and is required for actin nucleation, FH1 domains bind to profilin bound actin thereby allowing nucleation (Higgs, 2005; Sagot, Rodal, Moseley, Goode, \& Pellman, 2002). Moreover, FH2 domains are capable of moving with the growing actin filament and protecting it from CP thereby promoting polymerization (Pruyne et al., 2016; Zigmond et al., 2003). Some formins appear to have additional functions besides actin nucleation and elongation like bundling or depolymerization of F-actin (Chesarone et al.,2010). In mDia (mammalian dia) proteins, the $\mathrm{FH} 2$ domain enables interaction with microtubules by binding to +TIPs like EB1, APC and CLIP-170, which influences both, microtubule stabilization and actin polymerization (Bartolini et al., 2008; Lewkowicz et al., 2008; Wen et al., 2004). Usually, formins are in an autoinhibited state due to the binding of the dia autoregulatory domain (DAD) at the Cterminus to the dia inhibitory domain (DID) at the N-Terminus. Upon binding of Rho to the GTPase binding domain (GBD) the inhibitory connection is disrupted and results in activation (Li \& Higgs, 2003; Wallar et al., 2006). However, Rho binding is not sufficient to completely resolve autoinhibition (Li \& Higgs, 2005). Thus, additional mechanisms, such as post-translational modifications or additional proteins interacting directly with DID, DAD or with Rho, might be required for activation of formins (Chesarone et al., 2010). Moreover, some formins are not regulated by DAD and DID autoinhibition, but it is likely that proteins such as DIP (dia-interacting protein) bind to $\mathrm{FH} 2$ domains thereby preventing actin polymerization (Chesarone et al., 2010). In contrast to formins, nucleation and elongation by the Arp2/3 complex results in branched actin polymerization emerging from already existing actin filaments. The Arp2/3 complex consists of seven subunits, namely Arp2 and Arp3, which are stabilized by the additional subunits ARPC1-5 (Arp2/3 complex subunit) (Pollard, 2007). It was shown that Arp2 and Arp3 assemble into a dimer, 
which is used as a template for elongation. Additionally, ARPC2-4 are required for initial binding of Arp3 to the mother actin filament whereas ARPC5 promotes branched nucleation by stabilization of Arp2 and by maintaining attachment to the mother filament. ARPC1 is assumed to interact with NPFs (nucleation-promoting factors) (Rouiller et al., 2008). Due to its intrinsically inhibited state, the Arp2/3 complex requires activation by NPF such as the Wiskott-Aldrich Syndrome protein (WASp) or WAVE (WASP family verprolin homologue protein). These proteins harbor VCA domains (verprolin homology, cofilin homology and acidic domain) that enable binding of G-actin on the one hand and Arp2/3 on the other hand, thereby facilitating binding to an existing actin filament and the nucleation of a new, branched actin filament at an angle of approximately $70^{\circ}$ (Mullins et al.,1998; Rotty et al., 2013). Both NPFs appear to be intrinsically inhibited due to masking of the VCA domain, which prevents binding of the Arp2/3 complex. Upon binding of small GTPases, such as Rac1 and Cdc42, the VCA domain is exposed due to conformational changes and able to bind and activate the Arp2/3 complex (Derivery \& Gautreau, 2010).

In contrast to actin nucleators, crosslinker proteins influence actin network architecture without interfering with actin polymerization. Filamin and $\alpha$-actinin are large crosslinkers, which lead to organization of an actin network whereas short crosslinkers like fimbrin or fascin result in compact parallel or antiparallel organized actin structures (Blanchoin et al., 2014). Moreover, myosin itself functions as a crosslinker and leads to contraction of antiparallel organized actin bundled actin filaments, which is required for cytokinesis and stress fibers (Blanchoin et al., 2014; Stam et al., 2015). Additionally, myosin was identified to induce actin depolymerization in a concentration dependent manner (Haviv et al., 2008).

Rearrangement of the actin cytoskeleton plays an important role during cell migration, which basically relies on the repetition of the following steps: formation of membrane protrusions and new adhesions sites at the leading edge and subsequent contraction and retraction of the trailing edge (Etienne-Manneville, 2013). While formation of membrane protrusions, such as lamellipodia and filopodia, are promoted by actin polymerization, the rear is characterized by the contraction of stress fibers (Blanchoin et al., 2014). The formation of lamellipodia and invadopodia requires Arp2/3 mediated actin polymerization while formins are necessary for formation and maintenance of filopodia (Block et al., 2008; Schirenbeck et al., 2005). Thus, the Arp2/3 complex and formins play an important role in cellular migration (Yamaguchi \& Condeelis, 2007). Accordingly, overexpression of the Arp2/3 complex is associated with tumorigenesis of gastric cancer (Zheng et al., 2008). Likewise, it was shown that formins promote invasiveness and metastasis (Lizárraga et al., 2009). Furthermore, analysis of samples from pancreas and brain tumors revealed mutations in formin genes (Jones et al., 2008; Parsons et al., 2008) while additional studies 
showed that enhanced expression of FRL2 promotes metastasis in colorectal cancer (Zhu et al., 2008). At the onset of mitosis, dynamic rearrangements of the actin cytoskeleton occurs due to mitotic rounding, which was shown to be required for spindle assembly, spindle pole integrity and timely mitotic progression (Lancaster et al., 2013). Those changes involve among others, disassembly of actin stress fibers, retraction of the rear and an increase in cortical tension (Maddox \& Burridge, 2003; T. J. Mitchison, 1992). However, actin-rich retraction fibers still ensure attachment of mitotic cells to the substratum (T. J. Mitchison, 1992), which help to orient the mitotic spindle (Théry et al., 2005). Interestingly, Mitsushima and colleagues revealed the formation of subcortical actin clusters, which exhibited a dynamic behavior likely due to ongoing actin polymerization and depolymerization. These so-called actin clouds appeared to be Arp $2 / 3$ dependent and were primarily observed in completely rounded cells throughout mitosis (Mitsushima et al., 2010). Moreover, Fink et al. revealed that the position of retraction fibers leads to polarized polymerization of actin clouds, which were shown to guide spindle orientation (Fink et al., 2011). In line with these findings, it was shown that myosin 10 acts as a linker between actin clouds and astral microtubules to position the mitotic spindle (Kwon, Bagonis, Danuser, \& Pellman, 2015).

\section{Regulation of actin polymerization by the Ras homologous (Rho) family of small GTPases}

Actin nucleators such as mDia1 and the Arp2/3 complex are regulated by the Ras homologous family of small GTPases (Rho GTPases), which play a major role in actin regulation (Hall, 1998; Lee \& Dominguez, 2010). Rho GTPases are also known as molecular switches since they alternate between an active state and an inactive state to regulate the activity of downstream effectors (Mackay \& Hall, 1998). When bound to GTP, Rho GTPases are in an active state whereas hydrolysis to GDP leads to inactivation (Fig. 1.5). Three types of proteins are known to control cycling between an active and inactive state. So-called guanine nucleotide exchange factors (GEF) enable the dissociation of GDP from Rho GTPases and thereby facilitating the binding of GTP (Y. Zheng, 2001) whereas GTPase activating proteins (GAP) lead to an increase of GTPase activity thus promoting GTP hydrolysis (Bos, Rehmann, \& Wittinghofer, 2007). Guanine nucleotide dissociation inhibitors (GDI) bind to GDP-Rho, prevent nucleotide exchange and are considered to regulate localization of Rho proteins (Dransart et al., 2005). 


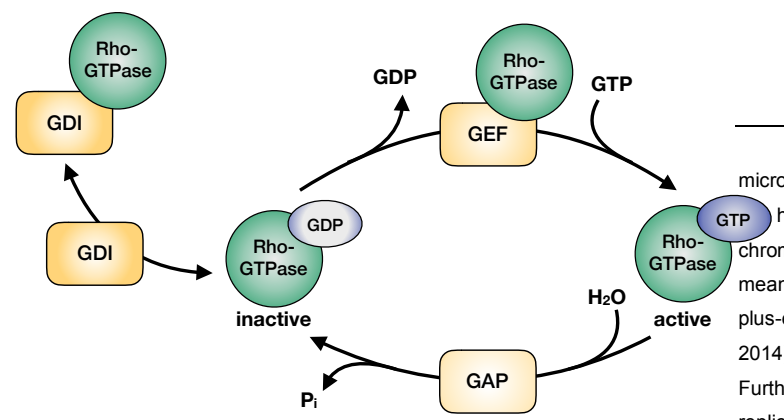

Fig. 1.5 Regulation of Rho GTPases by GEF, GAP and GDI

Rho GTPases are activated via GEFs, which promote the dissociation of GDP thereby leading to activation. Inactivation is promoted by GAPs due to their ability to promote GTP hydrolysis. Moreover, GDI prevent nucleotide exchange upon binding and regulate localization of Rho GTPases. Modified from Rossman et al., 2005.

The most prominent members of Rho GTPases are RhoA, Rac1 and Cdc42. Activation of the Arp2/3 complex is mediated by Rac1 and Cdc42 whereby Rac1 leads to WAVE activation while Cdc42 results in WASP mediated activation of the Arp2/3 complex. Furthermore, Rac1 and Ccd42 activate the p21-activated kinase (PAK), IQGAP1 (IQ motif containing GTPase activating protein) and IQGAP2 (Kuroda et al., 1999). RhoA promotes actin rearrangements via activation of mDia while ROCK (Rho-associated kinase) activates myosin II (Burridge \& Wennerberg, 2004; Narumiya et al., 2009) and LIM-kinase (LIMK), which inhibits ADF/cofilin. Thus, RhoA mediated ROCK activation results in actomyosin based contraction and stabilization of actin filaments (Maekawa et al., 1999). Additionally, PAK is also able to phosphorylate LIMK thereby promoting actin stabilization (Fig. 1.6) (Hanna \& El-Sibai, 2013).

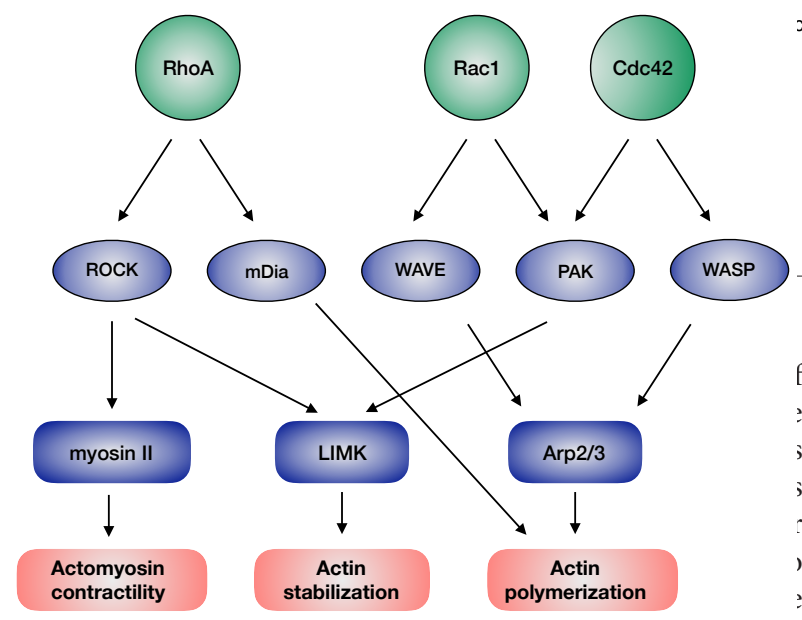

Fig. 1.6 Regulation of the actin cytoskeleton by Rho GTPases

RhoA activates ROCK and mDia. While ROCK promotes actomyosin based contraction via activation of myosin II, phosphorylation of LIMK results in actin stabilization. Additionally, mDia leads to actin polymerization. Both, Rac1 and Cdc42 activate PAK, which promotes actin stabilization via LIMK. Arp2/3 
mediated actin polymerization is achieved by Rac1 and Cdc42, whereby the latter activates WASP while Rac1 leads to WAVE activation. Modified from Hanna \& El-Sibai, 2013.

Rho GTPases are well-known for their implication in cell migration (Nobes \& Hall, 1999) during which Rac1 and Cdc42 promote Arp2/3 mediated branched actin polymerization via activation of WAVE and WASP at the leading edge to form membrane protrusions (Blanchoin et al., 2014). These are stabilized by newly formed adhesions mediated by Rac1 during early and RhoA during late adhesion (Lawson \& Burridge, 2014). At the rear of the cell, RhoA activates ROCK which results in actomyosin based contraction thereby facilitating movement towards the leading edge (Narumiya et al., 2009; Worthylake et al.,2001). Especially during migration, Rac1 and RhoA influence each other's activity by several GEFs and GAPs (Fig. 1.7).

For example, Rac1 mediated PAK activation inhibits several Rho GEFs, such as GEF-H1 and p115-RhoGEF, thereby preventing RhoA activation. Likewise, phosphorylation of p190RhoGAP by Rac1 suppresses RhoA activity. In contrast, activation of the Rho GEF Dbs by Rac1 promotes RhoA activation. Vice versa, dependent on its downstream effector, ROCK is able to prevent or promote Rac1 activation (Guilluy, Garcia-Mata, \& Burridge, 2011). Additionally, mDia promotes Rac1 activation whereby high activity of ROCK prevents mDia mediated Rac1 activation (Arakawa et al., 2003).

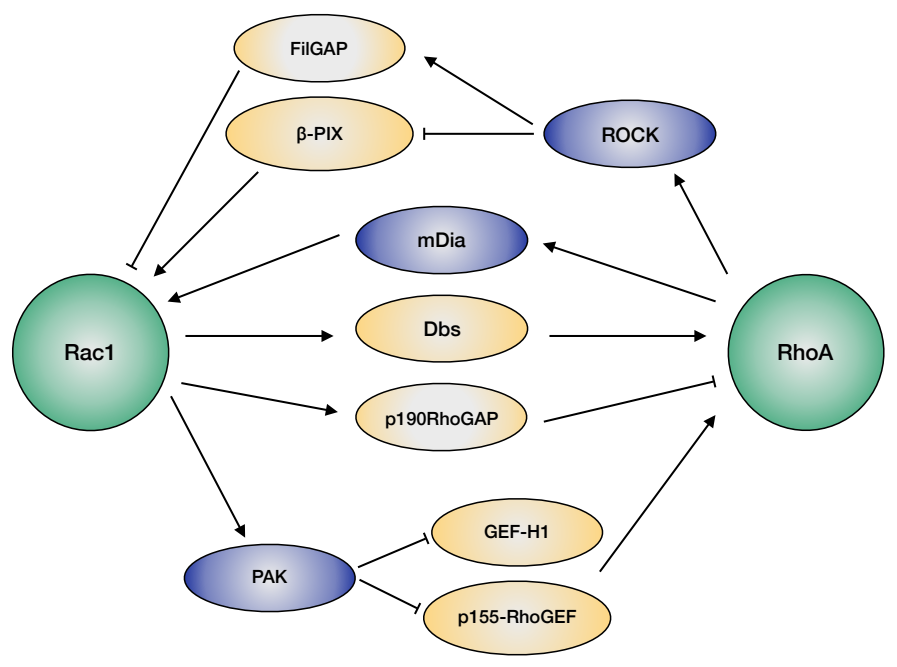

Fig. 1.7 Crosstalk between Rac1 and RhoA

Rac1 is able to prevent RhoA activation via PAK or directly by activating p190RhoGAP whereas Dbs leads to Rac1 induced RhoA activation. Vice versa, downstream of RhoA, mDia and ROCK are able to promote Rac1 activation while FilGAP prevents Rac1 activation. Modified from Guilluy et al., 2011.

Besides a role in cell migration, Rac1 and Cdc42 appear to promote cell cycle progression by enhancing cyclin D expression and altering p21 levels (Bao, et al., 2002; Joyce et al., 1999). The latter is known as Cdk inhibitor, which binds to and inactivates cdks thereby preventing transition 
from $\mathrm{G}_{1}$ to S-phase (Gartel, Serfas, \& Tyner, 1996). Moreover, RhoA was shown to suppress p21 to overcome Ras induced cell cycle arrest (Olson, Paterson, \& Marshall, 1998). Interestingly, van Haren et al. identified the Rac1 GEF TRIO as a +TIP since it was shown that it binds to microtubule plus-ends in an EB1-dependent manner and increases Rac1 activity to remodel the actin cytoskeleton in neuros (Van Haren et al., 2014). Moreover, Rac1 was found to be activated by TIAM1 (T-lymphoma invasion and metastasis- inducing protein-1) at centrosomes during mitosis (Woodcock et al., 2010), which resulted in PAK activation (Whalley et al., 2015a). Inhibition of this signaling pathway was identified to influence centrosome separation, which finally resulted in chromosome congression defects (Whalley et al., 2015a).

So far, no mutations or constitutive active Rho GTPases have been found in human tumors. However, overexpression of Rac1, Cdc42 and Rho proteins is associated with tumor progression in various types of cancer (Sahai \& Marshall, 2002). Moreover, overexpression of TRIO and Tiam1 correlates with poor patients outcome (Lane et al., 2008). Likewise, downstream effectors of Rho GTPases, like PAK or IQGAP1 were also reported to be overexpressed in colorectal cancer leading to migration, invasion and metastasis (Leve \& Morgado-Díaz, 2012).

\subsection{Cortical tension}

Downstream of RhoA signaling, nonmuscle myosin II (NM II or myosin II) along with actin filaments is crucial for the generation and regulation of cortical tension and the formation of the contractile ring during cytokinesis. Myosin II is able to bind to actin, which activates ATP hydrolysis by NM II. Class II myosins consist of two heavy chains that form a homodimer, which binds to two essential light chains and a pair of regulatory light chains. (Conti \& Adelstein, 2008). Motor activity and assembly of NM II filaments is regulated via phosphorylation of the regulatory light chain (RLC) and heavy chain (Bresnick, 1999). More precisely, dephosphorylation of RLC results in a conformation of myosin II, which leads to inhibition of its ATPase activity and prevents actin binding (Wendt et al., 2001). Thus, phosphorylation of RLC facilitates actin binding and promotes ATPase activity of myosin thereby leading to actomyosin contraction. Besides ROCK (Rho associated kinase), the myosin light chain kinase (MLCK), citron kinase and MRCK (myotonic dystrophy kinase-related Cdc42-binding kinase) are responsible for phosphorylation of RLC whereas dephosphorylation of RLC by MLCP (myosin light chain phosphatase) leads to inactivation of NM II (Levayer \& Lecuit, 2012; Matsumura \& Hartshorne, 2008). Phosphorylation of MLCP by ROCK leads to its inactivation and further increases phosphorylation of RLC (Fig. 1.8) (Maekawa et al., 1999). 


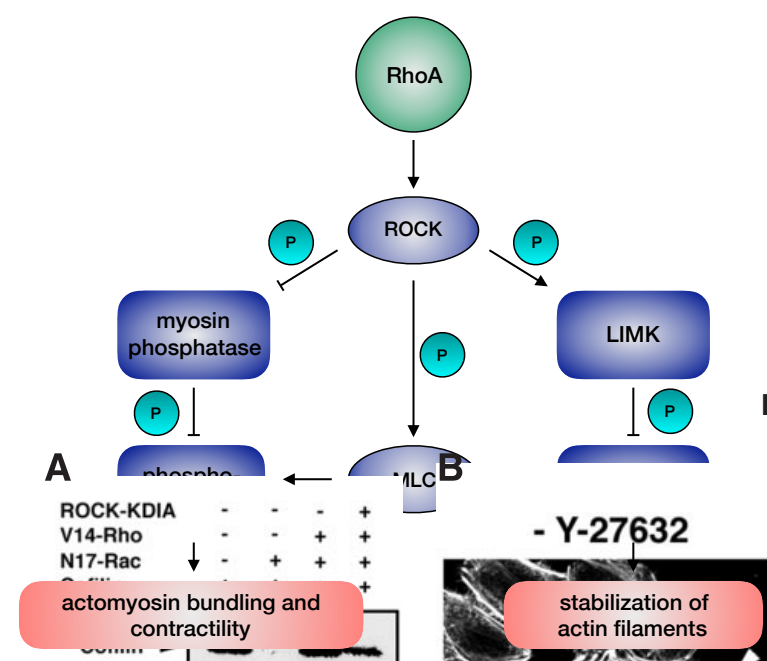

Fig. 1.8 Regulation of actin filament stabilization and actomyosin contractility by RhoA and ROCK ROCK activation by RhoA results in phosphorylation of LIMK on the hand, which inhibits the actin severing function of cofilin, which leads stabilization of actin filaments. On the other hand, ROCK phosphorylates, and thus activates myosin light chain kinase (MLC). Additionally, ROCK prevents dephosphorylation of MLC by phosphorylating myosin phosphatase. Thus, ROCK mediates actin filament stabilization and actomyosin contractility. Modified from Rath \& Olson, 2012.

Contraction of actomyosin is achieved by ATPase activity of NM II. ATP bound myosin II binds to actin after hydrolysis of ATP to ADP and phosphate. Subsequent phosphate release results in a pulling force on actin induced by conformational changes of the myosin head. Subsequently, ADP release allows binding of a new ATP molecule, which results in dissociation of myosin from the actin filament (Sivaramakrishnan et al., 2009).

During cytokinesis, RhoA is activated in a cell cycle dependent manner by several GEFs, such as Ect2 (epithelial cell transforming 2) and GEF-H1, which leads to unbranched actin polymerization via formins and to ROCK mediated myosin activation thereby ensuring assembly and contraction of the contractile ring, which finally results in membrane ingression (Fededa \& Gerlich, 2012). Another function of myosin II during mitosis involves the so-called cortical flow within the cell cortex, which is based on actomyosin contraction and leads to movement of F-actin, myosin II and cortex associated proteins (Benink, Mandato, \& Bement, 2000; Bray \& White, 1988). Rosenblatt and colleagues revealed that cortical flow is responsible for centrosome separation after breakdown of the nuclear envelope, which indicates a role for myosin II in mitotic spindle assembly (Rosenblatt et al., 2004). In mouse oocytes, myosin II was identified to localize at meiotic spindle poles, which was crucial for spindle relocation at the cell cortex to ensure asymmetric cell division (Schuh \& Ellenberg, 2008). Moreover, myosin 10 is able to bind directly to microtubules and is necessary for spindle assembly in X. laevis (Weber et al., 2004). Supporting this, Kwon et al. 
demonstrated that myosin 10 facilitates centrosome separation by coupling astral microtubules to subcortical actin clouds thereby guiding spindle positioning (Kwon et al., 2015).

Mitotic rounding was shown to be driven by hydrostatic pressure and cortical tension, which is mainly based on actomyosin contraction (Salbreux, Charras, \& Paluch, 2012; Stewart et al., 2011). Interestingly, Chugh and colleagues demonstrated that mitotic cells exhibit a thinner cortex but higher tension than cells in interphase (Chugh et al., 2017). Furthermore, unbranched actin structures mediated by formins result in higher tension whereas branched actin filaments by Arp2/3 are more rigid but less contractile (Levayer \& Lecuit, 2012). Supporting this, RhoA mediated actin nucleation by mDia1 rather than Arp2/3 induced actin polymerization is responsible for generation of cortical tension (Ramanathan et al., 2015). Additionally, Chugh et al. revealed that the actin filament length regulators $\mathrm{mDia}$ in concert with ADF/cofilin and barbed end-capping protein regulate surface tension by modulating cortex thickness without affecting myosin II localization. Thus, an increase or decrease of cortex thickness resulted in lower tension of mitotic cells whereby actin filament length rather than cortex thickness per se might be the critical factor (Chugh et al., 2017). These results suggest, that besides myosin II activity, actin cortex structure has to be finely regulated in order to maintain proper cortex tension.

Intriguingly, it was found that cancer cells derived from patients behave less rigid in comparison to cells from healthy donors (Cross et al., 2007; Remmerbach et al., 2009). These findings indicate that cortical tension might play a role for disease development and progression.

\subsection{Crosstalk between microtubule and actin cytoskeleton}

Especially in terms of migration, Rho GTPases represent an important link between the actin and microtubule cytoskeleton (Wojnacki et al., 2014). Migrating cells reveal a polarization due to growing microtubules in the direction of the leading edge whereas depolymerizing microtubules are present at the rear of the cell (Kaverina \& Straube, 2011). The former induces Rac1 activation, which promotes actin polymerization thereby facilitating formation of membrane protrusions like lamellipodia (Waterman-Storer et al., 1999). Moreover, +TIPs such as CLIP-170 (Fukata et al., 2002) and APC (T. Watanabe et al., 2004) were shown to bind the Rac1 and Cdc42 effector IQGAP1, which promotes Arp2/3 and formin mediated actin polymerization at the leading edge thereby promoting formation of membrane protrusions (Brandt \& Grosse, 2007). Conversely, microtubule depolymerization mediates RhoA activation, likely by GEF-H1, which is released upon microtubule depolymerization and thus able to activate RhoA thereby leading to the formation of stress fibers via formins. Additionally, ROCK activation results in contraction of stress fibers to promote forward movement (Watanabe et al., 1999; Watanabe et al., 1997). Vice versa, Rac1 and 
Cdc42 appear to influence microtubule dynamics by phosphorylation of stathmin via their downstream effector PAK, which led to inactivation of stathmin thereby preventing microtubule depolymerization (Daub et al.,2001). Additionally, mDia1 was shown to stabilize microtubules by binding EB1 and APC to promote migration (Wen et al., 2004) while growing microtubules were shown to induce actin nucleation via binding of mDia1 by CLIP-170 (Henty-Ridilla et al., 2016).

Cytokinesis represent another process in which crosstalk between the actin and microtubule cytoskeleton is important. Here, one can distinguish between three types of microtubules, namely, astral and polar microtubules and bundled, antiparallel organized microtubules between spindle poles, which form the central spindle (Chircop, 2014). While cortical localized stable microtubules near the cell equator are considered to promote contractility, more dynamic astral microtubules suppress contractility at the spindle poles, which leads to localization of active RhoA and myosin II at the cleavage furrow (Bement, Benink, \& Von Dassow, 2005; Foe \& Von Dassow, 2008; Werner, Munro, \& Glotzer, 2007).

Another link between the actin and the microtubule cytoskeleton was identified by van Haren et al. who demonstrated that TRIO in a complex with Nav1 (Neuron Navigator 1) binds to EB1 at microtubule plus-ends which induced actin rearrangement via activation of Rac1 in neurons. Moreover, this process was shown to be dependent on dynamic microtubules (Van Haren et al., 2014).

However, those interactions play a role in interphase cells. In mitosis, so far, myosin 10, MISP (mitotic interactor and substrate of Plk1) and ERM (ezrin, radixin, moesin) proteins link microtubules to the actin cytoskeleton. Since all of them play a role in spindle orientation, they will be described in more detail in the following chapter (Dogterom \& Koenderink, 2019).

\subsection{The mitotic spindle}

\subsubsection{Spindle assembly}

The structure, which ensures faithful chromosomes segregation is called the mitotic spindle. It mainly consists of microtubules, MAPs and motor proteins. One can distinguish between three different classes of microtubules. Kinetochore microtubules that attach to chromosomes by binding to kinetochores are called k-fibers when bundled. Astral microtubules extend towards the cell periphery and contribute to anchor and position the spindle at the cell cortex. Microtubules emanating from the centrosomes towards the center of the cell are called interpolar (Forth \& Kapoor, 2017). The term microtubule organization center (MTOC) defines a structure, which is responsible for catalyzing the $y$-tubulin dependent nucleation, anchorage and organization of microtubules (Lüders \& Stearns, 2007). During mitosis, centrosomes represent the main MTOC 
responsible for spindle assembly whereby chromosome-derived signals and augmin represent centrosome independent options for spindle assembly (Forbes et al., 2015; Goshima et al., 2008; Meraldi, 2016).

\section{Centrosome dependent spindle assembly}

The centrosome consists of two centrioles, which are linked by fibers, orthogonal oriented towards each other and surrounded by PCM (pericentriolar material) (Urbani \& Stearns, 1999). The latter is composed of several proteins whereby $y$-tubulin is essential for nucleation activity and forms with members of the gamma complex protein family the $\mathrm{y}$-tubulin ring complex ( $\mathrm{y}$-TuRC), which serves as a template for microtubule nucleation (Mourey, Agard, Kollman, \& Merdes, 2011). In order to establish a bipolar spindle, the centrosome has to be duplicated and sister centrosomes have to be separated (Fig. 1.9). During $G_{1}$, disjunction of the two centrioles starts to allow centrosome duplication in S-phase, which involves formation of new daughter centrioles and their elongation (Urbani \& Stearns, 1999). Centrosome maturation describes the expanding PCM, which is necessary for proper organization of microtubules and takes place in late $\mathrm{G}_{2}$. This process requires AuroraA kinase and Plk1 at the centrosome (Hannak et al., 2001; Lane \& Nigg, 1996; Lee \& Rhee, 2011). Subsequent centrosome separation is divided into two phases, namely disjunction and movement. These processes, disjunction and movement of centrosomes towards spindle poles, are also termed centrosome dynamics (Nam, Naylor, \& van Deursen, 2015).

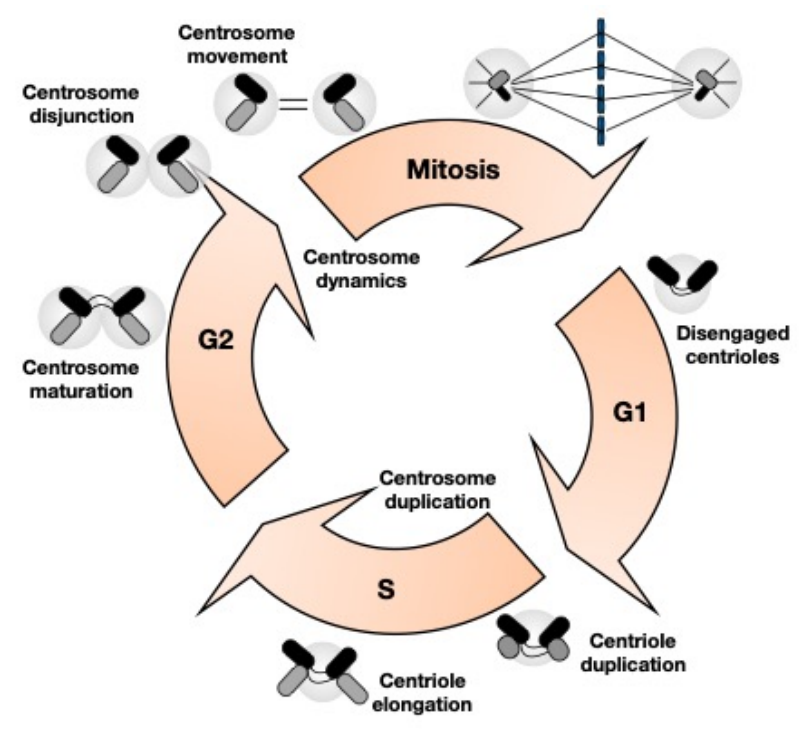

Fig. 1.9 The centrosome cycle

Centriole disjunction is required in $\mathrm{G}_{1}$ to allow duplication and elongation of centrioles in S-phase. During $\mathrm{G}_{2}$, centrosome maturation takes place, which involves PCM expansion. Afterwards, the cohesion linker is dissolved, and thus the centrosomes start to separate. Centrosome disjunction and movement are summarized under the term centrosome dynamics. Modified from Nam et al., 2015. 
Centrosome disjunction is initiated at $\mathrm{G}_{2}-\mathrm{M}$ transition by $\mathrm{Cdk} 1$ in complex with cyclin B2, which phosphorylates AuroraA, which in turn phosphorylates and activates Plk1. Finally, Plk1 mediates phosphorylation of a cohesion complex, which connects sister centrosomes, thereby leading to centrosome disjunction. Kinesins are essential for the process of centrosome movement. These are motor proteins, which utilize ATP hydrolysis to move along microtubules (Vicente \& Wordeman, 2015). Together with Kif2A (kinesin family) and KifC1, Eg5 (also called Kif11) is the driving force for centrosome movement and bipolar spindle assembly (Zhu et al., 2005). Eg5 is a plus-end directed kinesin and binds as a tetramer to microtubules organized in an antiparallel manner and moves along microtubules thereby pushing them apart (Mann \& Wadsworth, 2019). This process is also initiated by Plk1, which triggers a signaling cascade resulting in Eg5 phosphorylation and activation at the centrosome. Additionally, Cdk1 might also directly activate Eg5 to promote centrosome separation (Nam et al., 2015; Smith et al., 2011). It was reported that centrosome separation occurs during prophase along the nuclear envelope or after nuclear envelope breakdown (NEBD) in prometaphase (Tanenbaum \& Medema, 2010). In prophase, Eg5 was shown to separate centrosomes (Tanenbaum \& Medema, 2010). Another class of motor proteins, namely dyneins exhibit minus-end directed movement and can be categorized into a cortical, astral microtubule and nuclear envelope localized pool (Kardon \& Vale, 2009; Raaijmakers et al., 2012). The latter was shown to promote centrosome separation by pulling them along the nucleus (Raaijmakers et al., 2012). Thus, in prophase Eg5 and dynein act in concert to separate centrosomes (Van Heesbeen et al., 2013). Another factor that plays a role during prophase is the force generated by microtubules. Based on polymerization of microtubules in close proximity, encountering pushes microtubules away from each other thereby promoting centrosome separation (Cytrynbaum et al., 2003; Dogterom et al., 2005).

After NEBD, it was shown that the connection of astral microtubules to the cell cortex and a cortical flow is gaining in importance. Rosenblatt et al. revealed that myosin II and cortical actin are required for centrosome separation and complete spindle assembly after NEBD (Rosenblatt et al., 2004). Moreover, results from De Simone et al. indicate that in C. elegans cortical dynein acts as a coupling device between astral microtubules and cortical flow to separate centrosomes and promote bi-polar spindle assembly (De Simone, Nédélec, \& Gönczy, 2016).

\section{Acentrosomal spindle assembly}

One can distinguish between two pathways leading to centrosome independent spindle assembly. One mechanisms is dependent on chromosomes, which involves RanGTP (Ras-related nuclear 
protein) and the CPC (chromosomal passenger complex), while the other mechanism relies on microtubules and the augmin complex (Meunier \& Vernos, 2016).

During interphase, Ran is localized in the nucleus along with its GEF RCC1 (regulator of chromosome condensation 1), which is only active when bound to chromatin whereas the Ran GAP1 is localized in the cytoplasm (Prosser \& Pelletier, 2017). In mitosis, the different localization of RCC1 and RanGAP1 leads to a RanGTP gradient with a high concentration at chromosomes decreasing towards the cytoplasm. Active RanGTP results in activation of spindle assembly factors, which finally recruit $\mathrm{y}$-TurC thereby promoting microtubule nucleation at kinetochores (Forbes et al., 2015). Additionally, microtubules generated by RanGTP are stabilized by the CRC (Meunier \& Vernos, 2016). AuroraB kinase, as a part of the CRC (Carmena et al., 2012) phosphorylates the microtubule depolymerizing factors stathmin and MCAK, thereby leading to microtubule stabilization at kinetochores (Kelly et al., 2007; Sampath et al., 2004).

Acentrosomal, microtubule dependent nucleation relies on the augmin complex, which comprises eight subunits and binds directly to microtubules (Sánchez-Huertas \& Lüders, 2015). By recruiting $\mathrm{Y}$-TurCs to pre-exisiting microtubules, augmin is able to amplify microtubule nucleation within the mitotic spindle (Kamasaki et al., 2013). Even though augmin and RanGTP are two independent mechanisms, augmin mediated microtubule nucleation is promoted by RanGTP (Meunier \& Vernos, 2016). Microtubules polymerized within the mitotic spindle are organized into a bipolar spindle by Eg5 and dynein. While Eg5 pushes microtubules at the cell equator towards spindle poles, dynein leads to localization of microtubules minus-ends to spindle poles (Lecland \& Lüders, 2014). Finally, it was reported that acentrosomal and centrosome dependent pathways for spindle assembly act in parallel (Hayward et al., 2014).

\subsubsection{Chromosome alignment}

Already in 1986 Kirschner and Mitchison came up with the hypothesis of search and capture to explain chromosome alignment (Kirschner \& Mitchison, 1986). It describes the fact by growing randomly due to dynamic instability, microtubules search for kinetochores, which will be captured and stabilized upon contact thereby facilitating chromosome congression. Several factors influence chromosome alignment at the metaphase plate. Among them, CENP-E and dynein mediated movement of chromosomes and polar ejection forces (PEF) (Heald \& Khodjakov, 2015). Dynein localized at the kinetochore, captures astral microtubules and thus, transports chromosomes towards the spindle poles (Yang et al., 2007). Moreover, PEFs push chromosomes towards the cell equator (Rieder \& Salmon, 1994). These movements rely on the activity of socalled chromokinesins, motor proteins, which are able to bind to microtubules and to chromatin 
(Mazumdar \& Misteli, 2005). By doing so, chromosomes are pushed towards the metaphase plate due to microtubule plus-end directed movement (Wandke et al., 2012; Yajima et al., 2003). Kif4A and Kif18A represent counteracting chromokinesins, which suppress microtubule dynamics to facilitate chromosome congression (Stumpff et al., 2008; Stumpff, et al., 2012). Dynein was shown to inhibit random PEFs and stabilization of incorrect kinetochore-microtubule attachments (Barisic et al., 2014). Finally, CENP-E dominates chromokinesins and dynein to promote transport of chromosomes along microtubules towards the cell equator (Barisic et al., 2014; Yardimci et al., 2008).

\subsubsection{Spindle Orientation}

Following spindle assembly, the mitotic spindle has to be positioned within the cell. Since the spindle axis defines the fate of cell division, spindle orientation is important for asymmetric and symmetric cell division as well as maintenance of tissue architecture in the epithelium. Thus, improper spindle orientation is associated with the development of several diseases (di Pietro, Echard \& Morin, 2016).

The classical pathway for spindle orientation involves a complex consisting of LGN (also called GPSM2; G-protein signaling modulator protein 2), Gai, NuMA1 (nuclear mitotic apparatus protein) and dynein (Fig. 1.10) (di Pietro et al., 2016). LGN was shown to bind the cortical localized heterotrimeric protein $\mathrm{Ga}_{\mathrm{i}}$ via its c-terminal GPR domain ( $\mathrm{G}$ protein regulator) and to NuMA via the n-terminal TPR (tetratricopeptide repeats) domain (Peyre et al., 2011). NuMA in turn is able to bind to microtubules and to dynein (Merdes et al., 1996), which is considered to pull on astral microtubules due to its minus-end directed movement thereby positioning the spindle (Okumura et al., 2018). In interphase and mitosis $\mathrm{Ga}_{i}$ is localized along the cortex, and thus not responsible for specific localization of LGN (di Pietro et al., 2016). While LGN is localized in an inactive state in the cytoplasm, NuMA is restricted to the nucleus during interphase. Upon NEBD, NuMA is released into the cytoplasm and might be able to activate LGN by binding via the N-terminal TPR (tetratricopeptide repeats) domain (Du \& Macara, 2004; Peyre et al., 2011). Cortical localization of LGN and NuMA is regulated by several factors. It was shown that Afadin, which is localized at the cortex, might be responsible for the initial recruitment of LGN by linking cortical F-actin and LGN (Carminati et al., 2016). The authors proposed that upon Afadin mediated recruitment to the cortex, LGN binds to $\mathrm{Ga}_{\mathrm{i}}$ and the following Afadin releas allows NuMA to bind (Carminati et al., 2016). Binding between $L G N$ and $G \alpha_{i}$ was shown to be regulated by the $G \alpha_{i} G E F$ Ric8A and GAP RGS14 since LGN is only able to interact with GDP bound $\mathrm{Ga}_{i}$ (Couwenbergs, Spilker, \& Gotta, 2004; Hess et al.,2004; Willard, Kimple, \& Siderovski, 2004). Another mechanism for polarized 
localization of LGN, NuMA and dynein includes spindle pole and chromosome derived signals. Experiments from Kiyomitsu et al. showed that LGN and NuMA are restricted to areas afar from RanGTP, which leads to spindle pole localization at metaphase. This might be due to the impaired ability of the LGN-NuMA complex to localize at the cell cortex upon exposure to RanGTP. Moreover, Plk1 at spindle poles seems to induce dissociation of dynein from the LGN-NuMA complex given the circumstance spindle poles associated with active Plk1 are in close proximity to the cortex (Kiyomitsu \& Cheeseman, 2012).

\section{Crosstalk between the actin and microtubule cytoskeleton during spindle orientation}

Proteins, which function as a linker between the actin and microtubule cytoskeleton are also essential for proper spindle orientation. As such, MISP was identified, which is an actin-binding protein, regulated in a cell cycle dependent manner by Plk1 (polo-like kinase 1) and binds to EB1 and $\mathrm{p} 150^{\text {glued }}$, a subunit of the dynein-dynactin complex (Akhmanova \& Steinmetz, 2008; Maier et al., 2013; M. Zhu et al., 2013). Moreover, it was revealed that MISP promotes proper spindle orientation by regulating the localization at the cortex of $\mathrm{p} 150^{\text {glued }}$ likely via stabilization of astral microtubules (M. Zhu et al., 2013). Interestingly, Vodicska and colleagues demonstrated that IQGAP1 is a downstream effector of MISP and leads to Cdc42 activation, which was also shown to be required for proper spindle orientation (Hao et al., 2010; Vodicska et al., 2018). Furthermore, IQGAP was responsible for MIPS dependent localization of $\mathrm{p} 150^{\text {glued }}$ and microtubule stabilization (Vodicska et al., 2018).

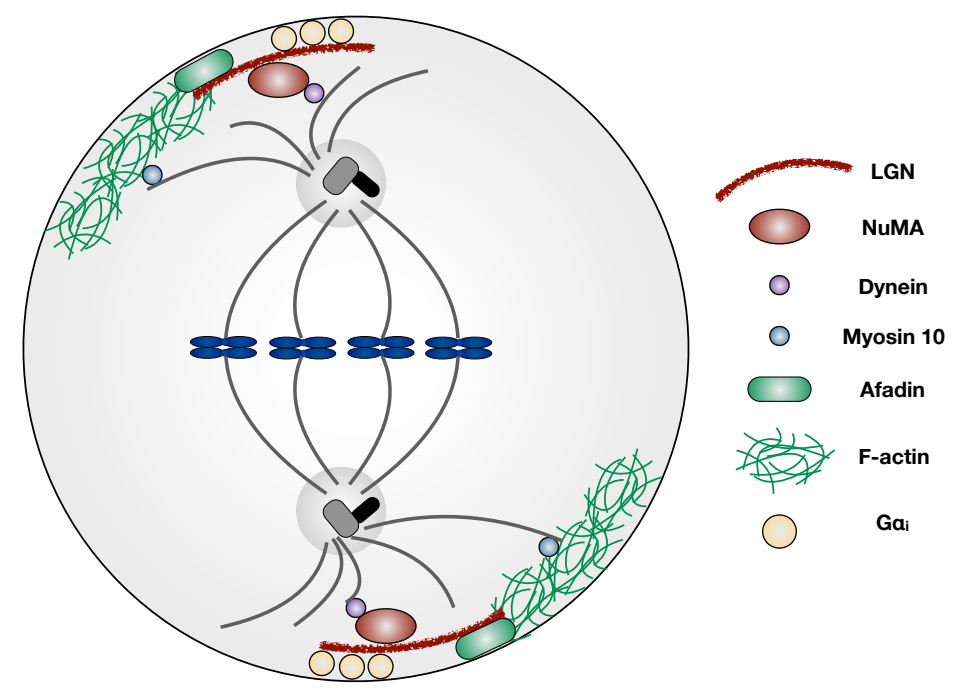

Fig. 1.10 Model for the regulation of mitotic spindle orientation

The NuMA-LGN complex is anchored at the cell cortex via $\mathrm{Ga}_{\mathrm{i}}$. Dynein, which binds to NuMA and astral microtubules moves towards spindle poles, and thus pulls on astral microtubules to position the spindle. Results suggest that Afadin is responsible for initial recruitment of LGN to the cell cortex. Moreover, myosin 
10 was reported to couple astral microtubules to actin clouds thereby promoting spindle orientation. Modified from de Pietro et al., 2016.

Another class of proteins involved in spindle orientation are ERM proteins, which function as crosslinkers between F-actin and the plasma membrane (Bretscher, Edwards, \& Fehon, 2002). Work form Machicoane and colleagues indicates that activated ERM proteins at the cortex regulate localization of LGN and NuMA thereby promoting proper spindle orientation (Machicoane et al., 2014). Moreover, Kunda et al. demonstrated that cortical localized moesin promotes mitotic rounding and cortical rigidity independent of myosin II thereby facilitating proper spindle orientation (Kunda, et al., 2008). The involvement of the actin cortex for spindle orientation was also demonstrated by several groups. Results from Luxenburg et al. indicate that a proper actin cortex and cortical localization of myosin II are required for correct localization of the LGN, Ga $a_{i}$, NuMA and dynein complex (Luxenburg et al., 2011). In line with this, an actomyosin based cortical flow was also shown to be required for proper spindle orientation (Rosenblatt et al., 2004). Additionally, as already mentioned, Arp2/3 dependent actin cloud based on the position of retraction fibers promote spindle positioning (Fink et al., 2011; Mitsushima et al., 2010). Here, myosin 10 was shown to function as a coupling device between astral microtubules and actin clouds thereby facilitating proper spindle orientation (Kwon et al., 2015).

In the context of an epithelium, spindle orientation is crucial for regulating tissue growth and differentiation. While planar spindle orientation leads to symmetric and proliferative cell division, perpendicular alignment results in asymmetric and differentiative cell division (Morin \& Bellaïche, 2011). Accordingly, Spindle misorientation due to loss of tumor suppressor promotes tumorigenesis (Martin-Belmonte \& Perez-Moreno, 2012). Especially precancerous tissue is characterized by loss of asymmetric cell division (Quyn et al., 2010). Moreover, mutated APC, which is described as a main driver for development of colorectal cancer (Powell et al., 1992), was shown to result in defective spindles and chromosome alignment and finally cause chromosomal instability (Fodde, Smits, \& Clevers, 2001; Green et al., 2005).

\subsection{Chromosomal Instability}

Proper mitosis and faithful chromosome segregation represent a prerequisite to ensure an euploid and stable karyotype. However, about $90 \%$ of solid tumors exhibit aneuploidy, a state which describes an aberrant number of chromosomes (Orr, Godek, \& Compton, 2015). One of the mechanisms leading to aneuploidy is chromosomal instability (CIN) (Lengauer, Kinzler, \& Vogelstein, 1997). There are two types of CIN, namely segmental chromosomal instability (S-CIN)

which includes structural rearrangements of chromosomes like deletions, amplifications or translocations whereas the perpetual loss or gain of whole chromosomes is defined as whole 
chromosomal instability (W-CIN) (Ricke, van Ree, \& van Deursen, 2008). It was shown that only $1 \%$ of chromosomally stable cells mis-segregate chromosomes whereas CIN cells reveal a higher rate of chromosome mis-segregation (Lengauer et al., 1997; Thompson \& Compton, 2008). Due to constant chromosome mis-segregation, CIN cells acquire genetic heterogeneity (Heng et al., 2013), which contributes to the adaption of the tumor to its environment thereby facilitating the development of therapy resistance and tumor progression (Gerlinger \& Swanton, 2010; Lee et al., 2011). These aspects of CIN cells are associated with poor patient's prognosis and outcome (Bakhoum et al.,2011; McGranahan et al., 2012). Especially in colorectal cancer cells (CRC), CIN is a major phenotype since approximately $80-85 \%$ of CRC exhibit CIN while only $15 \%$ show microsatellite instability (MIN/MSI). The latter phenotype is characterized by mutations of genes involved in DNA mismatch repair, which give rise to microsatellite instability. In contrast to tumors exhibiting CIN, MSI tumors reveal a good prognosis (Vilar \& Gruber, 2010).

Interestingly, it was shown that high levels of CIN lead to cell death instead of promoting tumor growth whereas a moderate CIN phenotype facilitates tumor progression (Silk et al., 2013; Zasadil et al., 2016). Supporting this, patient's outcome correlates with a CIN-high and CIN-low phenotype (Birkbak et al., 2011) since excessive levels of CIN result in a promising prognosis whereas a moderate level of CIN leads to a poor outcome (Roylance et al., 2011).

This might be explained by the fact, that low or moderate CIN leads to adaptability whereas CINhigh phenotype likely causes cell death based on high rates of chromosomes mis-segregation, DNA damage and proteasomal stress (Bakhoum \& Cantley, 2018; Kops, Foltz, \& Cleveland, 2004; Weaver et al., 2007). In case of moderate or low CIN, loss of genes and gene amplifications allow adaptability since specific combinations might represent a general growth advantage or in certain situations like exposure to chemotherapeutics (Thompson, Bakhoum, \& Compton, 2010). This was shown for APC- $\beta$-catenin signaling, which is often inactive due to chromosomal loss and as a result promotes tumor growth (Cahill et al., 1999). Contrary, Santaguida and colleagues revealed that high rates of chromosome mis-segregation resulted in cell cycle arrest and subsequent elimination by the immune system (Santaguida et al., 2017).

\section{Mechanisms of chromosomal instability}

However, the underlying mechanisms causing CIN are not well understood. Several mechanisms are discussed to promote the development of CIN. Among them are an impaired assembly checkpoint (SAC), abnormal sister chromatid cohesion, centrosome amplification and hyper stable kinetochore-microtubule attachments (Thompson et al., 2010). 
The SAC ensures that chromosomes are bioriented before anaphase onset to allow proper chromosome segregation. However, the SAC only senses specific types of erroneous attachments, namely syntelic attachments (Rieder et al., 1995). In general, one can distinguish between four types of kinetochore-microtubules attachments. Proper microtubule-kinetochore attachments are called amphitelic and refer to sister kinetochores attached to microtubules emanating from opposite spindle poles (Fig. 1.11). During bipolar spindle assembly, different types of erroneous kinetochore-microtubule attachments can occur (Fig. 1.11). Syntelic attachments describe the state during which both sister chromatids are bound to microtubules from the same pole. In case of monotelic attachments, only one sister chromatid is bound to microtubules emanating from one spindle pole. Moreover, merotelic attachments refer to the attachment of kinetochores bound to microtubules from opposing poles whereas one sister kinetochore is additionally attached to microtubules emanating from both spindle poles (Cimini, 2007). Lagging chromosomes are the consequence of merotelic attachments and might represent one of the major mechanisms leading to CIN (Bakhoum et al., 2014; Cimini et al., 2001).

In contrast to merotelic attachments, syntelic attachments are recognized by the SAC (Maiato et al., 2004). The latter ensures that chromosomes are properly attached and in case of syntelic attachments, the SAC delays anaphase onset until microtubule-kinetochore attachments are corrected (Musacchio, 2015). As a consequence, a weakened and a hyperactive SAC leads to chromosome mis-segregation and promotes development of CIN and aneuploidy (Sotillo et al., 2007; Yost et al., 2017). However, it was shown that most CIN cells have an intact SAC. Moreover, analysis of DNA sequences from tumor samples revealed no mutations in genes for the SAC (Tighe et al., 2001; Wood et al., 2007).

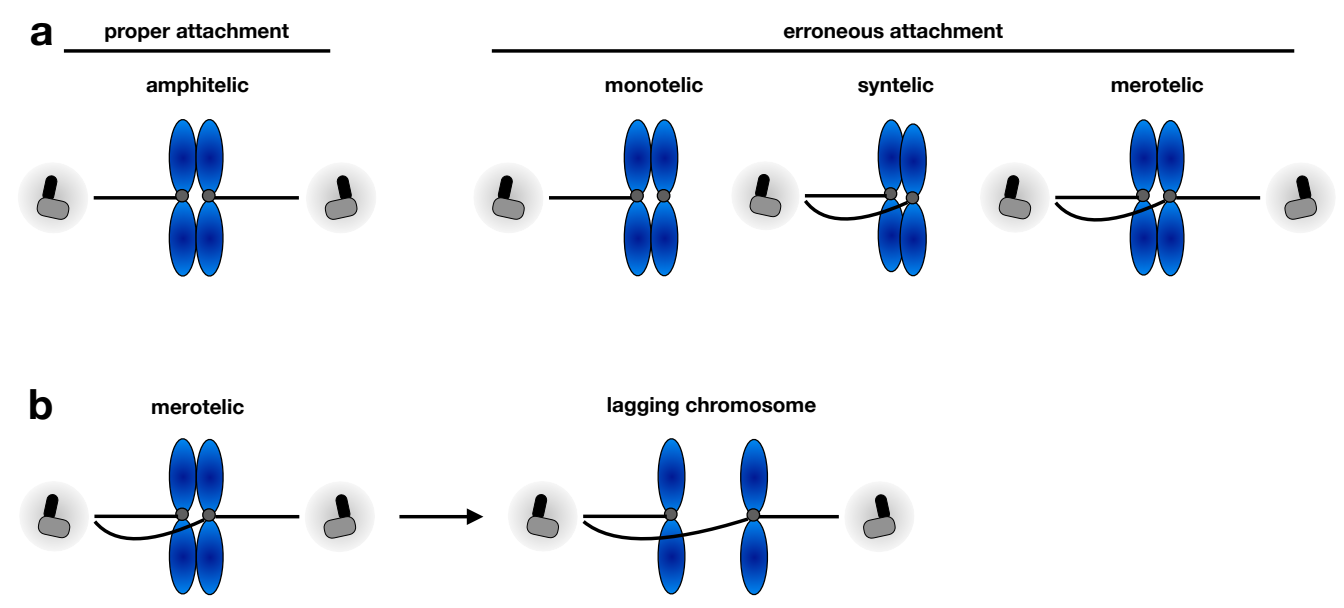

Fig. 1.11 Types of kinetochore-microtubule attachments. 
(a) In case each sister kinetochore is bound to microtubules emanating from opposite poles, a correct amphitelic attachment occurs. The state during which only one kinetochore is bound to microtubules of one pole, is defined as monotelic whereas syntelic refers to sister kinetochores that are bound to microtubules from the same poles. Merotelic attachments define the state in which both sister kinetochores are bound to microtubules from opposing poles whereby one kinetochore is additionally attached to microtubules from both poles. (b) Merotelic attachments promote the generation of lagging chromosomes. Modified from Gregan et al., 2011.

Monotelic attachments are resolved by a correction mechanism involving among others the Ndc80 complex and AuroraB kinase (Cimini, 2007). The latter was shown to localize at the inner centromere where it phosphorylates its substrates like the Ndc80 complex if they're in close proximity to each other. However, in case of proper microtubule-kinetochore attachments, tension between sister kinetochores is generated, and thus, AuroraB is not able to phosphorylate downstream targets. Upon monotelic attachments only low tension is generated thereby allowing AuroraB to phosphorylate $\mathrm{Ndc80}$, which finally results in microtubule turnover, which facilitates the correction of erroneous attachments (Liu et al., 2009). However, in case of merotelic attachments, tension between sister chromatids is generated. When attached in an merotelic manner, chromosomes align tilted at the metaphase plate, which results in close proximity of AuroraB and its targets. Thus, Hec1, a kinetochore protein, is activated and leads to detachment of microtubules and allows MCAK to depolymerize detached microtubules thereby promoting binding of new ones (Cimini, 2007). However, this correction mechanism might not be able to correct all erroneous attachments in case of an increased amount of merotely thereby causing chromosome mis-segregation.

Another mechanism which, plays an important role for faithful chromosome segregation is the cohesin complex, which ensures that sister chromatids are linked to each other until separation in anaphase (Peters et al., 2008). It was shown that cohesion defects are linked to chromosome missegregation and proper cohesion is required for chromosomal stability (Jallepalli et al., 2001). However, it was reported that mutations in genes for cohesion are rarely observed (Barber et al., 2008). Nonetheless, Solomon and colleagues revealed that in bladder cancer a gene encoding for a subunit of the cohesion complex was frequently mutated (Solomon et al., 2013), which was shown to induce aneuploidy (Solomon et al., 2011).

In general, centrosome amplification, which describes the state of an increased number of centrosomes, is associated with $\mathrm{CIN}$ and the frequency of aberrant centrosomes correlates with high-grad and low-grade tumors (D'Assoro, Lingle, \& Salisbury, 2002). In some cases, cells exhibiting supernumery centrosomes form multipolar spindles, which promote chromosome missegregation (Anderhub, Krämer, \& Maier, 2012). Interestingly, Ganem et al., demonstrated that cells with supernumerary centrosomes formed a bipolar mitotic spindle by clustering centrosomes, 
which resulted in viable daughter cells in contrast to multipolar cell divisions. However, an increased amount of lagging chromosomes in anaphase was observed due to merotelic attachments resulting from a transient multipolar spindle (Ganem, Godinho, \& Pellman, 2009).

Lately, another cause for chromosomal instability was described by Ertych et al., who found out that CIN cells exhibited enhanced microtubule plus-end assembly rates during mitosis. Moreover, it was shown that increased microtubule dynamics led to transient spindle geometry defects in prometaphase, which caused merotelic attachments thereby facilitating the generation of lagging chromosomes in anaphase and finally resulted in CIN and aneuploidy (Ertych et al., 2014).

Intriguingly, an EB1 dependent hyperactive TRIO-Rac1-Arp2/3 pathway was shown to be responsible for the generation of lagging chromosomes in CIN cells since long-term inhibition of Rac1, Arp2/3 and TRIO resulted in a stable karyotype of a chromosomally unstable cell line (Berger, 2016). Furthermore, initial experiments revealed a role of TRIO, Rac1 and Arp2/3 for spindle orientation in prometaphase, whereby inhibition of this pathway restored proper spindle orientation in CIN cells (Berger, 2016). Thus, these results support a role of a crosstalk between the actin and microtubule cytoskeleton for mitotic spindle orientation. Importantly, a TRIO-Rac1Arp2/3 pathway downstream of microtubule dynamics has not been implicated in spindle orientation so far and was recently identified by our group (Berger, 2016).

\section{Scope of the study}

Chromosomal instability is a major phenotype of colorectal cancer and is associated with tumor heterogeneity, which allows tumor adaptability and thus, results in therapy resistance and poor prognosis (Gerlinger \& Swanton, 2010; Heng et al., 2013; McGranahan et al., 2012). One of the mechanisms leading to $\mathrm{CIN}$ involves increased microtubule plus-end assembly rates in mitosis (Ertych et al., 2014). This work demonstrated that transient spindle geometry defects based on enhanced microtubule dynamics, promote erroneous kinetochore-microtubule attachments, which cause the generation of lagging chromosomes and finally leads to CIN. Interestingly, spindle geometry defects and spindle misorientation are associated with the development of diseases and especially with cancer (Noatynska, Gotta, \& Meraldi, 2012). Since initial results revealed a role of TRIO, Rac1 and Arp2/3 for spindle orientation, it will be important to further investigate the crosstalk between the actin and microtubule cytoskeleton and in particular, how TRIO, Rac1 and Apr2/3 facilitate spindle misorientation in CIN cells. 


\section{Material and Methods}

\subsection{Material}

Standard consumables as reaction tubes, falcons, pipette tips, filter tips and cell culture dishes were purchased from Greiner BioOne (Frickenhausen, Germany), and ibidi (Martinsried, Germany), Sarstedt (Nümbrecht, Germany) as well as Starlab (Hamburg, Germany).

\subsection{Equipment}

Equipment which was used in this study is listed in Table 2.1.

\section{Table 2.1 Equipment}

\begin{tabular}{|c|c|c|}
\hline Equipment & Model & Company \\
\hline $\mathrm{CO}_{2}$ Incubator & $\begin{array}{l}\text { HERAcell } 240 \mathrm{CO}_{2} \\
\text { Incubator }\end{array}$ & $\begin{array}{l}\text { Thermo Fisher Scientific, Karlsruhe, } \\
\text { Germany }\end{array}$ \\
\hline Centrifuge, cooling & Multifuge X3R & $\begin{array}{l}\text { Thermo Fisher Scientific, Karlsruhe, } \\
\text { Germany }\end{array}$ \\
\hline Centrifuge, tabletop & Biofuge pico & $\begin{array}{l}\text { Thermo Fisher Scientific, Karlsruhe, } \\
\text { Germany }\end{array}$ \\
\hline Centrifuge, tabletop cooling & Biofuge fresco & $\begin{array}{l}\text { Thermo Fisher Scientific, Karlsruhe, } \\
\text { Germany }\end{array}$ \\
\hline Electroporation Device & GenePulser Xcell ${ }^{\odot}$ & $\begin{array}{l}\text { BioRad Laboratories, München, } \\
\text { Germany }\end{array}$ \\
\hline Electrophoresis Power Supply & Power Supply EV231 & Peqlab, Erlangen, Germany \\
\hline Chemiluminescence Imaging & Fusion-SL-3500.WL & Vilber Lourmat, Collégien, France \\
\hline Thermomixer & Thermomixer Comfort $\mathrm{R}$ & Eppendorf, Hamburg, Germany \\
\hline Heating Block & $\begin{array}{l}\text { TDB-120 Dry Block } \\
\text { Thermostat }\end{array}$ & Biosan, Riga, Latvia \\
\hline Laboratory Scale & Sartorius Research R200D & Sartorius, Göttingen, Germany \\
\hline Magnetic Mixer & IKAMAG ${ }^{\odot}$ RCT & IKA Labortechnik, Stauffen, Germany \\
\hline Medical X-Ray Film & Fuji Super RX & $\begin{array}{l}\text { Christiansen und Linhardt, Planegg, } \\
\text { Germany }\end{array}$ \\
\hline \multirow[t]{2}{*}{ Microscope } & Delta Vision Elite ${ }^{\odot}$ & $\begin{array}{l}\text { Applied Precision, Chalfont St. Giles, } \\
\text { UK }\end{array}$ \\
\hline & Leica DMI6000B & Leica, Wetzlar, Germany \\
\hline \multirow[t]{2}{*}{ Microscope Camera } & sCMOS camera & GE Healthcare, Chalfront St. Giles, UK \\
\hline & Leica DFC369 FX & Leica, Wetzlar, Germany \\
\hline
\end{tabular}




\begin{tabular}{|c|c|c|}
\hline Mounting medium & VECTASHIELD ${ }^{\circledR}$ & $\begin{array}{l}\text { Vector Laboratories, Inc., } \\
\text { Peterborough, UK }\end{array}$ \\
\hline Multilabel Reader & Victor $^{\odot} \times 3$ & PerkinElmer, Rodgau, Germany \\
\hline Nitrocellulose Membrane & Protran BA 83 & $\begin{array}{l}\text { GE Healthcare, Chalfront St. Giles, } \\
\text { Great Britain }\end{array}$ \\
\hline Pipettes & Pipetman $^{\odot}$ & $\begin{array}{l}\text { Gilson International, Limburg Offheim, } \\
\text { Germany }\end{array}$ \\
\hline Pipettor & Pipetboy acu & $\begin{array}{l}\text { Integra Biosciences, Fernwald, } \\
\text { Germany }\end{array}$ \\
\hline PVDF Membrane & ImmobilionR-P & Merck Millipore, Darmstadt, Germany \\
\hline Semidry Western Blotting Device & Perfect Blue ${ }^{\odot}$ & Peqlab, Erlangen, Germany \\
\hline Sterile Workbench & HERAsafeM & $\begin{array}{l}\text { Thermo Fisher Scientific, Karlsruhe, } \\
\text { Germany }\end{array}$ \\
\hline Spectrophotometer & NanoDrop 2000 & $\begin{array}{l}\text { Thermo Fisher Scientific, Karlsruhe, } \\
\text { Germany }\end{array}$ \\
\hline Vertical Electrophoresis System & & Own Manufacturing \\
\hline Vortex Mixer & VORTEX-GENIE ${ }^{\odot} 2$ & $\begin{array}{l}\text { Scientific Industries inc., Bohemia, NY, } \\
\text { USA }\end{array}$ \\
\hline \multirow[t]{2}{*}{ Wet Blotting System } & Mini Trans-Blot ${ }^{\circledR}$ Cell & $\begin{array}{l}\text { BioRad Laboratories, München, } \\
\text { Germany }\end{array}$ \\
\hline & & Own Manufacturing \\
\hline X-Ray film processor & Optimax & Protec, Oberstenfeld, Germany \\
\hline
\end{tabular}

\subsubsection{Software}

The software used in this study is listed in Table 2.2

Table 2.2 Software

\section{Software}

Graph Pad Prism 6.0

Fiji Is Just ImageJ

Leica Application Suite 2.7.3.9723

Soft Worx ${ }^{\odot} 6.0$ Software Suite

Soft Worx ${ }^{\odot}$ Explorer 1.3.0

\section{Company}

GraphPad Software, San Diego, California, USA

NIH Image, Bethesda, MD, USA

Leica, Wetzlar, Germany

Applied Precision Inc., Issaquah, WA, USA 


\subsubsection{Chemicals}

All standard chemicals used in this study were purchased from Amersham Biosciences (Buckinghamshire, Great Britain), AppliChem (Darmstadt, Germany), BD Biosciences (Heidelberg, Germany), Carl Roth (Karlsruhe, Germany), Enzo Life Sciences (New York, NY, USA), Fermentas (St. Leon-Roth, Germany), Merck Millipore (Darmstadt, Germany), Promega (Madison, WI, USA), Roche Diagnostics (Mannheim, Germany), Sigma-Aldrich (Taufkirchen, Germany), Thermo Fisher Scientific (Waltham, MA, USA), Th. Geyer (Renningen, Germany) and VWR International (West Chester, PA, USA).

Further chemicals, inhibitors and their function as well as used concentrations are listed in Table 2.3.

Table 2.3 Chemicals

\begin{tabular}{|c|c|c|c|}
\hline Chemical & Concentration & Effect & Company \\
\hline Blebbistatin (+/-) & $25-30 \mu \mathrm{M}$ & $\begin{array}{l}\text { Inhibitory effect on ATPase of } \\
\text { regulatory light chain of NMII }\end{array}$ & $\begin{array}{l}\text { Merck Millipore, } \\
\text { Darmstadt, Germany }\end{array}$ \\
\hline CK666 & $20 \mu \mathrm{M}$ & $\begin{array}{l}\text { Arp } 2 / 3 \text { complex inhibitor, } \\
\text { blocks actin assembly }\end{array}$ & $\begin{array}{l}\text { Merck Millipore, } \\
\text { Darmstadt, Germany }\end{array}$ \\
\hline Dimethylenastron & $2 \mu \mathrm{M}$ & Inhibition of kinesin Eg5 & $\begin{array}{l}\text { Calbiochem, La Jolla, } \\
\text { CA, USA }\end{array}$ \\
\hline ITX3 & $7.5-15 \mu \mathrm{M}$ & $\begin{array}{l}\text { TRIO N-Terminal RhoGEF } \\
\text { Domain Inhibitor }\end{array}$ & $\begin{array}{l}\text { Merck Millipore, } \\
\text { Darmstadt, Germany }\end{array}$ \\
\hline Jasplakinolide & $100-200 \mathrm{nM}$ & Promotes actin polymerization & $\begin{array}{l}\text { Santa Cruz, Dallas, } \\
\text { Texas, USA }\end{array}$ \\
\hline Latrunculin A & $0.75 \mu \mathrm{M}$ & $\begin{array}{l}\text { Inhibition of actin } \\
\text { polymerization }\end{array}$ & $\begin{array}{l}\text { Enzo Life Sciences, New } \\
\text { York, USA }\end{array}$ \\
\hline $\begin{array}{l}\text { Native Clostridium } \\
\text { botulinum Exoenzyme } \\
\text { C3 protein }\end{array}$ & $3 \mu \mathrm{g} / \mathrm{ml}$ & $\begin{array}{l}\text { Inhibition of Rho proteins by } \\
\text { ADP ribosylation }\end{array}$ & $\begin{array}{l}\text { Cytoskeleton Inc., } \\
\text { Denver, USA }\end{array}$ \\
\hline nocodazole & $0.5 \mathrm{nM}$ & $\begin{array}{l}\text { Promotes actin } \\
\text { depolymerization }\end{array}$ & $\begin{array}{l}\text { Sigma-Aldrich } \\
\text { Taufkirchen, Germany }\end{array}$ \\
\hline NSC23766 & $40 \mu \mathrm{M}$ & $\begin{array}{l}\text { Inhibitor of Rac1-GEF } \\
\text { interaction of TRIO and Tiam1 }\end{array}$ & $\begin{array}{l}\text { Santa Cruz, Dallas, } \\
\text { Texas, USA }\end{array}$ \\
\hline $\begin{array}{l}\text { Pertussis toxin from } \\
\text { Bordetella pertussis }\end{array}$ & $100-400 \mathrm{ng} / \mathrm{ml}$ & $\begin{array}{l}\text { Inhibitory effect on the } \alpha- \\
\text { subunit of heterotrimeric G- } \\
\text { proteins }\end{array}$ & $\begin{array}{l}\text { Enzo Life Sciences, New } \\
\text { York, USA }\end{array}$ \\
\hline $\begin{array}{l}\text { Rho Activator I } \\
\text { (Calpeptin) }\end{array}$ & $1 \mu \mathrm{g} / \mathrm{ml}$ & Activation of RhoA, B and C & $\begin{array}{l}\text { Santa Cruz, Dallas, } \\
\text { Texas, USA }\end{array}$ \\
\hline
\end{tabular}




$\begin{array}{lcll}\text { Ro-3306 } & 6 \mu \mathrm{M} & \begin{array}{l}\text { Cdk1 inhibitor, cell cycle } \\ \text { arrest at G2/M-Phase } \\ \text { Formin FH2 Domain inhibitor, } \\ \text { blocks actin nucleation }\end{array} & \begin{array}{l}\text { Santa Cruz, Dallas, } \\ \text { Texas, USA }\end{array} \\ \text { SMIFH2 } & 20 \mu \mathrm{M} & \begin{array}{l}\text { Merck Millipore, } \\ \text { Darmstadt, Germany }\end{array} \\ \text { Taxol } & 0.2-0.5 \mathrm{nM} & \begin{array}{l}\text { Stabilization of microtubules } \\ \text { Sigma-Aldrich }\end{array} \\ \text { Taufkirchen, Germany }\end{array}$

\subsubsection{Primary Antibodies}

In Table 2.4 all primary antibodies and their host species, clonality as well as the used dilution are shown.

Table 2.4 Primary Antibodies

\begin{tabular}{|c|c|c|c|c|c|}
\hline Antigen & Host species & Clonality & Use & Dilution & Company \\
\hline Arp3 (FMS338) & mouse & monoclonal & WB & $1: 500$ & $\begin{array}{l}\text { Sigma-Aldrich, Taufkirchen, } \\
\text { Germany }\end{array}$ \\
\hline$\alpha-T u b u l i n(B-5-1-2)$ & mouse & monoclonal & $\begin{array}{l}\text { IF } \\
\text { WB }\end{array}$ & $\begin{array}{l}1: 700 \\
1: 2000\end{array}$ & $\begin{array}{l}\text { Santa Cruz, Dallas, Texas, } \\
\text { USA }\end{array}$ \\
\hline$\beta$-Actin (A5441) & mouse & monoclonal & WB & $1: 2000$ & $\begin{array}{l}\text { Sigma-Aldrich, Taufkirchen, } \\
\text { Germany }\end{array}$ \\
\hline ch-TOG (H-4) & mouse & monoclonal & WB & $1: 500$ & $\begin{array}{l}\text { Santa Cruz, Dallas, Texas, } \\
\text { USA }\end{array}$ \\
\hline mDia1 (51/mDia1) & mouse & monoclonal & WB & $1: 500$ & $\begin{array}{l}\text { Becton Dickinson, San } \\
\text { Jose, CA, USA }\end{array}$ \\
\hline EB1 (5) & mouse & monoclonal & WB & $1: 500$ & $\begin{array}{l}\text { BD Biosciences, } \\
\text { Heidelberg, Germany }\end{array}$ \\
\hline CENP-C & guinea pig & polyclonal & IF & $1: 1000$ & $\begin{array}{l}\text { MBL International } \\
\text { Corporation, Woburn, USA }\end{array}$ \\
\hline ४-Tubulin (T3559) & rabbit & polyclonal & IF & $1: 700$ & $\begin{array}{l}\text { Sigma-Aldrich, Taufkirchen, } \\
\text { Germany }\end{array}$ \\
\hline §-Tubulin (T6557) & mouse & monoclonal & IF & $1: 1000$ & $\begin{array}{l}\text { Sigma-Aldrich, Taufkirchen, } \\
\text { Germany }\end{array}$ \\
\hline $\begin{array}{l}\text { Stathmin/ } \\
\text { Op18 (E-3) }\end{array}$ & mouse & monoclonal & WB & $1: 300$ & $\begin{array}{l}\text { Santa Cruz, Dallas, Texas, } \\
\text { USA }\end{array}$ \\
\hline
\end{tabular}




$\begin{array}{llllll}\text { Rac1 } & \text { mouse } & \text { monoclonal } & \text { WB } & 1: 500 & \begin{array}{l}\text { Cytoskeleton Inc., Denver, } \\ \text { CO, USA }\end{array} \\ \text { RhoA (26C4) } & \text { mouse } & \text { monoclonal } & \text { WB } & 1: 500 & \begin{array}{l}\text { Santa Cruz, Dallas, Texas, } \\ \text { USA }\end{array} \\ \text { TRIO } & \text { mouse } & \text { polyclonal } & \text { WB } & 1: 500 & \begin{array}{l}\text { Abnova, Taipei City, } \\ \text { Taiwan }\end{array} \\ \text { NAV1 } & \text { mouse } & \text { Monoclonal } & \text { WB } & 1: 500 & \begin{array}{l}\text { Santa Cruz, Dallas, Texas, } \\ \text { USA }\end{array}\end{array}$

\subsubsection{Secondary Antibodies}

In Table 2.5 all secondary antibodies including their species, clonality and used concentration as well as the conjugated molecule.

Table 2.5 Secondary Antibodies

\begin{tabular}{lllllll} 
Antigen & Species & Clonality & Conjungate & Use & Dilution & Company \\
\hline \hline $\begin{array}{l}\text { Anti-Guinea } \\
\text { Pig }\end{array}$ & goat & polyclonal & Alexa-Fluor594 & IF & $1: 1000$ & $\begin{array}{l}\text { Invitrogen, Carlsbad, CA, } \\
\text { USA }\end{array}$ \\
Anti-Mouse & goat & polyclonal & Alexa-Fluor488 & IF & $1: 1000$ & $\begin{array}{l}\text { Invitrogen, Carlsbad, CA, } \\
\text { USA }\end{array}$ \\
Anti-Rabbit & goat & polyclonal & $\begin{array}{l}\text { Alexa-Fluor594 } \\
\text { Invitrogen, Carlsbad, CA, }\end{array}$ & IF & $1: 1000$ & $\begin{array}{l}\text { Ins } \\
\text { USA }\end{array}$ \\
Anti-Mouse & goat & polyclonal & $\begin{array}{l}\text { Horseradish } \\
\text { Peroxidase } \\
\text { (HRP) }\end{array}$ & WB & $1: 10000$ & $\begin{array}{l}\text { Dianova, Hamburg, } \\
\text { Germany }\end{array}$ \\
Anti-Rabbit & goat & polyclonal & $\begin{array}{l}\text { Horseradish } \\
\text { Peroxidase } \\
\text { (HRP) }\end{array}$ & WB & $1: 10000$ & $\begin{array}{l}\text { Dianova, Hamburg, } \\
\text { Germany }\end{array}$
\end{tabular}

\subsubsection{Oligonucleotides}

Table 2.6 provides all used siRNAs, the sequences and their references.

Table 2.6 siRNAs

Target Gene

Arp3
Sequence

5'-AGGUUUAUGGAGCAAGUGA-3'
Reference

Steffen et al. 2006 


\begin{tabular}{|c|c|c|}
\hline & 5'-GCCAAAACCUAUUGAUGUA-3' & \\
\hline CKAP5 & 5'-GAGCCCAGAGTGGTCCAAA-3' & De Luca et al. 2008 \\
\hline DIPAH1 & 5'-GCUGGUCAGAGCCAUGGAU-3' & Arakawa et al. 2003 \\
\hline EB1 & 5'-AUUCCAAGCUAAGCUAGAA-3' & Watson \& Stephens 2006 \\
\hline LUCIFERASE & $5^{\prime}$-CUUACGCUGAGUACUUCGAUU-3' & Elbashir et al. 2001 \\
\hline NAV1 & 5'-AAGAGUUGGCUUCGAAGUUCC-3' & van Haren et al. 2014 \\
\hline Rac1 & 5'-AAGGAGATTGGTGCTGTAAAA-3' & Chan et al. 2005 \\
\hline STATHMIN & 5'-CGUUUGCGAGAGAAGGAUA-3' & Sonego et al. 2013 \\
\hline TRIO & $\begin{array}{l}\text { 5'-GAUAAGAGGUACAGAGAUU-3' } \\
\text { 5'-GGAAGUCGCUCCUUGACAA-3' }\end{array}$ & Cannet et al. 2014 \\
\hline
\end{tabular}

\subsubsection{Plasmids}

Plasmids that were used in this study are listed in Table 2.7 including their purpose and reference.

Table 2.7 Plasmids

\begin{tabular}{|c|c|c|}
\hline Vector & Purpose & Reference \\
\hline pcDNA3.1 & $\begin{array}{l}\text { CMV-promotor driven expression vector } \\
\text { for human cells }\end{array}$ & Invitrogen, Carlsbad, CA, USA \\
\hline pEGFP-C1 & $\begin{array}{l}\text { CMV-promotor driven expression vector } \\
\text { for human cells }\end{array}$ & $\begin{array}{l}\text { Clontech, Saint-Germain-en-Laye, } \\
\text { France }\end{array}$ \\
\hline pEGFP-CKAP5 & $\begin{array}{l}\text { CMV-promotor driven expression of GFP- } \\
\text { tagged } C K A P 5 \text { in human cells }\end{array}$ & $\begin{array}{l}\text { Kindly provided by Prof. Linda } \\
\text { Wordeman (Seattle, WA, USA) }\end{array}$ \\
\hline pEGFP-EB3 & $\begin{array}{l}\text { CMV-promotor driven expression of GFP- } \\
\text { tagged } E B 3 \text { in human cells }\end{array}$ & $\begin{array}{l}\text { Kindly provided by Prof. Linda } \\
\text { Wordeman (Seattle, WA, USA) }\end{array}$ \\
\hline $\begin{array}{l}\text { pEGFP-mCherry- } \\
\text { EB3 }\end{array}$ & $\begin{array}{l}\text { CMV-promotor driven expression of } \\
\text { mCherry- tagged EB3 in human cells }\end{array}$ & $\begin{array}{l}\text { Kindly provided by Prof. Linda } \\
\text { Wordeman (Seattle, WA, USA) }\end{array}$ \\
\hline pEGFP-Rac1 Q61L & $\begin{array}{l}\text { CMV-promotor driven expression of GFP- } \\
\text { tagged constitutively active Rac1 in human } \\
\text { cells }\end{array}$ & $\begin{array}{l}\text { Kindly provided by Prof. Robert } \\
\text { Grosse (Marburg, Germany) }\end{array}$ \\
\hline pEGFP-STATHMIN & $\begin{array}{l}\text { CMV-promotor driven expression of GFP- } \\
\text { tagged STATHMIN }\end{array}$ & $\begin{array}{l}\text { Kindly provided by Gustavo } \\
\text { Baldassarre (Aviano, Italy) }\end{array}$ \\
\hline
\end{tabular}




$\begin{array}{lll}\text { pEGFP-TRIO } & \begin{array}{l}\text { CMV-promotor driven expression of GFP- } \\ \text { tagged TRIO in human cells }\end{array} & \begin{array}{l}\text { Kindly provided by Anne Debant } \\ \text { (Montpellier, France) }\end{array} \\ \text { pEGFP-TRIO-SRNN } & \begin{array}{l}\text { CMV-promotor driven expression of GFP- } \\ \text { tagged TRIO in human cells }\end{array} & \begin{array}{l}\text { Kindly provided by Anne Debant } \\ \text { (Montpellier, France) }\end{array} \\ \text { pXJ-HA-RHOA } & \begin{array}{l}\text { expression of hemagglutinin tagged RhoA } \\ \text { wild type }\end{array} & \begin{array}{l}\text { Kindly provided by Cheng Gee } \\ \text { (Koh, Singapure) }\end{array} \\ \text { pXJ-HA-RHOA- } & \begin{array}{l}\text { expression of hemagglutinin-tagged } \\ \text { constitutively active RhoA }\end{array} & \begin{array}{l}\text { Kindly provided by Cheng Gee } \\ \text { (Koh, Singapure) }\end{array}\end{array}$

\subsubsection{Human Cell Lines}

Table 2.8 lists all human cell lines, their origin and references used in this study.

\section{Table 2.8 Human Cell Lines}

$\begin{array}{lll}\text { Cell line } & \text { Origin } & \text { Reference } \\ \text { DLD-1 } & \text { colon carcinoma } & \text { Obtained from Sigma-Aldrich, Germany } \\ \text { HCT116 cells } & \text { colon carcinoma } & \text { Obtained from ATCC, USA } \\ \text { HT29 } & \text { colon carcinoma } & \text { Obtained from ATCC, USA } \\ \text { LS411N } & \text { colon carcinoma } & \text { Obtained from ATCC, USA } \\ \text { RKO } & \text { colon carcinoma } & \text { Obtained from ATCC, USA } \\ \text { SW620 cells } & \text { colon carcinoma } & \text { Obtained from ATCC, USA } \\ \text { SW480 cells } & \text { colon carcinoma } & \text { Obtained from ATCC, USA } \\ \text { SW837 } & \text { rectum carcinoma } & \text { Obtained from ATCC, USA }\end{array}$

\subsection{Cell Biological Methods}

\subsubsection{Cultivation of Human Cells}

The listed human cell lines were cultured in RPMI1640 (PAN-Biotech GmbH, Aidenbach, Germany) containing $10 \%$ FCS (Gibco by Thermo Fisher, Karlsruhe, Germany) and $1 \%$ penicillin-streptomycin $(100 \mu \mathrm{g} / \mathrm{ml}$ streptomycin, $100 \mathrm{units} / \mathrm{ml}$ penicillin, PAN-Biotech $\mathrm{GmbH}$, Aidenbach, Germany) at $37^{\circ} \mathrm{C}$ and $5 \% \mathrm{CO}_{2}$. Every two to three days cells were passaged. Therefore, cells were washed with 1x PBS and got detached by using Trypsin/EDTA (Lonza Group 
Ltd., Basel, Switzerland). A specific amount of cell suspension was cultured in a new $10 \mathrm{~cm}$ culture dish with fresh culture medium.

Cells were preserved in liquid nitrogen for long-term storage. For this purpose, cells were harvested and resuspended in RPMI1640 with $20 \%$ (v/v) FCS as well as $10 \%$ (v/v) DMSO and gradually cooled down to $-80{ }^{\circ} \mathrm{C}$ with a cryo $1^{\circ} \mathrm{C}$ freezing container. After $24 \mathrm{~h}$, cells were transferred to liquid nitrogen.

\subsubsection{Transfection of human cells}

\subsubsection{SiRNA transfection}

For siRNA transfection either INTERFERin ${ }^{\circledR}$ (Polyplus, Illkirch, France) or ScreenFect ${ }^{\circledR}$ (ScreenFect $\mathrm{GmbH}$, Eggenstein-Leopoldshafen, Germany) were used. For both methods cells were seeded with a confluency of $75 \%$ in 6-well plates and were transfected on the same day.

\section{ScreenFect $\otimes_{\text {siRNA Transfection }}$}

For siRNA transfection with ScreenFect ${ }^{\circledR}$, a master mix was prepared by mixing $30 \mu$ l of dilution buffer with $4 \mu$ of transfection reagent. In a second tube, siRNA was diluted in $30 \mu$ dilution buffer to a final concentration of 60 pmol. The diluted siRNA was mixed with $34 \mu$ of master mix by pipetting 10 times up and down and incubated for $15 \mathrm{~min}$ at RT. In the meantime, cells were washed once with 1x PBS and $1.5 \mathrm{ml} \mathrm{RPMI} 1640$ supplemented with $10 \%$ FCS were added. Afterwards, the transfection mix was added drop-wise onto the cells and incubated overnight at $37{ }^{\circ} \mathrm{C}$ and $5 \% \mathrm{CO}_{2}$. The following day, the medium was changed. Experiments were performed and knockdown was analyzed $48 \mathrm{~h}$ after transfection.

\section{INTERFERin ${ }^{\circ}$ Transfection}

For INTERFERin ${ }^{\circledR}$ transfection, siRNA was diluted in $190 \mu$ l serum free medium to a final concentration of 60 pmol. Subsequently, $6 \mu$ l of INTERFERin ${ }^{\circledR}$ was added, vortexed for $10 \mathrm{sec}$ and incubated for $10 \mathrm{~min}$ at RT. Meanwhile, cells were washed once with 1x PBS and $1 \mathrm{ml}$ fresh cell culture medium was added. The siRNA mix was added drop-wise and cells were incubated for $4 \mathrm{~h}$ at $37{ }^{\circ} \mathrm{C}$ and $5 \% \mathrm{CO}_{2}$. Afterwards, cells were washed once with $1 \mathrm{x}$ PBS and cells were grown over night in fresh cell culture medium. Verification of transfection efficiency and experiments were performed $48 \mathrm{~h}$ after transfection by western blot analysis. 


\subsubsection{Plasmid Transfection}

\section{Electroporation}

For electroporation, cells were harvested and centrifuged for $5 \mathrm{~min}$ at $1500 \mathrm{rpm}$. Afterwards, cells were adjusted to a concentration of $2.5 \times 10^{6} \mathrm{cells} / \mathrm{ml}$ in fresh cell culture medium. $400 \mu \mathrm{l}$ of this suspension was mixed with $10 \mu \mathrm{g}$ of plasmid DNA and transferred to a $4 \mathrm{~mm}$ cuvette. For HCT116 cells, SW620 cells, SW480 cells and RKO, electroporation was performed at $300 \mathrm{~V}$ and $500 \mu \mathrm{F}$. Afterwards, cells were seeded in 6-well plates and medium was changed after $4 \mathrm{~h}$. Experiments were performed $48 \mathrm{~h}$ post transfection while western blot analysis was used to verify transfection efficiency.

\section{Plasmid transfection via Polyethylenimin (PEI)}

For PEI (Sigma Aldrich, Taufkirchen, Germany) transfection, cells were seeded with a confluency of $80 \%$ in 6-well plates and were transfected on the same day. A PEI master mix was prepared by mixing $80 \mu \mathrm{l}$ serum-free medium with $20 \mu \mathrm{IEI}(0.09 \%)$. In a second tube, $20 \mu \mathrm{g}$ of plasmid DNA was mixed with $100 \mu \mathrm{l}$ serum-free medium. To combine, $100 \mu \mathrm{l}$ of PEI master mix was added to $120 \mu$ of diluted plasmid DNA, well mixed and incubated for $15 \mathrm{~min}$ at RT. Meanwhile, cells were washed once with 1x PBS and $1.8 \mathrm{ml}$ serum-free medium was added to the cells. Subsequently, the transfection mix was added drop-wise onto the cells and after $4 \mathrm{~h}$ of incubation, cells were washed four times with 1x PBS and fresh cell culture medium was added. After $48 \mathrm{~h}$, experiments were performed and verification of transfection efficiency was done by western blot analysis.

\section{Plasmid transfection using ScreenFect $\circledast A$}

Cells were seeded with a confluency of $75 \%$ in 6-well plates and transfected on the same day. A master mix, containing $30 \mu \mathrm{l}$ dilution buffer and $6 \mu \mathrm{l}$ ScreenFect ${ }^{\circledR} \mathrm{A}$ (ScreenFect $\mathrm{GmbH}$, Eggenstein-Leopoldshafen, Germany) was prepared. In a second tube, $1.5 \mu \mathrm{g}$ plasmid DNA was diluted in $30 \mu \mathrm{l}$ dilution buffer. To combine, $36 \mu \mathrm{l}$ of master mix was added to the diluted plasmid DNA and well mixed. During 15 min of incubation at RT, cells were washed once with 1x PBS and $1.5 \mathrm{ml}$ fresh cell culture medium without penicillin/streptomycinwas added. Subsequently, the transfection mix was added dropwise onto the cells and incubated overnight. The following day, medium was changed. Verification of transfection efficiency by western blot analysis and experiments were performed $48 \mathrm{~h}$ after transfection. 


\section{Plasmid transfection using Lipofectamine ${ }^{\mathrm{TM}} 3000$ Transfection Reagent}

For Lipofectamin $^{\mathrm{TM}}$ (Invitrogen ${ }^{\mathrm{TM}}$ by Thermo Fisher, Karsruhe, Germany) transfection, cells were seeded with a confluency of $65 \% 16 \mathrm{~h}$ prior to transfection. Two solutions were prepared. On the one hand, a master mix containing $125 \mu \mathrm{l}$ Opti-MEM ${ }^{\mathrm{TM}}$ medium, $5 \mu \mathrm{l}$ of P3000 ${ }^{\mathrm{TM}}$ Reagent and $2.5 \mu \mathrm{g}$ plasmid DNA, was prepared. On the other hand, $125 \mu$ Opti-MEM $^{\text {TM }}$ (Gibco ${ }^{\mathrm{TM}}$ by Thermo Fisher, Karsruhe, Germany) medium was mixed with $6.25 \mu$ l of Lipofectamine ${ }^{\mathrm{TM}} 3000$ Transfection Reagent. To combine, $132 \mu \mathrm{l}$ master mix was resuspended in Lipofectamine ${ }^{\text {TM }}$ mix and the solution was incubated for $15 \mathrm{~min}$ at RT. In the meantime, cells were washed once with 1x PBS and $2 \mathrm{ml}$ fresh medium without penicillin/streptomycinwas added. Subsequently, the transfection mix was added dropwise onto the cells for at least $4 \mathrm{~h}$ or overnight. Experiments were performed $48 \mathrm{~h}$ post transfection while western blot analysis was used to verify transfection efficiency.

\subsubsection{Synchronization of human cells}

\section{Synchronization of cells via a double thymidine block}

To arrest cells at G1/S-Phase and accumulate cells in anaphase, cells were grown for $16 \mathrm{~h}$ in cell culture medium supplemented with $2 \mathrm{mM}$ thymidine. The following day, cells were washed 6 times for $5 \mathrm{~min}$ with cell culture medium. After releasing the cells into fresh cell culture medium for $7.5 \mathrm{~h}$, cells were seeded onto glass coverslips and again cultured in $2 \mathrm{mM}$ thymidine for $16 \mathrm{~h}$. The following day, cells were washed and released as described above. To analyze lagging chromosomes in anaphase cells were fixed after $8.5 \mathrm{~h}$ to accumulate cells at anaphase.

\section{Synchronization of cells by Cdk1 inhibition}

To arrest cells at $\mathrm{G}_{2} / \mathrm{M}$-Phase, cells were seeded onto fibronectin coated coverslips (Neuvitro Corporation, Vancouver, USA) and grown over night in cell culture medium. The following day, cell culture medium supplemented with $6 \mu \mathrm{M}$ Cdk1 inhibitor (Ro 3306) was added. After $4 \mathrm{~h}$ of incubation, cells were washed five times with pre-warmed medium and were fixed after releasing the cells into fresh cell culture medium for $10 \mathrm{~min}$.

\subsubsection{Immunofluorescence microscopy}

For immunofluorescence microscopy experiments, cells were fixed with $2 \%$ PFA for 5 min at RT and subsequently for 5 min with ice-cold $100 \%$ methanol at $-20{ }^{\circ} \mathrm{C}$. Afterwards, cells were washed with 1x PBS and blocked with $5 \%$ FCS in PBS for 20 min Subsequently, antibodies for a-tubulin (1:700) and CENP-C (1:1000) were prepared in $2 \%$ FCS/PBS and cells were incubated for 90 min at RT to stain microtubules and centromeres, respectively. Afterwards, cells were washed 
three times with 1x PBS and incubated with the secondary, fluorescence-labeled antibody (1:1000 in $2 \%$ FCS/PBS) for $90 \mathrm{~min}$ at RT. Directly following, cells were incubated for 5 min with Hoechst33342, which was diluted 1:15 000 in 1x PBS. Afterwards, cells were washed four times with 1x PBS and once with water. After the cells were air-dried, coverslips were mounted using VectaShield $^{\circledR}$ (Vector Laboratories, Burlingame, CA, USA).

To analyze fixed cells a Leica DM600B fluorescence microscope and an ORCA-ER camera was used. Images were acquired with an optical z-spacing of $0.4 \mu \mathrm{m}$ using a $60 x$ magnification. For further image processing, the Leica LAS-AF software was used.

\subsubsection{Analysis of spindle axis alignment in prophase, prometa- and metaphase}

To determine the angle of spindle axis alignment of cells in prometaphase and metaphase, cells were grown asynchronously overnight on fibronectin coated coverslips. For accumulation of cells in prophase, cells were arrested at $\mathrm{G}_{2} / \mathrm{M}$-Phase by using the Cdk1 inhibitor Ro 3306 . Before fixation, cells were washed once with 1x PBS and fixed with ice cold $100 \% \mathrm{MeOH}$ for 6 min at $20{ }^{\circ} \mathrm{C}$. Afterwards, cells were washed with 1x PBS and blocked with $10 \%$ FCS in 1x PBS for $20 \mathrm{~min}$ at RT. To visualize centrosomes and microtubules, cells were stained for $\gamma$-tubulin (1:1000 in $2 \%$ FCS/PBS) and $\alpha$-tubulin (1:700 in $2 \%$ FCS/PBS) for 90 min at RT. After washing the cells three times with 1x PBS, cells were incubated for $90 \mathrm{~min}$ at RT with fluorescence labeled secondary antibodies (1:1000 in $2 \%$ FCS/PBS). Subsequently, cells were incubated for 5 min with Hoechst33342 (1:15 000 in PBS) at RT for DNA staining. Afterwards, cells were washed four times with 1x PBS and once with water. Air-dried coverslips were mounted using VectaShield.

Microscopy of fixed and stained cells was performed on a Delta Vision Elite ${ }^{\odot}$ microscope equipped with a PCO Edge sCMOS camera. Images were acquired using an Olympus 60x 1.40 NA objective and the softWoRx ${ }^{\circledR} 6.0$ Software Suite. Images were taken with an optical z-stack spacing of $0.4 \mu \mathrm{m}$ and deconvolved. Further analysis was performed using the softWoRx ${ }^{\circledR}$ Explorer 1.3.0.

To calculate the angle of spindle axis alignment, the following formula was used:

$$
\alpha(\text { spindle axis angle })=\frac{\text { number of } z \text {-stacks } * 0.4}{\text { distance between centrosomes }} * \frac{180}{\pi}
$$




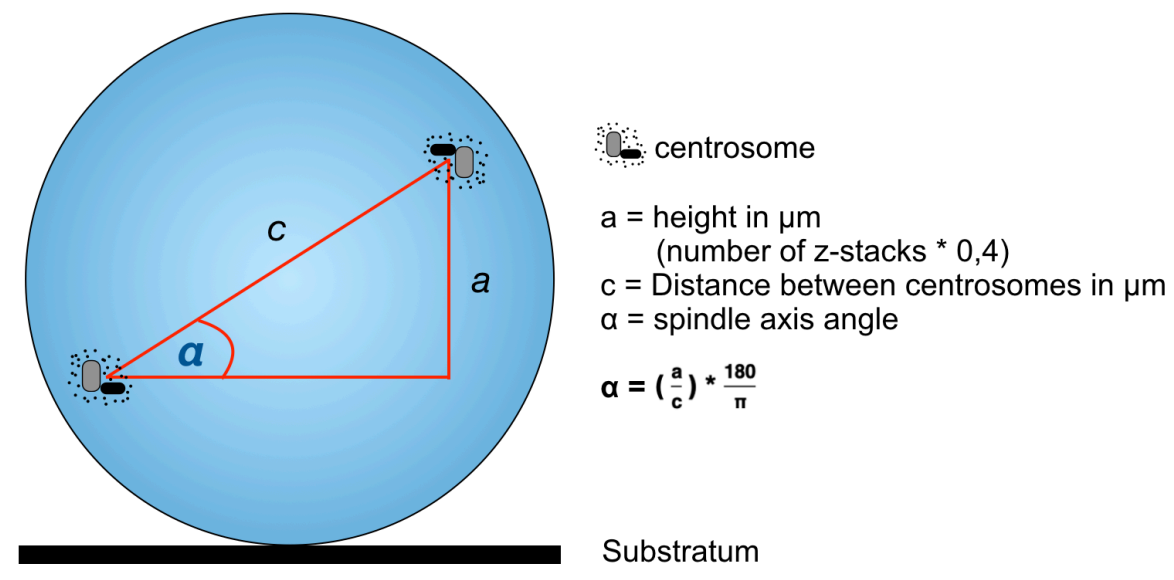

Fig. 2.1 Illustration for calculating the angle of spindle axis alignment.

\subsubsection{Analysis of microtubule plus-end assembly rates}

To analyze microtubule plus-end assembly rates cells were transfected via electroporation with $10 \mu \mathrm{g}$ pEGFP-EB3 plasmid DNA. The following day, cells were seeded in a $35 \mathrm{~mm}$ imaging dish or a $\mu$-Slide 8-Well and grown overnight. After $48 \mathrm{~h}$ of transfection, cells were washed once with 1x PBS and $1.5 \mathrm{ml}$ RPMI1640 without phenol red and supplemented with $10 \%$ FCS was added to the cells. In order to arrest cells in mitosis, cells were treated with $2 \mu \mathrm{M}$ DME for 60 - 90 min Measurement of microtubule plus-end assembly rates was performed at $37^{\circ} \mathrm{C}$ and $5 \% \mathrm{CO}_{2}$ using a DeltaVision-ELITE microscope and a PCO Edge sCMOS camera. Images were acquired every two seconds for 30 seconds in total with an optical z-stack spacing of $0.4 \mu \mathrm{m}$. Afterwards, images were deconvolved and analyzed using the softWoRx ${ }^{\circledR}$ Explorer 1.3.0.

To calculate microtubule plus-end assembly rates, the growth of one plus tip between two frames was measured. For statistical analysis, 20 microtubules of one cell were measured and for one independent experiment 10 cells were analyzed.

\subsection{Statistics}

For all graphs, an unpaired t-test was performed with the indicated p-values as: ${ }^{* * * *}: p \leq 0.0001$; ${ }^{* * *}: p \leq 0.001,{ }^{* *}: p \leq 0.01,{ }^{*}: p \leq 0.05$, ns (not significant): $p>0.05$ 


\subsection{Protein biochemistry}

\subsubsection{Preparation of Protein Lysates}

Cells were washed once with $1 \mathrm{x}$ PBS and harvested by using $0.5 \mathrm{mM}$ PBS/EDTA. After a centrifugation at $2000 \mathrm{rpm}$ for $5 \mathrm{~min}$ the supernatant was removed and $50-70 \mu$ of lysis buffer was added (50 mM Tis-HCl pH 7.4, 150 mM NaCl, 5 mM EDTA, 5 mM EGTA, 20 mM Na3Vo4, 25 mM $\beta$-Glycerophosphate, $50 \mathrm{mM} \mathrm{NaF}, 5 \mathrm{mM} \mathrm{Na} 2 \mathrm{MoO} 4,1 \%$ (v/v) Igepal ${ }^{\odot}$, $0.1 \%$ (w/v) SDS, $0.1 \%$ Na-Deoxycholate). Following a $10 \mathrm{~min}$ incubation on ice, lysates were centrifugated at $14.8000 \mathrm{rpm}$ for $10 \mathrm{~min}$ at $4{ }^{\circ} \mathrm{C}$. Afterwards, the supernatant was removed, and the lysate was transferred to a new tube and immediately used for protein determination or until further use stored at $-20{ }^{\circ} \mathrm{C}$.

\subsubsection{Protein Determination}

The concentration of protein lysates was measured by using the Bio-Rad $D C^{\mathrm{TM}}$ Protein Assay (BioRad, Hercules, CA, USA) according to the manufacturer's instructions. A VICTOR ${ }^{\odot} \mathrm{X} 3$ microplate reader was used for photometric measurements. Afterwards, 50 or $100 \mu \mathrm{g}$ of cell lysate were transferred into a new tube and 5x SDS buffer (50\% glycerol, $15 \%$ (v/v) $\beta$-mercaptoethanol, $15 \%(\mathrm{w} / \mathrm{v})$ SDS, $0.25 \%(\mathrm{w} / \mathrm{v})$ bromophenol blue) was added. After a $5 \mathrm{~min}$ incubation at $95{ }^{\circ} \mathrm{C}$ to denature proteins, samples were stored at $-20{ }^{\circ} \mathrm{C}$ until further use.

\subsubsection{Sodium Dodecylsulfate Polyacrylamide Gel Electrophoresis (SDS-PAGE)}

For separation of proteins, a discontinuous SDS-PAGE was used. The SDS gel consists of a $5 \%$ stacking gel (300 nM TRIS-HCl pH 6.8, $0.1 \%$ (w/v) SDS, $5 \%$ (v/v) Rotiphorese Gel 30॰) and dependent on the molecular weight, a resolving gel from $6 \%$ up to $13 \%$ (500 nM TRIS-HCI $\mathrm{pH} 8.8,0.1 \%(\mathrm{w} / \mathrm{v})$ SDS, 6-13 \% (v/v) Rotiphorese Gel 30 ${ }^{\circ}$ ) was used. Prepared lysates were loaded onto the gel and $5 \mu \mathrm{l}$ of PageRuler Prestained Protein Ladder (Fermentas, St. Leon-Rot, Germany) was used. Separation of proteins was carried out for $1 \mathrm{~h}$ at $28 \mathrm{~mA}$ and 2 to $3 \mathrm{~h}$ at $35 \mathrm{~mA}$ using an SDS running buffer (25 mM TRIS-HCl pH 6.8, $192 \mathrm{mM}$ glycine, $0.15 \%$ (w/v) SDS).

\subsubsection{Western Blot}

\section{Semi-dry-blotting}

For semi-dry blotting the gel was mounted on a nitrocellulose membrane and blotting was performed for $1.5 \mathrm{~h}$ at $200 \mathrm{~mA}$ with an appropriate amount of transfer buffer $(24.8 \mathrm{mM}$ TRIS-HCl $\mathrm{pH}$ 8.0, $170 \mathrm{mM}(\mathrm{v} / \mathrm{v})$ glycine, $0.0025 \%$ (w/v) SDS, $20 \%$ methanol). 


\section{Wet-blotting}

For proteins larger than $100 \mathrm{kDa}$ a wet-blotting procedure was performed. Dependent on the molecular weight of the protein of interest either a nitrocellulose or a PVDF (protein transfer and immobilization on a polyvinylidene fluoride) membrane was used. In the latter case, the PVDF membrane was activated by a short incubation in $100 \% \mathrm{MeOH}$ and washed several times with distilled water. Proteins were transferred in a wet-blot chamber for $3 \mathrm{~h}$ at $450 \mathrm{mM}$ with an appropriate amount of transfer buffer $(24.8 \mathrm{mM}$ TRIS-HCl pH 8.0, $170 \mathrm{mM}(\mathrm{v} / \mathrm{v})$ glycine, $0.0025 \%$ (w/v) SDS, $13 \%$ methanol)

\section{Detection of proteins by chemiluminescence}

Following the protein transfer, membranes were blocked with $5 \%$ milk powder in TBS (50mM TRIS-HCl pH 7.2, $160 \mathrm{mM} \mathrm{NaCl}$ ) for $30 \mathrm{~min}$ at RT. To detect the protein of interest, a primary antibody was diluted in $3 \% \mathrm{BSA} / \mathrm{TBS}$ and membranes were incubated at $4{ }^{\circ} \mathrm{C}$ overnight shaking. The next day, membranes were washed 3 times for 10 min each with TBS-T (TBS with $0.1 \%$ Tween). Subsequently, membranes were incubated with a horseradish peroxidase (HRP) conjugated secondary antibody for $60 \mathrm{~min}$ at RT. Afterwards, membranes were washed $3-6$ times each for 10 min with TBS-T.

Proteins were detected by using chemiluminescence. Therefore, membranes were incubated for $30 \mathrm{sec}$ in an enhanced chemiluminescence $(E C L) \operatorname{mix}(0.1 \mathrm{mM}$ TRIS-HCl pH 8.5, $2.5 \mathrm{mM}$ luminol, $0.4 \mathrm{mM} \beta$-coumaric acid, $0.03 \% \quad \mathrm{H}_{2} \mathrm{O}_{2}$ ). Chemiluminescence was detected by using a chemiluminescence imaging device or by medical X-ray films in combination with an X-ray film processor.

\subsection{Molecular Biological Methods}

\subsubsection{Escherichia coli (E. coli) cells}

The E. coli strain $\mathrm{DH} \mathrm{aF}^{-} \phi 80$ lacZ $\Delta \mathrm{M} 15 \Delta\left(\right.$ lacZYA-argF) U169 deoR recA1 $h s d R 17\left(\mathrm{r}_{\mathrm{k}}^{-}, \mathrm{m}_{\mathrm{k}}^{+}\right)$phoA supE44 thi-1 gyrA96 relA1 $\lambda^{-}$was used.

\subsubsection{Cultivation of $E$. coli}

E. coli were cultivated in Luria Bertani medium (LB medium) at $37^{\circ} \mathrm{C}$ in an incubation shaker at $110 \mathrm{rpm}$. To select transformed bacteria either $100 \mathrm{mg} / \mathrm{ml}$ ampicillin or $50 \mathrm{mg} / \mathrm{l} \mathrm{kanamycin} \mathrm{were}$ added. 


\subsubsection{Generation of competent $E$. coli cells}

For the generation of chemically competent $E$. coli cells, $5 \mathrm{ml}$ LB medium were inoculated with $E$. coli $\mathrm{DH} 5 \alpha$. After the cells were grown overnight, shaking at $37^{\circ} \mathrm{C}$, the culture was transferred to $400 \mathrm{ml}$ LB medium. After reaching a density of $O D_{600}=0.5$ the cells were incubated for 5 min on ice and centrifuged for $5 \mathrm{~min}$ at $2000 \mathrm{rpm}$. After removing the supernatant, cells were resuspended in $40 \mathrm{ml}$ of Tfbl buffer (30 mM potassium acetate, $100 \mathrm{mM} \mathrm{RbCl}, 10 \mathrm{mM} \mathrm{CaCl}, 15 \%$ glycerol, $\mathrm{pH}$ 6.0) and incubated on ice for 5 min Subsequently, cells were centrifuged at $2000 \mathrm{rpm}$ for $5 \mathrm{~min}$ and resuspended in $4 \mathrm{ml}$ Tfbll buffer (10 mM MOPS, $75 \mathrm{mM} \mathrm{CaCl}, 10 \mathrm{mM} \mathrm{RbCl}, 15 \%$ glycerol, $\mathrm{pH}$ 6.5) followed by an incubation for $15 \mathrm{~min}$ on ice. Aliquots of $100 \mu$ were snap frozen in liquid nitrogen and stored at $-80^{\circ} \mathrm{C}$.

\subsubsection{Transformation of $E$. coli cells}

For transformation of $E$. coli DH5a, $50 \mu$ l of competent cells were thawed on ice and 1-2 $\mu \mathrm{g}$ plasmid DNA was added and gently mixed. After an incubation of 20 min on ice, a heat shock was performed at $42{ }^{\circ} \mathrm{C}$ for $60 \mathrm{sec}$ and $600 \mu \mathrm{l}$ of LB medium without selective antibiotics was added. Following an incubation shaking on a thermomixer for $45 \mathrm{~min}$ at $37^{\circ} \mathrm{C}$, the cell suspension was transferred to $400 \mathrm{ml} \mathrm{LB}$ medium containing the appropriate antibiotic to select transformed bacteria. After an incubation shaking overnight at $37^{\circ} \mathrm{C}$, cells were harvested and centrifuged for $20 \mathrm{~min}$ at $4{ }^{\circ} \mathrm{C}$ and $4000 \mathrm{rpm}$. Afterwards, the remaining LB was removed and the pellet was stored at $-20^{\circ} \mathrm{C}$ until further use.

\subsubsection{Plasmid Isolation}

Plasmid isolation was carried out by using the NucleoBond ${ }^{\odot}$ PC 100 X-TRA Midi Kit (MACHEREYNAGEL GmbH \& Co. KG, Düren, Germany) according to the manufacturer's specification. By using the NanoDrop ${ }^{\odot} 2000$ spectrophotometer the amount of plasmid DNA was measured. 


\section{Results}

\subsection{Abnormal spindle structures of CIN cells are associated with a transient spindle axis misalignment in prometaphase}

Recent work by our group revealed that CIN cells exhibit a higher proportion of cells with lagging chromosomes in comparison to chromosomally stable cells, due to enhanced microtubule plusend assembly rates during mitosis (Ertych et al., 2014). Initially, a panel of colorectal cancer cells was used to systematically analyze the occurrence of lagging chromosomes (Fig. 3.1). The used panel of CRC included three chromosomally stable cell lines (HCT116 cells, RKO and DLD-1), which are microsatellite unstable (MIN/MSI) and five chromosomally unstable cell lines (SW480 cells, SW620 cells, SW837, HT29 and LS411N). To accumulate cells in anaphase, cells were synchronized via a double thymidine block and stained for $\alpha$-tubulin to visualize microtubules and CENP-C to detect centromeres. A lagging chromosome was defined as a CENP-C positive chromatid, which lagged behind during chromosome segregation in anaphase (Fig. 3.1b). Importantly, only $1.5 \%$ of MIN/MSI cells exhibited lagging chromosomes whereas $6.1 \%$ of CIN cells showed lagging chromosomes in anaphase (Fig. 3.1a). Thus, the proportion of CIN cells with lagging chromosomes in anaphase is increased in comparison to chromosomally stable MIN/MSI cells, which was reported before und thus, could be verified (Ertych et al., 2014).
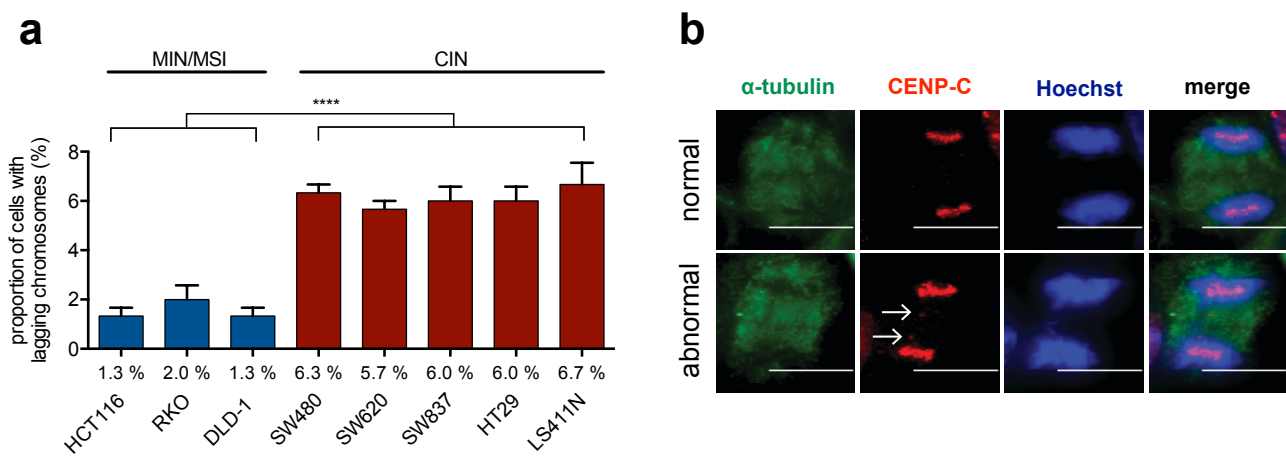

Fig. 3.1 CIN cells exhibit a higher proportion of cells with lagging chromosomes in anaphase.

(a) Analysis of lagging chromosomes in MIN/MSI and CIN CRC during anaphase. Cells were synchronized at $\mathrm{G}_{1} / \mathrm{S}$-phase transition by a double thymidine block and fixed after releasing the cells for $8.5 \mathrm{~h}$. Cells were stained for immunofluorescence microscopy to analyze lagging chromosomes. Bar graphs show the proportion of $\alpha$-tubulin and CENP-C. Cells exhibiting a colocalization of a lagging chromatid colocalized with CENP-C indicates the occurrence of a lagging chromosome $(n=3$, total amount of 300 analyzed anaphases, mean values \pm SEM, $t$-test, ${ }^{* * * *}: p \leq 0.0001$ ). (b) Representative immunofluorescence image of an anaphase without a lagging chromosome (normal) and the occurrence of a lagging chromosome (abnormal). Cells were stained for $\alpha$-tubulin, CENP-C and Hoechst33342. Scale : $5 \mu \mathrm{m}$. 
Since Ertych et al. showed that spindle geometry defects led to lagging chromosomes, mitotic spindles were further investigated including determination of the angle of spindle axis alignment during prometaphase and analysis of the spindle structure. For this purpose, cells were seeded on fibronectin coated coverslips and were grown overnight.
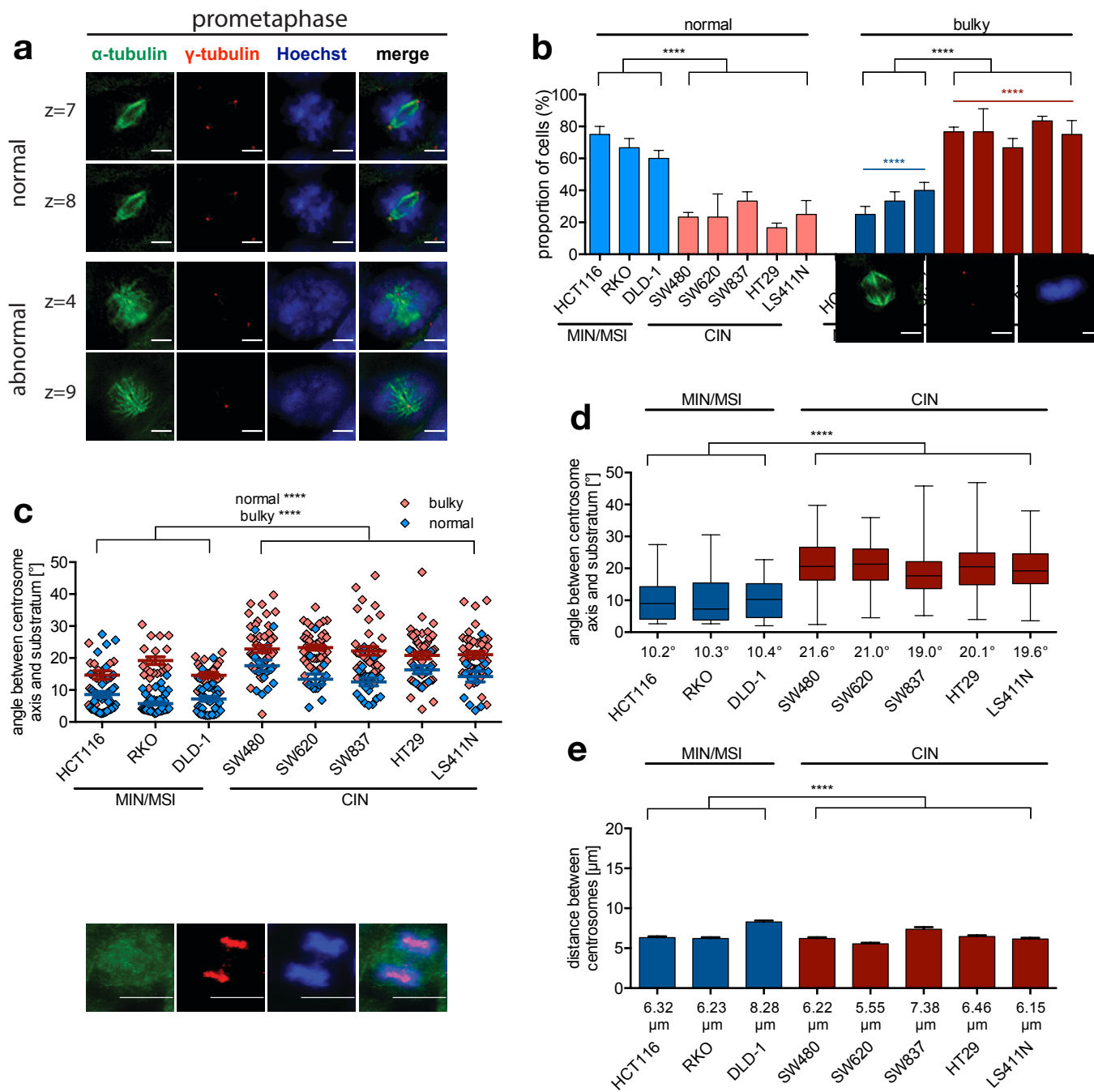

Fig. 3.2 Altered spindle structures of CIN cells correlate with an increased angle of spindle axis alignment in prometaphase.

(a) Representative immunofluorescence images of a cell in prometaphase stained for $\alpha$-tubulin and $y$ tubulin. $Z$ relates to the $z$-stack in which the centrosome is in focus. Scale: $5 \mu \mathrm{m}$. (b) Proportion of cells that have a normal or bulky spindle structure in prometaphase. Graph bar show mean values \pm SEM. ( $n=3,60$ cells analyzed in total, $t$-test). ${ }^{* * * *}$ indicates normal vs. bulky in MIN/MSI cells while ${ }^{* * * *}$ indicates normal vs. bulky in CIN cells. (c) Measurement of spindle axis alignment in prometaphase of cells shown in (b) in relation to their spindle structure. Cells were grown on fibronectin coverslips and stained for $\alpha$-tubulin and $\mathrm{y}$-tubulin for immunofluorescence microscopy. The box and whisker diagram show the median (mean values are indicated), the $25^{\text {th }}$ to $75^{\text {th }}$ percentile and the minimum and maximum values (d) Spindle axis alignment of cells shown in (c) without categorizing. (e) Bar graphs illustrate the distance between centrosomes of analyzed cells shown in (c). ${ }^{* * * *}: p \leq 0.0001$. 
While analyzing spindle structures of prometaphase cells it was observed that CIN cells exhibited abnormal spindle structures in comparison to MIN/MSI cells (Fig. 3.a). Since $76 \%$ of CIN cells showed bulky structured spindles, this phenotype was more pronounced in CIN cells than in MIN/MSI cells, of which only $24 \%$ exhibited abnormal spindle structures (Fig. 3.2b). Interestingly, these structural differences correlated with an increased angle of spindle axis alignment in prometaphase in CIN cells (Fig. 3.2c). Normal shaped MIN/MSI and CIN cells revealed an angle of $7^{\circ}$ and $15^{\circ}$, respectively whereas bulky structured spindles exhibited an angle of $16^{\circ}$ in MIN cells and $22^{\circ}$ in CIN cells (Fig. 3.2c). Moreover, when MIN/MSI and CIN cells were analyzed regarding their spindle angle without categorizing, MIN/MSI cells showed an average angle of $10.3^{\circ}$ whereas the angle of spindle axis alignment in CIN cells was significantly increased to an average of $20.3^{\circ}$ (Fig. 3.2d). Since the distance between centrosomes is a crucial parameter for calculating the angle of spindle axis alignment, this factor was also analyzed (Fig. 3.2e). In general, a greater distance between centrosomes results more likely in a smaller angle of spindle axis alignment whereas shorter distances lead to greater angle of spindle axis alignment. No difference regarding the distance between centrosome was observed since MIN/MSI cells showed an average distance of $6.9 \mu \mathrm{m}$ while CIN cells exhibited an average distance of $6.3 \mu \mathrm{m}$ (Fig. 3.2e). To investigate whether spindle axis misalignment in CIN cells is characteristic throughout mitosis or whether it is only a transient phenotype, cells in prophase and in metaphase were analyzed. In prophase, no structural differences of microtubules arising from centrosomes was observed in prophase (Fig. 3.3a). Moreover, there was only little difference of the average spindle axis alignment angle between MIN/MSI and CIN cells (Fig. 3.3b). Even though the spindle angle was almost doubled in CIN cells during prometaphase, there was no difference regarding spindle angle and structures between MIN/MSI and CIN cells in metaphase (Fig. 3.3d). Furthermore, the distance between centrosomes did not vary between CIN and MIN/MSI cells (Fig. 3.3c,f).

These results indicate a strong correlation between aberrant spindle structures, a transient spindle axis misalignment in prometaphase and an increased number of cells exhibiting lagging chromosomes in CIN cells. In contrast, MIN/MSI cells showed proper spindle axis alignment in prometaphase and a reduced proportion of cells with lagging chromosomes. 

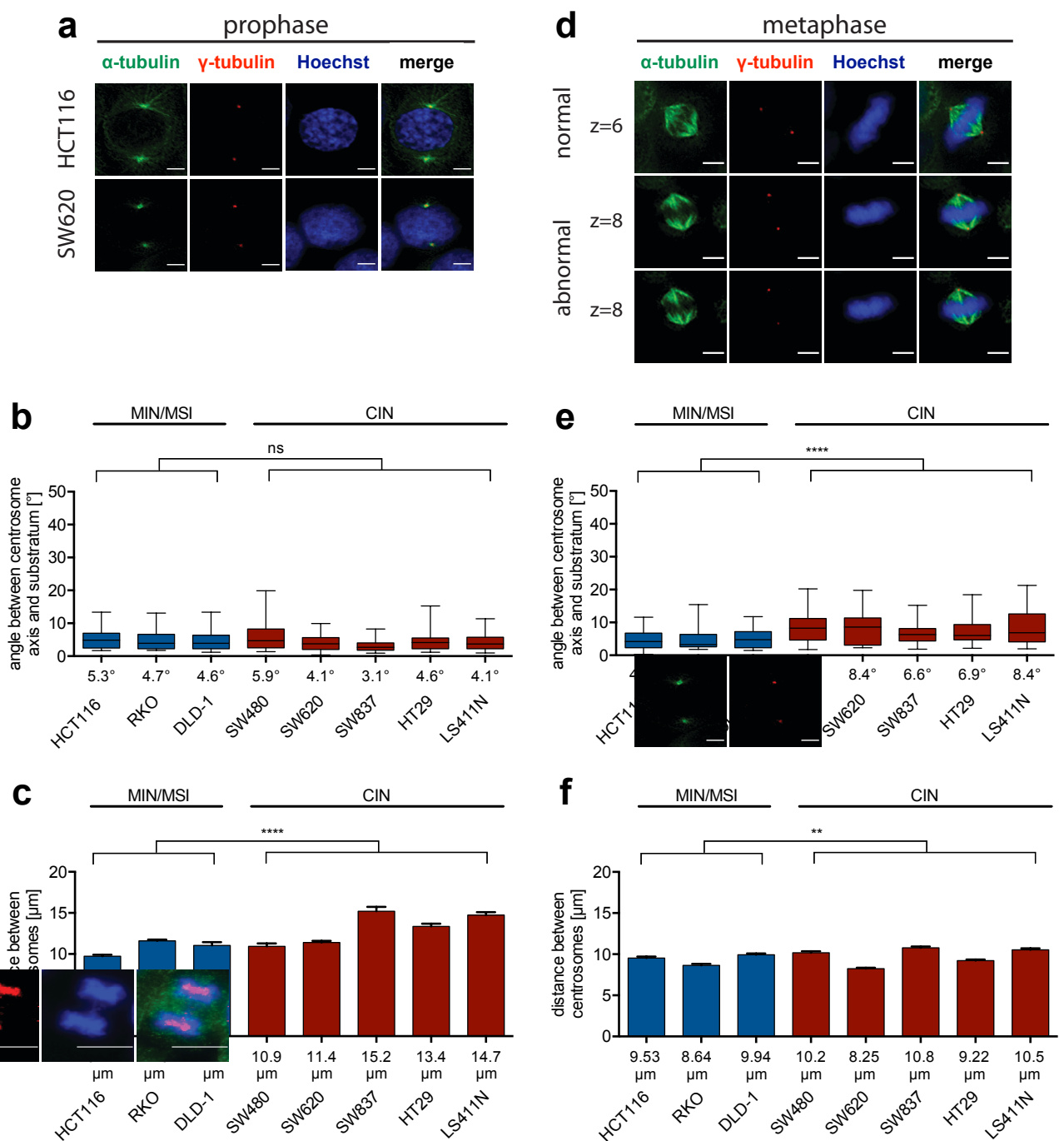

Fig. 3.3 Spindle axis misalignment in CIN cells is present prometaphase but not in prophase and metaphase.

(a,d) Representative immunofluorescence images of a cell in prophase (a), and metaphase (d) stained for a-tubulin, $y$-tubulin and Hoechst33342. Z relates to the z-stack in which the centrosome is in focus. Scale : $5 \mu \mathrm{m}$. (b, e) Measurement of the angle between the centrosome axis and substratum of CIN and MIN/MSI cells in prophase (a) and metaphase (d). For each approach cells were seeded on fibronectin coated coverslips and were analyzed. For immunofluorescence microscopy, cells were fixed, stained for a-tubulin and $\mathrm{\gamma}$-tubulin and analyzed. To accumulate cells in prophase, Cdk1 was inhibited by using $6 \mu \mathrm{M}$ Ro-3306 for $4 \mathrm{~h}$ to synchronize cells at $\mathrm{G}_{2} / \mathrm{M}$-phase. After releasing the cells for $10 \mathrm{~min}$ into mitosis, cells were fixed. The box and whisker diagram show the median (mean values are indicated), the $25^{\text {th }}$ to $75^{\text {th }}$ percentile and the minimum and maximum values $(n=3,60$ cells analyzed in total, $t$-test). (c,f) Bar graphs illustrate the distance between two centrosomes of analyzed cells showed in $(a-b){ }^{* * * *}: p \leq 0.0001,{ }^{* *}: p \leq 0.01$, ns $=$ not significant; $p>0.5$ 


\subsection{Enhanced microtubule plus-end assembly rates cause spindle axis misalignment in prometaphase}

Since it was observed that spindle axis misalignment is a major phenotype in CIN cells, it was of interest to further analyze the role of microtubule plus-end dynamics in the context of spindle axis alignment. For this purpose, microtubules plus-end assembly rates were modulated, and spindle axis alignment and lagging chromosomes were analyzed. To measure microtubule plus-end assembly rates, GFP-tagged EB3 was expressed as this allows tracking of growing microtubules (Stepanova et al., 2003). Moreover, cells were arrested in mitosis by using DME (dimethylenastron), which leads to Eg5 inhibition (Gartner et al., 2005). The following different approaches were used to influence microtubule plus-end assembly rates: Taxol as a wellestablished microtubule-stabilizing agent, is known to decrease microtubule dynamics (Jordan \& Wilson, 2004). The microtubule polymeriase ch-TOG (CKAP5) represents another candidate that has an impact on microtubule dynamics, since it was shown to promote microtubules growth by binding to microtubules and adding tubulin subunits (Brouhard et al., 2008). Also, stathmin/Op18 is a regulator of microtubules dynamics. By sequestering free tubulin, it was demonstrated that stathmin destabilized microtubules (Lawler, 2004). Thus, in the following experiments microtubule dynamics were modulated by downregulation of CKAP5 as well as STATHMIN or by addition of low doses of Taxol.

a
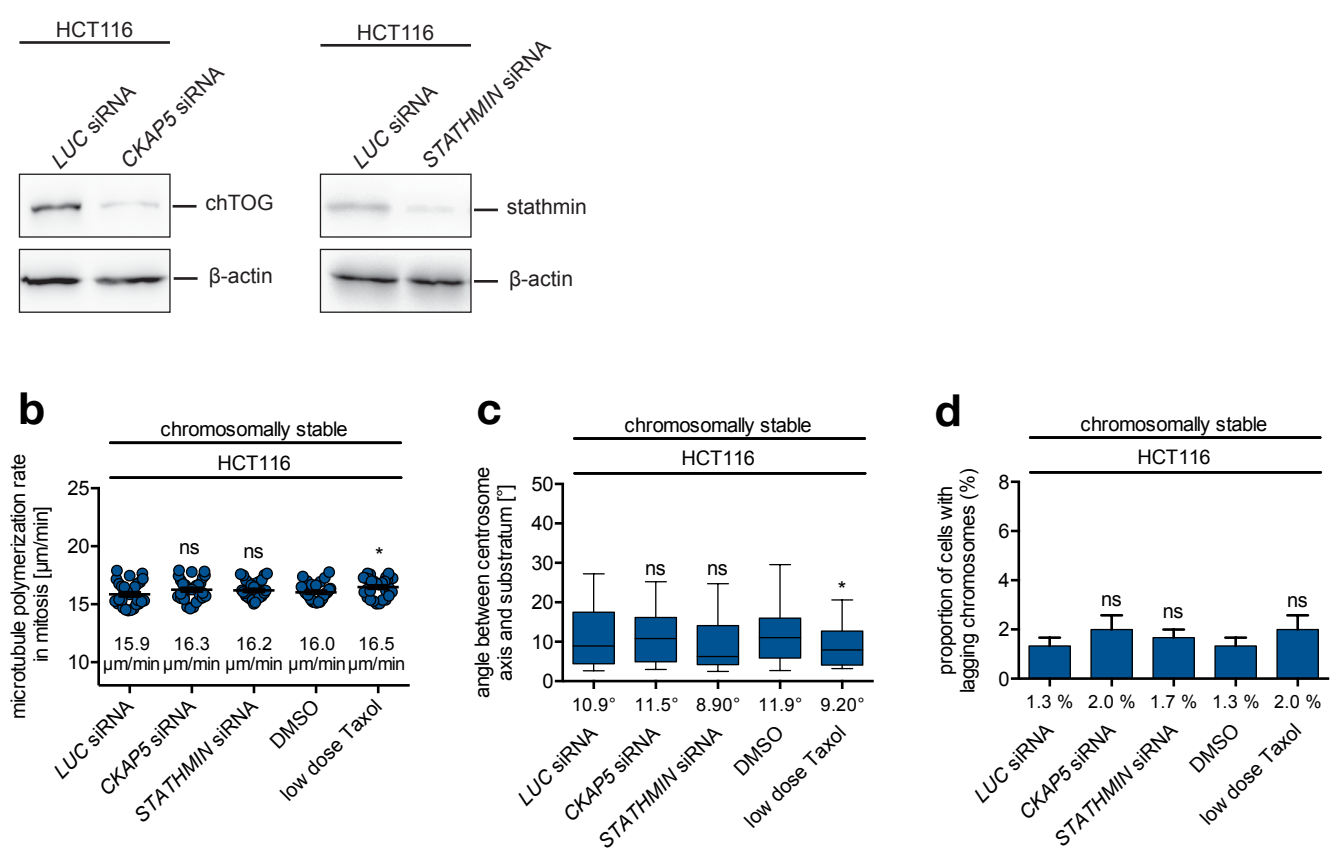
Fig. 3.4 Partial depletion of CKAP5, STATHMIN or low doses of Taxol have no effect on microtubule plus-end assembly rates, spindle axis alignment and lagging chromosomes in chromosomally stable HCT116 cells.

(a) Representative western blot showing partial depletion of and STATHMIN and CKAP5 in HCT116 cells after siRNA transfection. $\beta$-actin was used as a loading control. (b) Measurement of microtubule plus-end assembly rates in HCT116 cells during mitosis. $48 \mathrm{~h}$ prior measurement cells were transfected with either CKAP5, STATHMIN or LUCIFERASE (LUC) siRNA. For measurement of microtubule plus-end dynamics, $0.2 \mathrm{nM}$ Taxol was added along with $2 \mu \mathrm{M}$ DME for $1 \mathrm{~h}$. Scatter dot plots show mean values \pm SEM of 20 microtubules analyzed per cell of three independent experiments (30 cells analyzed in total, $t$-test). (c) Measurement of the angle between the centrosome axis and substratum in prometaphase. Cells were transfected as described in (b). $0.2 \mathrm{nM}$ Taxol was added $16 \mathrm{~h}$ prior fixation. Cells were seeded on fibronectin coated coverslips and were fixed and stained for immunofluorescence microscopy. The box and whisker diagram show the median (mean values were added), the 25th to 75th percentile and the minimum and maximum values ( $n=3,60$ cells analyzed in total, $t$-test). (d) Quantification of lagging chromosomes. Cells were transfected as described in (b). $8 \mathrm{~h}$ prior fixation $0.2 \mathrm{nM}$ Taxol was added. Cells were synchronized at G1/S-phase by a double thymidine block. After releasing the cells into the cell cycle for $8.5 \mathrm{~h}$, cells were fixed and stained for immunofluorescence microscopy. Bar graphs show the proportion of cells exhibiting lagging chromosomes ( $n=3$, total amount of 300 analyzed anaphases, mean values $\pm S E M, t$-test). Indicated $p$-values refer to corresponding control cells. Indicated $p$-values refer to corresponding control cells. ns $=$ not significant; $p>0.05 ;{ }^{*}: p \leq 0.05$;

Repression of CKAP5 and STAHMIN in HCT116 cells was confirmed by western blot analysis (Fig. 3.4a). First, it was analyzed whether a partial depletion of ch-TOG and stathmin or low doses of Taxol had an effect of microtubule plus-end assembly rates, spindle axis alignment and lagging chromosomes in chromosomally stable HCT116 cells (Fig. 3.4). These cells showed an average rate of microtubule plus-end assembly of $16.0 \mu \mathrm{m} / \mathrm{min}$ (Fig. 3.4b), which was not significantly altered after downregulation of CKAP5, STATHMIN or a treatment with low doses of Taxol (Fig. 3.4b). Likewise, there was no effect on spindle axis alignment in prometaphase (Fig. 3.4b) and the proportion of cells with lagging chromosomes (Fig. 3.4d).

Next, chromosomally unstable SW480 cells and SW620 cells were analyzed after repression of CKAP5 and STATHMIN or low doses of Taxol (Fig. 3.5). Partial depletion of ch-TOG and stathmin was verified by western blot analysis (Fig. 3.5a). While SW480 control cells showed an average rate of microtubule plus-end assembly of $21 \mu \mathrm{m} / \mathrm{min}$, repression of CKAP5 and STATHMIN or low doses of Taxol reduced microtubules plus-end assembly rates to an average of $16.2 \mu \mathrm{m} / \mathrm{min}$, which was comparable to microtubule plus-end assembly rates observed in chromosomally stable HCT116 cells (Fig. 3.5b). Likewise, partial depletion of ch-TOG and stathmin or Taxol addition, led to a significant decrease of microtubule plus-end assembly rates in SW620 (Fig. 3.5b). Intriguingly, restoration of proper microtubule plus-end assembly rates resulted in proper spindle axis alignment in prometaphase in SW480 cells and SW620 cells (Fig. 3.5c). Here, the average angle of spindle axis alignment in both cell lines of $21.0^{\circ}$ was almost reduced by half (Fig. 3.5b). Moreover, rescue of abnormal microtubule dynamics was also sufficient to decrease the number 
of cells exhibiting lagging chromosomes from $5.8 \%$ to $2.6 \%$ (Fig. 3.5c). Thus, these data confirm previous findings from our lab (Berger, 2016).

a
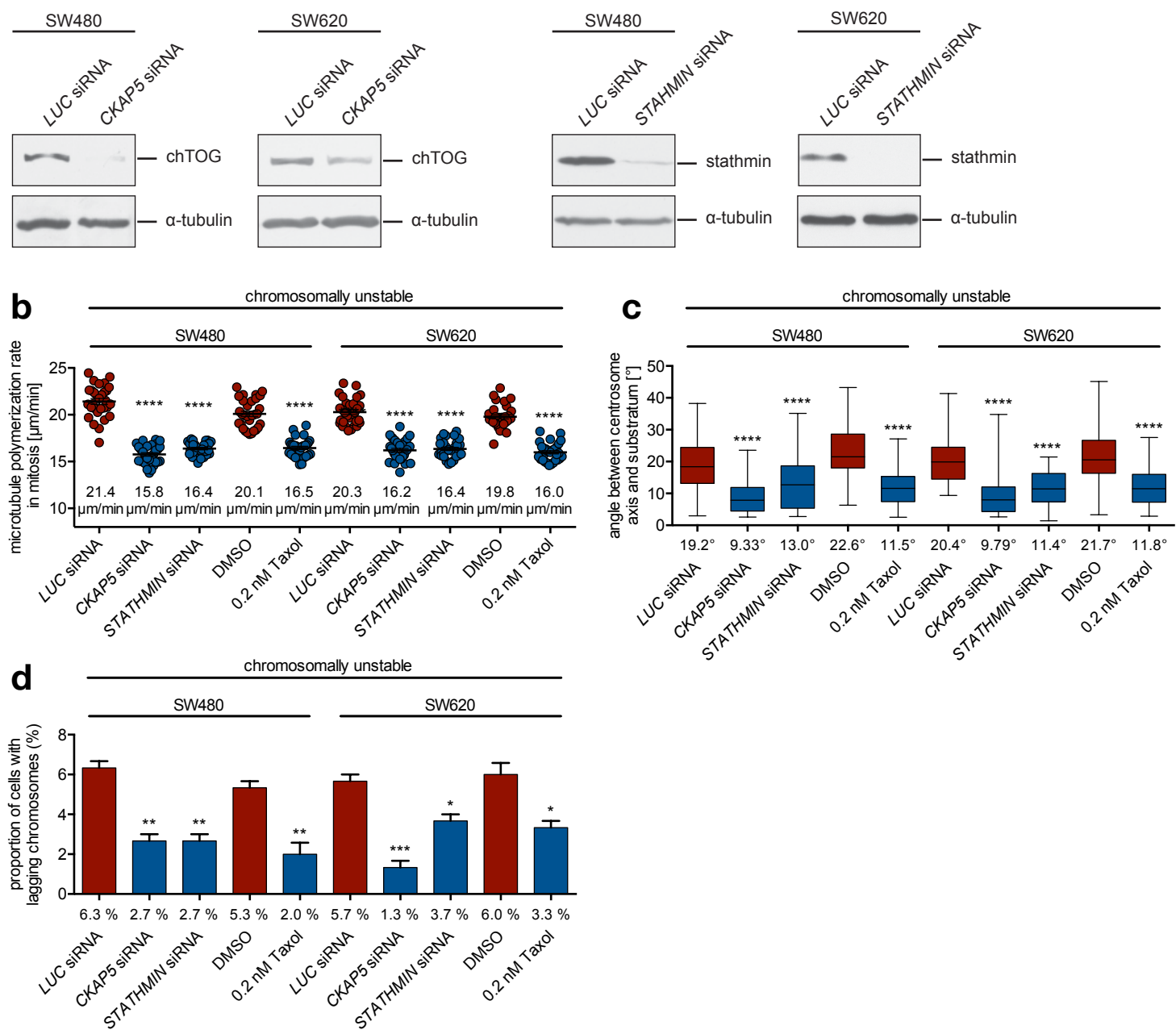

Fig. 3.5 Restoration of proper microtubule dynamics in CIN cells leads to proper spindle axis alignment in prometaphase and decreases the amount of lagging chromosomes in anaphase.

(a) Repression of STATHMIN and CKAP5 in SW480 cells and SW620 cells after siRNA transfection. atubulin was used as a loading control. (b) EB3 tracking in SW480 cells and SW620 cells during mitosis. Cell were transfected with CKAP5, STATHMIN or LUCIFERASE (LUC) siRNAs $48 \mathrm{~h}$ prior measurement. $0.2 \mathrm{nM}$ Taxol were simultaneously with $2 \mu \mathrm{M}$ DME $1.5 \mathrm{~h}$ before measurement. Scatter dot plots show mean values \pm SEM of 20 microtubules analyzed per cell of three independent experiments ( 30 cells analyzed in total, $t$ test). (c) Analysis of the spindle axis alignment in prometaphase. Cells were transfected as described in (b) and were grown on fibronectin coated coverslips. To restore proper microtubule plus-end assembly rates $0.2 \mathrm{nM}$ Taxol was added $16 \mathrm{~h}$ prior fixation. The following day cells were fixed and stained for $\alpha$-tubulin and $\mathrm{Y}$-tubulin for immunofluorescence microscopy. The box and whisker diagram show the median (mean values were added), the 25th to 75th percentile and the minimum and maximum values ( $n=3,60$ cells analyzed in total, $t$-test). (d) Quantification of lagging chromosomes. Cells were transfected as described in (b) and synchronized at G1/S-phase by a double thymidine block. $0.2 \mathrm{nM}$ Taxol was added at the time cells were released into the cell cycle for $8.5 \mathrm{~h}$. Afterwards, cells were fixed and stained for immunofluorescence microscopy with antibodies detecting $\alpha$-tubulin and CENP-C. Bar graphs show the proportion of cells exhibiting a colocalization of CENP-C and a lagging chromatid $(n=3$, total amount of 300 analyzed 
anaphases, mean values \pm SEM, $t$-test). Indicated $p$-values refer to corresponding control cells. ${ }^{* * * *}: p \leq$ $0.0001 ;{ }^{* *}: p \leq 0.01 ;{ }^{*}: p \leq 0.05$

To further strengthen the correlation between enhanced microtubule plus-end assembly rates and spindle axis misalignment in prometaphase, the effect of an overexpression of CKAP5, STATHMIN or of low doses of nocodazole on microtubule plus-end assembly rates, spindle axis alignment and lagging chromosomes was analyzed. Even though nocodazole is described as an microtubule depolymerizing and destabilizing agent, sub nanomolar concentrations were shown to induce enhanced microtubule plus-end assembly rates in HCT116 cells (Ertych et al., 2014). First, overexpression of CKAP5 and STAHMIN was verified by western blot analysis (Fig. 3.6a.) Microtubule plus-end assembly rates were increased from $16.4 \mu \mathrm{m} / \mathrm{min}$ to $19.3 \mu \mathrm{m} / \mathrm{min}$ after CKAP5 overexpression and to $20.4 \mu \mathrm{m} / \mathrm{min}$ after STATHMIN overexpression (Fig. 3.6b). Likewise, low doses of nocodazole enhanced microtubule plus-end assembly rates from $16.3 \mu \mathrm{m} / \mathrm{min}$ to $19.2 \mu \mathrm{m} / \mathrm{min}$ (Fig. 3.6b). Thus, these results verify previous data from Ertych et al. (Ertych et al., 2014). The induction of enhanced microtubule plus-end dynamics went along with an induction of spindle axis misalignment in HCT116 cells in prometaphase. Here, the average spindle angle was significantly increased from $11^{\circ}$ to $20^{\circ}$ (Fig. 3.6c). Accordingly, the proportion of cells with lagging chromosomes was increased after overexpression of CKAP5 and STATHMIN or after adding low doses of nocodazole. While control HCT116 cells exhibited $1.7 \%$ of cells with lagging chromosomes, CKAP5 or STATHMIN overexpression resulted in $4.7 \%$ and $4.3 \%$ lagging chromosomes, respectively (Fig. 3.6d). Also, low doses of nocodazole resulted in chromosome mis-segregation (Fig. 3.6d).

The above performed experiments demonstrate the causal role between microtubule plus-end assembly rates, spindle axis alignment in prometaphase and the amount of lagging chromosomes. On the one hand, restoring proper microtubule plus-end assembly rates in chromosomally unstable SW480 cells and SW620 cells restored proper spindle axis alignment in prometaphase and reduced the generation of lagging chromosomes in anaphase. On the other hand, the induction of enhanced microtubule plus-end assembly rates in chromosomally stable HCT116 cells was sufficient to induce both, spindle axis misalignment in prometaphase and lagging chromosomes in anaphase. 
a

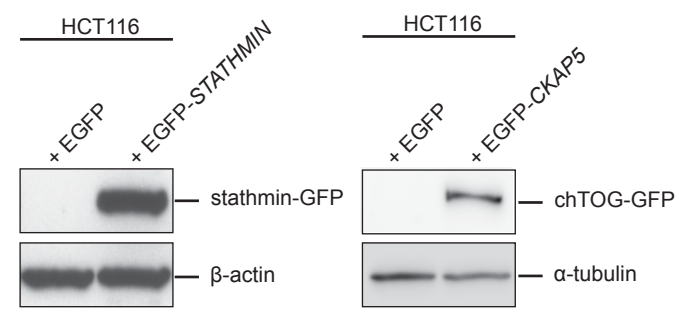

b

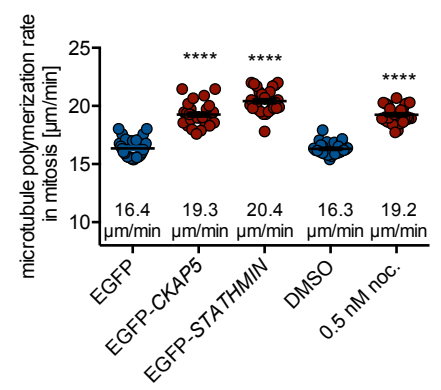

C

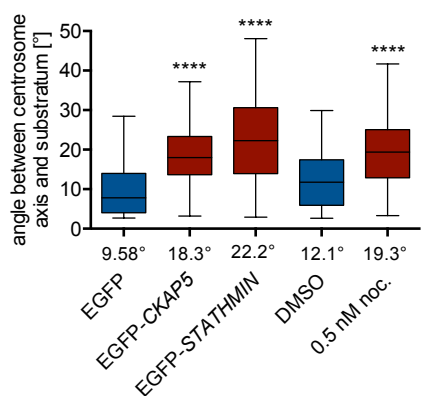

d

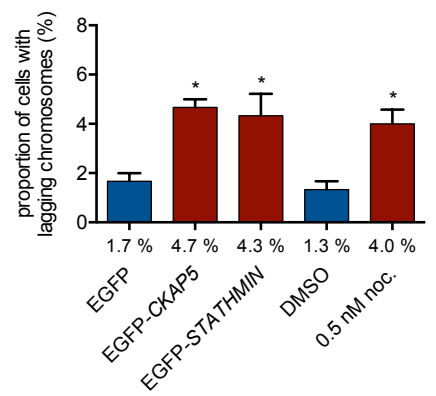

Fig. 3.6 Induction of enhanced microtubule plus-end assembly rates induces spindle axis misalignment and an increased proportion of cells with lagging chromosomes in chromosomally stable HCT116 cells.

(a) Representative western blot showing overexpression of CKAP5 and STATHMIN in HCT116 cells. atubulin or $\beta$-actin was used as a loading control. (b) Measurement of microtubule plus-end assembly rates of HCT116 cells in mitosis after overexpression of CKAP5, STATHMIN or low doses of nocodazole (noc). $48 \mathrm{~h}$ prior measurement cells were transfected with $2.5 \mu \mathrm{g}$ of CKAP5 by using Lipofectamine or with $10 \mu \mathrm{g}$ of STATHMIN by electroporation. For nocodazole treatment, $0.5 \mathrm{nM}$ nocodazole was added along with 2 $\mu M$ DME $1 \mathrm{~h}$ before EB3 tracking. Scatter dot plots show mean values \pm SEM of 20 microtubules analyzed per cell of three independent experiments (30 cells analyzed in total, $t$-test). (c) Measurement of the angle between the centrosome axis and substratum of HCT116 cells in prometaphase. Cells were transfected as described in (b) or were treated for $16 \mathrm{~h}$ with $0.5 \mathrm{nM}$ nocodazole. Cells were grown on fibronectin coated coverslips, fixed and stained for $\alpha$-tubulin and $y$-tubulin for immunofluorescence microscopy. The box and whisker diagram show the median (mean values were added), the 25th to 75th percentile and the minimum and maximum values ( $n=3,60$ cells analyzed in total, $t$-test). (d) Quantification of lagging chromosomes. Transfection was performed as described in (b) or $0.5 \mathrm{nM}$ nocodazole was added for $8 \mathrm{~h}$. Cells were synchronized at G1/S-phase by a double thymidine block and fixed after a release of $8.5 \mathrm{~h}$. For immunofluorescence microscopy, cells were stained for a-tubulin and CENP-C. Bar graphs show the proportion of cells with lagging chromosomes $(n=3$, total amount of 300 analyzed anaphases, mean values \pm SEM, $t$-test). Indicated $p$-values refer to corresponding control cells. ${ }^{* * * *}: p \leq 0.0001 ;{ }^{*}: p \leq 0.05$

\subsection{Spindle axis misalignment promotes chromosome mis-segregation in chromosomally stable HCT116 cells}

Mitotic spindle orientation and positioning is still a subject of current research and several mechanisms are proposed to influence spindle orientation (di Pietro et al., 2016). The classical pathway involves a complex consisting of the heterotrimeric protein Gai, NuMA1 and LGN, which serves as an anchor for the minus-end directed motor dynein that pulls on astral microtubules to 
orient the mitotic spindle (di Pietro et al., 2016). In order to analyze whether spindle axis misalignment per se leads to lagging chromosomes in anaphase, LGN was partially depleted in chromosomally stable HCT116 cells, which was verified by western blot analyses (Fig. 3.7a). As intended, the angle of spindle axis alignment was increased from $10.9^{\circ}$ to $22.4^{\circ}$ in prometaphase and from $5.05^{\circ}$ to $8.72^{\circ}$ in metaphase (Fig. 3.7c). Moreover, induction of spindle misalignment in HCT116 cells resulted in an increased amount of cells with lagging chromosomes in anaphase (Fig. 3.7d). However, repression of $L G N$ caused an increase of microtubule plus-end rates from $16.4 \mu \mathrm{m} / \mathrm{min}$ to $19.7 \mu \mathrm{m} / \mathrm{min}$ (Fig. 3.7b). Thus, this experiment did not verify a function of spindle axis misalignment per se for the induction of lagging chromosomes since partial depletion of LGN also caused enhanced microtubule plus-end assembly rates.

That's why another approach had to be established to interfere with the $\mathrm{Ga}_{\mathrm{i}}$, LGN and NuMA1 complex. It was shown that a GEF called Ric-8A modulates the activity and localization of $\mathrm{Ga}_{\mathrm{i}}$ whereby it assures proper spindle orientation (Couwenbergs et al., 2004; David et al., 2005). Moreover, it was found that pertussis toxin from Bordetella pertussis inhibits the binding of Ric-8A to $\mathrm{Ga}_{\mathrm{i}}$ and thereby disrupts normal spindle alignment in metaphase cells (Woodard et al., 2010).

a

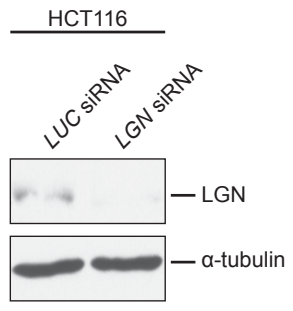

b

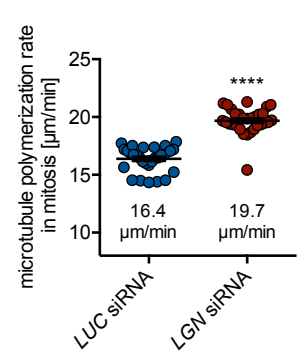

C

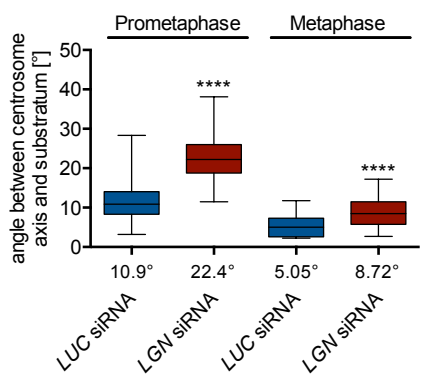

d

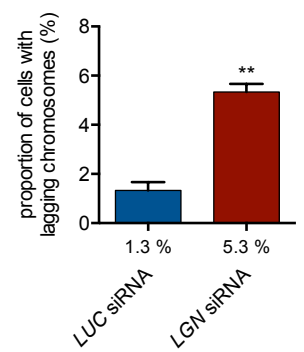

Fig. 3.7 Induction of spindle axis misalignment by LGN knockdown induces lagging chromosomes. (a) Depletion of LGN in HCT116 cells $48 \mathrm{~h}$ after siRNA transfection was confirmed by western blot analysis. a-tubulin was used as a loading control. (b) Measurements of microtubule plus-end assembly rates in mitosis. Cells were transfected with LGN siRNA or LUCIFERASE (LUC) siRNA as a control. For live cell analysis cells were treated with $2 \mu \mathrm{M}$ DME for $1 \mathrm{~h}$. Scatter dot plots show mean values \pm SEM of 20 microtubules analyzed per cell of three independent experiments (30 cells analyzed in total, $t$-test). (c) Measurement of the spindle axis alignment in prometaphase and metaphase. Cells were transfected as described in (b), seeded on fibronectin coated coverslips and stained for immunofluorescence microscopy. The box and whisker diagram show the median (mean values were added), the $25^{\text {th }}$ to $75^{\text {th }}$ percentile and the minimum and maximum values ( $n=3,60$ cells analyzed in total, $t$-test). (d) Occurrence of lagging chromosomes. siRNA transfection was performed as described in (b). To accumulate cells in anaphase, cells were synchronized at $\mathrm{G}_{1} / \mathrm{S}$-phase by a double thymidine block. Cells were fixed and stained for immunofluorescence microscopy after $8.5 \mathrm{~h}$ of cell cycle release. Bar graphs show the amount of cells CENP-C positive chromatids lagging behind in anaphase $(n=3$, total amount of 300 analyzed anaphases, mean values \pm SEM, t-test). Indicated $p$-values refer to corresponding control cells. ${ }^{* * *}: p \leq 0.0001 ;{ }^{* *} p \leq$ 0.01 
Therefore, the effect of pertussis toxin on spindle axis alignment was analyzed. Pertussis toxin was sufficient to increase the angle of spindle axis alignment in a concentration dependent manner. Already $100 \mathrm{ng} / \mathrm{ml}$ pertussis toxin increased the angle of spindle axis alignment from 12.1 ${ }^{\circ}$ to $19.5^{\circ}$, while $200 \mathrm{ng} / \mathrm{ml}$ and $400 \mathrm{ng} / \mathrm{ml}$ pertussis toxin led to an angle of $24.8^{\circ}$ and $27.5^{\circ}$, respectively (Fig. 3.8b). This went along with an increased number of cells displaying lagging chromosomes. Here, $2.3 \%$ of control cells exhibited lagging chromosomes while addition of pertussis toxin increased the proportion of cells with lagging chromosomes from $4.7 \%$ up to $6.3 \%$ (Fig. 3.8c). Importantly, pertussis toxin had no effect on microtubule plus-end assembly rates in HCT116 cells (Fig. 3.8a).

These data indicate, that spindle axis misalignment in prometaphase per se promotes the generation of lagging chromosomes independent of microtubule plus-end assembly rates.
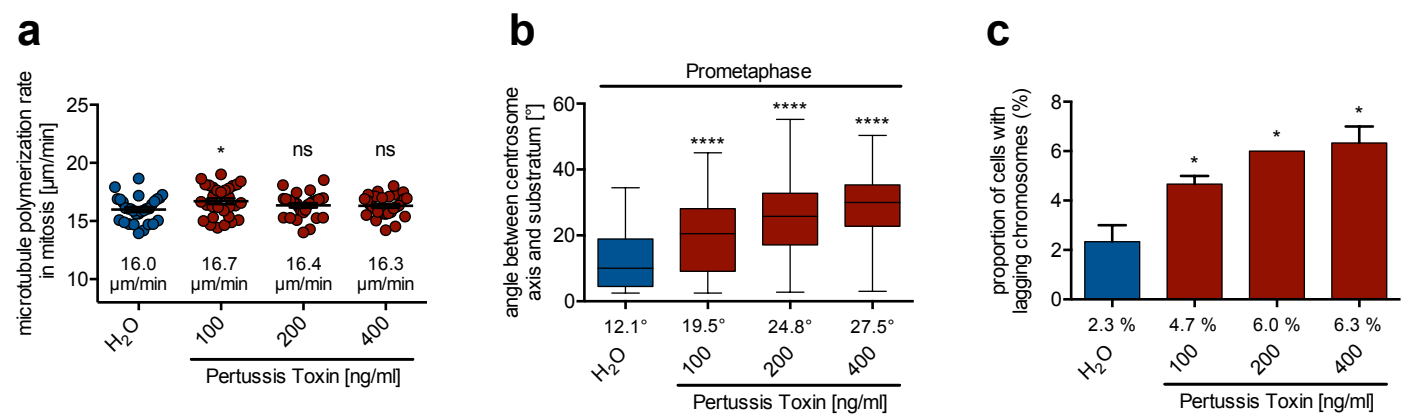

Fig. 3.8 Interfering with the spindle tethering machinery induces spindle axis misalignment and lagging chromosomes.

(a) Measurement of microtubule plus-end assembly rates in mitosis. For EB3 tracking cells were incubated for $1 \mathrm{~h}$ with $2 \mu \mathrm{M}$ DME and $100-400 \mathrm{ng} / \mathrm{ml}$ pertussis toxin. Scatter dot plots show mean values \pm SEM of 20 microtubules analyzed per cell of three independent experiments (30 cells analyzed in total, $t$-test). (b) Measurement of the angle between the centrosome axis and substratum in prometaphase. Cells were seeded on fibronectin coated coverslips and treated with $100-400 \mathrm{ng} / \mathrm{ml}$ pertussis toxin for $1 \mathrm{~h}$. Cells were fixed and stained for immunofluorescence microscopy. The box and whisker diagram show the median (mean values were added), the $25^{\text {th }}$ to $75^{\text {th }}$ percentile and the minimum and maximum values $(n=3,60$ cells analyzed in total, $t$-test). (c) Analysis of cells with lagging chromosomes. Cells were synchronized at $\mathrm{G}_{1} / \mathrm{S}$ phase by a double thymidine block. After releasing the cells into the cell cycle $100-400 \mathrm{ng} / \mathrm{ml}$ pertussis toxin was added $1 \mathrm{~h}$. After for $8.5 \mathrm{~h}$ of release, cells were fixed and stained for immunofluorescence microscopy. Bar graphs show the proportion of cells with lagging chromosomes $(n=3$, total amount of 300 analyzed anaphases, mean values \pm SEM, $t$-test). $n s=$ not significant, $p>0.05 ;{ }^{*}: p \leq 0.05 ;{ }^{* * * *}: \leq 0.0001$

To further investigate if spindle axis misalignment is sufficient to induce CIN in chromosomally stable HCT116 cells, single cell clones were generated. For this purpose, HCT116 cells were cultivated for 30 generations in the presence of $100 \mathrm{ng} / \mathrm{ml}$ pertussis toxin or $\mathrm{H}_{2} \mathrm{O}$ as a control. Control clones showed an average angle of spindle axis alignment of $10.5^{\circ}$ while pertussis toxin treated single cell clones exhibited an average angle of $18.3^{\circ}$ (Fig. 3.9a). Accordingly, long term 
treatment of pertussis toxin resulted in an increased proportion of cells with lagging chromosomes (Fig. 3.9b).

These results suggest, that spindle axis misalignment induced by long term treatment with pertussis toxin facilitates whole chromosome mis-segregation in chromosomally stable HCT116 cells. Moreover, these data suggest, that with increasing spindle axis misalignment, the proportion of cells with lagging chromosomes also increases, which emphasizes the importance of proper spindle orientation for chromosome segregation.

a

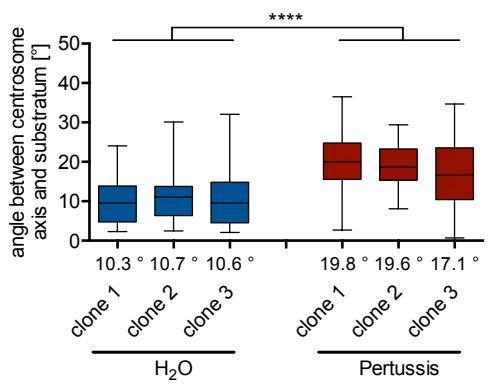

b

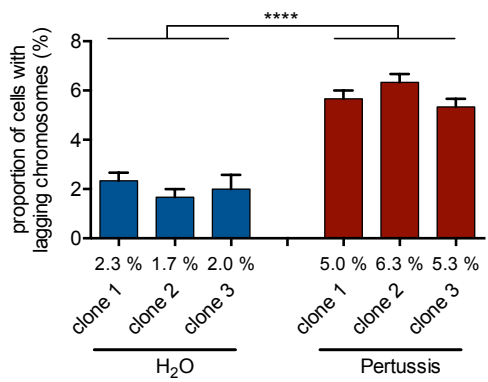

Fig. 3.9 Pertussis toxin-induced spindle axis misalignment in prometaphase causes whole chromosome mis-segregation in HCT116 cells.

(a) Spindle axis alignment in prometaphase of HCT116 single cell clones generated for 30 days in the presence of $100 \mathrm{ng} / \mathrm{ml}$ pertussis toxin or $\mathrm{H}_{2} \mathrm{O}$ as a control. Cells were grown on fibronectin coated coverslips, fixed and stained for immunofluorescence microscopy. The box and whisker diagram show the median (mean values were added), the $25^{\text {th }}$ to $75^{\text {th }}$ percentile and the minimum and maximum values ( $n=3,60$ cells analyzed in total, $t$-test). (b) Occurrence of lagging chromosomes in HCT116 single cell clones generated as described in (a). For an accumulation of anaphases, cells were synchronized at $\mathrm{G}_{1} / \mathrm{S}$-phase by a double thymidine block and released into the cell cycle for $8.5 \mathrm{~h}$. Afterwards, cells were fixed and stained for immunofluorescence microscopy. Bar graphs display the amount of lagging chromosomes $(n=3$, with a total of 300 anaphase cells, mean values \pm SEM, $t$-test). ${ }^{* * * *}: \leq 0.0001$. Clones were generated and kindly provided by L. Hennecke, Bastians lab, Institute for Molecular Oncology, Section of Cellular Oncology.

\subsection{Deregulation of the actin cortex cytoskeleton leads to spindle axis misalignment in prometaphase and promotes the generation of lagging chromosomes.}

Besides the $\mathrm{Ga}_{\mathrm{i}}, \mathrm{LGN}$ and NuMA pathway, the actin cortex also plays an important role for spindle orientation (Kunda \& Baum, 2009). That's why it was of interest to investigate if the mitotic actin cytoskeleton is involved in spindle axis alignment. Therefore, chromosomally stable HCT116 cells and chromosomally unstable SW480 cells as well as SW620 cells were analyzed after an incubation with Latrunculin A, which is a well-established inhibitor of actin polymerization (Morton, Ayscough, \& Mclaughlin, 2000). Intriguingly, SW480 cells and SW620 cells, which are characterized by spindle axis misalignment, Latrunculin A treatment restored proper spindle axis alignment during prometaphase. In these cells, already $0.75 \mu \mathrm{M}$ Latrunculin A led to a significantly 
rescue of the average spindle angle from $21^{\circ}$ to $14^{\circ}$ in both chromosomally unstable cell lines (Fig. 3.10a). Moreover, in SW480 cells and SW620 cells the proportion of cells with lagging chromosomes was reduced upon Latrunculin A treatment (Fig. 3.10c). Interestingly, the same treatment led to an induction of spindle axis misalignment and promoted the generation of lagging chromosomes in chromosomally stable HCT116 cells (Fig. 3.10a). To further analyze the role of the actin cytoskeleton for spindle axis alignment, Jasplakinolide was used as a promotor for actin polymerization (Bubb et al., 1994). Intriguingly, enhanced actin polymerization induced spindle axis misalignment in chromosomally stable HCT116 cells and RKO cells, whereby the spindle angle was doubled in comparison to control cells (Fig. 3.10b).
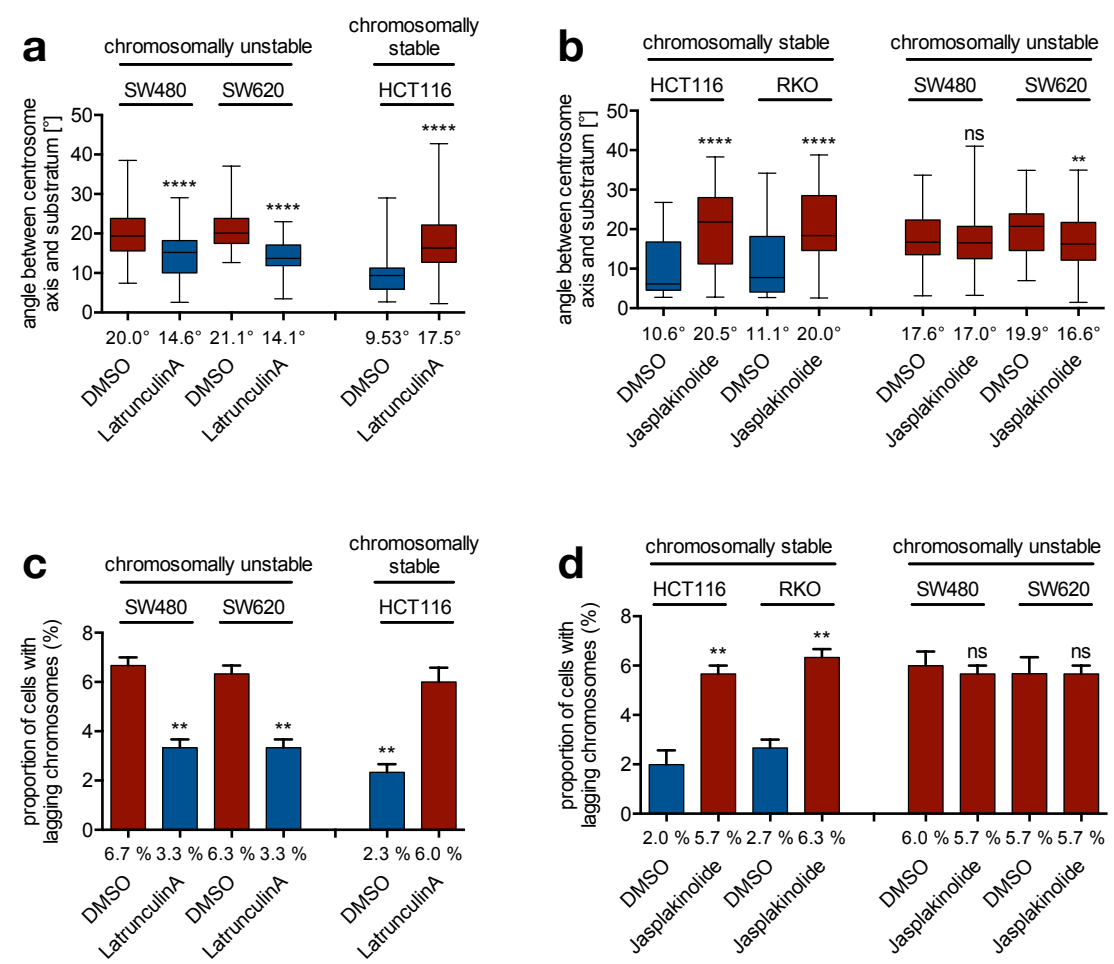

Fig. 3.10 Modulation of actin polymerization affects spindle axis alignment in prometaphase and chromosome segregation.

(a-b) Spindle axis alignment in chromosomally stable HCT116 cells, RKO cells, and chromosomally unstable SW480 cells and SW620 cells during prometaphase. For treatments and analysis, cells were grown on fibronectin coverslips in the presence of $0.75 \mu \mathrm{M}$ Latrunculin A for $4 \mathrm{~h}$ (a) or $100 \mathrm{nM}$ Jasplakinolide for $16 \mathrm{~h}$ (b) and were fixed and stained for immunofluorescence microscopy. The box and whisker diagram show the median (mean values values were added), the 25th to 75th percentile and the minimum and maximum values $(n=3,60$ cells analyzed in total, $t$-test). (c-d) Proportion of cells showing lagging chromosomes after treatment with $0.75 \mu \mathrm{M}$ Latrunculin A for $4 \mathrm{~h}$ (c) or $100 \mathrm{nM}$ Jasplakinolide for $8 \mathrm{~h}$ (d). Cells were synchronized at G1/S-phase by a double thymidine block and released into the cells into the cell cycle. After $8.5 \mathrm{~h}$ cells were fixed and stained for immunofluorescence microscopy. Bar graphs show the proportion of cells exhibiting lagging chromosomes in anaphase $(n=3$, total amount of 300 analyzed anaphases, mean values \pm SEM, $t$-test). $n s=$ not significant, $p>0.05 ;{ }^{* *}: p \leq 0.01 ;{ }^{* * *}: \leq 0.0001$ 
Interestingly, Jasplakinolide had no effect on spindle axis alignment in SW480 cells and in SW620 cells (Fig. 3.10b). Moreover, the amount of cells exhibiting lagging chromosomes was not changed in SW480 cells and SW620 cells whereas the addition of Jasplakinolide increased chromosome mis-segregation in chromosomally stable cell lines (Fig. 3.10d).

These results verify a role of the mitotic actin cytoskeleton for spindle axis alignment in prometaphase. Given the fact that the addition of Jasplakinolide had no impact on spindle axis alignment in SW480 cells and SW620 cells, these results indicate that chromosomally unstable cells already exhibit enhanced actin polymerization. This is supported by the fact that spindle axis misalignment was induced in HCT116 cells upon addition of Jasplakinolide, while inhibition of actin polymerization restored proper spindle axis alignment in SW480 cells and SW620 cells.
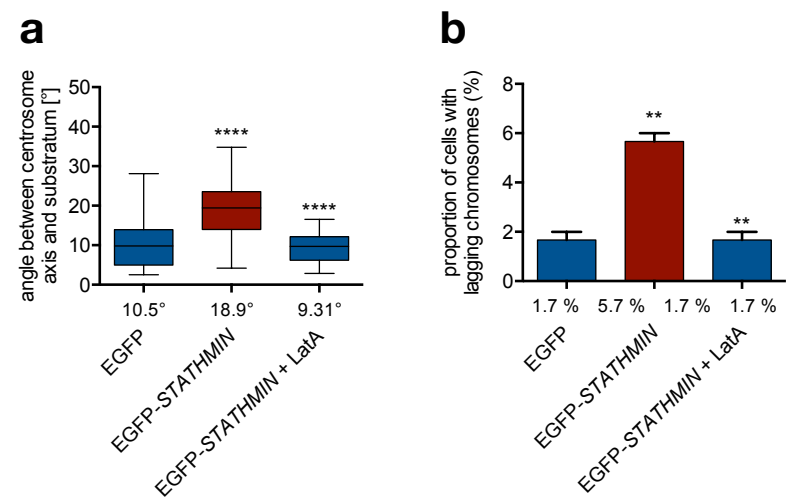

Fig. 3.11 Actin polymerization is mediated by increased microtubule plus-end assembly rates to regulate spindle axis alignment and chromosome segregation.

(a) Measurement of the angle between the centrosome axis and substratum in HCT116 cells during prometaphase after overexpression of STATHMIN and an additional incubation with Latrunculin A. Cells were transfected with $10 \mu \mathrm{g}$ STATHMIN $48 \mathrm{~h}$ prior fixation. For actin depolymerization cells were grown in the presence of $0.75 \mu \mathrm{M}$ Latrunculin A for $4 \mathrm{~h}$ prior fixation. Afterwards, cells were fixed and stained for immunofluorescence microscopy. The box and whisker diagram show the median (mean values values were added), the 25th to 75th percentile and the minimum and maximum values ( $n=3,60$ cells analyzed in total, $t$-test). (b) For the quantification of lagging chromosomes cells were transfected as described in (a). To accumulate cells in anaphase, cells were synchronized at G1/S-phase by a double thymidine block and $0.75 \mu \mathrm{M}$ Latrunculin A was added for $4 \mathrm{~h}$ after releasing the cells into the cell cycle. Following $8.5 \mathrm{~h}$, cells were fixed and stained for immunofluorescence microscopy. Bar graphs show the proportion of cells exhibiting a CENP-C positive chromatid lagging behind in anaphase $(n=3$, total amount of 300 analyzed anaphases, mean values \pm SEM, $t$-test). ${ }^{* *}: p \leq 0.01 ;^{* * * *}: \leq 0.0001$

To investigate whether CIN cells exhibit increased actin polymerization mediated spindle axis misalignment due to enhanced microtubule plus-end assembly rates, spindle axis alignment and lagging chromosomes were analyzed after inducing enhanced microtubule plus-end assembly rates in chromosomally stable HCT116 cells and an additional treatment with Latrunculin A. As expected, overexpression of STATHMIN resulted in spindle axis misalignment by increasing the angle from $10.5^{\circ}$ to $18.9^{\circ}$ (Fig. 3.11a). Importantly, this misalignment of the spindle axis was 
corrected by the addition of Latrunculin A (Fig. 3.11a). Likewise, the amount of cells with lagging chromosomes in HCT116 cells was almost doubled after STATHMIN overexpression while inhibition of actin polymerization reduced stathmin induced chromosome mis-segregation (Fig. 3.11b).

Thus, it is likely that increased microtubule plus-end assembly rates lead to enhanced actin polymerization, which promotes spindle axis misalignment and thus, the generation of lagging chromosomes.

\subsection{Decreased cortical tension during mitosis is responsible for spindle axis misalignment in prometaphase.}

Recently, a role of the actin cytoskeleton for cortical tension was reported. Chugh et al. showed that actin cortex thickness and the composition of the actin cortex along with myosin II activity are crucial for proper cortical tension during mitosis (Chugh et al., 2017). In general, mitotic cells exhibit a higher cortical tension than cells in interphase, which is achieved by an interplay between the actin cytoskeleton, myosin activity and osmotic pressure (Stewart et al., 2011). Since the actin cytoskeleton turned out to be crucial for spindle axis alignment in prometaphase, the question arose if cortical tension is also relevant for spindle axis alignment. Thus, blebbistatin, a wellestablished myosin II inhibitor (Kovács et al., 2004), which is able to decrease cell contractility (Fischer-Friedrich et al., 2014) was used to analyze the impact of cortical tension on spindle axis alignment and lagging chromosomes.

Inhibition of myosin II had neither an effect on microtubule plus-end assembly rates in HCT116 cells nor in SW480 cells and SW620 cells (Fig. 3.12a). However, blebbistatin had a strong impact on spindle axis alignment in prometaphase in HCT116 cells as wells as SW480 cells and SW620 cells. In chromosomal stable HCT116 cells, blebbistatin led to a concentration dependent induction of spindle misalignment by increasing the angle from $9.99^{\circ}$ over $13.5^{\circ}$ up to $16.4^{\circ}$ (Fig. 3.12b). Interestingly, the same treatment restored proper spindle axis alignment in chromosomally unstable SW480 cells and SW620 cells. Here, DMSO treated cells showed an angle of spindle axis alignment of $19.6^{\circ}$ and $21.2^{\circ}$, which decreased to $11.3^{\circ}$ and $11.6^{\circ}$ in SW480 cells and SW620 cells, respectively (Fig. 3.12b). The same effect was observed regarding the proportion of cells with lagging chromosomes whereby chromosome mis-segregation was induced upon blebbistatin treatment in HCT116 cells while myosin II inhibition reduced the amount of cells exhibiting lagging chromosomes in SW480 cells and SW620 cells (Fig. 3.12c). 

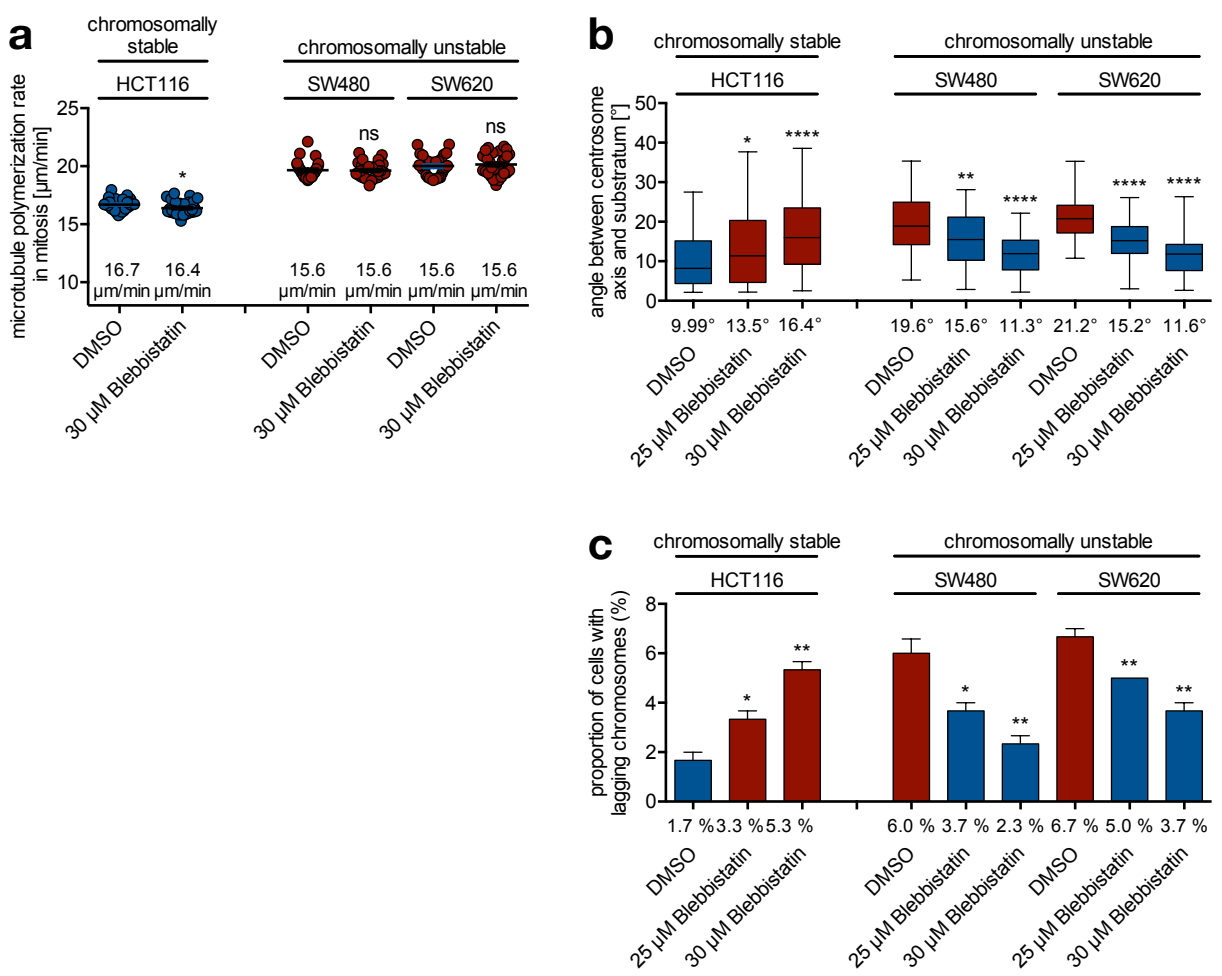

Fig. 3.12 Inhibition of myosin II induces spindle axis misalignment and lagging chromosomes in HCT116 cells and restores proper spindle axis alignment and chromosome segregation in CIN cells. (a) Microtubule plus-end assembly rates in HCT116 cells, SW480 cells and SW620 cells during mitosis. For EB3 tracking, cells were incubated for $1 \mathrm{~h}$ with $2 \mu \mathrm{M}$ DME and with $30 \mu \mathrm{M}$ blebbistatin. Scatter dot plots show mean values \pm SEM of 20 microtubules analyzed per cell of three independent experiments (30 cells analyzed in total, $t$-test). (b) Spindle axis alignment in HCT116 cells, SW480 cells and SW620 cells in prometaphase after inhibition of myosin II by $25 \mu \mathrm{M}$ and $30 \mu \mathrm{M}$ blebbistatin for $1 \mathrm{~h}$. For immunofluorescence microscopy, cells were grown on fibronectin coated coverslips and were fixed and stained with a-tubulin and $y$-tubulin. The box and whisker diagram show the median (mean values values were added), the 25th to 75th percentile and the minimum and maximum values ( $n=3,60$ cells analyzed in total, $t$-test). (c) Analysis of lagging chromosomes after myosin II inhibition. Therefore, cells were synchronized at G1/S-phase by a double thymidine block. Cells were released into the cell cycle for $8.5 \mathrm{~h}$ and $25 \mu \mathrm{M}$ and $30 \mu \mathrm{M}$ blebbistatin was added $1 \mathrm{~h}$ hour prior fixation. Afterwards, cells were fixed and stained for immunofluorescence microscopy. Bar graphs show the proportion of cells showing a colocalization of a CENP-C positive chromatid lagging behind ( $n=3$, total amount of 300 analyzed anaphases, mean values $\pm S E M, t$-test). Indicated $p$-values refer to corresponding control cells. $n s=$ not significant, $p>0.05 ;{ }^{*}: p \leq 0.05 ;{ }^{* *}: p \leq$ $0.01 ;{ }^{* * * *}: \leq 0.0001$

Nonmuscle myosin-II activity is regulated by phosphorylation of its regulatory light chain (RLC). Among others, rho associated kinase (ROCK) phosphorylates the RLC thereby activating myosin II. Moreover, ROCK phosphorylates myosin II light chain phosphatase, which also leads to activation of myosin II (Bresnick, 1999). To further strengthen the role of myosin mediated cortical tension, ROCK inhibition and its effect on spindle axis alignment and chromosome segregation was analyzed. Like blebbistatin, ROCK inhibition led to opposite effects on MIN/MSI and CIN cells. With increasing concentrations, the angle of spindle axis alignment in HCT116 cells increased from $9.33^{\circ}$ to $14.2^{\circ}$ whereas proper spindle axis alignment was restored in SW480 cells and 
SW620 cells upon ROCK inhibition (Fig. 3.13a). Here, DMSO treated cells showed an angle of spindle axis alignment of $23.0^{\circ}$ and $20.0^{\circ}$, which was decreased to $15.1^{\circ}$ and $10.9^{\circ}$ in SW480 cells and SW620 cells, respectively (Fig. 3.13a). In the same manner, ROCK inhibition promoted the generation of lagging chromosomes in HCT116 cells whereas chromosome mis-segregation was reduced in SW480 cells and SW620 cells (Fig. 3.13b).
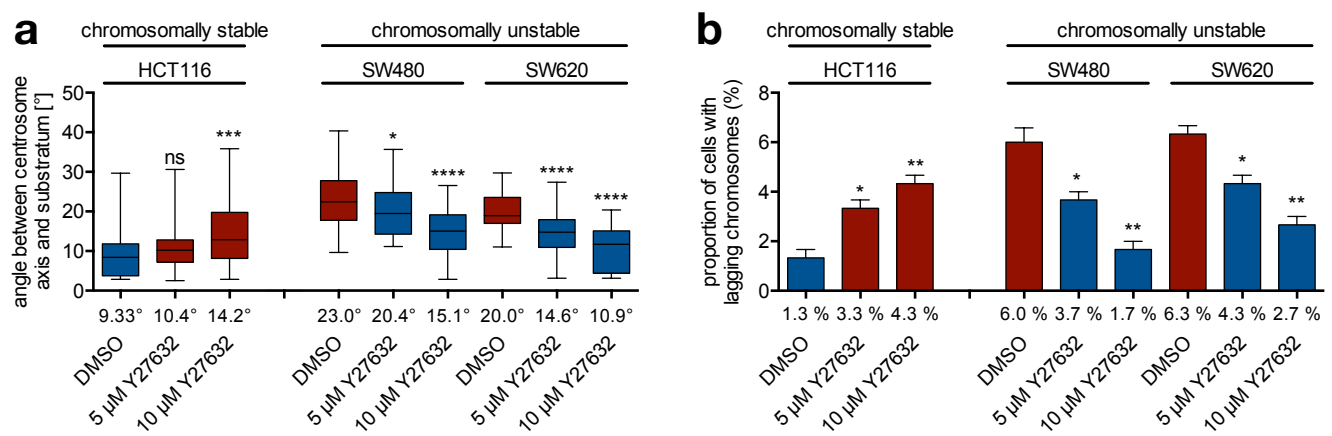

Fig. 3.13 Inhibition of ROCK restores proper spindle axis alignment and chromosome segregation in CIN cells and induces spindle axis misalignment and lagging chromosomes in HCT116 cells.

(a) HCT116 cells, SW480 cells and SW620 cells were analyzed regarding spindle axis alignment in prometaphase. Cells were grown on fibronectin coated coverslips and treated with $5 \mu \mathrm{M}$ and $10 \mu \mathrm{M} \mathrm{ROCK}$ inhibitor (Y27632) for $2 \mathrm{~h}$. Subsequently, cells were fixed and stained for immunofluorescence microscopy. The box and whisker diagram show the median (mean values were added), the 25th to 75th percentile and the minimum and maximum values ( $n=3,60$ cells analyzed in total, $t$-test). (b) Detection of lagging chromosomes after myosin II inhibition by $5 \mu \mathrm{M}$ or $10 \mu \mathrm{M}$ Y27632. Cells were synchronized at G1/S-phase by a double thymidine block. After releasing the cells into the cell cycle for $8.5 \mathrm{~h}$, cells were fixed and stained for immunofluorescence microscopy. ROCK inhibitor was added $2 \mathrm{~h}$ prior fixation. Bar graphs show the proportion of cells with lagging chromosomes $(n=3$, total amount of 300 analyzed anaphases, mean values \pm SEM, $t$-test). Indicated $p$-values refer to corresponding control cells. ns $=$ not significant, $p>0.05 ;{ }^{*}: p \leq$ $0.05 ;^{* *}: p \leq 0.01 ;^{* * *}: p \leq 0.001 ;^{* * * *}: \leq 0.0001$

These results indicate that MIN/MSI and CIN cells exhibit a difference in cortical tension during mitosis since myosin II inhibition had opposite effects on MIN/MSI and CIN cells.

To investigate this hypothesis, atomic force microscopy was used to measure cortical tension of mitotic HCT116 cells and SW620 cells. Indeed, chromosomally unstable SW620 cells exhibited reduced cortical tension during mitosis in comparison to HCT116 cells (Fig. 3.14). Blebbistatin was used as a control to lower cortical tension in HCT116 cells and resulted in a decrease of cortex tension, which was observed in SW620 cells (Fig. 3.14). Remarkably, restoring proper microtubule plus-end assembly rates by using low doses of Taxol, increased cortical tension in SW620 cells (Fig. 3.14).

These results suggest that reduced cortical tension in CIN cells facilitates spindle axis misalignment and the generation of lagging chromosomes since myosin II inhibition decreased 
cortex tension in HCT116 cells, which is accompanied with the induction of spindle axis misalignment and lagging chromosomes.

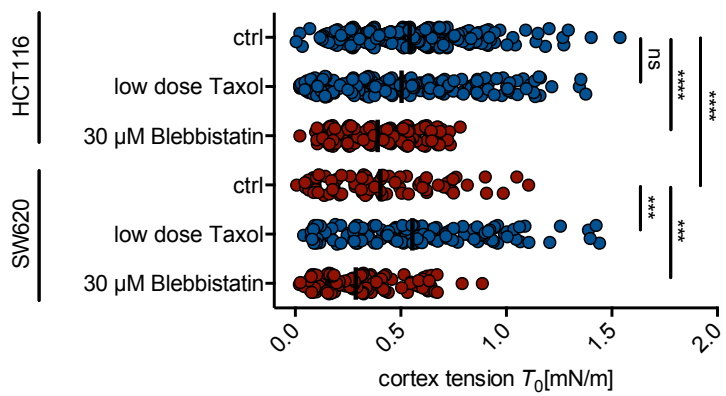

Fig. 3.14 Restoring proper microtubule plus-end assembly rates increases cortical tension $T_{0}$ of mitotic SW620 cells.

Measurement of cortical tension in mitotic HCT116 cells and SW620 cells by using atomic force microscopy. Cells were transfected $48 \mathrm{~h}$ prior measurement with $10 \mu \mathrm{g}$ GFP-H2B to facilitate the identification of mitotic cells. For restoring proper microtubule plus-end assembly rates $0.2 \mathrm{nM}$ Taxol was added $16 \mathrm{~h}$ prior measurement while myosin II was inhibited by $30 \mu \mathrm{M}$ blebbistatin $1 \mathrm{~h}$ prior measurement. Scatter dot plots show mean values as solid lines $(n \geq 20$ cells in at least three independent experiments, mean values \pm SEM, $t$-test). Results were generated and kindly provided by Dr. Bastian Rouven Brückner and Prof. Dr. Andreas Janshoff, Institute for Biophysical Chemistry in Göttingen. ns $=$ not significant, $p>0.05 ;{ }^{* * *}: p \leq$ $0.001 ;{ }^{* * * *}: \leq 0.0001$

\subsection{The Rac1-Arp2/3 pathway plays a role for spindle axis alignment exclusively in chromosomally unstable cells}

Since CIN cells revealed reduced cortical tension in comparison to MIN/MSI cells, it was of interest to analyze which factors might influence cortex tension in mitotic chromosomally stable and unstable cells. Interestingly, Ramanathan et al. showed that a RhoA-formin pathway, rather than Rac1-Arp2/3 signaling, is responsible for actin polymerization, which promotes the generation of cortical tension (Ramanathan et al., 2015). Moreover, Chugh and colleagues suggested that especially regulators for actin filament length, such as the formin DIAPH1 (diaphanous homolog 1) regulates actin cortex thickness to ensure proper cortex tension (Chugh et al., 2017). Since a hyperactive Rac1-Arp2/3 pathway was revealed as a trigger for CIN (Berger, 2016), the question arose whether this pathway causes reduced cortex tension in CIN cells due to Arp2/3 mediated actin polymerization, which results in spindle axis misalignment. While DIAPH1 mediated actin polymerization leads to unbranched actin filaments, actin nucleation by the Arp $2 / 3$ complex results in branched actin filaments. Thus, actin nucleators play a major role in defining the actin network structure (Blanchoin et al., 2014), which was shown to influence cortical tension besides myosin II activity (Chugh et al., 2017). Therefore, it was of great interest to analyze the role of branched 
actin polymerization mediated by Rhoa and DIAPH1 and unbranched actin polymerization based on the Arp2/3 complex, in the context of spindle axis alignment in prometaphase.

To address this question, HCT116 cells, SW480 cells and SW620 cells were treated with inhibitors for RhoA, Rac1 and Arp2/3 as wells as transfected with the corresponding siRNAs and were analyzed regarding microtubule plus-end dynamics, spindle axis alignment and lagging chromosomes. The used inhibitors, NSC23766 and CK666 are established inhibitors known for their ability to block Rac1 activation by TRIO and TIAM1 (Gao, Dickerson, Guo, Zheng, \& Zheng, 2004) and inhibition of the Arp2/3 complex (Nolen et al., 2009), respectively. RhoA inhibition can be achieved by the addition of Clostridium botulinum exoenzyme C3 protein, which was shown to be responsible for ADP-ribosylation of Rho, thereby inhibiting activation of downstream effectors of RhoA (Genth et al., 2003).
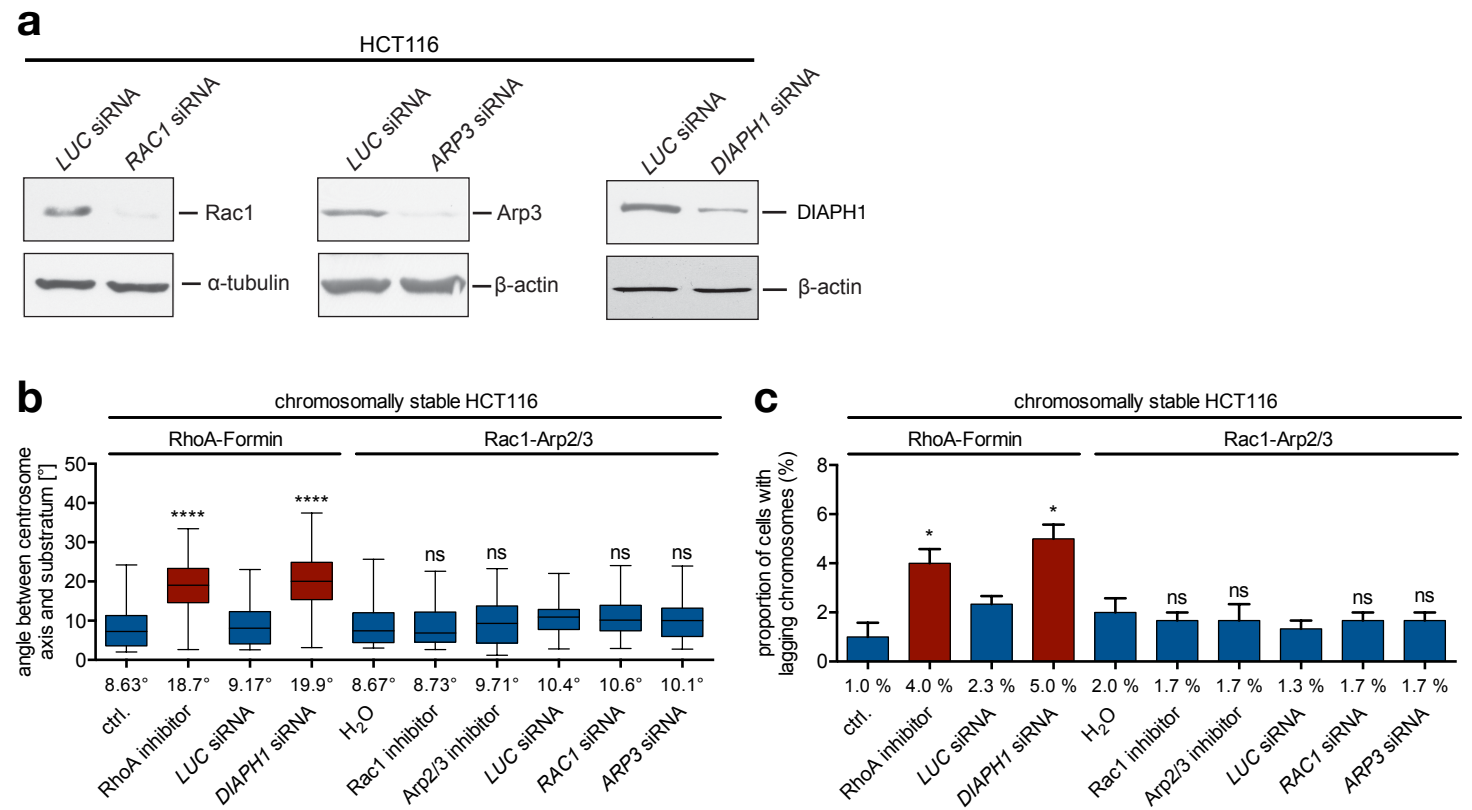

Fig. 3.15 In contrast to inhibition of the Rac1-Arp2/3 pathway, suppression of RhoA signaling induces spindle axis misalignment and the generation of lagging chromosomes in chromosomally stable HCT116 cells.

(a) Partial depletion of RAC1, ARP3 and DIAPH1 in HCT116 cells after siRNA transfection was confirmed by western blot analyses. $\alpha$-tubulin and $\beta$-actin were used as a loading control. (b) Spindle axis alignment in chromosomally stable HCT116 cells in prometaphase after repression of DIAPH1, RAC1 and ARP3. As a control, cells were transfected with LUCIFERASE siRNA (LUC). For This purpose, cells were transfected with siRNAs $48 \mathrm{~h}$ prior fixation. Inhibitor treatment was performed by the addition of a RhoA inhibitor ( 3 $\mu \mathrm{g} / \mathrm{ml}$ Native Clostridium botulinum Exoenzyme C3 protein) $6 \mathrm{~h}$ prior fixation. Additionally, Rac1 (40 $\mu \mathrm{M}$ NSC23766) and Arp2/3 (20 $\mu \mathrm{M}$ CK666) inhibitors were added $16 \mathrm{~h}$ prior fixation. For immunofluorescence microscopy, cells were grown on fibronectin coated fixed and stained for a-tubulin and $y$-tubulin. The box and whisker diagram show the median (mean values were added), the 25th to 75th percentile and the minimum and maximum values ( $n=3,60$ cells analyzed in total, $t$-test). (c) Quantification of lagging chromosomes in Rac1, Arp2/3 or RhoA inhibited HCT116 cells. Cells were transfected as described in (b). For synchronization at G1/S-phase, a double thymidine block was performed, and inhibitors were added for $6 \mathrm{~h}$ (RhoA inhibitor) or $8 \mathrm{~h}$ (Rac1 and Arp2/3 inhibitor). After releasing the cells into the cell cycle for $8.5 \mathrm{~h}$, 
cells were fixed and stained for immunofluorescence microscopy. Colocalization of CENP-C and chromatids lagging behind in anaphase are display by bar graphs $(n=3$, total amount of 300 analyzed anaphases, mean values \pm SEM, $t$-test). Indicated $p$-values refer to corresponding control cells. ns $=$ not significant, $p>0.05$; ${ }^{*}: p \leq 0.05 ;{ }^{* * * *}: \leq 0.0001$

Western blot analysis verified partial depletion of Rac1, Arp3 and DIAPH1 (Fig. 3.16a). Interestingly, only inhibition of RhoA but not Rac1 or Arp2/3 had an effect on HCT116 cells. After inhibition of RhoA by native Clostridium botulinum exoenzyme C3 protein or repression of DIAPH1, the angle of spindle axis alignment in HCT116 cells was increased from $8.63^{\circ}$ to $18.7^{\circ}$ and from $9.17^{\circ}$ to $19.9^{\circ}$, respectively (Fig. 3.15b). Inhibition of Rac1 or Arp2/3 did not change spindle axis alignment in HCT116 cells (Fig. 3.15b). Regarding chromosome mis-segregation, RhoA inhibition resulted in a four-fold increase of cells with lagging chromosomes in HCT116 cells (Fig. 3.15c). Likewise, partial depletion of DIAPH1 resulted in the generation of lagging chromosomes (Fig. 3.15c). However, inhibition of the Rac1-Arp2/3 pathway by either inhibitors or after siRNA transfection had no effect on chromosome segregation in HCT116 cells (Fig. 3.15c).

a
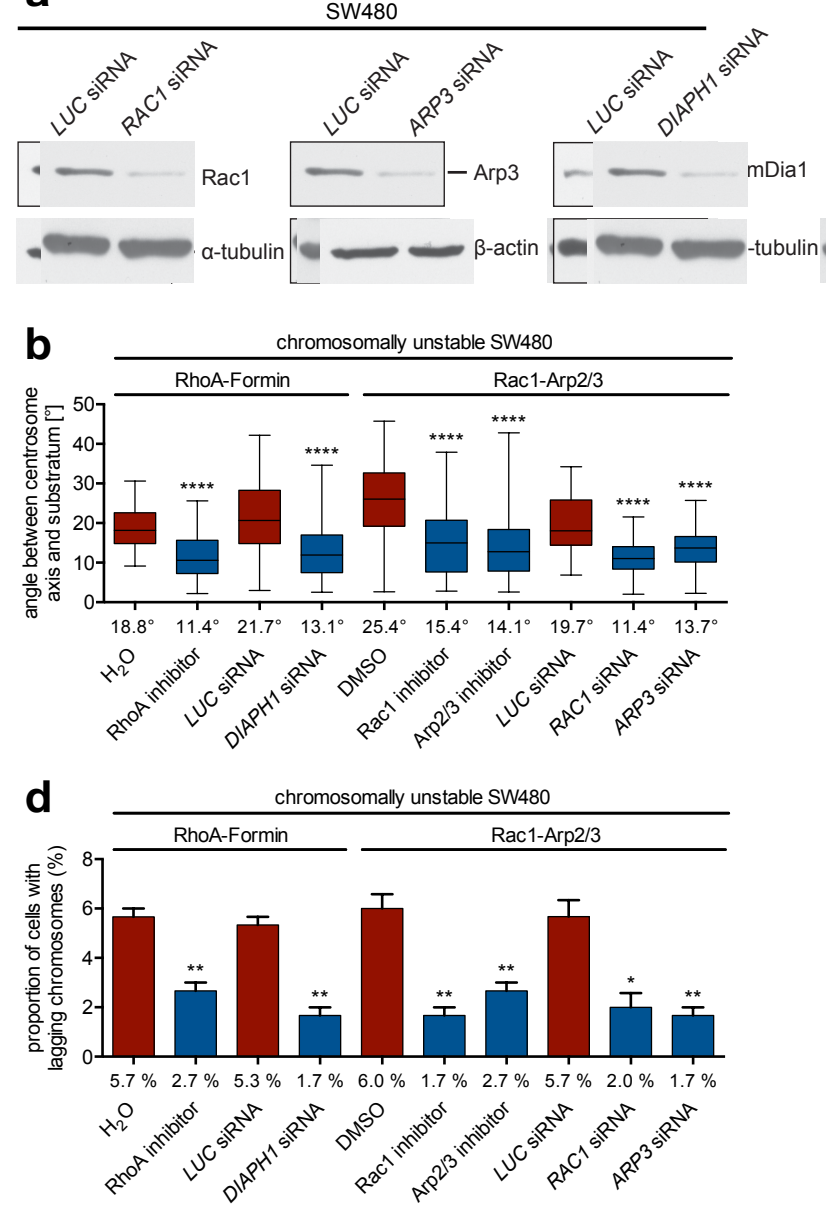

SW620
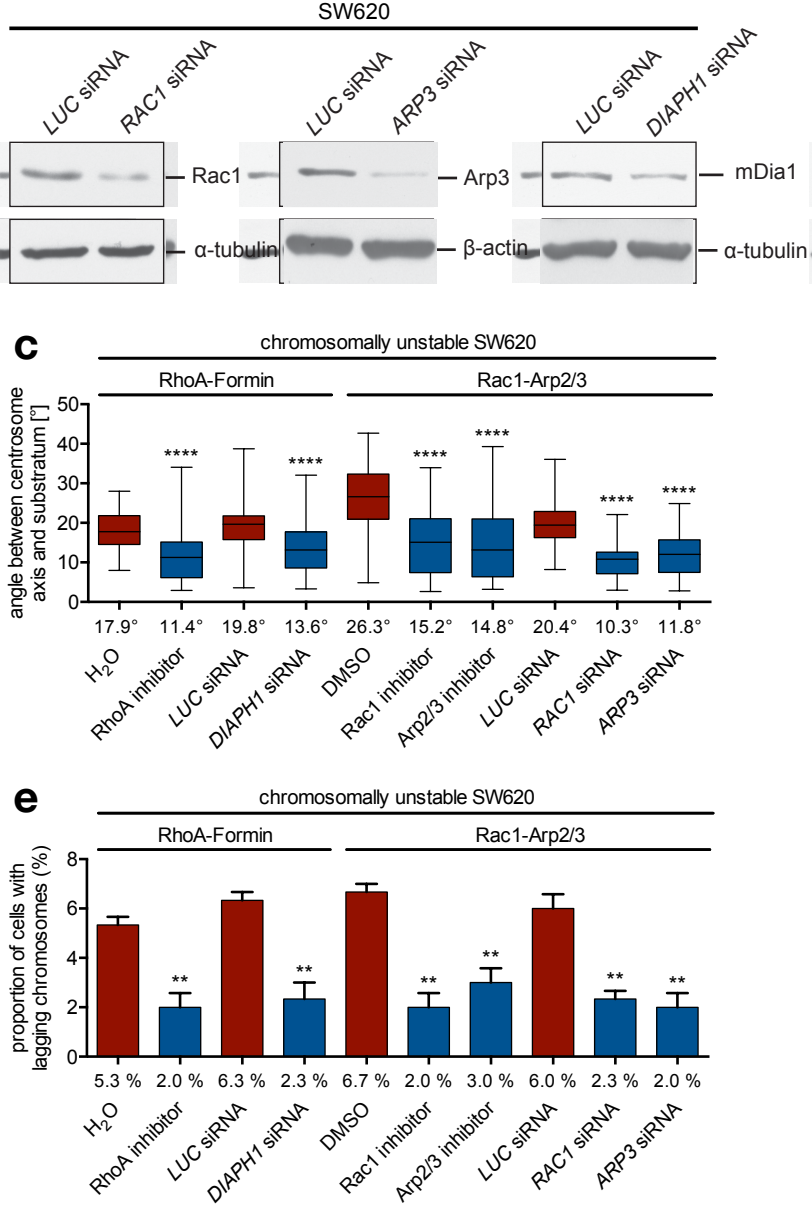
Fig. 3.16 Inhibition of Rho signaling and repression of the Rac1-Arp2/3 pathway restores proper spindle axis alignment in prometaphase and chromosome segregation in chromosomally unstable cells.

(e) Western blot analysis was used to verify partial depletion of RAC1, ARP3 and DIAPH1 in SW480 cells and SW620 cells after siRNA transfection. $\alpha$-tubulin and $\beta$-actin were used as a loading control. (b-c) Measurement of spindle axis alignment in SW480 cells (b) and SW620 cells (c) during prometaphase. To inhibit RhoA or Rac1-Arp2/3 signaling, cells were transfected with either LUCIFERASE (LUC) siRNA as a control or DIAPH1 siRNA, RAC1 siRNA or ARP3 siRNA. Moreover, RhoA inhibitor $(3 \mu \mathrm{g} / \mathrm{ml}$ Native Clostridium botulinum Exoenzyme C3 protein) was added $6 \mathrm{~h}$ prior fixation while Rac1 (40 $\mu \mathrm{M}$ NSC23766) and Arp2/3 (20 $\mu \mathrm{M}$ CK666) were added $16 \mathrm{~h}$ prior fixation. Cells were grown on fibronectin coated coverslips and were fixed and stained for immunofluorescence microscopy. The box and whisker diagram show the median (mean values were added), the 25th to 75 th percentile and the minimum and maximum values $(n=3$, 60 cells analyzed in total, $t$-test). (d-e) Quantification of lagging chromosomes in SW480 cells (d) and SW620 cells (e). Cells were transfected as described in (b-c). For accumulation of cells in anaphase, cells were synchronized at G1/S-phase by a double thymidine block. After releasing the cells into the cell cycle

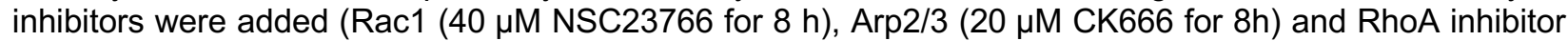
$(3 \mathrm{\mu g} / \mathrm{ml}$ Native Clostridium botulinum Exoenzyme C3 protein for $6 \mathrm{~h}$ ). Following $8.5 \mathrm{~h}$ of release, cells were fixed and stained for immunofluorescence microscopy. Bar graphs display the amount of cells with a CENP$C$ positive chromatid lagging behind in anaphase $(n=3$, total amount of 300 analyzed anaphases, mean values \pm SEM, $t$-test). Indicated $p$-values refer to corresponding control cells. ${ }^{*}: p \leq 0.05 ;{ }^{* *}: p \leq 0.01 ;{ }^{* * * *}$ $: p \leq 0.0001$

Intriguingly, in SW480 cells and SW620 cells, inhibition of RhoA as well as repression of the Rac1Arp2/3 pathway, which was confirmed by western blot analysis (Fig. 3.16a), restored proper spindle axis alignment in prometaphase and reduced the proportion of cells with lagging chromosomes (Fig. 3.16b-c). In SW480 cells, RhoA inhibition by C3 toxin and partial depletion of DIAPH1 decreased the angle of spindle axis alignment from $18.8^{\circ}$ to $11.4^{\circ}$ and from $21.7^{\circ}$ to $13.1^{\circ}$, respectively (Fig. 3.16b). Additionally, repression of Rac1-Arp2/3 signaling after siRNA transfection or inhibitor addition resulted in restored spindle axis alignment in SW480 cells during prometaphase (Fig. 3.16b). Similarly, in SW620 cells proper spindle axis alignment in prometaphase was restored upon inhibition of Rho and Rac1-Arp2/3 signaling (Fig. 3.16c). Moreover, in SW480 cells and SW620 cells, the proportion of cells with lagging chromosomes was reduced by almost two-thirds after inhibition of RhoA or the Rac1-Arp2/3 pathway (Fig. 3.16d-e). Regarding the proportion of cells with lagging chromosomes in anaphase, it was previously reported that inhibition of the Rac1-Arp2/3 pathway reduced chromosome mis-segregation in SW620 cells while the amount of cells with lagging chromosomes in HCT116 cells was not affected (Berger, 2016).

Given the fact that inhibition of RhoA and DIAPH1 influenced both, chromosomally stable and unstable cells, it is likely that a RhoA-formin pathway is active in chromosomally stable and unstable cells, which ensures proper spindle orientation based on unbranched actin polymerization thereby promoting proper cortical tension. In contrast, TRIO-Rac1-Arp2/3 signaling might be additionally active in CIN cells since inhibition of this pathway had no influence on 
HCT166 cells while it restored proper spindle axis alignment in SW480 cells and SW620 cells. Thus, these data indicate that Arp2/3 mediated branched actin polymerization leads to actin deregulation specifically in CIN cells, which results in reduced cortical tension thereby facilitating spindle axis misalignment.
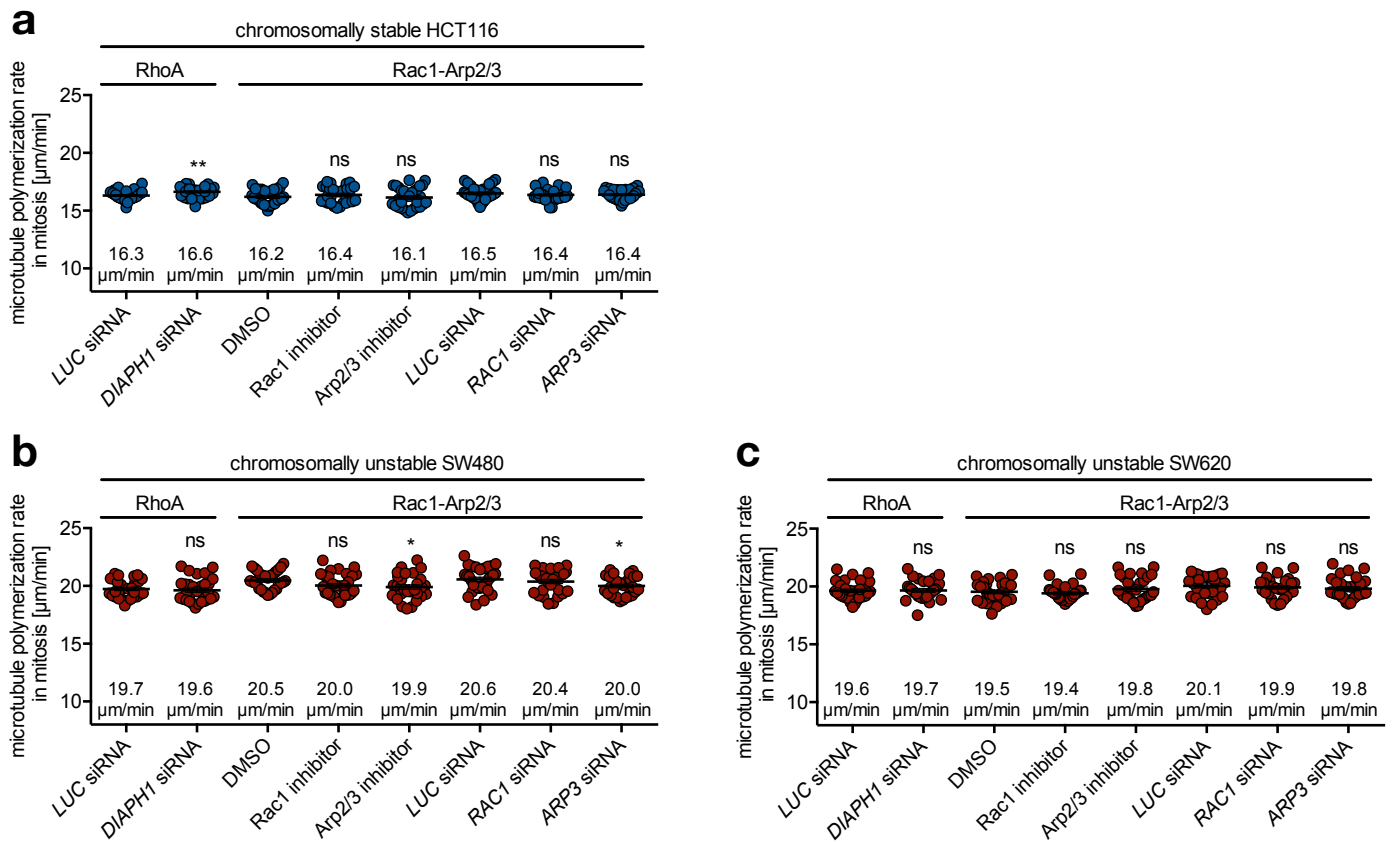

Fig. 3.17 DIAPH1 and the Rac-Arp2/3 pathway have no influence on microtubule plus-end assembly rates.

(a-c) Microtubule plus-end assembly rates in HCT116 cells (a), SW480 cells (b) and SW620 cells (c) during mitosis after inhibition of RhoA or Rac1-Arp2/3 signaling. Therefore, cells were transfected $48 \mathrm{~h}$ prior measurement with either LUCIFERASE (LUC) siRNA as a control or DIAPH1 siRNA, RAC1 siRNA or ARP3 siRNA. For EB3 tracking, transfected cells were incubated with $2 \mu \mathrm{M}$ DME for $1 \mathrm{~h}$ (HCT116 cells) or $1.5 \mathrm{~h}$ (SW480 cells and SW620 cells) or cells were treated simultaneously with inhibitors for Rac1 (40 $\mu \mathrm{M}$ NSC23766) and Arp2/3 (20 $\mu$ MCK6666). Scatter dot plots show mean values \pm SEM of 20 microtubules analyzed per cell of three independent experiments (30 cells analyzed in total, $t$-test). Indicated $p$-values refer to corresponding control cells. ns $=$ not significant, $p>0.05 ;{ }^{*}: p \leq 0.05$;

In order to investigate whether DIPAH1 and the Rac1-Arp2/3 pathway act down- or upstream of microtubule dynamics, HCT116 cells as well as SW480 cells and SW620 cells were analyzed after partial depletion of DIAPH1, Rac1 and Arp3. Additionally, inhibitors for Rac1 and Arp2/3 were used and microtubule plus-end assembly rates were measured. Neither inhibitor treatment nor repression of DIAPH1, RAC1 or ARP3 had an impact on microtubule dynamics in HCT116 cells since microtubule plus-end assembly rates are barely changed ranging from $16.1 \mu \mathrm{m} / \mathrm{min}$ to $16.5 \mu \mathrm{m} / \mathrm{min}$ (Fig. 3.17a). Similarly, in SW480 cells and SW620 cells microtubule plus-end assembly rates were not affected by inhibition of Rac1, Arp2/3 or DIAPH1 (Fig. 3.17b-c). These data suggest, that these proteins act downstream of microtubule plus-end assembly rates and 
thus, actin polymerization mediated by DIAPH1 or Rac1and Arp2/3 has no effect on microtubule dynamics. Moreover, these findings are in line with previous results that already demonstrated that the Rac1-Arp2/3 pathway had no impact on microtubule plus-end assembly rates (Berger, 2016).

To further strengthen the role of formins for spindle axis alignment in prometaphase, cells were cultivated in the presence of a formin inhibitor (SMIFH2), which inhibits the FH2 domain of several formins thereby preventing formin mediated actin nucleation (Rizvi et al., 2009). Like inhibition of RhoA signaling by native Clostridium botulinum exoenzyme C3 protein or repression of DIAPH1, inhibition of formins led to an induction of spindle axis misalignment in chromosomally stable HCT116 cells and RKO cells while it restored proper spindle axis alignment in chromosomally unstable SW480 cells and SW620 cells. In HCT116 cells the angle increased from $9.56^{\circ}$ to $17.6^{\circ}$ while in RKO cells the spindle angle was increased two-fold (Fig. 3.18a).
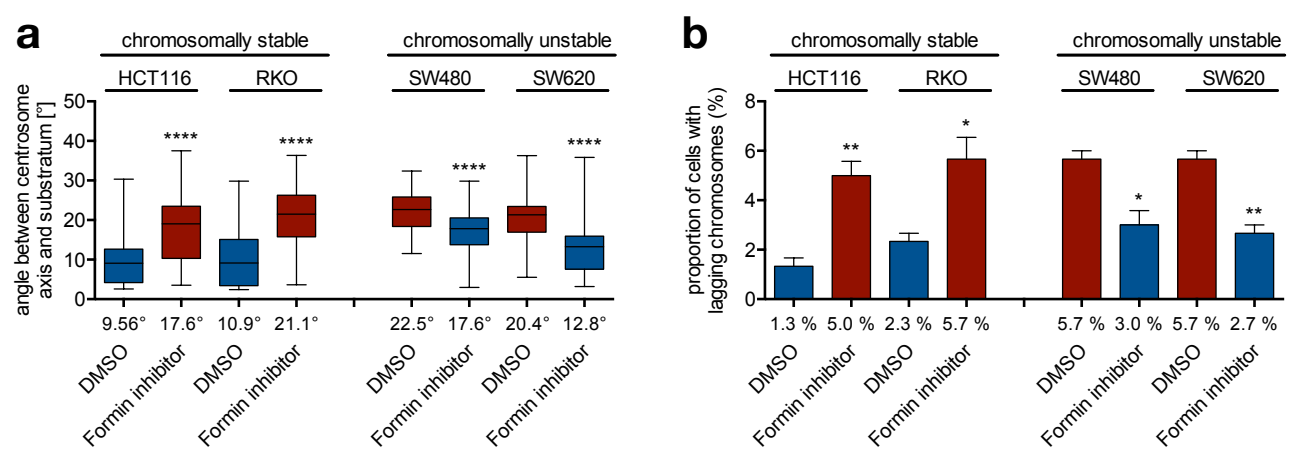

Fig. 3.18 Formin inhibition restores proper spindle axis alignment and chromosome segregation in CIN cells whereas it promotes chromosomes mis-segregation and spindle axis misalignment in MIN/MSI cells.

(a) Spindle axis alignment in chromosomally stable HCT116 cells and RKO cells as well as chromosomally unstable SW480 cells and SW620 cells during prometaphase after formin inhibition. Cells were seeded on fibronectin coated coverslips and $20 \mu \mathrm{M}$ of the formin inhibitor SMIFH2 was added $2 \mathrm{~h}$ prior fixation. Subsequently, cells were fixed and stained for immunofluorescence microscopy. The box and whisker diagram show the median (mean values were added), the 25th to 75th percentile and the minimum and maximum values ( $n=3,60$ cells analyzed in total, $t$-test). (b) Analysis of lagging chromosomes after formin inhibition. For this purpose, a double thymidine block was performed to synchronize cells at G1/S-phase and released into the cell cycle for $8.5 \mathrm{~h}$. $2 \mathrm{~h}$ prior fixation, $20 \mu \mathrm{M}$ of the formin inhibitor SMIFH2 was added. Afterwards, cells were fixed and stained for immunofluorescence microscopy. Bar graphs displays the amount of cells with a significantly delayed CENP-C positive chromatid in anaphase $(n=3$, total amount of 300 analyzed anaphases, mean values \pm SEM, $t$-test). ${ }^{*}: p \leq 0.05 ;{ }^{* *}: p \leq 0.01 ;{ }^{* * * *}: p \leq 0.0001$

Control SW80 cells and SW620 cells exhibited an average angle of $21.5^{\circ}$, which was reduced to an average of $15.2^{\circ}$ upon inhibitor addition (Fig. 3.18a). Similarly, formin inhibition resulted in chromosome mis-segregation in HCT116 cells and RKO cells. Here, the average proportion of cells with lagging chromosomes significantly increased from $1.8 \%$ to $5.4 \%$ (Fig. 3.18b) whereas 
in SW80 cells and SW620 cells the average number of cells with lagging chromosomes decreased from $5.7 \%$ to $2.9 \%$ after preventing formin mediated actin nucleation (Fig. 3.18b).

These results indicate, that an actin network, composed of a specific actin filament structure is required to ensure proper spindle axis alignment, which might be achieved by RhoA-formin mediated unbranched actin nucleation in chromosomally stable and unstable cells. Thus, it seems possible that additional actin polymerization based on the Rac1-Arp2/3 pathway results in reduced cortex tension thereby leading to spindle axis misalignment in CIN cells. That's why it was of interest to analyze if enhanced actin nucleation promotes spindle axis misalignment in HCT116 cells.

To increase unbranched actin polymerization, either RHOA wildtype (RHOA-WT) or a constitutive active form (RHOA-CA) was overexpressed, which was verified by western blot analysis (Fig. 3.19a). Moreover, Calpeptin was used, which is a calpain inhibitor that also inhibits the Shp2 phosphatase thereby activating RhoA (Schoenwaelder et al., 2000). Interestingly, only overexpression of constitutive active but not wildtype RHOA was able to induce spindle axis misalignment during prometaphase in HCT116 cells (Fig. 3.19b). The addition of calpeptin had a similar effect on spindle axis alignment and resulted in a two-fold increase of the spindle angle (Fig. 3.19a). Intriguingly, activation of RhoA by calpeptin did not influence spindle axis alignment in chromosomally unstable SW480 cells and SW620 cells (Fig. 3.19c). In terms of chromosome segregation, control HCT116 cells revealed $1.9 \%$ of cells with lagging chromosomes while RhoA activation resulted in $5.3 \%$ of cells exhibiting lagging chromosomes (Fig. 3.19d). As observed for spindle axis misalignment in SW480 and SW620 cells, calpeptin treatment did not change the amount of cells with lagging chromosomes (Fig. 3.19e).

These results indicate that increased unbranched actin nucleation mediated by RhoA promotes spindle axis misalignment in chromosomally stable cells and thus, further strengthen the hypothesis that enhanced actin polymerization is the cause for spindle axis misalignment in CIN cells.

Since proper spindle axis misalignment in CIN cells was restored upon formin and Arp2/3 inhibition, it was of interest to analyze if this inhibition is also sufficient to restore proper spindle axis alignment after the induction of spindle axis misalignment in chromosomally stable HCT116 cells. For this purpose, RHOA-CA was overexpressed in HCT116 cells. Additionally, transfected cells were treated with inhibitors for the Arp2/3 complex and $\mathrm{FH} 2$ domain of formins. Subsequently, spindle axis alignment as well as the proportion of cells with lagging chromosomes was investigated. 

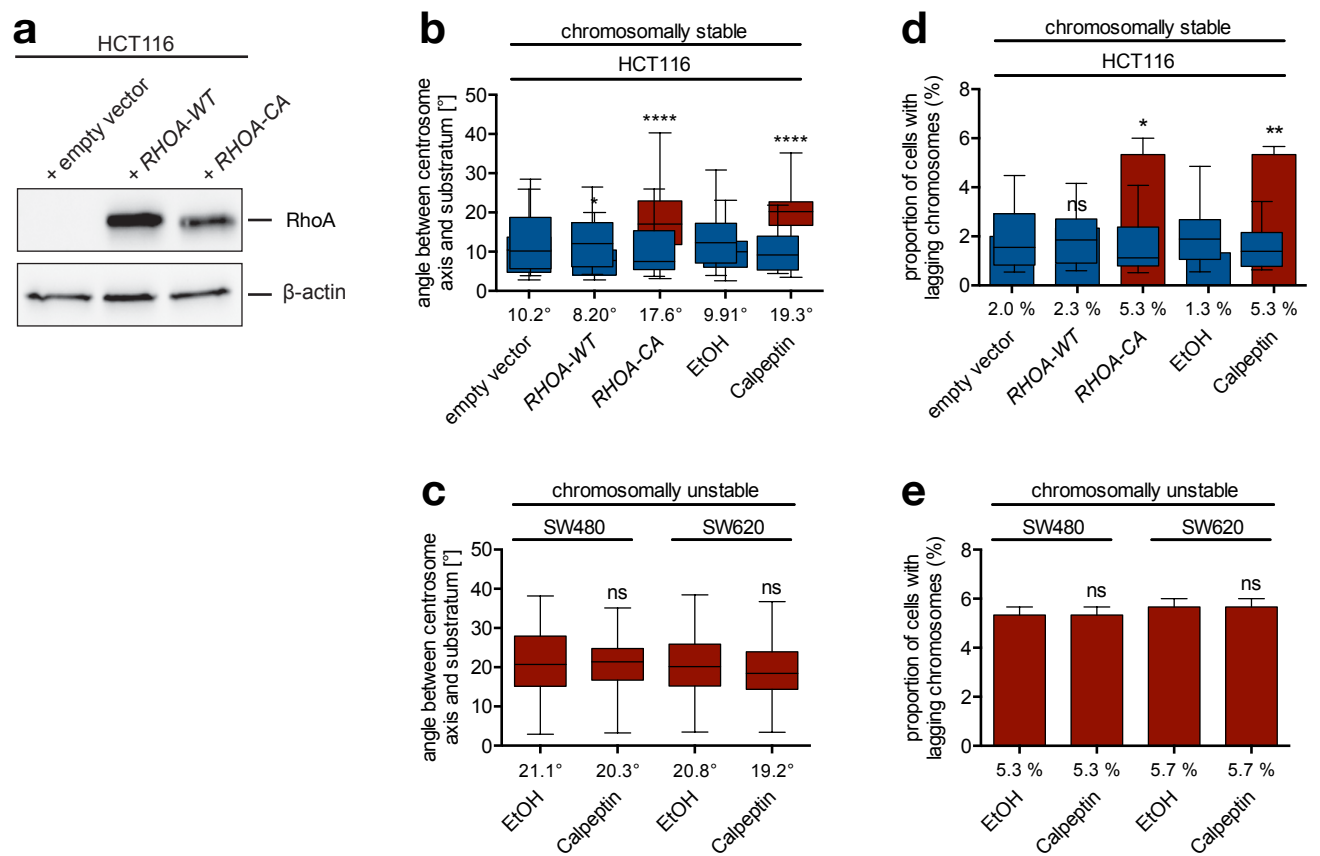

Fig. 3.19 RhoA activation leads to spindle axis misalignment and chromosome mis-segregation in chromosomally stable HCT116 cells but has no effect on CIN cells.

(a) Representative western blot showing an overexpression of RHOA-WT and RHOA-CA in HCT116 cells. $\beta$-actin was used as a loading control. (b-c) Analysis of the angle between the centrosome axis and substratum in chromosomally stable HCT116 cells (b), chromosomally unstable SW480 cells and SW620 cells (c) in prometaphase after activation of RhoA by either overexpression of RHOA or addition of Calpeptin. Therefore, HCT116 cells were transfected with either RHOA-WT or RHOA-CA $48 \mathrm{~h}$ prior analysis or treated with $1 \mu \mathrm{g} / \mathrm{ml}$ Calpeptin or EtOH as a control for $6 \mathrm{~h}$. Cells were seeded on fibronectin coated coverslips and were fixed and stained for immunofluorescence microscopy. The box and whisker diagram show the median (mean values were added), the 25th to 75th percentile and the minimum and maximum values $(n=3,60$ cells analyzed in total, $t$-test). (d-e) Quantification of lagging chromosomes in HCT116 cells (d), SW480 cells and SW620 cells (e). Cells were transfected and treated with calpeptin as described in (b). To analyze lagging chromosomes, cells were synchronized at G1/S-phase by a double thymidine block and released into the cell cycle for $8.5 \mathrm{~h}$. Subsequently, cells were fixed and stained for immunofluorescence microscopy. Bar graphs show the proportion of cells exhibiting the amount of cells with lagging chromosomes ( $n=3$, total amount of 300 analyzed anaphases, mean values \pm SEM, $t$-test). Indicated $p$-values refer to corresponding control cells. ns $=$ not significant, $p>0.05 ;{ }^{*}: p \leq 0.05 ;{ }^{* *}: p \leq 0.01 ;{ }^{* * *}: p \leq 0.0001$

As observed before, overexpression of RHOA-CA led to a two-fold increase of the spindle angle in HCT116 cells (Fig. 3.20a). Remarkably, spindle axis misalignment induced by overexpression of constitutive active RHOA was only reversed after addition of the formin inhibitor but not after inhibition of Arp2/3 (Fig. 3.20a). The same effect was observed regarding the amount of cells with lagging chromosomes. Here, an overexpression of constitutive active RHOA increased the proportion of cells with lagging chromosomes from $1.7 \%$ to $5.0 \%$ (Fig. 3.20b), which was reduced upon formin inhibition to $2.0 \%$ while Arp2/3 inhibition had no impact on chromosome segregation (Fig. 3.20b). 

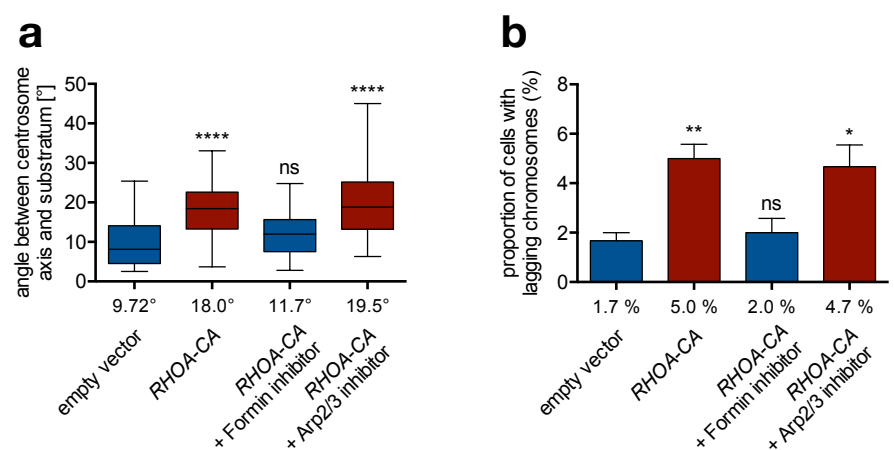

Fig. 3.20 Spindle axis misalignment induced by RhoA activation is only restored by formin inhibition but not by Arp2/3 inhibition.

(a) Spindle axis alignment in chromosomally stable HCT116 cells after overexpression of RHOA-CA and additional formin and Arp2/3 inhibition. HCT166 cells were transfected $48 \mathrm{~h}$ before fixation with RHOA-CA and additionally treated with $20 \mu \mathrm{M} \mathrm{SMIFH} 2$ (formin inhibitor) for $2 \mathrm{~h}$ or with $20 \mu \mathrm{M} \mathrm{CK666} \mathrm{(Arp2/3} \mathrm{inhibitor)}$ for $16 \mathrm{~h}$. For analyzing spindle axis alignment, cells were grown on fibronectin coated coverslips fixed and stained for immunofluorescence microscopy. The box and whisker diagram show the median (mean values values were added), the 25th to 75th percentile and the minimum and maximum values $(n=3,60$ cells analyzed in total, $t$-test). (b) Detection of proportion of cells exhibiting lagging chromosomes in HCT116 cells. Cells were transfected as described in (a). To accumulate anaphases, a double thymidine block was performed to synchronize cells at G1/S-phase. After releasing the cells into the cell cycle, $20 \mu \mathrm{M}$ CK666 (Arp2/3 inhibitor) was added for $8 \mathrm{~h}$ or $20 \mu \mathrm{M}$ of SMIFH2 (formin inhibitor) was added for $2 \mathrm{~h}$. After $8.5 \mathrm{~h}$, cells were fixed and stained for immunofluorescence microscopy. Bar graphs show the proportion of cells with a CENP-C positive lagging chromatid $(n=3$, total amount of 300 analyzed anaphases, mean values \pm SEM, $t$-test). ns $=$ not significant, $p>0.05 ;{ }^{*}: p \leq 0.05 ;{ }^{* *}: p \leq 0.01 ;{ }^{* * *}: p \leq 0.0001$

Taken together, enhanced unbranched actin polymerization resulted in spindle axis misalignment in HCT116 cells, which was only restored after inhibition of formins but not Arp2/3, further indicating that proper spindle orientation requires a specific actin cortex structure. However, these results suggest that, regardless of Arp2/3 or formin mediated actin polymerization, increased actin polymerization in general promotes spindle axis misalignment.

\subsection{A Rac1-Arp2/3 pathway is responsible for spindle axis misalignment in chromosomally unstable cells downstream of microtubule plus-end assembly rates}

Initial experiments from our lab already revealed that a hyperactive Rac1-Arp2/3 pathway is implicated in spindle axis misalignment in CIN cells (Berger, 2016). Together with the observation that increased unbranched actin polymerization induced spindle axis misalignment, it was of interest to analyze if hyperactive Rac1-Arp2/3 signaling and the resulting branched actin polymerization is sufficient to induce spindle axis misalignment in chromosomally stable HCT116 cells. Moreover, additional experiments were performed to analyze the exact order of Rac1 and Arp2/3 signaling. Therefore, HCT116 cells were transfected with a constitutive active form of Rac1 (Rac1-CA) and additionally incubated with the Arp2/3 inhibitor or with Taxol. Subsequently, spindle axis alignment and chromosome segregation were analyzed. Overexpression of RAC1-CA was 
confirmed by western blot analysis (Fig. 3.21a) and had no impact on microtubule plus-end assembly rates (Fig. 3.21b).
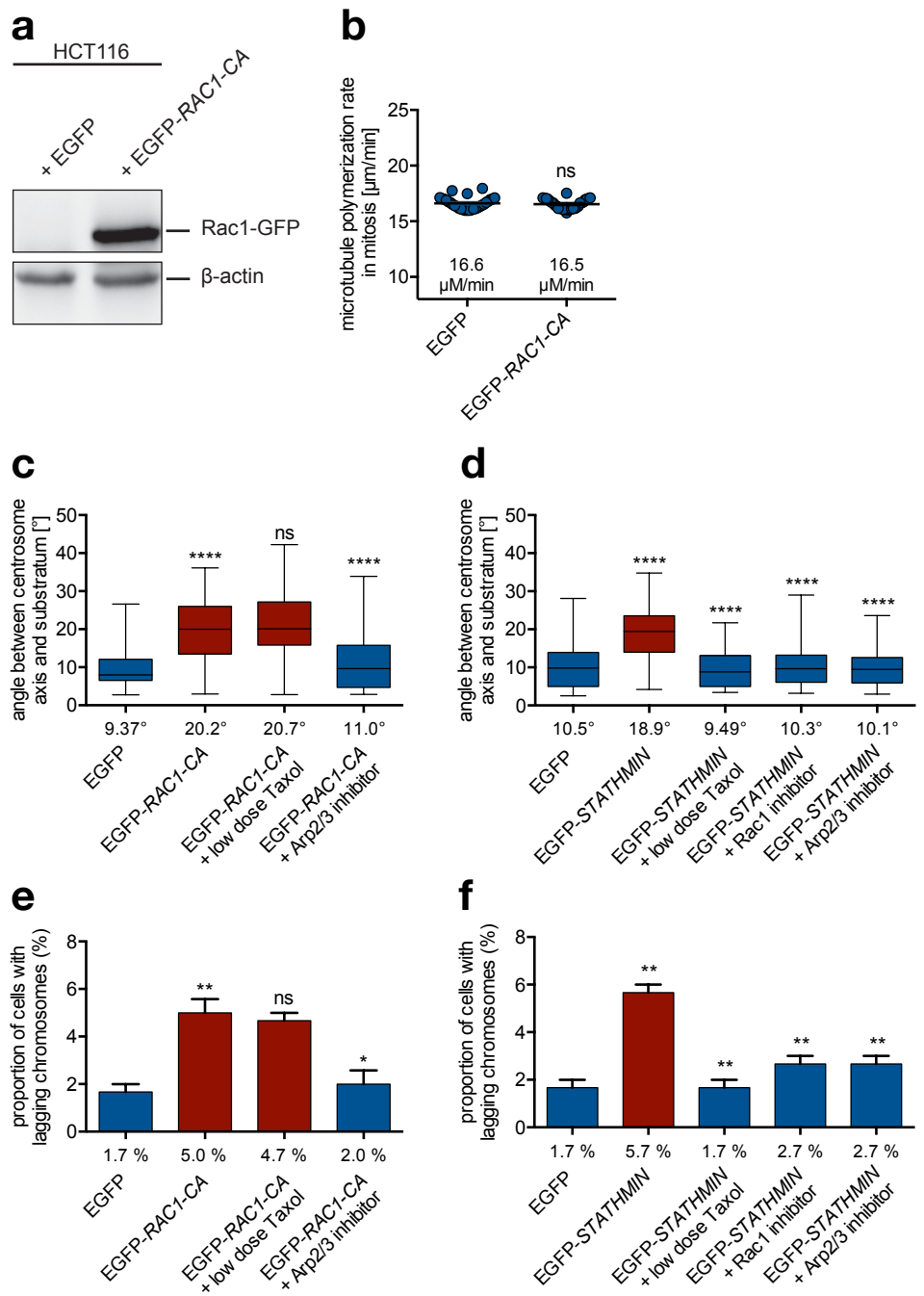

Fig. 3.21 The Rac1-Arp2/3 pathway is responsible for spindle axis misalignment and chromosome mis-segregation downstream of microtubule plus-end dynamics.

(a) Representative western blot confirming an overexpression of RAC1-CA in HCT116 cells. $\beta$-actin was used as a loading control. (b) Microtubule plus-end assembly rates in mitotic HCT116 cells overexpressing RAC1-CA. For EB3 tracking, cells were transfected with RAC1-CA and treated with $2 \mu \mathrm{M}$ DME $1 \mathrm{~h}$ before measurements. (c-d) Spindle axis alignment in chromosomally stable HCT116 cells after RAC1-CA (c) or STATHMIN (d) overexpression. Therefore, cells were transfected with RAC1-CA or STATHMIN $48 \mathrm{~h}$ prior fixation. For analyzing spindle angles, transfected cells seeded on fibronectin coated coverslips and were grown for $16 \mathrm{~h}$ in medium containing $0.2 \mathrm{nM}$ Taxol, $40 \mu \mathrm{M}$ NSC23766 (Rac1 inhibitor) or $20 \mu \mathrm{M}$ CK666 (Arp2/3 inhibitor). Afterwards, cells were fixed and stained for immunofluorescence microscopy. The box and whisker diagram show the median (mean values were added), the 25th to 75th percentile and the minimum and maximum values ( $\mathrm{n}=3,60$ cells analyzed in total, $t$-test). (e-f) Quantification of lagging chromosomes in HCT116 overexpressing RAC1-CA (e) or STATHMIN (f). Cells were transfected as described in (c-d) and were synchronized at G1/S-phase by a double thymidine block. Cells were released into the cell cycle in the presence of $0.2 \mathrm{nM}$ Taxol, $40 \mu \mathrm{M}$ NSC23766 (Rac1 inhibitor) or $20 \mu \mathrm{M}$ CK666 (Arp2/3 inhibitor). After $8.5 \mathrm{~h}$, cells were fixed and stained for immunofluorescence microscopy. Bar graphs 
show the proportion of cells exhibiting lagging chromosomes $(n=3$, total amount of 300 analyzed anaphases, mean values \pm SEM, $t$-test $)$. $n s=$ not significant, $p>0.05 ;{ }^{*}: p \leq 0.05 ;{ }^{* *}: p \leq 0.01 ;{ }^{* * * *}: p \leq 0.0001$

Intriguingly, the spindle angle in HCT116 cells was increased two-fold upon overexpression of RAC1-CA (Fig. 3.21c), whereas only inhibition of the Arp2/3 complex but not Taxol treatment restored proper spindle axis alignment (Fig. 3.21c). Accordingly, RAC1-CA overexpression was sufficient to induce chromosome mis-segregation (Fig. 3.21e), which is in line with previous findings (Berger, 2016). Moreover, only Arp2/3 inhibition reduced the amount of cells with lagging chromosomes induced by RAC1-CA overexpression from $5 \%$ to $2 \%$ (Fig. 3.21e). Thus, these results indicate that spindle axis misalignment is induced by RAC1-CA downstream of microtubule dynamics. Next, HCT116 cells were transfected with STATHMIN and additionally treated with low doses of Taxol and inhibitors for Rac1 and Arp2/3. In contrast to RAC1-CA induced spindle axis misalignment, low doses of Taxol decreased the spindle angle after STATHMIN overexpression from $18.9^{\circ}$ to $9.49^{\circ}$ (Fig. 3.21d). Moreover, inhibition of Rac1 and Arp2/3 also reduced the spindle angle by almost half (Fig. 3.21d). Likewise, chromosome mis-segregation induced by STATHMIN overexpression in HCT116 cells was reduced upon treatment with low doses of Taxol and Rac1 or Arp2/3 inhibition since the amount of cells with lagging chromosomes was decreased from $5.7 \%$ to an average of $2.4 \%$ (Fig. 3.21f).

These findings suggest that Rac1 and Arp2/3 act downstream of microtubule plus-end assembly rates. Since it was shown that a hyperactive Rac1-Arp2/3 pathway is dependent on increased microtubules plus-end assembly rates in mitosis (Berger, 2016), it is likely that enhanced microtubule dynamics lead to actin deregulation based on the hyperactivation of Rac1 and Arp2/3, which promotes branched actin polymerization thereby leading to reduced cortical tension, which is finally responsible for spindle axis misalignment. This is supported by the fact that restoration of proper microtubule plus-end assembly rates by Taxol resulted in proper cortical tension and spindle axis alignment in CIN cells.

\subsection{TRIO - a link between the microtubule and actin cytoskeleton that mediates spindle axis misalignment in chromosomally unstable cells with enhanced microtubule plus- end assembly rates}

A link between microtubule plus-end assembly rates and Rac1 during mitosis was already identified by previous lab members (Berger, 2016). This work demonstrated that TRIO activates Rac1 in an EB1 dependent manner in response to enhanced microtubule dynamics (Berger, 2016). Additionally, it was reported that TRIO binds to EB1 in a complex with Nav1 (Neuron navigator 1) (Van Haren et al., 2014). Thus, it was of interest to investigate if EB1, Nav1 and 
especially TRIO have an impact on spindle axis alignment in prometaphase and the proportion of cells with lagging chromosomes. To address this question, EB1 as wells as Nav1 were partial depleted in HCT116 cells, SW480 cells and SW620 cells. Analysis of HCT116 cells revealed that downregulation of EB1 and NAV1, which was verified by western blot analysis (Fig. 3.22a-b), had no effect on axis spindle alignment in prometaphase and the proportion of cells with lagging chromosomes (Fig. 3.22c-d).
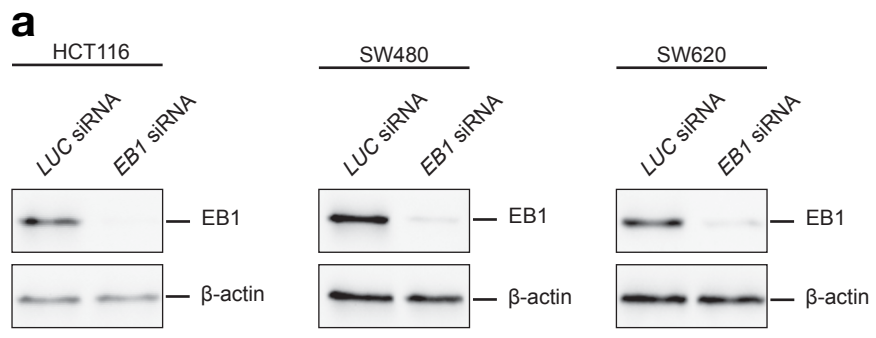

b
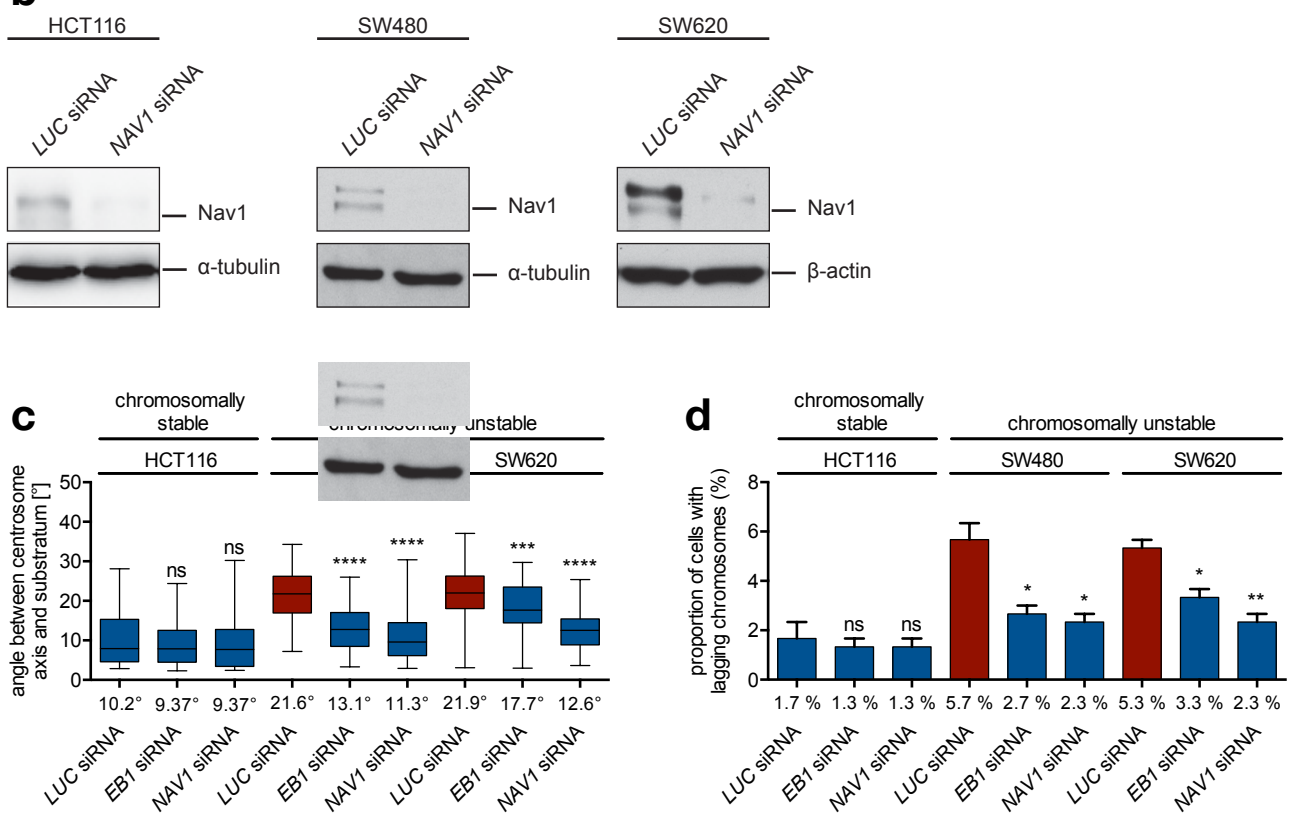

Fig. 3.22 Downregulation of NAV1 end EB1 restores proper spindle axis alignment prometaphase and lagging chromosomes in chromosomally unstable cells.

(a-b) Representative western blot verifying partial depletion of EB1 (a) and Nav1 (c) in HCT116 cells, SW480 cells and SW620 cells. $\beta$-actin was used as a loading control. (c) Analysis of the angle between the centrosome axis and substratum in chromosomally stable HCT116 cells as well as chromosomally unstable SW480 cells and SW620 cells after repression of EB1 and NAV1. For this purpose, LUCIFERASE (LUC) siRNA, EB1 siRNA or NAV1 siRNA transfected cells were seeded and grown on fibronectin coated coverslips. Afterwards, cells were fixed and stained for immunofluorescence microscopy. The box and whisker diagram show the median (mean values were added), the 25th to 75th percentile and the minimum and maximum values ( $n=3,60$ cells analyzed in total, $t$-test). (d) Analysis of lagging chromosomes in HCT116 cells, SW480 cells and SW620 cells after repression of EB1 or NAV1. Cells were transfected as described in (c) and synchronized at G1/S-phase by a double thymidine block. After releasing the cells into the cell cycle for $8.5 \mathrm{~h}$, cells were fixed and stained for immunofluorescence microscopy. Bar graphs displays the proportion of cells with lagging chromosomes $(n=3$, total amount of 300 analyzed anaphases, 
mean values \pm SEM, $t$-test). Indicated $p$-values refer to corresponding control cells. $n s=$ not significant, $p>$ $0.05 ;^{*}: p \leq 0.05 ;{ }^{* *}: p \leq 0.01 ;^{* * *}: p \leq 0.001 ;{ }^{* * * *}: p \leq 0.0001$

However, in SW480 cells the angle of spindle axis alignment was decreased from $21.6^{\circ}$ to $13.1^{\circ}$ and $11.3^{\circ}$ upon partial depletion of EB1 and Nav1, respectively (Fig. 3.22c). Likewise, the spindle angle in SW620 cells was almost reduced by half after repression of EB1 and NAV1 (Fig. 3.22c). Moreover, chromosome mis-segregation in SW480 cells and SW620 cells was also lowered since SW480 control cells exhibited an average of $5.7 \%$ of cells with lagging chromosomes, which was reduced to $2.7 \%$ while in SW620 cells the amount of lagging chromosomes was reduced by half (Fig. 3.22d). These results confirm that partial depletion of EB1 reduced the amount of cells with lagging chromosomes in SW620 cells as it was already shown (Berger, 2016). Next, the impact of partial depletion of TRIO on spindle axis alignment and chromosome segregation was analyzed. Therefore, HCT116 cells and SW480 cells as well as SW620 cells were transfected with TRIO siRNA or treated with a TRIO inhibitor (ITX3), which was shown to specifically block the interaction between TRIO and Rac1 thereby preventing Rac1 activity (Bouquier et al., 2009). First, repression of TRIO was confirmed by western blot analysis (Fig. 3.23a). In HCT116 cells TRIO inhibition or depletion had no effect on spindle axis alignment or the amount of cells with lagging chromosomes (Fig. 3.24b-c). However, these treatments were sufficient to restore proper spindle axis alignment in SW480 cells and SW620 cells. In SW480 cells the spindle angle was almost reduced by half (Fig. 3.24b) while in SW620 cells TRIO depletion or inhibition caused a decreased of the spindle angle from $21.5^{\circ}$ to $12.4^{\circ}$ and $16.1^{\circ}$, respectively (Fig. 3.24b). Likewise, inhibition of TRIO resulted in a reduced amount of cells with lagging chromosomes in SW480 cells and SW620 cells (Fig. 3.23c). Moreover, there was no effect on microtubule plus-end assembly rates in HCT116 cells, SW480 cells or SW620 cells upon repression of TRIO or inhibitor treatment (Fig. 3.23d). These data confirm previous findings since it was reported that addition of ITX3 restored proper spindle orientation and chromosome segregation in SW620 cells while inhibitor treatment had no impact on microtubule dynamics (Berger, 2016). These results indicate that TRIO, like Rac1 and Arp2/3, is only active in CIN cells since inhibition of TRIO did not affect MIN/MSI cells while it restored proper spindle axis alignment and chromosome segregation in CIN cells. Moreover, TRIO acts downstream of microtubule dynamics as it was observed for Rac1 and Arp2/3. Thus, the next step involved the overexpression of TRIO and its impact on spindle axis alignment and lagging chromosomes in chromosomally stable HCT116 cells. 
a
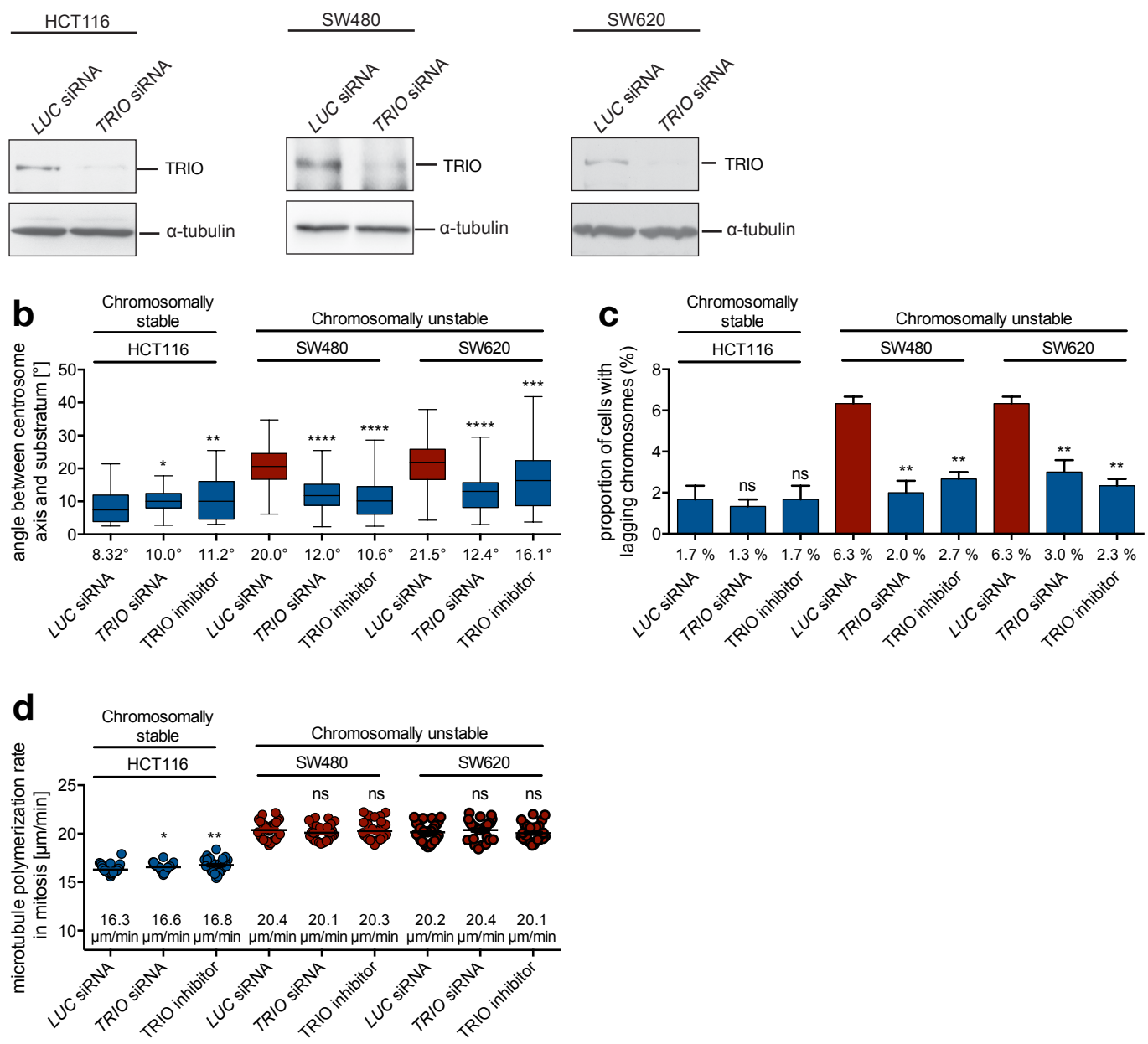

Fig. 3.23 Partial depletion or inhibition of TRIO restores proper spindle axis alignment and chromosome segregation in chromosomally unstable cells.

(a) Partial depletion of TRIO in HCT116 cells, SW480 cells and SW620 cells was confirmed by western blot analysis. $\beta$-actin was used as a loading control. (b) Analysis of spindle axis alignment in chromosomally stable HCT116 cells, chromosomally unstable SW480 cells and SW620 cells in prometaphase after partial depletion or inhibition of TRIO. Cells were transfected $48 \mathrm{~h}$ prior to fixation with either LUCIFERASE (LUC) siRNA or TRIO siRNA. For TRIO inhibition $7.5 \mu \mathrm{M}$ (SW480 cells) or $15 \mu \mathrm{M}$ (HCT116 cells and SW620 cells) ITX3 (TRIO inhibitor) was added $16 \mathrm{~h}$ prior fixation. For immunofluorescence microscopy cells were grown on fibronectin coated coverslips and fixed and stained with antibodies detecting a-tubulin and $\mathrm{y}$-tubulin. The box and whisker diagram show the median (mean values were added), the 25th to 75th percentile and the minimum and maximum values ( $n=3,60$ cells analyzed in total, $t$-test). (c) Analysis of lagging chromosomes in HCT116 cells, SW480 cells and SW620 cells after repression of TRIO or inhibitor treatment. To analyze anaphases, cells were transfected as described in (b) and synchronized at G1/S-phase by a double thymidine block. To inhibit, $15 \mu \mathrm{M}$ ITX3 was added at the time cells were released into the cell cycle. After $8.5 \mathrm{~h}$, cells were fixed and stained for immunofluorescence microscopy. Bar graphs show the proportion of cells exhibiting a colocalization a CENP-C positive chromatid lagging behind in anaphase $(n=3$, total amount of 300 analyzed anaphases, mean values \pm SEM, $t$-test). (d) Microtubule plus-end assembly rates of HCT116 cells, SW480 cells and SW620 cells in mitosis after TRIO inhibitor treatment or TRIO repression. For the latter cells were transfected as described in (b). To analyze EB3 dynamics, $2 \mu \mathrm{M}$ DME was added $1 \mathrm{~h}$ prior measurement and $15 \mu \mathrm{M}$ ITX3 was added at the same time for TRIO inhibition. Scatter dot plots show mean values \pm SEM of 20 microtubules analyzed per cell of three independent experiments (30 cells analyzed in total, $t$-test). Indicated $p$-values refer to corresponding control cells. $n s=$ not significant, $p>$ $0.05 ;^{*}: p \leq 0.05 ;{ }^{* *}: p \leq 0.01 ;^{* * *}: p \leq 0.001 ;^{* * * *}: p \leq 0.0001$ 
Since previous work showed that the ability of TRIO to activate Rac1 downstream of microtubule dynamics was dependent on its interaction with EB1 (Berger, 2016; Van Haren et al., 2014), besides wildtype TRIO, an EB1 binding deficient mutant (TRIO-SRNN) was also overexpressed. TRIO-SRNN harbors a mutation in the SxIP motif, which is responsible for EB1 binding and thus, prevents interaction between TRIO and EB1 (Van Haren et al., 2014). Additionally, TRIO-WT overexpressing HCT116 cells were treated with inhibitors for TRIO, Rac1 and Arp2/3. Importantly, overexpression of TRIO-WT in chromosomally stable HCT116 cells, which was confirmed by western blot analysis (Fig. 3.24a), resulted in spindle axis misalignment by increasing the spindle angle from $11 .^{\circ}$ to $20.6^{\circ}$ while HCT116 cells overexpressing the EB1 binding deficiency mutant did not exhibit altered spindle axis alignment (Fig. 3.24b). Interestingly, inhibitors for TRIO, Rac1 and Arp2/3 were able to restore proper spindle axis alignment in prometaphase by reducing the average spindle angle from $20.6^{\circ}$ to $10.5^{\circ}$ (Fig. 3.24b). Likewise, only overexpression of TRIOWT promoted the generation of lagging chromosomes in HCT116 cells whereas TRIO-SRNN overexpression had no effect on lagging chromosomes in anaphase (Fig. 3.24c). Moreover, inhibition of TRIO, Rac1 and Arp2/3 was sufficient to reduce the amount of cells with lagging chromosomes induced by wildtype TRIO overexpression (Fig. 3.24c)
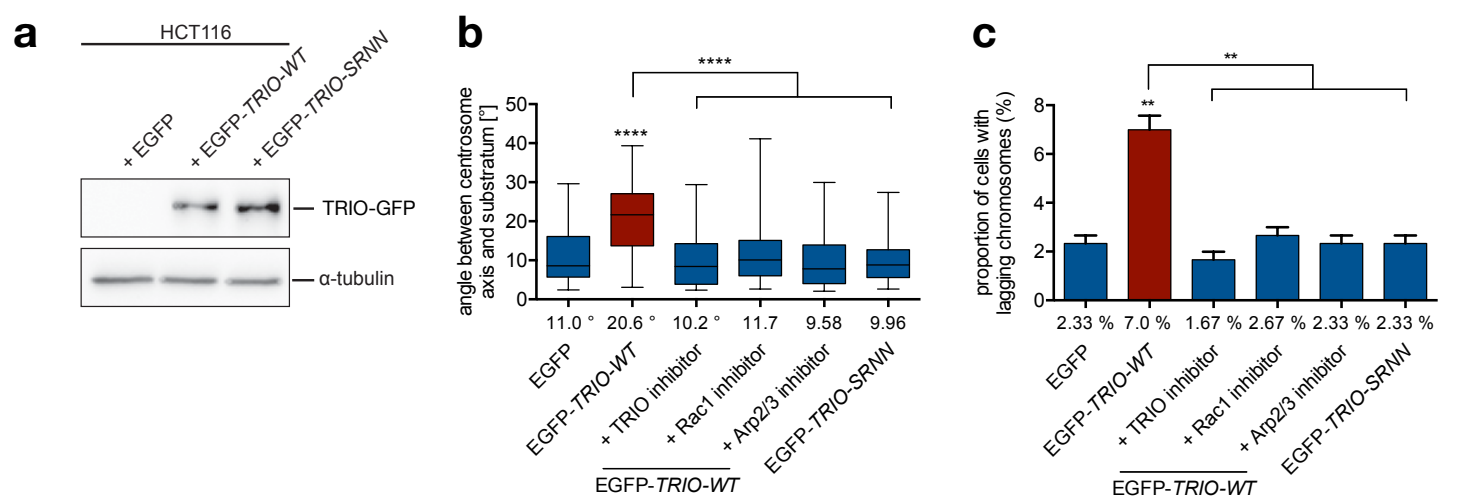

Fig. 3.24 TRIO-WT overexpression induces spindle axis misalignment during prometaphase in chromosomally stable HCT116 cells, which is dependent on EB1 and acts upstream of the Rac1Arp2/3 pathway.

(a) Overexpression of TRIO-WT and TRIO-SRNN in HCT116 cells was verified by western blot analysis. atubulin was used as a loading control. (b) Spindle axis alignment during prometaphase in chromosomally stable HCT116 cells after overexpression of TRIO-WT or an EB1 binding deficient mutant TRIO-SRNN. $48 \mathrm{~h}$ prior fixation, cells were transfected with TRIO-WT or TRIO-SRNN whereby TRIO-WT transfected cells were additionally treated with $15 \mu \mathrm{M}$ ITX3 (TRIO inhibitor), $40 \mu \mathrm{M}$ NSC23766 (Rac1 inhibitor) or $20 \mu \mathrm{M}$ CK666 (Arp2/3 inhibitor) for $16 \mathrm{~h}$. For immunofluorescence microscopy, cells were grown on fibronectin coated coverslips and fixed and stained with antibodies detecting a-tubulin and y-tubulin. The box and whisker diagram show the median (mean values were added), the 25th to 75 th percentile and the minimum and maximum values ( $n=3,60$ cells analyzed in total, $t$-test). (c) Quantification of lagging chromosomes in anaphase in TRIO-WT or TRIO-SRNN overexpressing HCT116 cells. For an accumulation of anaphases, transfected cells, as described in (b), were synchronized at G1/S-phase by a double thymidine block and inhibitors were added $8 \mathrm{~h}$ prior to fixation. After releasing the cells into the cell cycle in the presence of 
inhibitors (concentration as described in b) for $8.5 \mathrm{~h}$, cells were fixed and stained for immunofluorescence microscopy. Bar graphs show the proportion of cells showing a CENP-C positive chromatid lagging behind in anaphase $(n=3$, total amount of 300 analyzed anaphases, mean values $\pm S E M, t$-test). Indicated $p$-values refer to corresponding control cells. ${ }^{* *}: p \leq 0.01{ }^{* * * *}: p \leq 0.0001$

These results indicate that TRIO acts downstream of microtubule plus-end assembly rates but upstream of the Rac1-Arp2/3 pathway. Moreover, it was shown that TRIO-SRNN did not induce spindle axis misalignment in HCT116 cells in contrast to TRIO-WT, indicating that EB1 binding is required for its ability to activate Rac1 downstream of microtubule dynamics, which is in line with previous findings (Berger, 2016).

Taken together, the present findings indicate that a microtubule triggered TRIO-Rac1-Arp2/3 pathway deregulates the actin cytoskeleton thereby causing reduced cortical tension, which promotes spindle axis misalignment during prometaphase and subsequently chromosome missegregation in CIN cells. 


\section{Discussion}

Recently our group revealed that increased microtubule plus-end assembly rates during mitosis are characteristic for CIN cells (Ertych et al., 2014). Moreover, this work demonstrated that due to enhanced microtubule plus-end assembly rates the mitotic spindle exhibits transient geometry defects, which facilitate the generation of lagging chromosomes (Berger, 2016; Ertych et al., 2014). Recently it was revealed that a hyperactive TRIO-Rac1-Arp2/3 pathway based on enhanced microtubule plus-end assembly rates during mitosis led to spindle axis misalignment in CIN cells (Berger, 2016). However, it remains unknown how this pathway affects spindle axis alignment, and thus contributes to $\mathrm{CIN}$ and aneuploidy.

The present study is the first that systematically analyzed spindle axis alignment in a panel of colorectal cancer cells and revealed a clear correlation between enhanced microtubule plus-end assembly rates during mitosis and spindle axis misalignment in prometaphase, which resulted in the subsequent generation of lagging chromosomes in anaphase. Intriguingly, spindle axis misalignment in prometaphase was only observed in chromosomally unstable cells with enhanced microtubule plus-end assembly rates whereas MIN/MSI cells exhibited proper spindle axis alignment.

In line with previous findings from our lab (Berger, 2016), the present work verified that spindle axis misalignment in CIN cells was induced by an unscheduled TRIO-Rac1-Arp2/3 pathway downstream of enhanced microtubule plus-end assembly rates in mitosis. In detail, the present results indicate that RhoA via DIAPH1 regulates proper actin cortex architecture and cortical tension in MIN/MSI and CIN cells whereas in CIN cells an additional TRIO-Rac1-Arp2/3 pathway, based on enhanced microtubule plus-end assembly rates, results in enhanced branched actin polymerization leading to reduced cortical tension. This in turn, causes spindle axis misalignment thereby facilitating erroneous microtubule-kinetochore attachments leading to the generation of lagging chromosomes. Thus, a deregulated TRIO-Rac1-Arp2/3 actin pathway triggered by enhanced microtubule plus-end assembly rates reduces cortex tension and induces spindle axis misalignment thereby facilitating chromosome mis-segregation in human colorectal cancer cells and promoting the development of CIN and aneuploidy (Fig. 4.1).

So far, spindle orientation was primarily analyzed in metaphase cells (Kiyomitsu \& Cheeseman, 2012; Théry et al., 2007; Théry et al., 2005). Based on the observation that CIN cells exhibited abnormal spindle structures during prometaphase, which was associated with spindle axis misalignment, cells in prometaphase were analyzed. The phenotype in prometaphase was defined 
as spindle axis (mis)alignment rather than spindle orientation to distinguish between a typically analyzed metaphase cell and a transient defect, which was only observed in prometaphase cells but not in metaphase.

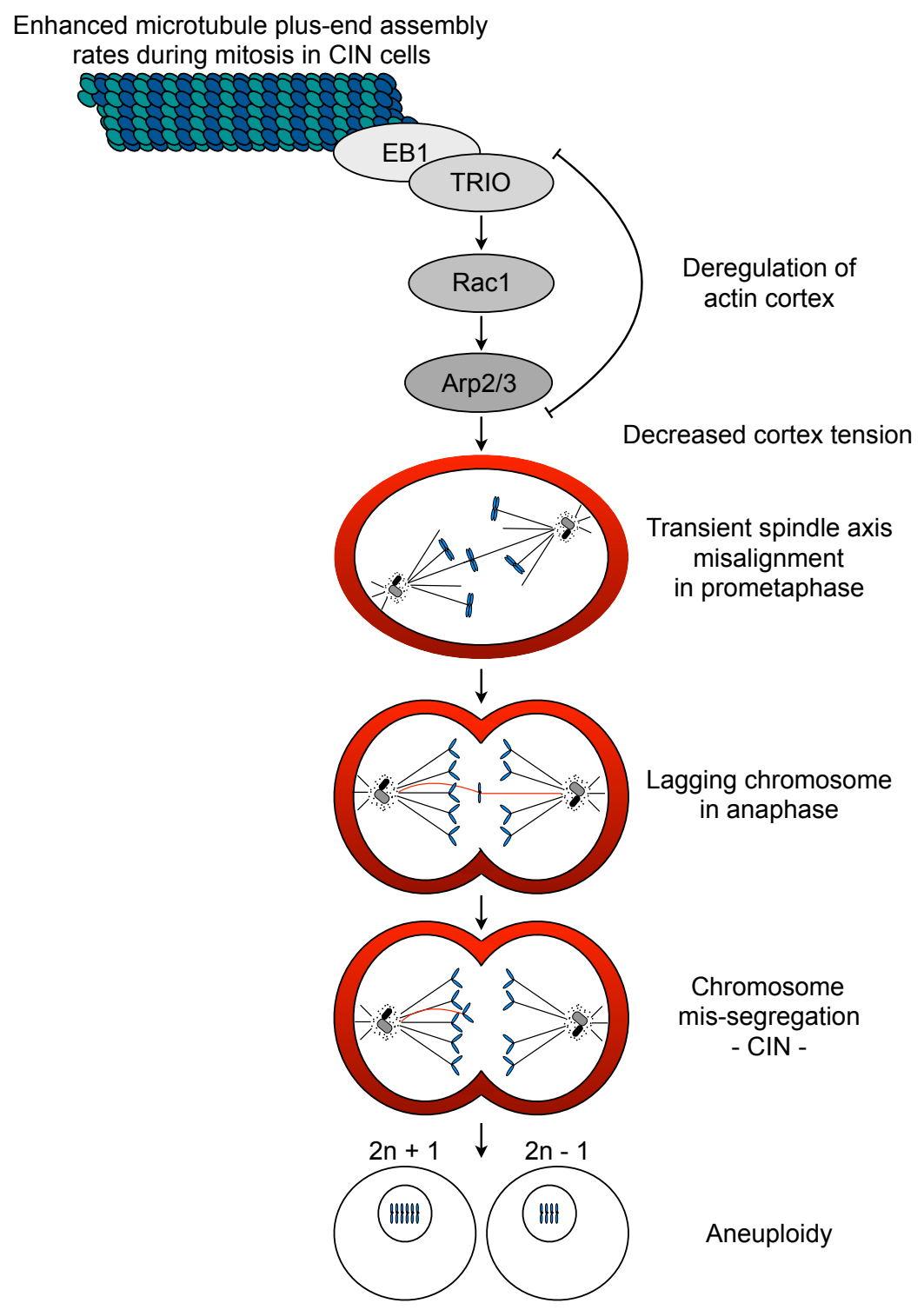

Fig. 4.1 Model illustrating the role of an unscheduled TRIO-Rac1-Arp2/3 pathway for spindle axis misalignment and the development of CIN.

Based on enhanced microtubule plus-end assembly rates an unscheduled TRIO-Rac1-Arp2/3 pathway leads to branched actin polymerization at the cortex. As a result of enhanced actin polymerization cortical tension is reduced, which promotes spindle axis misalignment in prometaphase thereby facilitating erroneous kinetochore-microtubule attachments resulting in the generation of lagging chromosomes. This in turn is the basis for chromosome mis-segregation and finally results in $\mathrm{CIN}$ and aneuploidy.

The classical pathway for spindle orientation involves the $\mathrm{Ga}_{\mathrm{i}}-\mathrm{LGN}-\mathrm{NuMA}$-dynein complex, which anchors astral microtubules at the cell cortex and pulls centrosomes towards the cell periphery to 
position the spindle (di Pietro et al., 2016). In accordance with results from several groups (Kotak, Busso, \& Gönczy, 2012; Peyre et al., 2011; Seldin, Muroyama, \& Lechler, 2016), interference with the tethering machinery for spindle orientation in chromosomally stable cells caused spindle axis misalignment in prometaphase and an increased number of cells with lagging chromosomes. Strikingly, long term spindle axis misalignment induced by pertussis toxin led to a high variability in chromosome number (unpublished data from our group). These results indicate that spindle axis misalignment per se promotes the development of CIN. Regarding tumorigenesis, spindle orientation is often analyzed in terms of controlling asymmetric or symmetric division, which leads to differentiative or proliferative cell division, respectively (Noatynska et al., 2012). Intriguingly, loss of asymmetric cell division was observed in precancerous tissue (Quyn et al., 2010) and impaired differentiative cell division was associated with tumor growth in $D$. melanogaster (Caussinus \& Gonzalez, 2005). These results emphasize the role for spindle orientation in tumorigenesis. Remarkably, spindle axis misalignment in prometaphase was not associated with CIN so far.

\section{The role of actin cortex architecture for cortical tension and spindle axis alignment}

Rac1 and Arp2/3 are known for their function during cell migration. Here, Rac1 activates the Arp2/3 complex and leads to polymerization of branched actin filaments at the leading edge resulting in structures like filopodia and lamellipodia (Blanchoin et al., 2014). For Rac1 activation several GEFs are known, among them Tiam1 and TRIO (Marei \& Malliri, 2017; Van Rijssel \& Van Buul, 2012). Interestingly, TRIO was shown to bind to microtubules in a complex with NAV1 in an EB1 dependent manner thereby enhancing Rac1 activity (Van Haren et al., 2014). Taken together, in interphase, TRIO-Rac1-Arp2/3 signaling has an established role whereas an emerging role in mitosis for spindle axis alignment was first identified by previous work from our group (Berger, 2016). Interestingly, several years ago it was shown that Arp $2 / 3$ dependent subcortical actin clouds, that polymerized based on the position of retraction fibers, helped to orient the mitotic spindle (Fink et al., 2011; Mitsushima et al., 2010). The occurrence of Arp2/3 dependent actin clouds was verified by experiments from our lab. Moreover, both, restoring proper microtubule plus-end assembly rates in CIN cells and inhibition of TRIO, Rac1 and Arp2/3, led to reduced intensity of actin clouds (unpublished date from our group) and restored proper spindle axis alignment. However, besides actin clouds, the intensity of the actin cortex was also affected (unpublished data from our group). Supporting a role for the actin cytoskeleton, work from Toyoshima et al. demonstrated that an intact actin cytoskeleton is required for proper spindle orientation in general (Toyoshima \& Nishida, 2007). In line with these findings, deregulation of 
actin polymerization caused spindle axis misalignment in MIN/MSI cells. Especially organization of the actin cortex along with myosin II was shown to be important for the generation of cortical tension (Ramanathan et al., 2015). This work suggests that actin functions as a scaffold for myosin II, which finally results in the generation of mitotic cortex tension. Moreover, RhoA instead of Arp2/3 mediated actin polymerization was shown to be required for hosting myosin II at the cell cortex (Ramanathan et al., 2015). Supporting this, Chugh et al. revealed that deregulation of the actin cortex architecture resulted in reduced cortex tension in mitotic cells. These findings indicate that cortex tension based on actin architecture rather than actin itself is important for accurate spindle axis alignment. Indeed, it was already shown that cortex tension is required for proper spindle orientation (Luxenburg et al., 2011; Rosenblatt et al., 2004). In line with these findings, CIN cells exhibiting spindle axis misalignment revealed reduced cortical tension in comparison to chromosomally stable cells. Moreover, lowering cortex tension by inhibition of myosin II resulted in inaccurate spindle axis alignment in chromosomally stable cells. Supporting the role of actin structure for cortex tension as proposed by Chugh et al., deregulation of actin dynamics by using Jasplakinolide, which induced spindle axis misalignment in MIN/MSI cells, caused reduced cortical tension in chromosomally stable cells and altered actin cortex architecture (unpublished data from our group). In contrast to chromosomally stable cells, spindle axis misalignment in CIN cells did not change after increased actin polymerization or hyperactivation of RhoA. In line with this, AFM experiments revealed that SW620 cells still exhibited reduced cortical tension after the addition of Jasplakinolide (unpublished data). However, inhibition of RhoA, myosin II or LatA treatment induced spindle axis misalignment in HCT116 cells and restored proper spindle axis alignment in CIN cells, even though AFM experiments revealed no increase in cortical tension in SW620 cells (unpublished data from our group, summary in Fig. 4.2).

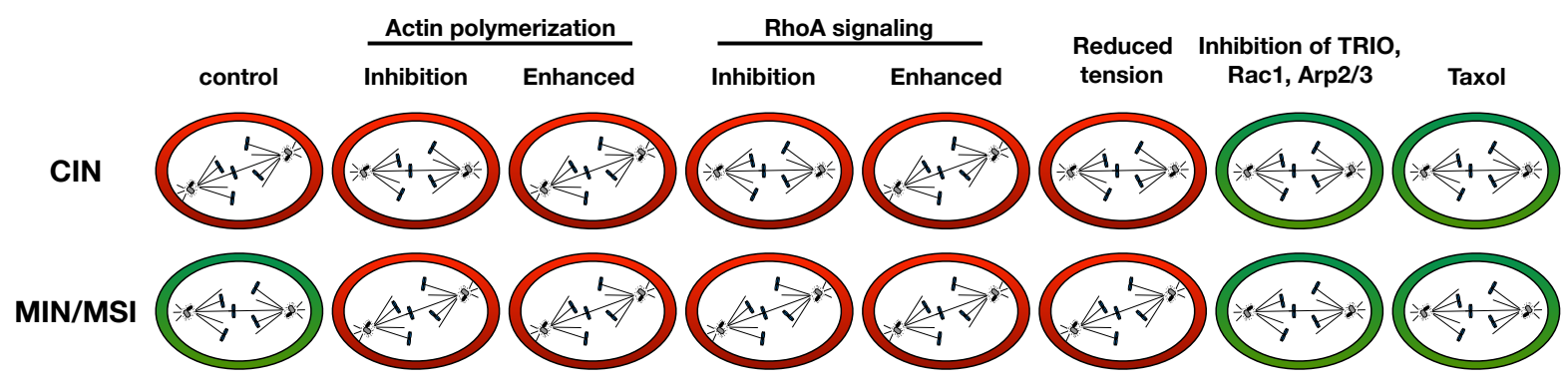

Fig. 4.2 Summary of treatments influencing cortical tension and spindle orientation.

Data from our group showed that after LatA treatment the mitotic cortex is harmed, which might result in unsuccessful cell rounding and smaller space for the mitotic spindle to position itself. In line with this, results from Lázaro-Diéguez and colleagues revealed that spindles missing astral 
microtubules still oriented in a non-random manner. In these experiments impaired cell rounding functioned as a mechanical constraint that blocked random spindle positioning (Lázaro-Diéguez, Ispolatov, \& Müsch, 2015). Thus, it might be possible that inhibition of actin polymerization or inhibition of myosin II in CIN cells seems to restore proper spindle axis alignment, but in fact, the spindle is not able to adopt another alignment due to mechanical constraints. However, it was also shown that confinement of cellular height resulted in multipolar spindles (Lancaster et al., 2013), which was shown to promote the generation of lagging chromosomes (Ganem et al., 2009). Thus, it is not clear how inhibition of actin polymerization and myosin II restored proper spindle axis alignment in CIN cells.

Intriguingly, interference with actin polymerization by treatment with drugs or deregulation of RhoA influenced spindle axis alignment in MIN and CIN cells, whereas inhibition of the TRIO-Rac1Arp2/3 pathway only affected spindle axis alignment in CIN cells (Fig. 4.2). Thus, it is likely that a RhoA-formin pathway is active in both, chromosomally stable and unstable cells to regulate unbranched actin polymerization, which promotes the generation of proper cortex tension during mitosis. Additionally, in CIN cells an unscheduled TRIO-Rac1-Arp2/3 pathway leads to enhanced branched actin polymerization, which results in reduced cortex tension thereby facilitating spindle axis misalignment in CIN cells (Fig. 4.3).
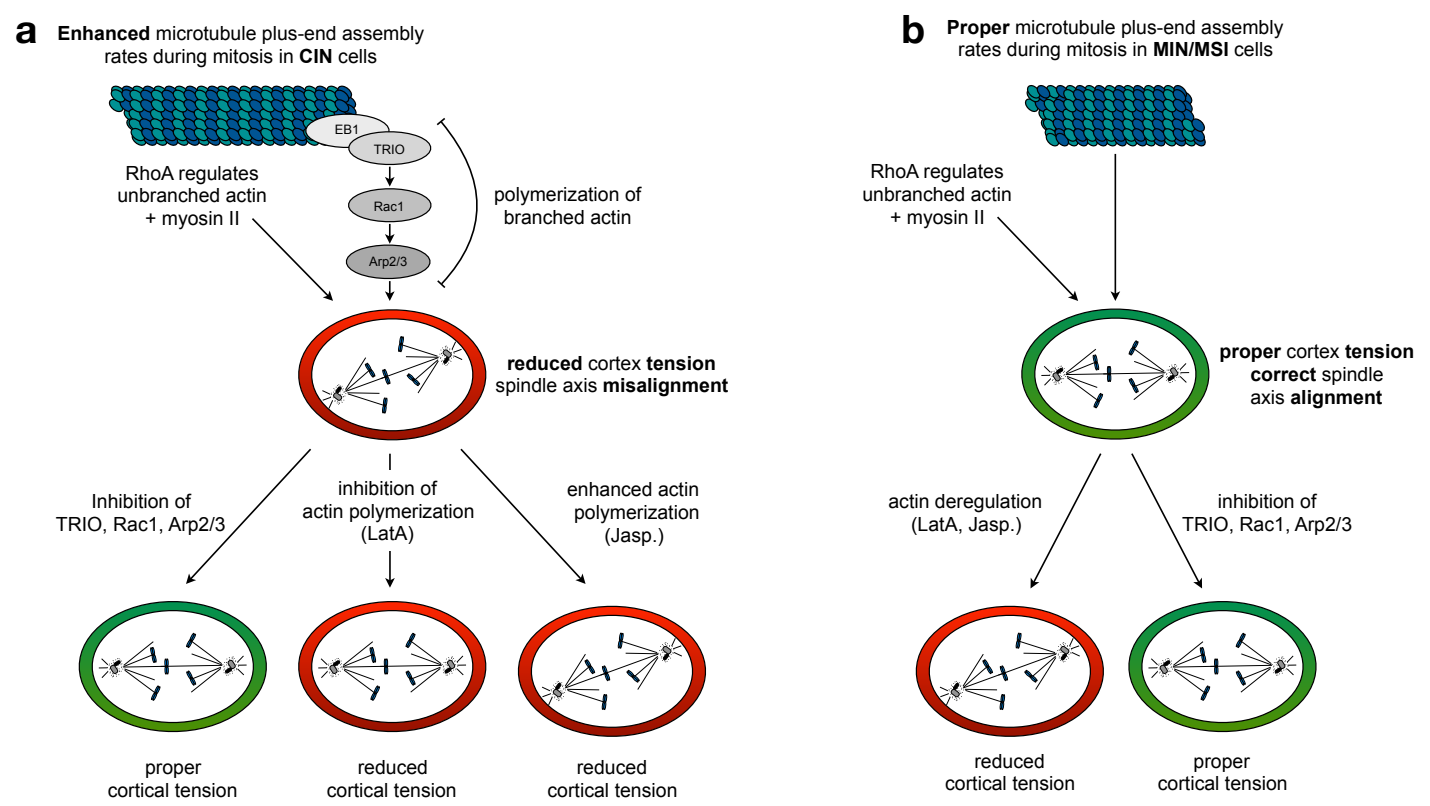

Fig. 4.3 Outcome of interfering with actin dynamics on spindle axis alignment and cortical tension. It is assumed that RhoA is active in both, CIN and MIN/MSI cells. (a) In CIN cells, increased branched actin polymerization due to a microtubule triggered TRIO-Rac1-Apr2/3 pathway caused reduced cortical tension, which resulted in spindle axis misalignment. Inhibition of TRIO, Rac1 or Arp2/3 restored cortical tension and proper spindle axis alignment. Inhibition of actin polymerization by Latrunculin A (LatA) resulted in proper spindle axis alignment even though cortical tension was not increased whereas additional actin 
polymerization by Jasplakinolide (Jasp.) did not change cortical tension or spindle axis misalignment in CIN cells. (b) The present results indicate that in MIN/MSI only RhoA is active and mediates proper actin dynamics, cortical tension and spindle axis alignment. Actin deregulation, enhanced or reduced actin polymerization, led to spindle axis misalignment and reduced cortical tension whereas inhibition of TRIO, Rac1 and Arp2/3 had no impact on spindle axis alignment or cortex tension.

Emphasizing the role of TRIO, Rac1 and Arp2/3 for reduced cortical tension in CIN cells, AFM experiments revealed that inhibition of these proteins increased cortex tension in SW620 cells to a level, which was observed in HCT116 cells. Moreover, cortical tension in HCT116 cells was not affected by inhibition of Rac1 (unpublished data). Intriguingly, unpublished data from our group revealed that overexpression of TRIO caused increased Rac1 activity, which was dependent on its ability to bind to EB1. Moreover, single cell clones overexpressing TR/O wildtype exhibited a high chromosome number variability whereas a TRIO mutant, which is not able to bind to EB1 showed a stable karyotype (unpublished data from our group). In line with this, our group revealed that TRIO was often observed in colorectal cancer with a CIN phenotype while TRIO expression correlated with a high variability in chromosome number in cells of the $\mathrm{NCl}-60$ panel (unpublished data from our group).

Taken together, these findings indicate that formin mediated unbranched actin polymerization is responsible for proper cortical tension whereas in CIN cells unscheduled TRIO-Rac1-Arp2/3 signaling results in branched actin polymerization, which seems to impair proper cortex tension. Supporting this, work form Reymann et al. demonstrated that branched actin structures exhibited reduced contractility in comparison to unbranched, antiparallel organized actin bundles (Reymann et al., 2012). Moreover, it was shown that besides actin architecture the extend of connected actin filaments plays an important role for actomyosin contractility (Ennomani et al., 2016). This work revealed that an actin network composed of moderate connected, antiparallel organized actin bundles was most suitable for actomyosin based contractility. Remarkably, an increase and decrease of connectors such as Arp2/3 or $\alpha$-actinin led to reduced contractility whereas lowering connectivity in highly connected actin structures resulted in higher contractility (Ennomani et al., 2016). The authors proposed that a high amount of connected actin filaments prevents contraction by myosin II. These findings further strengthen the hypothesis that based on RhoA, unbranched actin filaments promote proper cortex tension whereas in CIN cells Arp2/3 activity results in highly connected actin structures, which reduce cortical tension (Fig. 4.4).

\section{How does reduced cortical tension cause spindle axis misalignment?}

However, it is still unclear how reduced cortical tension results in spindle axis misalignment. One possibility might be that proper cortex tension is required for correct localization of the LGN-NuMA 
complex (Luxenburg et al., 2011). To verify this, it is required to analyze the localization of the LGN-NuMA complex in either fixed or living cells. Furthermore, it would be interesting to compare localization of LGN-NuMA in CIN and MIN cells and if influencing cortex tension or actin structures has an impact on localization of the LGN-NuMA complex.
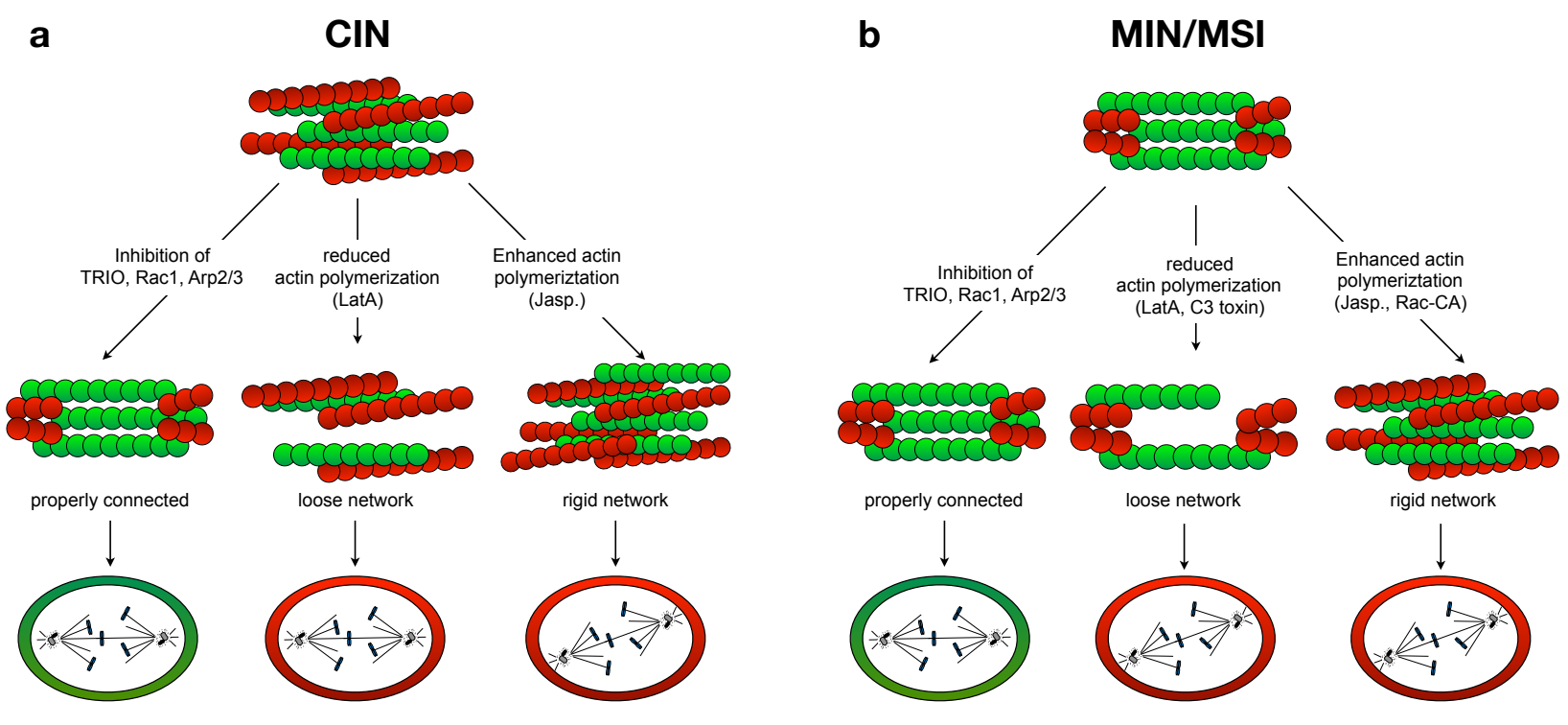

Fig. 4.4 Model of how actin cortex architecture influences cortical tension.

(a) In CIN cells a rigid actin network leads to decreased cortical tension. Only inhibition of TRIO, Rac1 and Arp2/3 restores proper cortical tension and spindle axis alignment. Both, inhibition and enhanced actin polymerization results in an actin network that is not suitable for hosting myosin II. (b) MIN/MSI cells exhibit an optimal actin network for hosting myosin II. Deregulation of actin dynamics results in a too loose or rigid network that is not able to host myosin II at the cortex. As a result, cortical tension is reduced and leads to spindle axis misalignment.

Another way of cortical tension to influence spindle axis alignment was revealed by Rosenblatt and colleagues who showed that a cortical flow dependent on myosin II separated and positioned centrosomes via cortical anchored astral microtubules (Rosenblatt et al., 2004). Cortical flow describes a process that moves f-actin, myosin II and other cortex associated proteins within the cell cortex (Benink et al., 2000; Bray \& White, 1988). This movement was shown to be dependent on actomyosin contraction whereby its oriented towards sites of high contraction and away from centrosomes (Benink et al., 2000). In line with this, work from De Simone and colleagues suggests that centrosomes are able to induce a cortical flow moving them from poles towards the cell equator thereby facilitating centrosome separation (De Simone et al., 2016). Interestingly, microtubule polymerization inhibits cortical flow while depolymerization promotes this process (Mandato, Benink, \& Bement, 2000). Microtubule depolymerization was shown to release GEF$\mathrm{H} 1$ which resulted in activation of RhoA (Krendel, Zenke, \& Bokoch, 2002) thereby promoting actomyosin contraction via activation of ROCK (Ren, Kiosses, \& Schwartz, 1999). Contrary, 
microtubule polymerization resulted in Rac1 activation (Waterman-Storer et al., 1999) and inhibited contractility via PAK and leads to actin polymerization (Mandato et al., 2000). Thus, it is possible that due to enhanced microtubule plus-end assembly rates, a higher amount of GEF-H1 is bound to microtubules thereby preventing RhoA activation, which reduces cortical tension and might also impair cortical flow, which in turn influences centrosome separation. Additionally, based an increased microtubule plus-end assembly rates, Rac1 might lead to activation of PAK thereby blocking phosphorylation of MLC. Taken together, it is possible that astral microtubules deliver TRIO to the cell cortex where it activates Rac1, which leads to branched actin polymerization via Arp2/3. This in turn, might results in reduced cortical tension. Additionally, microtubules could directly influence cortex tension by sequestering GEF-H1 thereby suppressing RhoA and MLC via PAK activation by Rac1 (Fig. 4.5).
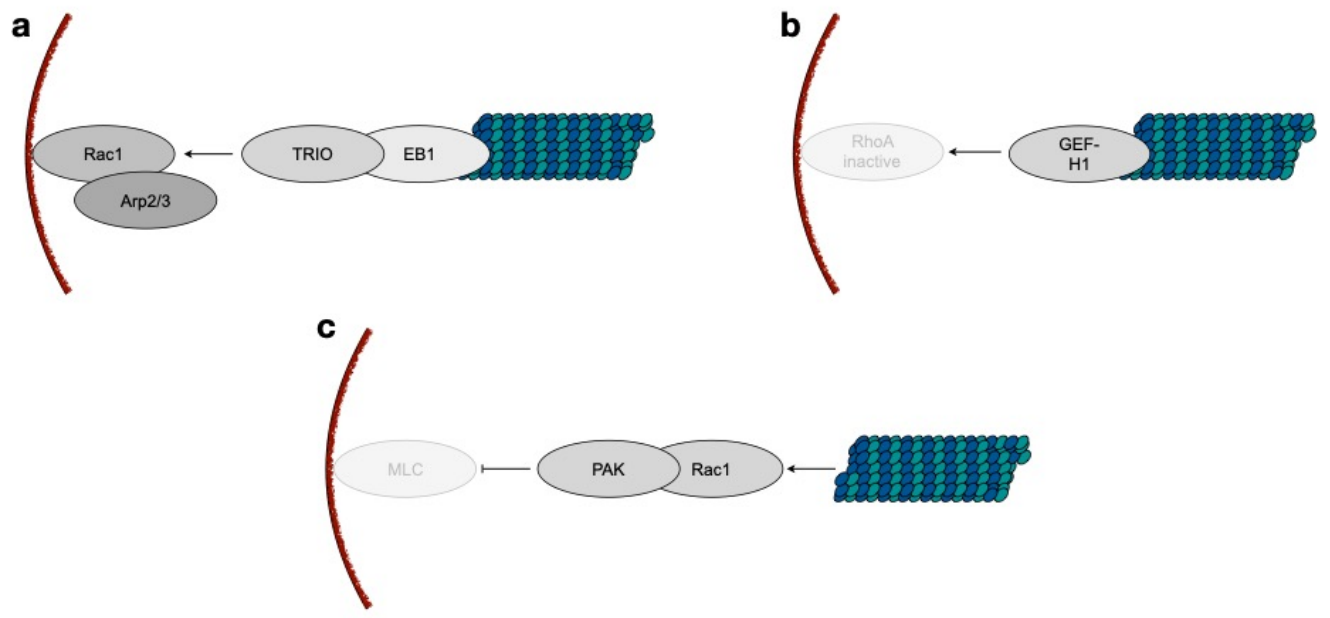

Fig. 4.5 Models to reduce cortical tension by enhanced microtubule plus-end assembly rates during mitosis.

(a) Due to enhanced microtubule plus-end assembly rates TRIO-Rac1-Arp2/3 signaling is hyperactive and leads to branched actin polymerization. (b) Binding of GEF-H1 to microtubules prevents RhoA activation. (c) Microtubule polymerization results in Rac1 mediated PAK activation, which leads to inhibition of MLC of myosin II.

\section{Rac1 at the centrosome influences centrosome dynamics}

However, Rac1 is not only localized at the cell cortex but also at the centrosome (Payapilly \& Malliri, 2018). Additionally, EB1 was reported to localize at centrosomes as well (Louie et al., 2004). Especially localization of Rac1 at the centrosome was shown to be cell cycle dependent since Rac1 was found at centrosomes only during prophase and prometaphase (Woodcock et al., 2010). Interestingly, it was revealed that Rac1 opposes Eg5 mediated centrosome separation via PAK1/2 activation at centrosomes in a Tiam1 dependent manner (Whalley et al., 2015b; Woodcock et al., 2010). The previous function of PAK at centrosomes involved activation of 
AuroraA kinase to facilitate centrosome maturation (Fukasawa, 2007) whereby inhibition of PAK resulted in delayed centrosome maturation (Zhao et al., 2005). However, Woodcock and colleagues proposed that reduced Rac1 and PAK activity based on Tiam1 depletion results in faster centrosome separation leading to prolonged prometaphase and chromosome congression defects (Woodcock et al., 2010). This raised the question if CIN cells exhibit slower centrosome separation due to enhanced Rac1 activity. Surprisingly, initial experiments during this thesis showed that SW620 cells executed early centrosome separation faster than HCT116 cells. Still, chromosome congression and onset of anaphase was delayed in CIN cells. Intriguingly, restoring proper microtubule dynamics in SW620 cells reduced the velocity of centrosome separation to a level, which was observed in HCT116 cells. Interestingly, accelerated centrosome separation is associated with spindle geometry defects and lagging chromosomes (Nam et al., 2015). Moreover, I performed initial experiments showing that SW620 cells were less sensitive towards Eg5 inhibition, which was in contrast to Woodcock et al. who proposed that reduced Rac1 activity leads to decreased sensitivity towards Eg5 inhibition (Woodcock et al., 2010). Those first results indicate that increased centrosome separation due to enhanced microtubule plus-end assembly rates might lead to erroneous kinetochore-microtubule attachments. This in turn results in error correction, which delays anaphase onset until kinetochore-microtubule attachments are corrected. Thus, the observed effects of accelerated centrosome separation are in line with results from Woodcock and colleagues (Woodcock et al., 2010) even though enhanced, instead of reduced Rac1 activity might be the cause for this phenotype in CIN cells. Supporting a role for Tiam1, initial experiments during this thesis showed that depletion of Tiam1 resulted in restoration of proper spindle axis alignment in CIN cells whereas overexpression of Tiam1 was efficient to induce spindle axis misalignment in HCT116 cells. Therefore, it is possible that Rac1 functions also at the centrosome regulating centrosome separation in CIN cells thereby influencing spindle orientation in CIN cells. Moreover, it cannot be ruled out that cortical and centrosomal Rac1 cooperate to position and orient the mitotic spindle.

\section{How does spindle axis misalignment promote the generation of lagging chromosomes?}

It was already revealed that CIN cells with enhanced microtubule dynamics exhibit hyper-stable kinetochore-microtubule attachments, which facilitate the generation of lagging chromosomes (Bakhoum et al., 2009; Ertych et al., 2014). Furthermore, multipolar spindles caused by supernumerary centrosomes were shown to promote merotelic attachments by a transient change of spindle geometry thereby facilitating the generation of lagging chromosomes (Ganem et al., 2009). Additionally, work from Nam and colleagues demonstrated that accelerated centrosome 
separation resulted in spindle geometry defects, merotelic attachments and lagging chromosomes (Nam \& van Deursen, 2014). The authors propose that accelerated centrosomes lead to lateral attachment of microtubules to kinetochores, which are not mature in early prometaphase thereby facilitating merotelic attachments (Nam et al., 2015). Likewise, delayed centrosome separation was also shown to result in spindle geometry defects and facilitate the generation of lagging chromosomes due to merotelic attachments (Silkworth \& Cimini, 2012). This work demonstrates that spindle geometry defects promoted merotelic attachments immediately or via transient syntelic attachments, which can result in merotely (Silkworth \& Cimini, 2012). During this thesis, initial experiments revealed that CIN cells exhibited increased centrosome separation, which indicates that merotely results from attachment of microtubules to immature kinetochores. Moreover, spindle geometry defects described above might include transient spindle axis misalignment in prometaphase, and thus leading to merotelic attachments and lagging chromosomes, which finally results in the development of CIN.

\section{Rac1 and TRIO in cancer}

Rac1 can be activated by several GEFs including Tiam1 and TRIO (Marei \& Malliri, 2017; Van Rijssel \& Van Buul, 2012). Those were found to be overexpressed in tumor tissues whereby especially TRIO overexpression correlates with invasiveness and poor patients outcome (J. Lane, Martin, Mansel, \& Jiang, 2008; Salhia et al., 2008; M. Zheng et al., 2004). Furthermore, TRIO was identified to drive migration in cells of the NCl-60 cell panel (Kohn et al., 2012) and is part of the HET70 signature, which involves genes highly associated with aneuploidy (Sheltzer, 2013). Likewise, overexpression of Rac1 and its downstream effector Arp2/3 is observed in several cancers and promotes tumorigenesis (Wertheimer et al., 2012; H. Zheng et al., 2008). Thus, it would be of great interest to analyze if tumors overexpressing TRIO, Rac1 or Arp2/3 reveal spindle axis misalignment in prometaphase, increased centrosome separation and reduced cortex tension. Intriguingly, initial studies showed that cancer cells exhibit decreased contractility and especially metastatic cells reveal reduced stiffness (Cross et al., 2007; Remmerbach et al., 2009). In line with these findings, previous results from our group showed that enhanced microtubule plus-end assembly rates in interphase led to increased cell migration and invasion (Berger, 2016). Moreover, Bakhoum and colleagues reported that CIN promotes metastasis. This work revealed that CIN facilitated metastasis by generating micronuclei, when disrupted, resulted in cytosolic DNA thereby promoting metastasis via constant activation of the immune system (Bakhoum et al., 2018). Since Rho GTPases and especially Rac1 are promising targets for anti-cancer therapy, there are already several inhibitors developed even though most of them are not suitable for 
clinical approaches (Mardilovich, Olson, \& Baugh, 2012). The Rac1 inhibitor NSC23766 was shown to prevent Rac1 activity by blocking activation via Tiam1 and TRIO (Gao et al., 2004) and resulted in apoptosis and G1 cell cycle arrest (Yoshida et al., 2010). However, NSC23766 revealed a high $\mathrm{IC}_{50}$ and was proven to be inefficient for clinical application (Bid et al., 2013). Other Rac1 inhibitors were developed, among them EHop-016, which revealed a lower $I_{50}$ and was shown to be more efficient. However, EHop-016 inhibits the interaction between Rac1 and another GEF called Vav2 instead of TRIO (Montalvo-Ortiz et al., 2012). The established TRIO inhibitor ITX3 was also not suitable for clinical use due to its high IC $_{50}$ (Bouquier et al., 2009; Del Mar Maldonado \& Dharmawardhane, 2018). Thus, it is of great interest to develop small molecule inhibitors targeting the TRIO-Rac1-Arp2/3 pathway since suppression might be able to inhibit both, enhanced migration and invasion as well as decreased cortical tension and thus preventing the development of CIN. 


\section{References}

Akhmanova, A., \& Steinmetz, M. O. (2008). Tracking the ends: A dynamic protein network controls the fate of microtubule tips. Nature Reviews Molecular Cell Biology, 9(4), 309-322. https://doi.org/10.1038/nrm2369

Akhmanova, A., \& Steinmetz, M. O. (2015). Control of microtubule organization and dynamics: Two ends in the limelight. Nature Reviews Molecular Cell Biology, 16, 711-726. https://doi.org/10.1038/nrm4084

Anderhub, S. J., Krämer, A., \& Maier, B. (2012). Centrosome amplification in tumorigenesis. Cancer Letters, 322(1), 8-17. https://doi.org/10.1016/j.canlet.2012.02.006

Andrianantoandro, E., \& Pollard, T. D. (2006). Mechanism of Actin Filament Turnover by Severing and Nucleation at Different Concentrations of ADF/Cofilin. Molecular Cell, 24(1), 13-23. https://doi.org/10.1016/j.molcel.2006.08.006

Antonin, W., \& Neumann, H. (2016). Chromosome condensation and decondensation during mitosis. Current Opinion in Cell Biology, 40, 15-22. https://doi.org/10.1016/j.ceb.2016.01.013

Arakawa, Y., Bito, H., Furuyashiki, T., Tsuji, T., Takemoto-Kimura, S., Kimura, K., ... Narumiya, S. (2003). Control of axon elongation via an SDF-1 $\alpha /$ Rho/mDia pathway in cultured cerebellar granule neurons. Journal of Cell Biology, 161(2), 381-391. https://doi.org/10.1083/jcb.200210149

Bakhoum, S. F., \& Cantley, L. C. (2018). The Multifaceted Role of Chromosomal Instability in Cancer and Its Microenvironment. Cell, 174(6), 1347-1360.

https://doi.org/10.1016/j.cell.2018.08.027

Bakhoum, S. F., Danilova, O. V., Kaur, P., Levy, N. B., \& Compton, D. A. (2011). Chromosomal instability substantiates poor prognosis in patients with diffuse large B-cell lymphoma. Clinical Cancer Research, 17(24), 7704-7711. https://doi.org/10.1158/1078-0432.CCR-112049

Bakhoum, S. F., Ngo, B., Laughney, A. M., Cavallo, J. A., Murphy, C. J., Ly, P., .. Cantley, L. C. 
(2018). Chromosomal instability drives metastasis through a cytosolic DNA response. Nature, 553(7689), 467-472. https://doi.org/10.1038/nature25432

Bakhoum, S. F., Silkworth, W. T., Nardi, I. K., Nicholson, J. M., Compton, D. A., \& Cimini, D. (2014). The mitotic origin of chromosomal instability. Current Biology, 24(4), 1-7. https://doi.org/10.1038/jid.2014.371

Bakhoum, S. F., Thompson, S. L., Manning, A. L., \& Compton, D. A. (2009). Genome stability is ensured by temporal control of kinetochore-microtubule dynamics. Nature Cell Biology, 11(1), 27-35. https://doi.org/10.1038/ncb1809

Bao, W., Thullberg, M., Zhang, H., Onischenko, A., \& Stromblad, S. (2002). Cell Attachment to the Extracellular Matrix Induces Proteasomal Degradation of p21CIP1 via Cdc42/Rac1 Signaling. Molecular and Cellular Biology, 22(13), 4587-4597. https://doi.org/10.1128/mcb.22.13.4587-4597.2002

Barber, T. D., McManus, K., Yuen, K. W. Y., Reis, M., Parmigiani, G., Shen, D., ... Hieter, P. (2008). Chromatid cohesion defects may underlie chromosome instability in human colorectal cancers. Proceedings of the National Academy of Sciences. https://doi.org/10.1073/pnas.0712384105

Barisic, M., Aguiar, P., Geley, S., \& Maiato, H. (2014). Kinetochore motors drive congression of peripheral polar chromosomes by overcoming random arm-ejection forces. Nature Cell Biology, 16(12), 1249-1256. https://doi.org/10.1038/ncb3060

Bartolini, F., Moseley, J. B., Schmoranzer, J., Cassimeris, L., Goode, B. L., \& Gundersen, G. G. (2008). The formin mDia2 stabilizes microtubules independently of its actin nucleation activity. Journal of Cell Biology, 181(3), 523-536. https://doi.org/10.1083/jcb.200709029

Belletti, B., \& Baldassarre, G. (2011). Stathmin : a protein with many tasks . New biomarker and potential target in cancer. Expert Opinion on Therapeutic Targets, 15(11), 1249-1266. https://doi.org/10.1517/14728222.2011.620951

Bement, W. M., Benink, H. A., \& Von Dassow, G. (2005). A microtubule-dependent zone of active RhoA during cleavage plane specification. Journal of Cell Biology, 170(1), 91-101. https://doi.org/10.1083/jcb.200501131

Benink, H. A., Mandato, C. A., \& Bement, W. M. (2000). Analysis of cortical flow models in vivo. 
Molecular Biology of the Cell, 11(8), 2553-2563. https://doi.org/10.1091/mbc.11.8.2553

Berger, K. (2016). A Role For Microtubule Dynamics For The Induction Of Chromosomal Instability And Cell Migration And Invasion In Human Cancer Cells.

Bertoli, C., Skotheim, J. M., \& De Bruin, R. A. M. (2013). Control of cell cycle transcription during G1 and S phases. Nature Reviews Molecular Cell Biology, 14(8), 518-528. https://doi.org/10.1038/nrm3629

Bid, H. K., Roberts, R. D., Manchanda, P. K., \& Houghton, P. J. (2013). RAC1: An Emerging Therapeutic Option for Targeting Cancer Angiogenesis and Metastasis. Molecular Cancer Therapeutics. https://doi.org/10.1158/1535-7163.mct-13-0164

Birkbak, N. J., Eklund, A. C., Li, Q., McClelland, S. E., Endesfelder, D., Tan, P., ... Swanton, C. (2011). Paradoxical relationship between chromosomal instability and survival outcome in cancer. Cancer Research, 71(10), 3447-3452. https://doi.org/10.1158/0008-5472.CAN-103667

Blanchoin, L., Boujemaa-Paterski, R., Sykes, C., \& Plastino, J. (2014). Actin Dynamics, Architecture, and Mechanics in Cell Motility. Physiol Rev, 94(1), 235-263. https://doi.org/10.1152/physrev.00018.2013.-Tight

Block, J., Stradal, T. E. B., Hänisch, J., Geffers, R., Köstler, S. A., Urban, E., .. Faix, J. (2008). Filopodia formation induced by active mDia2/Drf3. Journal of Microscopy, 231(3), 506-517. https://doi.org/10.1111/j.1365-2818.2008.02063.x

Booher, R. N., Holman, P. S., \& Fattaey, A. (1997). Human Myt1 is a cell cycle-regulated kinase that inhibits Cdc2 but not Cdk2 activity. Journal of Biological Chemistry, 272(35), 2230022306. https://doi.org/10.1074/jbc.272.35.22300

Bos, J. L., Rehmann, H., \& Wittinghofer, A. (2007). GEFs and GAPs: Critical Elements in the Control of Small G Proteins. Cell, 129, 865-877. https://doi.org/10.1016/j.cell.2007.05.018

Bouquier, N., Vignal, E., Charrasse, S., Weill, M., Schmidt, S., Léonetti, J. P., ... Fort, P. (2009). A Cell Active Chemical GEF Inhibitor Selectively Targets the Trio/RhoG/Rac1 Signaling Pathway. Chemistry and Biology, 16(6), 657-666.

https://doi.org/10.1016/j.chembiol.2009.04.012 
Brandt, D. T., \& Grosse, R. (2007). Get to grips: Steering local actin dynamics with IQGAPs. EMBO Reports, 8(11), 1019-1023. https://doi.org/10.1038/sj.embor.7401089

Bray, D., \& White, J. G. (1988). Cortical Flow in Animal Cells. Science, 239(4842), 883-888.

Bresnick, A. R. (1999). Molecular mechanisms of nonmuscle myosin-Il regulation. Current Opinion in Cell Biology, 11(1), 26-33. https://doi.org/10.1016/S0955-0674(99)80004-0

Bretscher, A., Edwards, K., \& Fehon, R. G. (2002). ERM proteins and merlin: Integrators at the cell cortex. Nature Reviews Molecular Cell Biology, 3(8), 586-599. https://doi.org/10.1038/nrm882

Brouhard, G. J., Stear, J. H., Noetzel, T. L., Al-Bassam, J., Kinoshita, K., Harrison, S. C., ... Hyman, A. A. (2008). XMAP215 Is a Processive Microtubule Polymerase. Cell, 132(1), 79_ 88. https://doi.org/10.1016/j.cell.2007.11.043

Bubb, M. R., Senderowiczf, A. M. J., Sausvillef, E. K., Duncanf, K. L. K., \& Kern, E. D. (1994). Jasplakinolide, a Cytotoxic Natural Product, Induces Actin Polymerization and Competitively Inhibits the Binding of Phalloidin to F-actin*. THE JOURNAL OF BIOLOGICAL CHEMISTRY, 269(21), 14869-14871.

Bugyi, B., \& Carlier, M.-F. (2010). Control of Actin Filament Treadmilling in Cell Motility. Review in Advance on Annu. Rev. Biophys, 39(1), 449-470. https://doi.org/10.1146/annurevbiophys-051309-103849

Burridge, K., \& Wennerberg, K. (2004). Rho and Rac Take Center Stage. Cell, 116, 167-179. https://doi.org/10.1016/S0092-8674(04)00003-0

Cahill, D. P., Kinzler, K. W., Vogelstein, B., \& Lengauer, C. (1999). Genetic instability and darwinian selection in tumours. Trends in Biochemical Sciences, 24(12), 57-60. https://doi.org/10.1016/S0968-0004(99)01466-8

Campellone, K. G., \& Welch, M. D. (2010). A nucleator arms race: Cellular control of actin assembly. Nature Reviews Molecular Cell Biology, 11, 237-251. https://doi.org/10.1038/nrm2867

Carmena, M., Wheelock, M., Funabiki, H., \& Earnshaw, W. C. (2012). The chromosomal passenger complex (CPC): From easy rider to the godfather of mitosis. Nature Reviews 
Molecular Cell Biology, 13(12), 789-803. https://doi.org/10.1038/nrm3474

Carminati, M., Gallini, S., Pirovano, L., Alfieri, A., Bisi, S., \& Mapelli, M. (2016). Concomitant binding of Afadin to LGN and F-actin directs planar spindle orientation. Nature Structural and Molecular Biology, 23(2), 155-163. https://doi.org/10.1038/nsmb.3152

Carter, S. L., Eklund, A. C., Kohane, I. S., Harris, L. N., \& Szallasi, Z. (2006). A signature of chromosomal instability inferred from gene expression profiles predicts clinical outcome in multiple human cancers. Nature Genetics, 38(9), 1043-1048.

https://doi.org/10.1038/ng1861

Caussinus, E., \& Gonzalez, C. (2005). Induction of tumor growth by altered stem-cell asymmetric division in Drosophila melanogaster. Nature Genetics, 37(10), 1125-1129. https://doi.org/10.1038/ng1632

Charrasse, S., Mazel, M., Taviaux, S., Berta, P., Chow, T., \& Larroque, C. (1995).

Characterization of the CDNA and Pattern of Expression of a New Gene Over-Expressed in Human Hepatomas and Colonic Tumors. European Journal of Biochemistry, 234(2), 406413. https://doi.org/10.1111/j.1432-1033.1995.406_b.x

Cheeseman, I. M. (2014). The kinetochore. International Review of Cytology, 94(7), 77-105. https://doi.org/10.1101/cshperspect.a015826

Cheeseman, I. M., \& Desai, A. (2008). Molecular architecture of the kinetochore-microtubule interface. Nature Reviews Molecular Cell Biology, 9(1), 33-46. https://doi.org/10.1038/nrm2310

Chesarone, M. A., Dupage, A. G., \& Goode, B. L. (2010). Unleashing formins to remodel the actin and microtubule cytoskeletons. Nature Reviews Molecular Cell Biology, 11(1), 62-74. https://doi.org/10.1038/nrm2816

Chircop, M. (2014). Rho GTPases as regulators of mitosis and cytokinesis in mammalian cells. Small GTPases, 5(2), e19770. https://doi.org/10.4161/sgtp.29770

Chugh, P., Clark, A. G., Smith, M. B., Cassani, D. A. D., Dierkes, K., Ragab, A., ... Paluch, E. K. (2017). Actin cortex architecture regulates cell surface tension. Nature Cell Biology, 19(6), 689-697. https://doi.org/10.1038/ncb3525 
Cimini, D. (2007). Detection and correction of merotelic kinetochore orientation by Aurora B and its partners. Cell Cycle, 6(13), 1558-1564. https://doi.org/10.4161/cc.6.13.4452

Cimini, D., Howell, B., Maddox, P., Khodjakov, A., Degrassi, F., \& Salmon, E. D. (2001). Merotelic Kinetochore Orientation Is a Major Mechanism of Aneuploidy in Mitotic Mammalian Tissue Cells. The Journal of Cell Biology, 153(3), 517-528.

https://doi.org/10.1083/jcb.153.3.517

Conti, M. A., \& Adelstein, R. S. (2008). Nonmuscle myosin II moves in new directions. Journal of Cell Science, 121(1), 11-18. https://doi.org/10.1242/jcs.03496

Couwenbergs, C., Spilker, A. C., \& Gotta, M. (2004). Control of embryonic spindle positioning and Ga activity by C. elegans RIC-8. Current Biology, 14(20), 1871-1876.

https://doi.org/10.1016/j.cub.2004.09.059

Cross, S. E., Jin, Y. S., Rao, J., \& Gimzewski, J. K. (2007). Nanomechanical analysis of cells from cancer patients. Nature Nanotechnology, 2(12), 780-783. https://doi.org/10.1038/nnano.2007.388

Cytrynbaum, E. N., Scholey, J. M., \& Mogilner, A. (2003). A force balance model of early spindle pole separation in Drosophila embryos. Biophysical Journal, 84(2), 757-769. https://doi.org/10.1016/S0006-3495(03)74895-4

D’Assoro, A. B., Lingle, W. L., \& Salisbury, J. L. (2002). Centrosome amplification and the development of cancer. Oncogene, 21(40), 6146-6153.

https://doi.org/10.1038/sj.onc.1205772

Daub, H., Gevaert, K., Vandekerckhove, J., \& Hall, A. (2001). Rac / Cdc42 and p65PAK Regulate the Microtubule-destabilizing Protein Stathmin through Phosphorylation at Serine 16. THE JOURNAL OF BIOLOGICAL CHEMISTRY, 276(3), 1677-1680. https://doi.org/10.1074/jbc.C000635200

David, N. B., Martin, C. A., Segalen, M., Rosenfeld, F., Schweisguth, F., \& Bellaïche, Y. (2005). Drosophila Ric-8 regulates Gai cortical localization to promote Gai-dependent planar orientation of the mitotic spindle during asymmetric cell division. Nature Cell Biology. https://doi.org/10.1038/ncb1319

De Simone, A., Nédélec, F., \& Gönczy, P. (2016). Dynein Transmits Polarized Actomyosin 
Cortical Flows to Promote Centrosome Separation. Cell Reports, 14(9), 2250-2262. https://doi.org/10.1016/j.celrep.2016.01.077

Dehmelt, L., \& Halpain, S. (2004). Protein family review The MAP2 / Tau family of microtubuleassociated proteins. Genome Biology, 6(1), 1-10. Retrieved from http://genomebiology.com/2004/6/1/204

Del Mar Maldonado, M., \& Dharmawardhane, S. (2018). Targeting rac and Cdc42 GT pases in cancer. Cancer Research, 78(12), 3101-3111. https://doi.org/10.1158/0008-5472.CAN-180619

Derivery, E., \& Gautreau, A. (2010). Generation of branched actin networks: Assembly and regulation of the N-WASP and WAVE molecular machines. BioEssays, 32(2), 119-131. https://doi.org/10.1002/bies.200900123

di Pietro, F., Echard, A., \& Morin, X. (2016). Regulation of mitotic spindle orientation: an integrated view. EMBO Reports, 17(8), 1106-1130.

https://doi.org/10.15252/embr.201642292

Dimitrov, A., Quesnoit, M., Moutel, S., Cantaloube, I., Poüs, C., \& Perez, F. (2008). Detection of GTP-tubulin conformation in vivo reveals a role for GTP remnants in microtubule rescues. Science, 322(5906), 1353-1356. https://doi.org/10.1126/science.1165401

Dogterom, M., Kerssemakers, J. W. J., Romet-Lemonne, G., \& Janson, M. E. (2005). Force generation by dynamic microtubules. Current Opinion in Cell Biology, 17(1), 67-74. https://doi.org/10.1016/j.ceb.2004.12.011

Dogterom, M., \& Koenderink, G. H. (2019). Actin-microtubule crosstalk in cell biology. Nature Reviews Molecular Cell Biology, 20(1), 38-54. https://doi.org/10.1038/s41580-018-0067-1

Dransart, E., Olofsson, B., Cherfils, J., \& Munksgaard, B. (2005). RhoGDIs Revisited: Novel Roles in Rho Regulation. Traffic, 6, 957-966. https://doi.org/10.1111/j.16000854.2005.00335.x

Du, Q., \& Macara, I. G. (2004). Mammalian Pins is a conformational switch that links NuMA to heterotrimeric G proteins. Cell, 119(4), 503-516. https://doi.org/10.1016/j.cell.2004.10.028

Edwards, M., Zwolak, A., Schafer, D. A., Sept, D., Dominguez, R., \& Cooper, J. A. (2014). 
Capping protein regulators fine-tune actin assembly dynamics. Nature Reviews Molecular Cell Biology, 15(10), 677-689. https://doi.org/10.1038/nrm3869

Ennomani, H., Letort, G., Guérin, C., Martiel, J. L., Cao, W., Nédélec, F., ... Blanchoin, L. (2016). Architecture and Connectivity Govern Actin Network Contractility. Current Biology, 26(5), 616-626. https://doi.org/10.1016/j.cub.2015.12.069

Ertych, N., Stolz, A., Stenzinger, A., Weichert, W., Kaulfuß, S., Burfeind, P., ... Bastians, H. (2014). Increased microtubule assembly rates influence chromosomal instability in colorectal cancer cells. Nature Cell Biology, 16(8), 779-791.

https://doi.org/10.1038/ncb2994

Etienne-Manneville, S. (2013). Microtubules in Cell Migration. Annual Review of Cell and Developmental Biology, 29(1), 471-499. https://doi.org/10.1146/annurev-cellbio-101011155711

Fededa, J. P., \& Gerlich, D. W. (2012). Molecular control of animal cell cytokinesis. Nature Cell Biology, 14(5), 440-447. https://doi.org/10.1038/ncb2482

Fink, J., Carpi, N., Betz, T., Bétard, A., Chebah, M., Azioune, A., ... Piel, M. (2011). External forces control mitotic spindle positioning. Nature Cell Biology, 13(7), 771-778. https://doi.org/10.1038/ncb2269

Fischer-Friedrich, E., Hyman, A. A., Jülicher, F., Müller, D. J., \& Helenius, J. (2014). Quantification of surface tension and internal pressure generated by single mitotic cells. Scientific Reports, 4, 4-11. https://doi.org/10.1038/srep06213

Fodde, R., Smits, R., \& Clevers, H. (2001). APC, signal transduction and genetic instability in colorectal cancer. Nature Reviews Cancer, 1(1), 55-67. https://doi.org/10.1038/35094067

Foe, V. E., \& Von Dassow, G. (2008). Stable and dynamic microtubules coordinately shape the myosin activation zone during cytokinetic furrow formation. Journal of Cell Biology, 183(3), 457-470. https://doi.org/10.1083/jcb.200807128

Forbes, D. J., Travesa, A., Nord, M. S., \& Bernis, C. (2015). Nuclear transport factors: Global regulation of mitosis. Current Opinion in Cell Biology, 35, 78-90.

https://doi.org/10.1016/j.ceb.2015.04.012 
Forth, S., \& Kapoor, T. M. (2017). The mechanics of microtubule networks in cell division. Journal of Cell Biology, 216(6), 1525-1531. https://doi.org/10.1083/jcb.201612064

Fukasawa, K. (2007). Oncogenes and tumour suppressors take on centrosomes. Nature Reviews Cancer, 7(12), 911-924. https://doi.org/10.1038/nrc2249

Fukata, M., Watanabe, T., Noritake, J., Nakagawa, M., Yamaga, M., Kuroda, S., ... Kaibuchi, K. (2002). Rac1 and cdc42 cature microtubules through IQGAP1 and CLIP-170. Cell, 109(7), 873-885.

Ganem, N. J., Godinho, S. A., \& Pellman, D. (2009). A mechanism linking extra centrosomes to chromosomal instability. Nature, 460, 278-282. https://doi.org/10.1038/nature08136

Gao, Y., Dickerson, J. B., Guo, F., Zheng, J., \& Zheng, Y. (2004). Rational design and characterization of a Rac GTPase-specific small molecule inhibitor. PNAS, 101(20), 76187623. https://doi.org/10.1073/pnas.97.1.185

Gartel, A. L., Serfas, M. S., \& Tyner, A. L. (1996). p21-Negative Regulator of the Cell Cycle. Proceedings of the Society for Experimental Biology and Medicine, 213(2), 138-149. https://doi.org/10.3181/00379727-213-44046

Gartner, M., Sunder-Plassmann, N., Seiler, J., Utz, M., Vernos, I., Surrey, T., \& Giannis, A. (2005). Development and biological evaluation of potent and specific inhibitors of mitotic kinesin Eg5. ChemBioChem, 6(7), 1173-1177. https://doi.org/10.1002/cbic.200500005

Genth, H., Gerhard, R., Maeda, A., Amano, M., Kaibuchi, K., Aktories, K., \& Just, I. (2003). Entrapment of Rho ADP-ribosylated by Clostridium botulinum C3 exoenzyme in the Rhoguanine nucleotide dissociation inhibitor-1 complex. Journal of Biological Chemistry, 278(31), 28523-28527. https://doi.org/10.1074/jbc.M301915200

Gerlinger, M., \& Swanton, C. (2010). How Darwinian models inform therapeutic failure initiated by clonal heterogeneity in cancer medicine. British Journal of Cancer, 103(8), 1139-1143. https://doi.org/10.1038/sj.bjc.6605912

Glotzer, M. (1997). The mechanism and control of cytokinesis. Current Opinion in Cell Biology, 9(6), 815-823. https://doi.org/10.1016/S0955-0674(97)80082-8

Glotzer, M. (2005). The molecular requirements for cytokinesis. Science, 307(5716), 1735-1739. 
https://doi.org/10.1126/science.1096896

Goode, B. L., Drubin, D. G., \& Barnes, G. (2000). Functional cooperation between the microtubule and actin cytoskeletons. Current Opinion in Cell Biology, 12, 63-71. https://doi.org/10.1016/S0955-0674(99)00058-7

Goode, B. L., \& Eck, M. J. (2007). Mechanism and Function of Formins in the Control of Actin Assembly. Annual Review of Biochemistry, 76(1), 593-627. https://doi.org/10.1146/annurev.biochem.75.103004.142647

Goodson, H. V., Skube, S. B., Stalder, R., Valetti, C., Kreis, T. E., Morrison, E. E., \& Schroer, T. A. (2003). CLIP-170 interacts with dynactin complex and the APC-binding protein EB1 by different mechanisms. Cell Motility and the Cytoskeleton, 55(3), 156-173. https://doi.org/10.1002/cm.10114

Goshima, G., Mayer, M., Zhang, N., Stuurman, N., \& Vale, R. D. (2008). Augmin: A protein complex required for centrosome-independent microtubule generation within the spindle. Journal of Cell Biology, 181(3), 421-429. https://doi.org/10.1083/jcb.200711053

Green, R. A., Wollman, R., \& Kaplan, K. B. (2005). APC and EB1 Function Together in Mitosis to Regulate Spindle Dynamics and Chromosome Alignment. Molecular Biology of the Cell, 16(10), 4609-4622. https://doi.org/10.1091/mbc.E05

Guilluy, C., Garcia-Mata, R., \& Burridge, K. (2011). Rho protein crosstalk: Another social network? Trends in Cell Biology, 21(12), 718-726. https://doi.org/10.1016/j.tcb.2011.08.002

Gupta, K. K., Li, C., Duan, A., Alberico, E. O., Kim, O. V., Alber, M. S., \& Goodson, H. V. (2013). Mechanism for the catastrophe-promoting activity of the microtubule destabilizer Op18/stathmin. Proceedings of the National Academy of Sciences, 110(51), 20449-20454. https://doi.org/10.1073/pnas.1309958110

Hall, A. (1998). Rho GTpases and the actin cytoskeleton. Science, 279(5350), 509-514. https://doi.org/10.1126/science.279.5350.509

Halpain, S., \& Dehmelt, L. (2006). The MAP1 family of microtubule-associated proteins. Genome Biology, 7(6), 224. https://doi.org/10.1186/gb-2006-7-6-224

Hanna, S., \& El-Sibai, M. (2013). Signaling networks of Rho GTPases in cell motility. Cellular 
Signalling, 25(10), 1955-1961. https://doi.org/10.1016/j.cellsig.2013.04.009

Hannak, E., Kirkham, M., Hyman, A. A., \& Oegema, K. (2001). Aurora-A kinase is required for centrosome maturation in Caenorhabditis elegans. Journal of Cell Biology, 155(7), 11091115. https://doi.org/10.1083/jcb.200108051

Hao, Y., Du, Q., Chen, X., Zheng, Z., Balsbaugh, J. L., Maitra, S., .. MacAra, I. G. (2010). Par3 controls epithelial spindle orientation by aPKC-mediated phosphorylation of apical pins. Current Biology, 20(20), 1809-1818. https://doi.org/10.1016/j.cub.2010.09.032

Haviv, L., Gillo, D., Backouche, F., \& Bernheim-Groswasser, A. (2008). A Cytoskeletal Demolition Worker: Myosin II Acts as an Actin Depolymerization Agent. Journal of Molecular Biology, 375(2), 325-330. https://doi.org/10.1016/j.jmb.2007.09.066

Hayward, D., Metz, J., Pellacani, C., \& Wakefield, J. G. (2014). Synergy between Multiple Microtubule-Generating Pathways Confers Robustness to Centrosome-Driven Mitotic Spindle Formation. Developmental Cell, 28(1), 81-93.

https://doi.org/10.1016/j.devcel.2013.12.001

Heald, R., \& Khodjakov, A. (2015). Thirty years of search and capture: The complex simplicity of mitotic spindle assembly. Journal of Cell Biology, 211(6), 1103-1111. https://doi.org/10.1083/jcb.201510015

Heald, R., McLoughlin, M., \& McKeon, F. (1993). Human wee1 maintains mitotic timing by protecting the nucleus from cytoplasmically activated cdc2 kinase. Cell, 74(3), 463-474. https://doi.org/10.1016/0092-8674(93)80048-J

Heng, H. H., Bremer, S. W., Stevens, J. B., Horne, S. D., Liu, G., Abdallah, B. Y., .. Ye, C. J. (2013). Chromosomal instability (CIN): What it is and why it is crucial to cancer evolution. Cancer and Metastasis Reviews, 32(3-4), 325-340. https://doi.org/10.1007/s10555-0139427-7

Henty-Ridilla, J. L., Rankova, A., Eskin, J. A., Kenny, K., \& Goode, B. L. (2016). Accelerated actin filament polymerization from microtubule plus ends. Science, 352(6288), 1004-1010.

Hess, H. A., Röper, J. C., Grill, S. W., \& Koelle, M. R. (2004). RGS-7 completes a receptorindependent heterotrimeric $\mathrm{G}$ protein cycle to asymmetrically regulate mitotic spindle positioning in C. elegans. Cell, 119(2), 209-218. https://doi.org/10.1016/j.cell.2004.09.025 
Higgs, H. N. (2005). Formin proteins: A domain-based approach. Trends in Biochemical Sciences, 30(6), 342-353. https://doi.org/10.1016/j.tibs.2005.04.014

Higgs, H. N., \& Peterson, K. J. (2005). Phylogenetic Analysis of the Formin Homology 2 Domain. Molecular Biology of the Cell, 16(1), 1-13. https://doi.org/10.1091/mbc.E04

Hohmann, \& Dehghani. (2019). The Cytoskeleton-A Complex Interacting Meshwork. Cells, 8(4), 362. https://doi.org/10.3390/cells8040362

Howard, J., \& Hyman, A. A. (2009). Growth, fluctuation and switching at microtubule plus ends. Nature Reviews Molecular Cell Biology, 10, 569-574. https://doi.org/10.1038/nrm2713

Hunter, A. W., Caplow, M., Coy, D. L., Hancock, W. O., Diez, S., Wordeman, L., \& Howard, J. (2003). The kinesin-related protein MCAK is a microtubule depolymerase that forms an ATP-hydrolyzing complex at microtubule ends. Molecular Cell, 11(2), 445-457. https://doi.org/10.1016/S1097-2765(03)00049-2

Jallepalli, P. V., Waizenegger, I. C., Bunz, F., Langer, S., Speicher, M. R., Peters, J. M., ... Lengauer, C. (2001). Securin is required for chromosomal stability in human cells. Cell, 105(4), 445-457. https://doi.org/10.1016/S0092-8674(01)00340-3

Jones, S., Zhang, X., Parsons, D. W., Lin, J. C. H., Leary, R. J., Angenendt, P., ... Kinzler, K. W. (2008). Core signaling pathways in human pancreatic cancers revealed by global genomic analyses. Science, 321(5897), 1801-1806. https://doi.org/10.1126/science.1164368

Jordan, M. A., \& Wilson, L. (2004). Microtubules as a target for anticancer drugs. Nature Reviews Cancer, 4, 253-265. https://doi.org/10.1038/nrc1317

Joyce, D., Bouzahzah, B., Fu, M., Albanese, C., D'Amico, M., Steer, J., ... Pestell, R. G. (1999). Integration of Rac-dependent regulation of cyclin D1 transcription through a nuclear factorkB-dependent pathway. Journal of Biological Chemistry, 274(36), 25245-25249. https://doi.org/10.1074/jbc.274.36.25245

Kamasaki, T., O’Toole, E., Kita, S., Osumi, M., Usukura, J., Mclntosh, R. R., \& Goshima, G. (2013). Augmin-dependent microtubule nucleation at microtubule walls in the spindle. Journal of Cell Biology, 202(1), 25-32. https://doi.org/10.1083/jcb.201304031

Kardon, J. R., \& Vale, R. D. (2009). Regulators of the cytoplasmic dynein motor. Nature Reviews 
Molecular Cell Biology, 10(12), 854-865. https://doi.org/10.1038/nrm2804

Kaverina, I., \& Straube, A. (2011). Regulation of cell migration by dynamic microtubules.

Seminars in Cell and Developmental Biology, 22(9), 968-974.

https://doi.org/10.1016/j.semcdb.2011.09.017

Kelly, A. E., Sampath, S. C., Maniar, T. A., Woo, E. M., Chait, B. T., \& Funabiki, H. (2007).

Chromosomal Enrichment and Activation of the Aurora B Pathway Are Coupled to Spatially Regulate Spindle Assembly. Developmental Cell, 12(1), 31-43.

https://doi.org/10.1016/j.devcel.2006.11.001

Kirschner, M., \& Mitchison, T. (1986). Beyond self-assembly: From microtubules to morphogenesis. Cell, 45(3), 329-342. https://doi.org/10.1016/0092-8674(86)90318-1

Kiyomitsu, T., \& Cheeseman, I. M. (2012). Chromosome-and spindle-pole-derived signals generate an intrinsic code for spindle position and orientation. Nature Cell Biology, 14(3), 311-317. https://doi.org/10.1038/ncb2440

Kohn, K. W., Zeeberg, B. R., Reinhold, W. C., Sunshine, M., Luna, A., \& Pommier, Y. (2012). Gene expression profiles of the $\mathrm{NCl}-60$ human tumor cell lines define molecular interaction networks governing cell migration processes. PLOS ONE, 7(5). https://doi.org/10.1371/journal.pone.0035716

Kops, G. J. P. L., Foltz, D. R., \& Cleveland, D. W. (2004). Lethality to human cancer cells through massive chromosome loss by inhibition of the mitotic checkpoint. Proceedings of the National Academy of Sciences of the United States of America, 101(23), 8699-8704. https://doi.org/10.1073/pnas.0401142101

Kotak, S., Busso, C., \& Gönczy, P. (2012). Cortical dynein is critical for proper spindle positioning in human cells. Journal of Cell Biology, 199(1), 97-110.

https://doi.org/10.1083/jcb.201203166

Kovács, M., Tóth, J., Hetényi, C., Málnási-Csizmadia, A., \& Seller, J. R. (2004). Mechanism of blebbistatin inhibition of myosin II. Journal of Biological Chemistry, 279(34), 35557-35563. https://doi.org/10.1074/jbc.M405319200

Krendel, M., Zenke, F. T., \& Bokoch, G. M. (2002). Nucleotide exchange factor GEF-H1 mediates cross-talk between microtubules and the actin cytoskeleton. Nature Cell Biology, 
4(4), 294-301. https://doi.org/10.1038/ncb773

Kunda, P., \& Baum, B. (2009). The actin cytoskeleton in spindle assembly and positioning. Trends in Cell Biology, 19(4), 174-179. https://doi.org/10.1016/j.tcb.2009.01.006

Kunda, P., Pelling, A. E., Liu, T., \& Baum, B. (2008). Moesin Controls Cortical Rigidity, Cell Rounding, and Spindle Morphogenesis during Mitosis. Current Biology, 18(2), 91-101. https://doi.org/10.1016/j.cub.2007.12.051

Kuroda, S., Fukata, M., Nakagawa, M., \& Kaibuchi, K. (1999). Cdc42, Rac1, and their effector IQGAP1 as molecular switches for cadherin-mediated cell-cell adhesion. Biochemical and Biophysical Research Communications, 262(1), 1-6.

https://doi.org/10.1006/bbrc.1999.1122

Kwon, M., Bagonis, M., Danuser, G., \& Pellman, D. (2015). Direct Microtubule-Binding by Myosin-10 Orients Centrosomes toward Retraction Fibers and Subcortical Actin Clouds. Developmental Cell, 34(3), 323-337. https://doi.org/10.1016/j.devcel.2015.06.013

Lancaster, O., LeBerre, M., Dimitracopoulos, A., Bonazzi, D., Zlotek-Zlotkiewicz, E., Picone, R., ... Baum, B. (2013). Mitotic Rounding Alters Cell Geometry to Ensure Efficient Bipolar Spindle Formation. Developmental Cell, 25(3), 270-283.

https://doi.org/10.1016/j.devcel.2013.03.014

Lane, H. A., \& Nigg, E. A. (1996). Antibody microinjection reveals an essential role for human polo-like kinase 1 (Plk1) in the functional maturation of mitotic centrosomes. Journal of Cell Biology, $135(6$ II), 1701-1713. https://doi.org/10.1083/jcb.135.6.1701

Lane, J., Martin, T. A., Mansel, R. E., \& Jiang, W. G. (2008). The expression and prognostic value of the guanine nucleotide exchange factors (GEFs) Trio, Vav1 and TIAM-1 in human breast cancer. International Seminars in Surgical Oncology, 5, 1-7. https://doi.org/10.1186/1477-7800-5-23

Lane, J., Martin, T. A., Watkins, G., Mansel, R. E., \& Jiang, W. G. (2008). The expression and prognostic value of ROCK I and ROCK II and their role in human breast cancer. International Journal of Oncology, 33(3), 585-593. https://doi.org/10.3892/ijo

Laskey, R. A., Fairman, M. P., \& Blow, J. J. (1989). S Phase of the Cell Cycle. Science, 246(4930), 609-614. 
Lawler, S. (2004). Microtubule dynamics: If you need a shrink try stathmin/Op18. Current Biology, 8(6), R212-R214. https://doi.org/10.1016/s0960-9822(98)70128-9

Lawson, C. D., \& Burridge, K. (2014). The on-off relationship of Rho and Rac during integrinmediated adhesion and cell migration. Small GTPases, 5(MAR). https://doi.org/10.4161/sgtp.27958

Lázaro-Diéguez, F., Ispolatov, I., \& Müsch, A. (2015). Cell shape impacts on the positioning of the mitotic spindle with respect to the substratum. Molecular Biology of the Cell, 26(7), 1286-1295. https://doi.org/10.1091/mbc.e14-08-1330

Lecland, N., \& Lüders, J. (2014). The dynamics of microtubule minus ends in the human mitotic spindle. Nature Cell Biology, 16(8), 770-778. https://doi.org/10.1038/ncb2996

Lee, A. J. X., Endesfelder, D., Rowan, A. J., Walther, A., Birkbak, N. J., Futreal, P. A., ... Swanton, C. (2011). Chromosomal instability confers intrinsic multidrug resistance. Cancer Research, 71(5), 1858-1870. https://doi.org/10.1158/0008-5472.CAN-10-3604

Lee, K., \& Rhee, K. (2011). PLK1 phosphorylation of pericentrin initiates centrosome maturation at the onset of mitosis. Journal of Cell Biology, 195(7), 1093-1101. https://doi.org/10.1083/jcb.201106093

Lee, S. H., \& Dominguez, R. (2010). Regulation of Actin Cytoskeleton Dynamics in Cells. Mol. Cells, 29, 311-325. https://doi.org/10.1007/s10059-010-0053-8

Lengauer, C., Kinzler, K. W., \& Vogelstein, B. (1997). Genetic instability in colorectal cancers. Nature, 386, 623-627. https://doi.org/10.1038/386623a0

Levayer, R., \& Lecuit, T. (2012). Biomechanical regulation of contractility: Spatial control and dynamics. Trends in Cell Biology, 22(2), 61-81. https://doi.org/10.1016/j.tcb.2011.10.001

Leve, F., \& Morgado-Díaz, J. A. (2012). Rho GTPase signaling in the development of colorectal cancer. Journal of Cellular Biochemistry, 113(8), 2549-2559.

https://doi.org/10.1002/jcb.24153

Lewkowicz, E., Herit, F., Clainche, C. Le, Bourdoncle, P., Perez, F., \& Niedergang, F. (2008). The microtubule-binding protein CLIP-170 coordinates mDia1 and actin reorganization during CR3-mediated phagocytosis. Journal of Cell Biology, 183(7), 1287-1298. 
https://doi.org/10.1083/jcb.200807023

Li, F., \& Higgs, H. N. (2003). The Mouse Formin mDia1 Is a Potent Actin Nucleation Factor Regulated by Autoinhibition. Current Biology, 13(15), 1335-1340. https://doi.org/10.1016/S

Li, F., \& Higgs, H. N. (2005). Dissecting requirements for auto-inhibition of actin nucleation by the formin, mDia1. Journal of Biological Chemistry, 280(8), 6986-6992. https://doi.org/10.1074/jbc.M411605200

Liu, D., Vader, G., Vromans, M. J. M., Lampson, M. A., \& Lens, S. M. A. (2009). Sensing Chromosome Bi-Orientation Kinase from Kinetochore Substrates. Science, 323(5919), 1350-1353. https://doi.org/10.1126/science.1167000

Liu, J., \& Han, R. (2015). The Evolution of Microtubule End-Binding Protein 1 (EB1) and Roles in Regulating Microtubule Behavior. American Journal of Plant Sciences, 6, 2114-2121. https://doi.org/10.4236/ajps.2015.613212

Lizárraga, F., Poincloux, R., Romão, M., Montagnac, G., Le Dez, G., Bonne, I., ... Chavrier, P. (2009). Diaphanous-related formins are required for invadopodia formation and invasion of breast tumor cells. Cancer Research, 69(7), 2792-2800. https://doi.org/10.1158/00085472.CAN-08-3709

Louie, R. K., Bahmanyar, S., Siemers, K. A., Votin, V., Chang, P., Stearns, T., ... Barth, A. I. M. (2004). Adenomatous polyposis coli and EB1 localize in close proximity of the mother centriole and EB1 is a functional component of centrosomes. Journal of Cell Science, 117(7), 1117-1128. https://doi.org/10.1242/jcs.00939

Lüders, J., \& Stearns, T. (2007). Microtubule-organizing centres: a re-evaluation. Nature Reviews Molecular Cell Biology, 8(2), 161-167.

Luxenburg, C., Amalia Pasolli, H., Williams, S. E., \& Fuchs, E. (2011). Developmental roles for Srf, cortical cytoskeleton and cell shape in epidermal spindle orientation. Nature Cell Biology, 13(3), 203-214. https://doi.org/10.1038/ncb2163

Machicoane, M., de Frutos, C. A., Fink, J., Rocancourt, M., Lombardi, Y., Gare, S., ... Echard, A. (2014). SLK-dependent activation of ERMs controls LGN-NuMA localization and spindle orientation. Journal of Cell Biology, 205(6), 791-799. https://doi.org/10.1083/jcb.201401049 
Mackay, D. J. G., \& Hall, A. (1998). Rho GTPases. THE JOURNAL OF BIOLOGICAL CHEMISTRY, 273(33), 20685-20688. Retrieved from http://www.jbc.org/

Maddox, A. S., \& Burridge, K. (2003). RhoA is required for cortical retraction and rigidity during mitotic cell rounding. Journal of Cell Biology, 160(2), 255-165.

https://doi.org/10.1083/jcb.200207130

Maekawa, M., Ishizaki, T., Boku, S., Watanabe, N., Fujita, A., Iwamatsu, A., .. Narumiya, S. (1999). Signaling from Rho to the Actin Cytoskeleton Through Protein Kinases ROCK and LIM-kinase. Science, 285(5429), 895-898.

Maiato, H., DuLeca, J., Salmon, E. D., \& Earnshaw, W. C. (2004). The dynamic kinetochoremicrotubule interface. Journal of Cell Science, 117(23), 5461-5477. https://doi.org/10.1242/jcs.01536

Maier, B., Kirsch, M., Anderhub, S., Zentgraf, H., \& Krämer, A. (2013). The novel actin/focal adhesion-associated protein MISP is involved in mitotic spindle positioning in human cells. Cell Cycle, 12(9), 1457-1471. https://doi.org/10.4161/cc.24602

Malumbres, M., \& Barbacid, M. (2001). To cycle or not to cycle: a critical decision in cancer. Nature Reviews Cancer, 1(3), 222-231. https://doi.org/10.1038/35106065

Malumbres, M., \& Barbacid, M. (2005). Mammalian cyclin-dependent kinases. Trends in Biochemical Sciences, 30(11), 630-641. https://doi.org/10.1016/j.tibs.2005.09.005

Malumbres, M., \& Barbacid, M. (2009). Cell cycle, CDKs and cancer: A changing paradigm. Nature Reviews Cancer, 9(3), 153-166. https://doi.org/10.1038/nrc2602

Mandato, C. A., Benink, H. A., \& Bement, W. M. (2000). Microtubule-actomyosin interactions in cortical flow and cytokinesis. Cell Motility and the Cytoskeleton, 45(2), 87-92. https://doi.org/10.1002/(SICI)1097-0169(200002)45:2<87::AID-CM1>3.0.CO;2-0

Mann, B. J., \& Wadsworth, P. (2019). Kinesin-5 Regulation and Function in Mitosis. Trends in Cell Biology, 29(1), 66-79. https://doi.org/10.1016/j.tcb.2018.08.004

Mardilovich, K., Olson, M. F., \& Baugh, M. (2012). Targeting Rho GTPase signaling for cancer therapy. Future Oncology, 8(2), 165-177. https://doi.org/10.2217/fon.11.143 
Marei, H., \& Malliri, A. (2017). GEFs: Dual regulation of Rac1 signaling. Small GTPases, 8(2), 90-99. https://doi.org/10.1080/21541248.2016.1202635

Margolis, R. L., \& Wilson, L. (1998). Microtubule treadmilling: What goes around comes around. BioEssays, 20(10), 830-836. https://doi.org/10.1002/(SICI)15211878(199810)20:10<830::AID-BIES8>3.0.CO;2-N

Martin-Belmonte, F., \& Perez-Moreno, M. (2012). Epithelial cell polarity, stem cells and cancer. Nature Reviews Cancer, 12(1), 23-38. https://doi.org/10.1038/nrc3169

Matsumura, F., \& Hartshorne, D. J. (2008). Myosin phosphatase target subunit: Many roles in cell function. Biochemical and Biophysical Research Communications, 369(1), 149-156. https://doi.org/10.1016/j.bbrc.2007.12.090

Mazumdar, M., \& Misteli, T. (2005). Chromokinesins: Multitalented players in mitosis. Trends in Cell Biology, 15(7), 349-355. https://doi.org/10.1016/j.tcb.2005.05.006

McGranahan, N., Burrell, R. A., Endesfelder, D., Novelli, M. R., \& Swanton, C. (2012). Cancer chromosomal instability: Therapeutic and diagnostic challenges. EMBO Reports, 13(6), 528-538. https://doi.org/10.1038/embor.2012.61

Meraldi, P. (2016). Centrosomes in spindle organization and chromosome segregation: a mechanistic view. Chromosome Research, 24(1), 19-34. https://doi.org/10.1007/s10577015-9508-2

Merdes, A., Ramyar, K., Vechio, J. D., \& Cleveland, D. W. (1996). A complex of NuMA and cytoplasmic dynein is essential for mitotic spindle assembly. Cell, 87(3), 447-458. https://doi.org/10.1016/S0092-8674(00)81365-3

Meunier, S., \& Vernos, I. (2016). Acentrosomal Microtubule Assembly in Mitosis: The Where, When, and How. Trends in Cell Biology, 26(2), 80-87. https://doi.org/10.1016/j.tcb.2015.09.001

Mimori-Kiyosue, Y., Grigoriev, I., Lansbergen, G., Sasaki, H., Matsui, C., Severin, F., ... Akhmanova, A. (2005). CLASP1 and CLASP2 bind to EB1 and regulate microtubule plusend dynamics at the cell cortex. Journal of Cell Biology. https://doi.org/10.1083/jcb.200405094 
Mitchison, T. J. (1992). Actin based motility on retraction fibers in mitotic PtK2 cells. Cell Motility and the Cytoskeleton, 22(2), 135-151. https://doi.org/10.1002/cm.970220207

Mitchison, T., \& Kirschner, M. (1984). Dynamic instability of microtubule growth. Nature, 312(5991), 237-242. https://doi.org/10.1038/312237a0

Mitsushima, M., Aoki, K., Ebisuya, M., Matsumura, S., Yamamoto, T., Matsuda, M., ... Nishida, E. (2010). Revolving movement of a dynamic cluster of actin filaments during mitosis. Journal of Cell Biology, 191(3), 453-462. https://doi.org/10.1083/jcb.201007136

Montalvo-Ortiz, B. L., Castillo-Pichardo, L., Hernández, E., Humphries-Bickley, T., De La MotaPeynado, A., Cubano, L. A., ... Dharmawardhane, S. (2012). Characterization of EHop016, novel small molecule inhibitor of Rac GTPase. Journal of Biological Chemistry, 287(16), 13228-13238. https://doi.org/10.1074/jbc.M111.334524

Morgan, D. O. (1997). CYCLIN-DEPENDENT KINASES: Engines, Clocks, and Microprocessors. Annual Review of Cell and Developmental Biology, 13(1), 261-291. https://doi.org/10.1146/annurev.cellbio.13.1.261

Morin, X., \& Bellaïche, Y. (2011). Mitotic Spindle Orientation in Asymmetric and Symmetric Cell Divisions during Animal Development. Developmental Cell, 21(1), 102-119. https://doi.org/10.1016/j.devcel.2011.06.012

Morton, W. M., Ayscough, K. R., \& Mclaughlin, P. J. (2000). Latrunculin alters the actinmonomer subunit interface to prevent polymerization. Nature Cell Biology, 2, 376-378. https://doi.org/10.1038/35014075

Mourey, L., Agard, D. a., Kollman, J. M., \& Merdes, A. (2011). Microtubule nucleation by Ytubulin complexes. Nature Reviews Molecular Cell Biology, 12(11), 709-721. https://doi.org/10.1038/nrm3209

Mullins, R. D., Heuser, J. A., \& Pollard, T. D. (1998). The interaction of Arp2/3 complex with actin: Nucleation, high affinity pointed end capping, and formation of branching networks of filaments. Proceedings of the National Academy of Sciences, 95(11), 6181-6186. https://doi.org/10.1073/pnas.95.11.6181

Musacchio, A. (2015). The Molecular Biology of Spindle Assembly Checkpoint Signaling Dynamics. Current Biology, 25(20), R1002-R1018. 
https://doi.org/10.1016/j.cub.2015.08.051

Musgrove, E. A. (2006). Cyclins: Roles in mitogenic signaling and oncogenic transformation. Growth Factors, 24(1), 13-19. https://doi.org/10.1080/08977190500361812

Nam, H. J., Naylor, R. M., \& van Deursen, J. M. (2015). Centrosome dynamics as a source of chromosomal instability. Trends in Cell Biology, 25(2), 65-73.

https://doi.org/10.1016/j.tcb.2014.10.002

Nam, H. J., \& van Deursen, J. M. (2014). Cyclin B2 and p53 control proper timing of centrosome separation. Nature Cell Biology, 16(6), 538-549. https://doi.org/110.1016/j.bbi.2017.04.008

Narumiya, S., Tanji, M., \& Ishizaki, T. (2009). Rho signaling, ROCK and mDia1, in transformation, metastasis and invasion. Cancer and Metastasis Reviews, 28, 65-76. https://doi.org/10.1007/s10555-008-9170-7

Nigg, E. A. (1995). Cyclin-dependent protein kinases: key regulators of the eukaryotic cell cycle. BioEssays, 17(6), 471-480.

Nigg, E. A. (2001). Mitotic kinases as regulators of cell division and its checkpoints. Nature Reviews Molecular Cell Biology, 2(1), 21-32. Retrieved from papers3://publication/uuid/574A8FA8-56D5-47A4-9664-91D031912446

Noatynska, A., Gotta, M., \& Meraldi, P. (2012). Mitotic spindle (DIS)orientation and DISease: Cause or consequence? Journal of Cell Biology, 199(7), 1025-1035. https://doi.org/10.1083/jcb.201209015

Nobes, C. D., \& Hall, A. (1999). Rho GTPases control polarity, protrusion, and adhesion during cell movement. Journal of Cell Biology, 144(6), 1235-1244. https://doi.org/10.1083/jcb.144.6.1235

Nolen, B. J., Tomasevic, N., Russell, A., Pierce, D. W., Jia, Z., McCormick, C. D., ... Pollard, T. D. (2009). Characterization of two classes of small molecule inhibitors of Arp2/3 complex. Nature. https://doi.org/10.1038/nature08231

Norbury, C., \& Nurse, P. (1992). Animal Cell Cycles and Their Control. Annual Review of Biochemistry, 61(1), 441-470. https://doi.org/10.1146/annurev.biochem.61.1.441 
Ohi R, \& Zanic M. (2016). Ahead of the Curve: New Insights into Microtubule Dynamics. F1000Research, 5(314). https://doi.org/10.12688/f1000research.7439.1

Okumura, M., Natsume, T., Kanemaki, M. T., \& Kiyomitsu, T. (2018). Dynein-dynactin-NuMA clusters generate cortical spindle-pulling forces as a multiarm ensemble. ELife, 7, 1-24. https://doi.org/10.7554/eLife.36559

Olson, M. F., Paterson, H. F., \& Marshall, C. J. (1998). Signals from Ras and Rho GTPases interact to regulate expression of p21Waf1/Cip1. Nature, 394(6690), 295-299. https://doi.org/10.1038/255243a0

Orr, B., Godek, K. M., \& Compton, D. A. (2015). Aneuploidy. Current Biology, 25(13), R523-548. https://doi.org/10.1016/B978-0-12-374984-0.00064-4

Parsons, D. W., Jones, S., Zhang, X., Lin, J. C. H., Leary, R. J., Angenendt, P., ... Kinzler, K. W. (2008). An integrated genomic analysis of human glioblastoma multiforme. Science, 321(5897), 1807-1812. https://doi.org/10.1126/science.1164382

Payapilly, A., \& Malliri, A. (2018). Compartmentalisation of RAC1 signalling. Current Opinion in Cell Biology, 54, 50-56. https://doi.org/10.1016/j.ceb.2018.04.009

Peters, J.-M. (2002). The Anaphase-Promoting Complex. Molecular Cell, 9(5), 931-943. https://doi.org/10.1016/s1097-2765(02)00540-3

Peters, J. M., Tedeschi, A., \& Schmitz, J. (2008). The cohesin complex and its roles in chromosome biology. Genes and Development, 22(3089), 3089-3114. https://doi.org/10.1101/gad.1724308

Peyre, E., Jaouen, F., Saadaoui, M., Haren, L., Merdes, A., Durbec, P., \& Morin, X. (2011). A lateral belt of cortical LGN and NuMA guides mitotic spindle movements and planar division in neuroepithelial cells. Journal of Cell Biology, 193(1), 141-154.

https://doi.org/10.1083/jcb.201101039

Pollard, T. D. (2007). Regulation of Actin Filament Assembly by Arp2/3 Complex and Formins. Annual Review of Biophysics and Biomolecular Structure, 36(1), 451-477. https://doi.org/10.1146/annurev.biophys.35.040405.101936

Pollard, T. D. (2016). Actin and Actin-Binding Proteins. Cold Spring Harbor Perspectives in 
Biology, 8(8), a018226. https://doi.org/10.1101/cshperspect.a018226

Powell, S. M., Zilz, N., Beazer-Barclay, Y., Bryan, T. M., Hamilton, S. R., Thibodeau, S. N., .. Kinzler, K. W. (1992). APC mutations occur early during colorectal tumorigenesis. Nature, 359(6392), 235-237. https://doi.org/10.1038/359235a0

Prosser, S. L., \& Pelletier, L. (2017). Mitotic spindle assembly in animal cells: A fine balancing act. Nature Reviews Molecular Cell Biology, 18(3), 187-201.

https://doi.org/10.1038/nrm.2016.162

Pruyne, D., Evangelista, M., Yang, C., Bi, E., Zigmond, S., Bretscher, A., \& Boone, C. (2016). Role of Formins in Actin Assembly : Nucleation and Barbed-End Association. Science, 297(5581), 612-615.

Quyn, A. J., Appleton, P. L., Carey, F. A., Steele, R. J. C., Barker, N., Clevers, H., .. Näthke, I. S. (2010). Spindle Orientation Bias in Gut Epithelial Stem Cell Compartments Is Lost in Precancerous Tissue. Cell Stem Cell, 6(2), 175-181.

https://doi.org/10.1016/j.stem.2009.12.007

Raaijmakers, J. A., Van Heesbeen, R. G. H. P., Meaders, J. L., Geers, E. F., Fernandez-Garcia, B., Medema, R. H., \& Tanenbaum, M. E. (2012). Nuclear envelope-associated dynein drives prophase centrosome separation and enables Eg5-independent bipolar spindle formation. EMBO Journal, 31(21), 4179-4190. https://doi.org/10.1038/emboj.2012.272

Ramanathan, S. P., Helenius, J., Stewart, M. P., Cattin, C. J., Hyman, A. A., \& Muller, D. J. (2015). Cdk1-dependent mitotic enrichment of cortical myosin II promotes cell rounding against confinement. Nature Cell Biology, 17(2), 148-159. https://doi.org/10.1038/ncb3098

Remmerbach, T. W., Wottawah, F., Dietrich, J., Lincoln, B., Wittekind, C., \& Guck, J. (2009). Oral cancer diagnosis by mechanical phenotyping. Cancer Research, 69(5), 1728-1732. https://doi.org/10.1158/0008-5472.CAN-08-4073

Ren, X., Kiosses, W. B., \& Schwartz, M. A. (1999). Regulation of the small GTP-binding protein Rho by cell adhesion and the cytoskeleton. The EMBO Journal, 18(3), 578-585.

Reymann, A. C., Boujemaa-Paterski, R., Martiel, J. L., Guérin, C., Cao, W., Chin, H. F., ... Blanchoin, L. (2012). Actin network architecture can determine myosin motor activity. Science, 336(6086), 1310-1314. https://doi.org/10.1126/science.1221708 
Ricke, R. M., van Ree, J. H., \& van Deursen, J. M. (2008). Whole chromosome instability and cancer: a complex relationship. Trends in Genetics, 24(9), 457-466.

https://doi.org/10.1016/j.tig.2008.07.002

Rieder, C. L., Cole, R. W., Khodjakov, A., \& Sluder, G. (1995). The checkpoint delaying anaphase in response to chromosome monoorientation is mediated by an inhibitory signal produced by unattached kinetochores. Journal of Cell Biology, 130(4), 941-948. https://doi.org/10.1083/jcb.130.4.941

Rieder, C. L., \& Salmon, E. D. (1994). Motile kinetochores and polar ejection forces dictate chromosome position on the vertebrate mitotic spindle. The Journal of Cell Biology, 124(3), 223-233. https://doi.org/10.1083/jcb.124.3.223

Rizvi, S. A., Neidt, E. M., Cui, J., Feiger, Z., Skau, C. T., Gardel, M. L., ... Kovar, D. R. (2009). Identification and Characterization of a Small Molecule Inhibitor of Formin-Mediated Actin Assembly. Chemistry and Biology, 16(11), 1158-1168.

https://doi.org/10.1016/j.chembiol.2009.10.006

Rosenblatt, J., Cramer, L. P., Baum, B., \& McGee, K. M. (2004). Myosin II-dependent cortical movement is required for centrosome separation and positioning during mitotic spindle assembly. Cell, 117(3), 361-372. https://doi.org/10.1016/S0092-8674(04)00341-1

Rotty, J. D., Wu, C., \& Bear, J. E. (2013). New insights into the regulation and cellular functions of the ARP2/3 complex. Nature Reviews Molecular Cell Biology, 14(1), 7-12. https://doi.org/10.1038/nrm3492

Rouiller, I., Xu, X. P., Amann, K. J., Egile, C., Nickell, S., Nicastro, D., ... Hanein, D. (2008). The structural basis of actin filament branching by the Arp2/3 complex. Journal of Cell Biology, 180(5), 887-895. https://doi.org/10.1083/jcb.200709092

Roylance, R., Endesfelder, D., Gorman, P., Burrell, R. A., Sander, J., Tomlinson, I., ... Swanton, C. (2011). Relationship of extreme chromosomal instability with long-term survival in a retrospective analysis of primary breast cancer. Cancer Epidemiology Biomarkers and Prevention, 20(10), 2183-2194. https://doi.org/10.1158/1055-9965.EPI-11-0343

Safer, D., \& Nachmias, V. T. (1994). Beta thymosins as actin binding peptides. BioEssays, 16(7), 473-479. https://doi.org/10.1002/bies.950160706 
Sagot, I., Rodal, A. A., Moseley, J., Goode, B. L., \& Pellman, D. (2002). An actin nucleation mechanism mediated by Bni1 and profilin. Nature Cell Biology, 4(8), 626-631. https://doi.org/10.1038/ncb834

Sahai, E., \& Marshall, C. J. (2002). Rho-GTPases and Cancer. Cancer, 2(February). https://doi.org/10.1038/nrc725

Salbreux, G., Charras, G., \& Paluch, E. (2012). Actin cortex mechanics and cellular morphogenesis. Trends in Cell Biology, 22(10), 536-545.

https://doi.org/10.1016/j.tcb.2012.07.001

Salhia, B., Tran, N. L., Chan, A., Wolf, A., Nakada, M., Rutka, F., ... Rutka, J. T. (2008). The guanine nucleotide exchange factors Trio, Ect2, and Vav3 mediate the invasive behavior of glioblastoma. American Journal of Pathology, 173(6), 1828-1838.

https://doi.org/10.2353/ajpath.2008.080043

Sampath, S. C., Ohi, R., Leismann, O., Salic, A., Pozniakovski, A., \& Funabiki, H. (2004). The chromosomal passenger complex is required for chromatin-induced microtubule stabilization and spindle assembly. Cell, 118(2), 187-202.

https://doi.org/10.1016/j.cell.2004.06.026

Sánchez-Huertas, C., \& Lüders, J. (2015). The augmin connection in the geometry of microtubule networks. Current Biology, 25(7), R294-R299.

https://doi.org/10.1016/j.cub.2015.02.006

Sánchez, I., \& Dynlacht, B. D. (2005). New insights into cyclins, CDKs, and cell cycle control. Seminars in Cell and Developmental Biology, 16(3), 311-321.

https://doi.org/10.1016/j.semcdb.2005.02.007

Santaguida, S., Richardson, A., Iyer, D. R., M'Saad, O., Zasadil, L., Knouse, K. A., ... Amon, A. (2017). Chromosome Mis-segregation Generates Cell-Cycle-Arrested Cells with Complex Karyotypes that Are Eliminated by the Immune System. Developmental Cell, 41(6), 638651.e5. https://doi.org/10.1016/j.devcel.2017.05.022

Schirenbeck, A., Bretschneider, T., Arasada, R., Schleicher, M., \& Faix, J. (2005). The Diaphanous-related formin dDia2 is required for the formation and maintenance of filopodia. Nature Cell Biology, 7(6), 619-625. https://doi.org/10.1038/ncb1266 
Schmidt, A., \& Hall, M. N. (1998). SIGNALING TO THE ACTIN CYTOSKELETON. Annu. Rev. Cell Dev. Biol, 14(1), 305-343. Retrieved from www.annualreviews.org

Schoenwaelder, S. M., Petch, L. A., Williamson, D., Shen, R., Feng, G. S., \& Burridge, K. (2000). The protein tyrosine phosphatase Shp-2 regulates RhoA activity. Current Biology, 10(23), 1523-1526. https://doi.org/10.1016/S0960-9822(00)00831-9

Scholey, J. M., Brust-mascher, I., \& Mogilner, A. (2003). Cell division. Nature, 422(6933), 746752.

Schuh, M., \& Ellenberg, J. (2008). A New Model for Asymmetric Spindle Positioning in Mouse Oocytes. Current Biology, 18(24), 1986-1992. https://doi.org/10.1016/j.cub.2008.11.022

Seldin, L., Muroyama, A., \& Lechler, T. (2016). NuMA-microtubule interactions are critical for spindle orientation and the morphogenesis of diverse epidermal structures. ELife, 5(JANUARY2016), 1-18. https://doi.org/10.7554/eLife.12504.001

Sept, D., \& McCammon, J. A. (2001). Thermodynamics and kinetics of actin filament nucleation. Biophysical Journal, 81(2), 667-674. https://doi.org/10.1016/S0006-3495(01)75731-1

Sheltzer, J. M. (2013). A transcriptional and metabolic signature of primary aneuploidy is present in chromosomally unstable cancer cells and informs clinical prognosis. Cancer Research, 73(21), 6401-6412. https://doi.org/10.1158/0008-5472.CAN-13-0749

Silk, A. D., Zasadil, L. M., Holland, A. J., Vitre, B., Cleveland, D. W., \& Weaver, B. A. (2013). Chromosome missegregation rate predicts whether aneuploidy will promote or suppress tumors. Proceedings of the National Academy of Sciences, 110(44), E4134-E4141. https://doi.org/10.1073/pnas.1317042110

Silkworth, W. T., \& Cimini, D. (2012). Transient defects of mitotic spindle geometry and chromosome segregation errors. Cell Division, 7(19), 1-8. https://doi.org/10.1186/17471028-7-19

Sivaramakrishnan, S., Ashley, E., Leinwand, L., \& Spudich, J. A. (2009). Insights into human $\beta$ cardiac myosin function from single molecule and single cell studies. Journal of Cardiovascular Translational Research, 2(4), 426-440. https://doi.org/10.1007/s12265-0099129-2 
Smith, E., Hégarat, N., Vesely, C., Roseboom, I., Larch, C., Streicher, H., ... Hochegger, H. (2011). Differential control of Eg5-dependent centrosome separation by Plk1 and Cdk1. EMBO Journal, 30(11), 2233-2245. https://doi.org/10.1038/emboj.2011.120

Solomon, D. A., Kim, J. S., Bondaruk, J., Shariat, S. F., Wang, Z. F., Elkahloun, A. G., ... Waldman, T. (2013). Frequent truncating mutations of STAG2 in bladder cancer. Nature Genetics, 45(12), 1428-1430. https://doi.org/10.1038/ng.2800

Solomon, D. A., Kim, T., Diaz-Martinez, L. A., Fair, J., Elkahloun, A. G., Harris, B. T., ... Waldman, T. (2011). Mutational inactivation of STAG2 causes aneuploidy in human cancer. Science, 333(6045), 1039-1043. https://doi.org/10.1126/science.1203619

Sotillo, R., Hernando, E., Díaz-Rodríguez, E., Teruya-Feldstein, J., Cordón-Cardo, C., Lowe, S. W., \& Benezra, R. (2007). Mad2 Overexpression Promotes Aneuploidy and Tumorigenesis in Mice. Cancer Cell, 11(1), 9-23. https://doi.org/10.1016/j.ccr.2006.10.019

Stam, S., Alberts, J., Gardel, M. L., \& Munro, E. (2015). Isoforms confer characteristic force generation and mechanosensation by myosin II filaments. Biophysical Journal, 108(8), 1997-2006. https://doi.org/10.1016/j.bpj.2015.03.030

Stepanova, T., Slemmer, J., Hoogenraad, C. C., Lansbergen, G., Dortland, B., De Zeeuw, C. I., ... Galjart, N. (2003). Visualization of Microtubule Growth in Cultured Neurons via the Use of EB3-GFP (End-Binding Protein 3-Green Fluorescent Protein). The Journal of Neuroscience, 23(7), 1655-1664.

Stewart, M. P., Helenius, J., Toyoda, Y., Ramanathan, S. P., Muller, D. J., \& Hyman, A. A. (2011). Hydrostatic pressure and the actomyosin cortex drive mitotic cell rounding. Nature, 469(7329), 226-231. https://doi.org/10.1038/nature09642

Stumpff, J., von Dassow, G., Wagenbach, M., Asbury, C., \& Wordeman, L. (2008). The Kinesin8 Motor Kif18A Suppresses Kinetochore Movements to Control Mitotic Chromosome Alignment. Developmental Cell, 14(2), 252-262.

https://doi.org/10.1016/j.devcel.2007.11.014

Stumpff, J., Wagenbach, M., Franck, A., Asbury, C. L., \& Wordeman, L. (2012). Kif18A and Chromokinesins Confine Centromere Movements via Microtubule Growth Suppression and Spatial Control of Kinetochore Tension. Developmental Cell, 22(5), 1017-1029. 
https://doi.org/10.1016/j.devcel.2012.02.013

Tanenbaum, M. E., \& Medema, R. H. (2010). Mechanisms of Centrosome Separation and Bipolar Spindle Assembly. Developmental Cell, 19(6), 797-806.

https://doi.org/10.1016/j.devcel.2010.11.011

Thawani, A., Kadzik, R. S., \& Petry, S. (2018). XMAP215 is a microtubule nucleation factor that functions synergistically with the y-tubulin ring complex. Nature Cell Biology, 20(5), 575585. https://doi.org/10.1038/s41556-018-0091-6

Théry, M., Jiménez-Dalmaroni, A., Racine, V., Bornens, M., \& Jülicher, F. (2007). Experimental and theoretical study of mitotic spindle orientation. Nature, 447(7143), 493-496. https://doi.org/10.1038/nature05786

Théry, M., Racine, V., Pépin, A., Piel, M., Chen, Y., Sibarita, J. B., \& Bornens, M. (2005). The extracellular matrix guides the orientation of the cell division axis. Nature Cell Biology, 7(10), 947-953. https://doi.org/10.1038/ncb1307

Thompson, S. L., Bakhoum, S. F., \& Compton, D. A. (2010). Mechanisms of Chromosomal Instability. Current Biology, 20(6), R285-R295. https://doi.org/10.1016/j.cub.2010.01.034

Thompson, S. L., \& Compton, D. A. (2008). Examining the link between chromosomal instability and aneuploidy in human cells. Journal of Cell Biology, 180(4), 665-672. https://doi.org/10.1083/jcb.200712029

Tighe, A., Johnson, V. L., Albertella, M., \& Taylor, S. S. (2001). Aneuploid colon cancer cells have a robust spindle checkpoint. EMBO Reports, 2(7), 609-614. https://doi.org/10.1093/embo-reports/kve127

Tomasek, J. J., \& Hay, E. D. (1984). Analysis of the role of microfilaments and microtubules in acquisition of bipolarity and elongation of fibroblasts in hydrated collagen gels. Journal of Cell Biology, 99(2), 536-549. https://doi.org/10.1083/jcb.99.2.536

Toyoshima, F., \& Nishida, E. (2007). Integrin-mediated adhesion orients the spindle parallel to the substratum in an EB1- and myosin X-dependent manner. EMBO Journal, 26(6), 14871498. https://doi.org/10.1038/sj.emboj.7601599

Urbani, L., \& Stearns, T. (1999). The centrosome. Current Biology, 9(9), R315-R317. 
Van Haren, J., Boudeau, J., Schmidt, S., Basu, S., Liu, Z., Lammers, D., ... Galjart, N. (2014). Dynamic microtubules catalyze formation of navigator-TRIO complexes to regulate neurite extension. Current Biology, 24(15), 1778-1785. https://doi.org/10.1016/j.cub.2014.06.037

Van Heesbeen, R. G. H. P., Raaijmakers, J. A., Tanenbaum, M. E., \& Medema, R. H. (2013). Nuclear envelope-associated dynein cooperates with Eg5 to drive prophase centrosome separation. Communicative \& Integrative Biology, 6(3), e23841.

https://doi.org/10.4161/cib.23841

Van Rijssel, J., \& Van Buul, J. D. (2012). The many faces of the guanine-nucleotide exchange factor trio. Cell Adhesion and Migration, 6(6), 482-487. https://doi.org/10.4161/cam.21418

Van Troys, M., Huyck, L., Leyman, S., Dhaese, S., Vandekerkhove, J., \& Ampe, C. (2008). Ins and outs of ADF/cofilin activity and regulation. European Journal of Cell Biology, 87(8-9), 649-667. https://doi.org/10.1016/j.ejcb.2008.04.001

Vicente, J. J., \& Wordeman, L. (2015). Mitosis, microtubule dynamics and the evolution of kinesins. Experimental Cell Research, 334(1), 61-69.

https://doi.org/10.1016/j.yexcr.2015.02.010

Vilar, E., \& Gruber, S. B. (2010). Microsatellite instability in colorectal cancerthe stable evidence. Nature Reviews Clinical Oncology, 7(3), 153-162.

https://doi.org/10.1038/nrclinonc.2009.237

Vodicska, B., Cerikan, B., Schiebel, E., \& Hoffmann, I. (2018). MISP regulates the IQGAP1/Cdc42 complex to collectively orchestrate spindle orientation and mitotic progression. Scientific Reports, 8(1), 1-12. https://doi.org/10.1038/s41598-018-24682-8

Walczak, C. E., Cai, S., \& Khodjakov, A. (2010). Mechanisms of chromosome behaviour during mitosis. Nature Reviews Molecular Cell Biology, 11(2), 91-102. https://doi.org/10.1038/nrm2832

Wallar, B. J., Stropich, B. N., Schoenherr, J. A., Holman, H. A., Kitchen, S. M., \& Alberts, A. S. (2006). The basic region of the diaphanous-autoregulatory domain (DAD) is required for autoregulatory interactions with the diaphanous-related formin inhibitory domain. Journal of Biological Chemistry, 281(7), 4300-4307. https://doi.org/10.1074/jbc.M510277200

Wandke, C., Barisic, M., Sigl, R., Rauch, V., Wolf, F., Amaro, A. C., ... Geley, S. (2012). Human 
chromokinesins promote chromosome congression and spindle microtubule dynamics during mitosis. Journal of Cell Biology, 198(5), 847-863.

https://doi.org/10.1083/jcb.201110060

Watanabe, N., Kato, T., Fujita, A., Ishizaki, T., \& Narumiya, S. (1999). Cooperation between mDia1 and ROCK in Rho-induced actin reorganization. Nature Cell Biology, 1(3), 136-143. https://doi.org/10.1038/11056

Watanabe, N., Madaule, P., Reid, T., Ishizaki, T., Watanabe, G., Kakizuka, A., ... Narumiya, S. (1997). p140mDia, a mammalian homolog of Drosophila diaphanous, is a target protein for Rho small GTPase and is a ligand for profilin. EMBO Journal, 16(11), 3044-3056. https://doi.org/10.1093/emboj/16.11.3044

Watanabe, T., Wang, S., Noritake, J., Sato, K., Fukata, M., Takefuji, M., ... Kaibuchi, K. (2004). Interaction with IQGAP1 links APC to Rac1, Cdc42, and actin filaments during cell polarization and migration. Developmental Cell, 7(6), 871-883. https://doi.org/10.1016/j.devcel.2004.10.017

Waterman-Storer, C. M., Worthylake, R. A., Liu, B. P., Burridget, K., \& Salmon, E. D. (1999). Microtubule growth activates Rac1 to promote lamellipodial protrusion in fibroblasts. Nature Cell Biology, 1(1), 45-50. https://doi.org/10.1038/9018

Weaver, B. A. A., Silk, A. D., Montagna, C., Verdier-Pinard, P., \& Cleveland, D. W. (2007). Aneuploidy Acts Both Oncogenically and as a Tumor Suppressor. Cancer Cell. https://doi.org/10.1016/j.ccr.2006.12.003

Weber, K. L., Sokac, A. M., Berg, J. S., Cheney, R. E., \& Bement, W. M. (2004). A microtubulebinding myosin required for nuclear anchoring and spindle assembly. Nature, 431(7006), 325-329. https://doi.org/10.1038/nature02834

Wen, Y., Eng, C. H., Schmoranzer, J., Cabrera-Poch, N., Morris, E. J. S., Chen, M., ... Gundersen, G. G. (2004). EB1 and APC bind to mDia to stabilize microtubules downstream of Rho and promote cell migration. Nature Cell Biology, 6(9), 820-830. https://doi.org/10.1038/ncb1160

Wendt, T., Taylor, D., Trybus, K. M., \& Taylor, K. (2001). Three-dimensional image reconstruction of dephosphorylated smooth muscle heavy meromyosin reveals asymmetry 
in the interaction between myosin heads and placement of subfragment 2. PNAS, 98(8), 4361-4366.

Werner, M., Munro, E., \& Glotzer, M. (2007). Astral Signals Spatially Bias Cortical Myosin Recruitment to Break Symmetry and Promote Cytokinesis. Current Biology, 17(15), 12861297. https://doi.org/10.1016/j.cub.2007.06.070

Wertheimer, E., Gutierrez-Uzquiza, A., Rosemblit, C., Lopez-Haber, C., Sosa, M. S., \& Kazanietz, M. G. (2012). Rac signaling in breast cancer: A tale of GEFs and GAPs. Cellular Signalling, 24(2), 353-362. https://doi.org/10.1016/j.cellsig.2011.08.011

Whalley, H. J., Porter, A. P., Diamantopoulou, Z., White, G. R. M., Castañeda-Saucedo, E., \& Malliri, A. (2015a). Cdk1 phosphorylates the Rac activator Tiam1 to activate centrosomal Pak and promote mitotic spindle formation. Nature Communications, 6(1), 7437. https://doi.org/10.1038/ncomms8437

Whalley, H. J., Porter, A. P., Diamantopoulou, Z., White, G. R. M., Castañeda-Saucedo, E., \& Malliri, A. (2015b). Cdk1 phosphorylates the Rac activator Tiam1 to activate centrosomal Pak and promote mitotic spindle formation. Nature Communications, 6(June). https://doi.org/10.1038/ncomms8437

Willard, F. S., Kimple, R. J., \& Siderovski, D. P. (2004). Return of the GDI: The GoLoco Motif in Cell Division. Annual Review of Biochemistry, 73(1), 925-951. https://doi.org/10.1146/annurev.biochem.73.011303.073756

Winterhoff, M., \& Faix, J. (2015). Actin-filament disassembly: It takes two to shrink them fast. Current Biology, 25(11), R450-R452. https://doi.org/10.1016/j.cub.2015.04.050

Wittmann, T., Hyman, A., \& Desai, A. (2001). The spindle: A dynamic assembly of microtubules and motors. Nature Cell Biology, 3(1), E28-E34. https://doi.org/10.1038/35050669

Wojnacki, J., Quassollo, G., Marzolo, M. P., \& Cáceres, A. (2014). Rho GTPases at the crossroad of signaling networks in mammals. Small GTPases, 5(1), e28430. https://doi.org/10.4161/sgtp.28430

Wood, L. D., Parsons, D. W., Jones, S., Lin, J., Sjöblom, T., Leary, R. J., ... Vogelstein, B. (2007). The genomic landscapes of human breast and colorectal cancers. Science, 318(5853), 1108-1113. https://doi.org/10.1126/science.1145720 
Woodard, G. E., Huang, N.-N., Cho, H., Miki, T., Tall, G. G., \& Kehrl, J. H. (2010). Ric-8A and Gi Recruit LGN, NuMA, and Dynein to the Cell Cortex To Help Orient the Mitotic Spindle. Molecular and Cellular Biology, 30(14), 3519-3530. https://doi.org/10.1128/mcb.00394-10

Woodcock, S. A., Rushton, H. J., Castañeda-Saucedo, E., Myant, K., White, G. R. M., Blyth, K., ... Malliri, A. (2010). Tiam1-Rac Signaling Counteracts Eg5 during Bipolar Spindle Assembly to Facilitate Chromosome Congression. Current Biology, 20(7), 669-675. https://doi.org/10.1016/j.cub.2010.02.033

Worthylake, R. A., Lemoine, S., Watson, J. M., \& Burridge, K. (2001). RhoA is required for monocyte tail retraction during transendothelial migration. Journal of Cell Biology, 154(1), 147-160. https://doi.org/10.1083/jcb.200103048

Yajima, J., Edamatsu, M., Watai-Nishii, J., Tokai-Nishizumi, N., Yamamoto, T., \& Toyoshima, Y. Y. (2003). The human chromokinesin Kid is a plus end-directed microtubule-based motor. EMBO Journal, 22(5), 1067-1074. https://doi.org/10.1093/emboj/cdg102

Yamaguchi, H., \& Condeelis, J. (2007). Regulation of the actin cytoskeleton in cancer cell migration and invasion. Biochimica et Biophysica Acta - Molecular Cell Research, 1773(5), 642-652. https://doi.org/10.1016/j.bbamcr.2006.07.001

Yang, Z., Tulu, U. S., Wadsworth, P., \& Rieder, C. L. (2007). Kinetochore Dynein Is Required for Chromosome Motion and Congression Independent of the Spindle Checkpoint. Current Biology, 17(11), 973-980. https://doi.org/10.1016/j.cub.2007.04.056

Yao, G. (2014). Modelling mammalian cellular quiescence. Interface Focus, 4(20130074). https://doi.org/10.1098/rsfs.2013.0074

Yardimci, H., Van Duffelen, M., Mao, Y., Rosenfeld, S. S., \& Selvin, P. R. (2008). The mitotic kinesin CENP-E is a processive transport motor. Proceedings of the National Academy of Sciences of the United States of America, 105(16), 6016-6021. https://doi.org/10.1073/pnas.0711314105

Yoshida, T., Zhang, Y., Rivera Rosado, L. A., Chen, J., Khan, T., Moon, S. Y., \& Zhang, B. (2010). Blockade of Rac1 activity induces G1 cell cycle arrest or apoptosis in breast cancer cells through downregulation of cyclin D1, survivin, and X-linked inhibitor of apoptosis protein. Molecular Cancer Therapeutics, 9(6), 1657-1668. https://doi.org/10.1158/1535- 
7163.MCT-09-0906

Yost, S., De Wolf, B., Hanks, S., Zachariou, A., Marcozzi, C., Clarke, M., ... Rahman, N. (2017). Biallelic TRIP13 mutations predispose to Wilms tumor and chromosome missegregation. Nature Genetics, 49(7), 1148-1151. https://doi.org/10.1038/ng.3883

Zasadil, L. M., Britigan, E. M. C., Ryan, S. D., Kaur, C., Guckenberger, D. J., Beebe, D. J., ... Weaver, B. A. (2016). High rates of chromosome missegregation suppress tumor progression but do not inhibit tumor initiation. Molecular Biology of the Cell, 27(13), 19811989. https://doi.org/10.1091/mbc.e15-10-0747

Zhao, Z. S., Lim, J. P., Ng, Y. W., Lim, L., \& Manser, E. (2005). The GIT-associated kinase PAK targets to the centrosome and regulates Aurora-A. Molecular Cell, 20(2), 237-249. https://doi.org/10.1016/j.molcel.2005.08.035

Zheng, H., Zheng, Y., Li, X., Takahashi, H., Hara, T., Masuda, S., ... Takano, Y. (2008). Arp2/3 Overexpression Contributed to Pathogenesis, Growth and Invasion of Gastric Carcinoma. Anticancer Research, 28, 2225-2232.

Zheng, M., Simon, R., Mirlacher, M., Maurer, R., Gasser, T., Forster, T., ... Schraml, P. (2004). TRIO Amplification and Abundant mRNA Expression Is Associated with Invasive Tumor Growth and Rapid Tumor Cell Proliferation in Urinary Bladder Cancer. The American Journal of Pathology, 165(1), 63-69. https://doi.org/10.1016/S0002-9440(10)63275-0

Zheng, Y. (2001). Dbl family guanine nucleotide exchange factors. Trends in Biochemical Sciences, 26(12), 724-732. https://doi.org/10.1016/S0968-0004(01)01973-9

Zhou, B. S., \& Elledge, S. J. (2000). Checkpoints in Perspective. Nature, 408(6811), 433-439.

Zhu, C., Zhao, J., Bibikova, M., Leverson, J. D., Bossy-Wetzel, E., Fan, J.-B., ... Jiang, W. (2005). Functional Analysis of Human Microtubule-based Motor Proteins, the Kinesins and Dyneins, in Mitosis/Cytokinesis Using RNA Interference. Molecular Biology of the Cell, 16(7), 3187-3199. https://doi.org/10.1091/mbc.E05

Zhu, M., Settele, F., Kotak, S., Luis, S. P., Ehret, L., Ponting, C. P., .. Hoffmann, I. (2013). MISP is a novel Plk1 substrate required for proper spindle orientation and mitotic progression. Journal of Cell Biology, 200(6), 773-787. https://doi.org/10.1083/jcb.201207050 


\section{References}

Zhu, X. L., Liang, L., \& Ding, Y. Q. (2008). Overexpression of FMNL2 is closely related to metastasis of colorectal cancer. International Journal of Colorectal Disease, 23(11), 10411047. https://doi.org/10.1007/s00384-008-0520-2

Zigmond, S. H., Evangelists, M., Boone, C., Yang, C., Dar, A. C., Sicheri, F., ... Pring, M. (2003). Formin Leaky Cap Allows Elongation in the Presence of Tight Capping Proteins. Current Biology, 13(20), 1820-1823. https://doi.org/10.1016/j.cub.2003.09.057 


\section{Acknowledgements - Danksagung}

An dieser Stelle möchte ich mich bei allen bedanken, die mir es ermöglicht haben diesen Weg zu gehen sowie zum Gelingen dieser Arbeit beigetragen und mich unterstützt haben.

Zunächst gilt mein Dank meinem Doktorvater Prof. Dr. Holger Bastians, der mir die Möglichkeit gegeben hat an diesem spannenden Thema zu arbeiten. Danke für die stetige Betreuung und die Bereitschaft jederzeit Fragen zu beantworten, zu diskutieren und die daraus entstehenden Ideen, die das Projekt vorangetrieben haben.

Außerdem bedanke ich mich hiermit bei den Mitgliedern meines Thesis Committee Prof. Dr. Jörg Großhans und Prof. Dr. Michael Zeisberg, die mich während der letzten Jahre gut bereut und beraten haben. Zudem gilt mein Dank Prof. Dr. Heike Krebber für die Möglichkeit Laborräume und Equipment nutzen zu können.

Ebenfalls bedanken möchte ich mich bei Prof. Dr. Andreas Janshoff und Dr. Bastian Rouven Brückner für die sehr aufschlussreiche, spannende und abwechslungsreiche Kollaboration. Danke Basti für die Auswertung der Daten und vor allem für die vielen unterhaltsamen Momente am AFM.

Ein ganz besonderer Dank gilt allen Mitgliedern aus der Arbeitsgruppe, die für eine wunderbare Arbeitsatmosphäre verantwortlich waren und immer mit Rat und Tat zur Seite standen. Vor allem möchte ich mich bei Yu-Chi für das Lesen von Teilen dieser Arbeit bedanken und bei Lena für das Generieren der Klone sowie der Unterstützung bei kniffligen Western Blots. Außerdem bedanke ich mich bei Nico und Karo für die lustigen Mittagspausen in der Mensa, die immer eine nette Abwechslung vom Laboralltag waren. Herzlichen bedanken möchte ich mich noch bei Elina, Karo und Ann-Kathrin für die hilfreichen, fachlichen Diskussion und vor allem für die vielen lustigen gemeinsamen Stunden sowohl im Labor als auch abseits der Arbeit.

Schließlich gilt mein größter Dank meinen Eltern, Susanne und Frank sowie meinem Freund Jakob für die immerwährende Unterstützung, Rückhalt und Verständnis sowie Liebe. Ohne euch wäre diese Arbeit nicht möglich gewesen und ich danke euch von ganzen Herzen. 